\title{
Tuning Mesoporous Silica Structures via RAFT Polymers: From Multiblock Copolymers as new Templates to Surface Modification
}

\author{
Dissertation \\ zur Erlangung des mathematisch-naturwissenschaftlichen Doktorgrades \\ "Doctor rerum naturalium" \\ der Georg-August-Universität Göttingen \\ im Promotionsprogramm Chemie \\ der Georg-August University School of Science \\ (GAUSS) \\ vorgelegt von \\ Sonja Schmidt \\ aus Kassel
}

Göttingen, 2018 
Betreuungsausschuss

Prof. Dr. Philipp Vana, MBA

Institut für Physikalische Chemie Georg-August-Universität Göttingen

Prof. Dr. Marcus Müller

Institut für Theoretische Physik

Georg-August-Universität Göttingen

\section{Mitglieder der Prüfungskommission}

\section{Referent}

Prof. Dr. Philipp Vana, MBA

Institut für Physikalische Chemie Georg-August-Universität Göttingen

\section{Korreferent}

Prof. Dr. Marcus Müller

Institut für Theoretische Physik Georg-August-Universität Göttingen

\section{Weitere Mitglieder der Prüfungskommission}

Prof. Dr. Burkhard Geil

Prof. Dr. Michael Buback

Prof. Dr. Ricardo Mata

Dr. Florian Ehlers

Tag der mündlichen Prüfung:
Institut für Physikalische Chemie Georg-August-Universität Göttingen

Institut für Physikalische Chemie Georg-August-Universität Göttingen

Institut für Physikalische Chemie Georg-August-Universität Göttingen

Institut für Physikalische Chemie Georg-August-Universität Göttingen

09.02.2018 


\section{Acknowledgements}

First of all, I want to thank my supervisor Prof. Dr. Philipp Vana for his guidance and support during the past four years. I am thankful for this exciting topic and all the inspiring discussions. Furthermore, I appreciate that he gave me the opportunity to take part in an international project and to do a research period abroad during the time of my thesis.

I would also like to thank my co-supervisor Prof. Dr. Marcus Müller for his kind support, interesting discussions and his perspectives of the theoretical point of view.

Prof. Dr. Buback, Prof. Dr. Burkhard Geil, Prof. Dr. Ricardo Mata and Dr. Florian Ehlers are gratefully acknowledged for being part of my thesis committee.

I would like to thank Prof. Galo J. A. A. Soler-Illia for letting me visit his working group at Universidad Nacional de General San Martín, Buenos Aires. I also want to thank Prof. Omar Azzaroni for giving me the possibility to use the facilities of his laboratory. I am grateful for meeting all the incredible and lovely people during my stay. Especially, I would like to thank Dr. Cintia Conteras for showing me around, helping me through the scientific challenges and the daily struggle caused by my lack of Spanish. I also want to express my gratitude to Sebastián Alberti for our fruitful collaboration even beyond continents away. I also like to thank Dr. Qiyun Tang who is also part of this international cooperation to help me with theoretical simulations.

I want to thank Volker Radisch and Matthias Hahn for the introduction to the measurements of scanning electron microscopy and providing a fundamental knowledge.

I would like to thank all the group members of the MMC and TuMChem groups for the friendly working environment, interesting discussions and enjoyable time during my thesis. My special thanks go to my office and laboratory colleagues Martin Kassel, Torsten Fornefeld, Niklas Frerichs, Dr. Nicolai Sörensen, Dr. Sebastian Smolne, Dr. Dennis Hübner, Dr. Julia 
Schütz and Annika Nitschke. Here, I also would like Dr. Hans-Peter Vögele for the all supporting discussions. Additionally, I want to thank my bachelor student Dominik Ruhr for his contributions to this work.

I sincerely want to thank Katharina Dabow, Dr. Michael Hendrich, Byron Staudt, Annika Nitschke, Niklas Frerichs, Dr. Dennis Hübner, Alex Groschopp and Judith Steinhoff for carefully proofreading this work. Especially, I want to thank Laura Dzemski and Johnny Lim for proofreading this thesis with regard to English grammar and style.

I want to thank all my friends. Thank you for your support and for giving me the right distractions from my scientific daily routine.

Finally, I would like to express my gratitude to my family for their support through the times of my studies and thesis. Without you, I would not be where I am now. I also want to thank Martin Dzemski for all his support and making me smile in every possible situation. 


\section{Abstract}

In this work, new procedures to tailor mesoporous silica thin films are presented. On the one hand, linear multiblock copolymers were utilized as structure directing agents for the synthesis of mesoporous silica thin films. On the other hand, the design of an interfacial architecture using the surface-initiated reversible addition-fragmentation chain transfer (SI-RAFT) polymerization was accomplished which altered the transport properties of the mesoporous silica thin films.

Linear multiblock copolymer systems were synthesized using polyfunctional RAFT agents. The dependence of their microphase separation behavior on the annealing technique was systematically studied using atomic force microscopy (AFM). Multiblock copolymers containing polystyrene (PS) and poly(n-butyl acrylate) (PBA) segments showed cylindrical morphologies using thermal annealing. Thin films of amphiphilic multiblock copolymers consisting of PS and poly( $N$-isopropylacrylamide) (PNIPAM) segments were annealed using selective solvents. Perpendicular PS cylinders arranged in a PNIPAM matrix were found for a volume fraction $0.59<f_{\text {PNIPAM }}<0.77$ using selective solvent mixtures of methanol-toluene or methanol-tetrahydrofuran. The formation of this morphology was independent of the segment number. Different morphologies than those observed using solvent mixtures were achieved using only one type of solvent. Furthermore, the formation of the self-assembled structure was less pronounced when increasing the film thickness. Additionally, it was found that the morphology of triblock copolymers using solvent annealing was inverted via subsequent thermal treatment. The observed inversion was caused by the stronger temperaturedependency of the surface energy of the PS segments.

For the first time, these amphiphilic multiblock copolymers were used as structure directing agents in the formation of mesoporous silica thin films. A general process was developed using evaporation-induced selfassembly. The combination of solvent annealing upon film deposition and 
oxygen plasma treatment to remove the organic template was found to be an effective way to form mesoporous silica thin films. Scanning electron microscopy and AFM were used to visualize the porous structure of the films after template removal. The optimal sol aging time was found to be three days. Longer aging times led to a disappearance of the porous structure caused by condensation of the silicate species. Moreover, an effect of the amount of multiblock copolymers in solution on the organization of the film was observed. Higher amounts of multiblock copolymers promoted the self-assembly of the organic-inorganic interface and led to more pronounced organization. In addition, it was observed that the pore size depends on the number of segments in the multiblock copolymer. Multiblock copolymers with segment numbers greater than three arrange into a linear string of molecular micelles. Therefore, pore sizes smaller than $16 \mathrm{~nm}$ are accessible. Single flower-like intramolecular micelles are formed for multiblock copolymers containing less than four segments. Here, pore sizes larger than $16 \mathrm{~nm}$ are observed. To completely characterize the films ellipsometry and nitrogen adsorption-desorption measurements were performed. Ellipsometry revealed thicknesses below $40 \mathrm{~nm}$ and nitrogen adsorption-desorption isotherms indicated slit-shaped or particle-like interconnected pores.

To exploit the influence of surface tethered polymer brushes on the transport properties of mesoporous silica thin film, a hybrid material containing the thermoresponsive PNIPAM was synthesized. Here, mesoporous silica thin films were modified with RAFT agent carrying silica anchor groups. These functionalized mesoporous materials were subsequently used in SI-RAFT of PNIPAM and systematically characterized. Ellipsometry measurements revealed the selective modification of the outer sphere of the mesoporous silica thin film. Furthermore, cyclic voltammetry measurements using redox probes were performed to give a better understanding of the temperature-induced switching ability of PNIPAM as a gating system. The results revealed that cationic probes could be shuttled across or be excluded depending on the temperature. Anionic redox probes were expelled independent of the temperature. The functionalization was proven to be successful and leads to thermosensitive cation-selective mesochannels. The unique transport properties of the hybrid interface could be observed due to the presence of the cooperative interaction between the gating of the PNIPAM layer and the anion entry exclusion exerted by the mesoporous silica surface. 


\section{Table of Content}

1 Introduction and Motivation ....................................................... 1

2 Microphase Separation of Multiblock Copolymers .................. 5

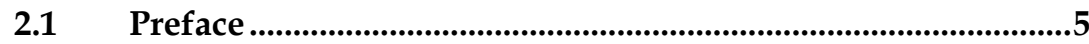

2.2 Fundamentals of RAFT Polymerization .....................................6

2.2.1 The RAFT Mechanism..........................................................6

2.2.2 Linear $(\mathrm{AB})_{\mathrm{n}}$-Multiblock Copolymers via Polyfunctional RAFT Agents ........................................................................ 9

2.3 Fundamentals of Microphase Separation of Block

Copolymers ..................................................................................12

2.4 Design of Multiblock Copolymers via Polyfunctional RAFT

Agent ..................................................................................................17

2.4.1 Synthesis of Multiblock Polystyrene ...................................20

2.4.2 Synthesis of Multiblock Copolymers .................................23

2.5 Investigation of the Microphase Separation of Multiblock

Copolymer Systems via AFM ..................................................30

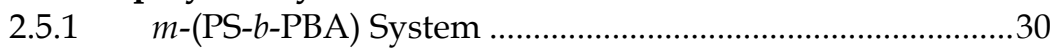

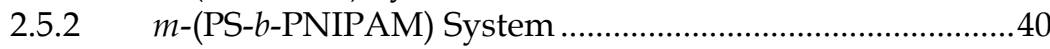

2.6 Conclusions.....................................................................................69

3 Novel Mesoporous Silica Thin Films via Multiblock

Copolymers ............................................................................. 70

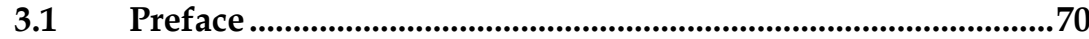

3.2 Classification of porous materials ............................................71

3.3 Fundamentals of Mesostructured Silica Thin Films ..............73

3.4 Synthesis of Mesoporous Silica Thin Films by Multiblock

Copolymers ...........................................................................................76

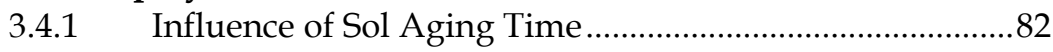

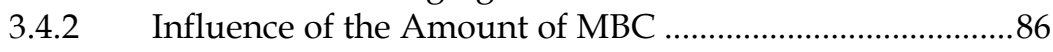


3.4.3 Influence of the Chemical Nature of the MBC upon Mesostructure Formation.

Conclusions.......................................................................94

4 Thermosensitive Cation-Selective Mesochannels ................. 95

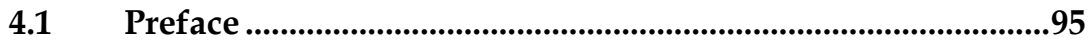

4.2 Fundamentals of RAFT Polymerization at Surfaces..............97

4.3 Synthesis of Thermo-Responsive Mesoporous Silica Thin

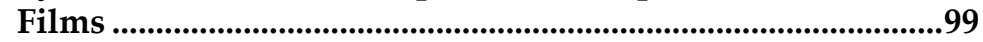

4.4 Cation-Selective Behavior of the Thermosensitive Mesoporous Silica Thin Film..................................................105

4.5 Conclusion..........................................................................110

5 Closing Remarks and Future Perspectives ......................... 112

6 Experimental Section ........................................................ 116

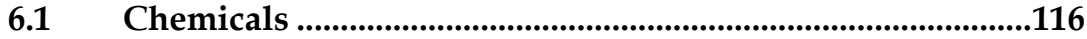

6.2 Analytical Methods...............................................................116

6.2.1 Atomic Force Microscopy …..............................................116

6.2.2 Size-Exclusion-Chromatography ....................................117

6.2.3 Mass Spectroscopy ...........................................................117

6.2.4 Nuclear Magnetic Resonance Spectroscopy ......................118

6.2.5 Scanning Electron Microscopy ..........................................118

6.2.6 Thermogravimetric Analysis............................................118

6.2.7 Water Contact Angle ..........................................................118

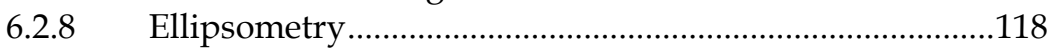

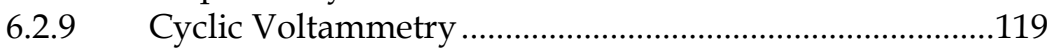

6.2.10 Dynamic Light Scattering .................................................119

6.2.11 Differential Scanning Calorimetry ….................................120

6.2.12 X-ray Photoelectron Spectroscopy .....................................120

6.2.13 Small-Angle X-ray Scattering ............................................120

6.2.14 Nitrogen Adsorption-Desorption Isotherms.....................120

6.3 Syntheses ...........................................................................121

6.3.1 Syntheses of RAFT Agents ..............................................121

6.4 Synthesis of Mesoporous Silica Thin Films ............................123

6.4.1 Mesoporous Silica Thin Films with Block Copolymer....123

6.4.2 Mesoporous Silica Thin Films via Multiblock Copolymers 124

6.5 Functionalization of Surfaces ................................................125

6.5.1 Immobilization of RAFT Agent onto a Mesoporous Silica

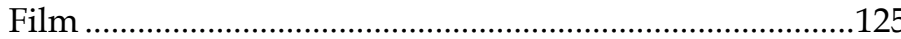

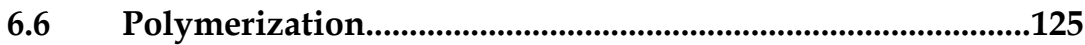


6.6.1 Homopolymerization of Styrene with polyRAFT. .125

6.6.2 Block Copolymerization of Styrene Homopolymer with n-Butyl Acrylate ...............................................................126

6.6.3 Block Copolymerization of Styrene Homopolymer with N-Isopropylacrylamide ..................................................126

6.6.4 Cleavage of RAFT Groups ..............................................127

6.6.5 Surface-initiated RAFT Polymerization of N-Isopropylacrylamide ....................................................127

6.7 Microphase Separation of Multiblock Copolymers ..............128

6.7.1 $m$-(PS- $b$-PBA) ….......................................................128

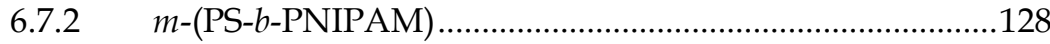

Appendices .................................................................................... 129

Abbreviations ............................................................................ 133

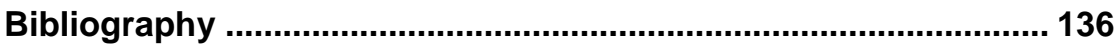





\section{Introduction and Motivation}

From the beginning of mankind until modern times the development of humans is related to materials which are available in nature. ${ }^{[1,2]}$ In the last century, scientists and engineers have developed methods and procedures to create highly functional materials which are inspired by nature. Zeolite is one of the naturally occurring materials which have been mimicked by scientists since it was discovered by the Swedish mineralogist Cronstedt in 1756. He observed that the material produced large amounts of steam during rapid heating. Therefore, Cronstedt called the mineral a "zeolite" which originates from the Greek words zeo - to boil and lithos - a stone. Zeolites are crystalline alumosilicates which exhibit cavities and channels of defined shape and size. Only a hundred years after the discovery, the first synthetic zeolites were prepared. ${ }^{[3]}$ Depending on the type of zeolite, the pore size ranges from $0.4-1.5 \mathrm{~nm}$. Nowadays over 200 different types of zeolites are synthetically accessible. Small molecules like water can be adsorbed and ions reversibly exchanged due to the small pore size and large surface area.[4]

In general, porous materials can be divided by their pore size. If their size is less than $2 \mathrm{~nm}$, the pores are microporous. Mesostructured materials exhibit pore sizes from 2 to $50 \mathrm{~nm}$. Pores larger than $50 \mathrm{~nm}$ are classified as macroporous. During the last decades new synthetic methods were developed to create such mesoporous materials. The field of applications was widening since the pore space was not restricted to small molecules. Mesostructured pores can act as nanoreactors, catalysts or gating-systems. One well-established procedure to prepare mesoporous silica materials is the sol-gel process. In this procedure metal oxides are transformed into a framework by hydrolysis followed by condensation.

By the introduction of the Mobil's group procedure, a new class of mesoporous silica materials was created. ${ }^{[5-7]}$ It is based on the combination of sol-gel chemistry and supramolecular templates. Especially, the formation of mesoporous silica materials as thin films introduces the possibility to create new systems. Mesoporous silica thin films combine properties such as controlled pore size, shape, symmetry with optical surface properties and substrate support. ${ }^{[8]}$ Moreover, mesoporous silica thin films exhibit tailorable pore size and interconnectivity. In addition, they are robust and offer enhanced accessibility to molecules. These 
properties have made mesoporous thin films useful for potential applications in catalysis, sensors, or energy-conversion systems. ${ }^{[9-12]}$ The incorporation of organic functionalities within the pores opened up the pathway to hybrid interfacial architectures. These organic-inorganic structures led to selective porous materials which are of particular interest in sensing, separation applications or intelligent drug delivery devices. ${ }^{[13,14]}$

The design of mesostructured silica thin films can be tailored by their organic templates. Among all template classes, polymers are recognized as the most unique group. Polymers can be tailored by adjusting molar mass, composition, monomer choice and architecture. In particular, block copolymers have drawn great attention due to their unique architecture. Block copolymers consist of two or more homopolymer block units which are covalently bound to each other. Besides diblock copolymers, triblock or linear multiblock polymers also exist. Those block copolymers show the ability to self-assemble into microstructures which results from the immiscibility of the distinct blocks. Different morphologies such as lamellae or cylinders are accessible depending on the comonomer composition. These macromolecular architectures can be designed by synthesis strategies such as reversible-deactivation radical polymerization (RDRP). RDRP enables the polymerization of commercially available monomers with the use of special controlling agents. One of the most versatile polymerization techniques is reversible addition-fragmentation chain transfer (RAFT). The mechanism of RAFT polymerization is based on a degenerative chaintransfer, in which an equilibrium between propagating radical species and dormant chains is present. Besides common RAFT agents, polyfunctional RAFT agents are accessible which contain multiple RAFT groups per molecule. Using these polyfunctional RAFT agents, multiblock copolymers can be prepared in only two polymerization steps. This approach is the most facile one compared to conventional methods. ${ }^{[15]}$ Due to their unique structure suitable applications from lithography to templates in mesostructured films are possible.

This work is meant as a contribution to the field of mesoporous silica thin films with distinct properties. Fundamental and versatile synthesis procedures to form mesoporous silica thin films are explored. In this thesis, two different approaches are pursued. For the development of mesoporous silica thin films the preparation process and properties of the precursors can be tailored. Here, new organic macromolecular templates are designed, namely multiblock copolymers are prepared using polyfunctional RAFT agents. Their microphase separation behavior is investigated in detail by varying conditions such as temperature, solvent and composition. The ability of multiblock copolymers to self-assemble into a variety of morphologies is exploited to control structure formation on the nanoscale. 
Therefore, inorganic silicate building blocks and multiblock copolymers are combined. Multiblock copolymers are used as sacrificial components to arrange the silicate species into hybrid polymer-inorganic mesophase materials. The removal of the polymer leads to mesoporous thin films with controlled shape and dimension. By using suitable multiblock copolymers as structure directing agents a universal and versatile technique is established to prepare mesoporous silica thin films. The second approach is to post-modify the mesoporous silica thin films to alter their properties. Consequently, mesoporous silica thin films are post-functionalized by surface-tethered thermosensitive polymers. The temperature-induced property changes of the films are systematically characterized. 



\section{Microphase Separation of Multiblock Copolymers}

\subsection{Preface}

Block copolymer thin films have gained great attention in different technological areas due to their ability to self-assemble into nanometersized domains. Besides the formation of highly ordered regions, the variation of size, shape and composition of the molecules provides qualification for a wide range of applications such as microelectronic devices, drug delivery, nanoporous materials or lithography.[16-19] Probably the most- and well-studied system is the diblock copolymer which is a macromolecule composed of two chemically different polymers covalently bound to each other. The appearance of self-assembled microphase structures results from the immiscibility of the distinct blocks depending on the comonomer composition into different morphologies such as lamellar or cylindrical. The thermodynamic driving force of the microphase separation minimizes the contact surface of those distinct blocks. It depends on the product of the Flory-Huggins parameter and the degree of polymerization.

In the last two decades there has been growing interest in the class of multiblock copolymers. A multiblock copolymer incorporates alternating homogenous segments of different monomers which are covalently bound. ${ }^{[15]}$ Experimental and theoretical studies on their phase behavior has been performed. [20-31] It has been demonstrated that linear $(A B)_{n}$-multiblock copolymers show similar phase behavior as AB diblock copolymers. Therefore, depending on the composition and the degree of incompatibility of the multiblock copolymer, ordered structures like lamellar, cylindrical, spherical or gyroid can be observed.[21,24,25,30] The synthesis approach to such synthetically demanding macromolecules is accessible by reversible deactivation radical polymerization (RDRP), such as atom transfer radical polymerization (ATRP), nitroxide-mediated radical polymerization (NMP) or reversible addition-fragmentation chain transfer (RAFT) polymerization. These techniques allow for the preparation of well-defined polymers with narrow molar mass distributions and chain-end functionality. However, the production of multiblock copolymers remains a synthetical challenge. The 
main issues are the decrease of chain-end fidelity with increasing block number and mandatory removal of any unreacted monomer before further block extension. ${ }^{[32-34]}$ You et al. and Motokochu and co-workers introduced a pathway which offers the possibility to synthesize $(A B)_{n}$-multiblock copolymers in only two polymerization steps. ${ }^{[35-38]}$ A new class of controlling agents, the polyfunctional RAFT agents, was employed in their studies to form well-defined multiblock architectures.

In this work multiblock copolymers based on three different monomers, styrene, $n$-butyl acrylate and $N$-isopropylacrylamide were prepared via reversible addition-fragmentation chain transfer (RAFT) polymerization using polyfunctional RAFT agents. The synthesized $(\mathrm{AB})_{\mathrm{n}}$-multiblock copolymers were characterized via nuclear magnetic resonance (NMR) spectroscopy, size-exclusion-chromatography (SEC) and differential scanning calorimetry (DSC). A detailed study of the microphase separation behavior of their thin films is given using atomic force microscopy (AFM).

\subsection{Fundamentals of RAFT Polymerization}

\subsubsection{The RAFT Mechanism}

Reversible addition-fragmentation chain transfer (RAFT) polymerization, established by CSIRO and Zard et al., is a versatile synthetic method to prepare highly functional polymers. ${ }^{[39-44]}$ This reversible deactivation radical polymerization technique is mediated by thiocarbonylthio compounds such as dithioesters, trithiocarbonates, xanthates or thiocarbamates. ${ }^{[40-42,45]}$ Figure 2.1 shows the general structure of a RAFT agent. It possesses a leaving group $\mathrm{R}$ and stabilizing Z-group.<smiles>[R]SC([Z])=S</smiles>

Figure 2.1. General structure of a RAFT agent, $\mathrm{Z}$ displays the stabilizing group and $\mathrm{R}$ the re-initiation leaving group.

The mechanism of RAFT is based on the principal of an additionfragmentation equilibrium shown in Scheme 2.1. Initiation and radicalradical termination are also present as in the conventional radical polymerization. The reaction is started by a free-radical source which can be for example a decomposing radical initiator (azo- or peroxide compound). The initiator decomposes into two fragments $\left(I^{*}\right)$, which react with a 
monomer molecule $(M)$ to form a propagating radical $\left(P_{n}{ }^{*}\right)$. In the next reaction step, the propagating radical reacts to the thiocarbonylthio compound (1) followed by the fragmentation of the intermediate radical (2) and results in a polymeric thiocarbonylthio compound (3) and a new radical $\left(R^{\circ}\right)$. This radical can react with further monomer molecules and form a new propagating radical $\left(\mathrm{P}_{\mathrm{m}}{ }^{*}\right)$. Due to the rapid equilibrium between the active propagating radicals $\left(\mathrm{P}_{\mathrm{n}}{ }^{*}\right.$ and $\left.\mathrm{P}_{\mathrm{m}}{ }^{*}\right)$ and the dormant polymeric thiocarbonylthio compound (3) via the intermediate (4) the probability for all chains to grow is equal. This leads to equal molar masses of the growing polymer chains and low dispersity. After completing (or stopping) the polymerization most of the chains which possess the thiocarbonylthio endgroup are dormant and can be used as macroRAFT agents in a following polymerization.[40-42] Furthermore, polymerizations can be performed in bulk, solution, emulsion or suspension. There are no particular limits on solvent or reaction temperature. Due to this fact a diversity of topologies and functionalities, such as star polymer, diblock, triblock or multiblock copolymers, are accessible.

In a typical RAFT polymerization, the RAFT agent is added in a specific amount to a solution of monomer and initiator (if necessary solvent). The choice of the RAFT agent depends on the monomer being polymerized. On the one hand, the intermediates (2) and (4) need to fragment rapidly without side reactions. On the other hand, the cleaved radical $\left(R^{*}\right)$ needs to be effective in reinitiating the polymerization (Scheme 2.1).

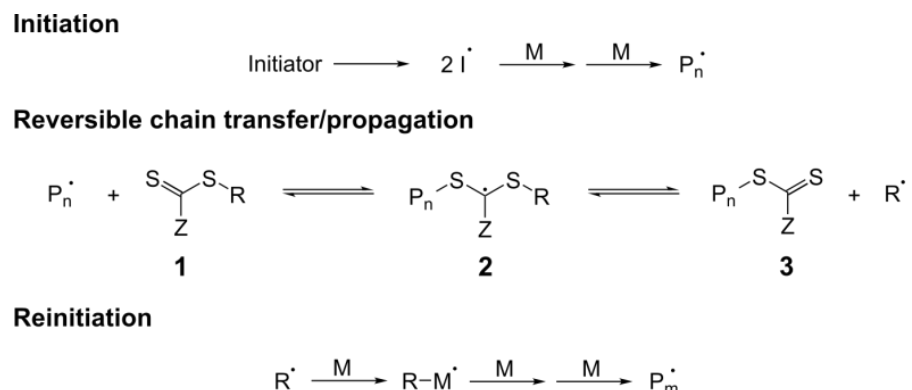

Chain equilibration/propagation

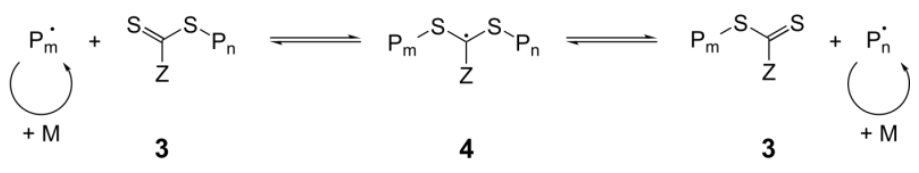

Termination

$$
\mathrm{P}_{\mathrm{m}}^{\cdot}+\mathrm{P}_{\mathrm{n}}^{\cdot} \longrightarrow \text { dead polymer }
$$

Scheme 2.1: Mechanism of RAFT polymerization. 
Linear multiblock copolymers are described as polymers containing more than three covalently bound polymer segments based on two or more different polymers or copolymer segments. In literature, the term "block" is often used ambivalent. For the sake of clarity, a block $b$ describes the polymeric chains between specific functional groups in this work. A segment $s$ denotes a part of a polymer chain with homogenous monomeric composition (Figure 2.2). According to this definition, the block number in the polymer is not altered by the second polymerization step. ${ }^{[15,38]}$

$$
b=3
$$

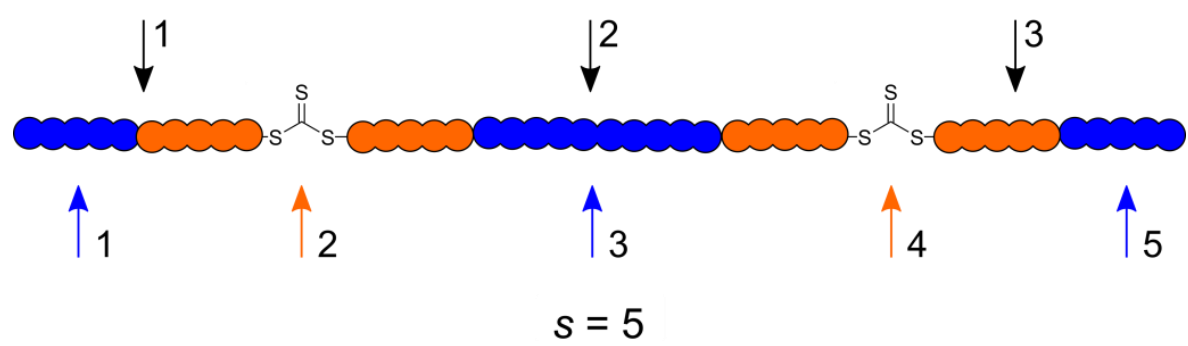

Figure 2.2: Illustration of the different meanings of the term blocks $b$ and segments $s$ by means of a polyfunctional RAFT agent.

The synthesis of those multiblock copolymers can be realized via three different strategies. The first and mostly used method is to interconnect preformed telechelic polymers. This strategy is versatile and can be applied easily to a wide range of different monomers. ${ }^{[46-54]}$ One disadvantage of this method is the great synthetic effort with at least three independent reactions. Furthermore, the low concentration of reactive end-groups does not allow to implement long segments $\left(<5000 \mathrm{~g} \cdot \mathrm{mol}^{-1}\right)$ or to synthesize high numbers of alternating segments. ${ }^{[55]}$ The second strategy is based on the sequential monomer addition. Commonly it is applied with anionic polymerization ${ }^{[56]}$ but also RAFT polymerization. ${ }^{[21,34]}$ There have been reports about synthesizing well-defined icosablock copolymers in a one-pot RAFT polymerization with the limitation to acrylamide.[32,33,57,58] But very recently, Zhang et al. developed a pathway for sequence controlled multiblock copolymers using acrylates.[21] The third approach relies on utilizing polyfunctional or cyclic RAFT agents. ${ }^{[15,59-66]}$ In this work, the focus will be on the use of polyfunctional RAFT agents with multiple RAFT groups along a main chain which will be discussed in detail in the following chapter. 


\subsubsection{Linear $(A B)_{n}$-Multiblock Copolymers via Polyfunctional RAFT Agents}

A polyfunctional RAFT agent exhibits multiple RAFT groups in one molecule. As a result, multiblock copolymers are easily accessible through two polymerization steps, as shown in Scheme 2.2. The great advantage is that this strategy can be applied to all monomer pairs that are amenable for radical polymerization. ${ }^{[38]}$ Motokucho et al. and You et al. were the first to prepare polyfunctional trithiocarbonates (polyTTC). ${ }^{[35-37]}$ Later, Brussels et al. used polydithiocarbamates to synthesize multiblock poly(n-butyl acrylate).[67-69] Ever since the publications increased on the topic of $(\mathrm{AB})_{\mathrm{n}}$-multiblock copolymers prepared via polyfunctional RAFT agents. ${ }^{[15,61,70]}$ There are also reports on amphiphilic multiblock copolymers, second-order nonlinear optical polymers, fluorescent polymers, photodegradable multiblock polystyrene and multiblock copolymers with enhanced mechanical properties.[60,71-76] Furthermore, gold-nanoparticle hybrid structures with multiblock polymers were published using the inherent benefit of polytrithiocarbonates groups with their high binding capacity to gold. $[20,59,77,78]$

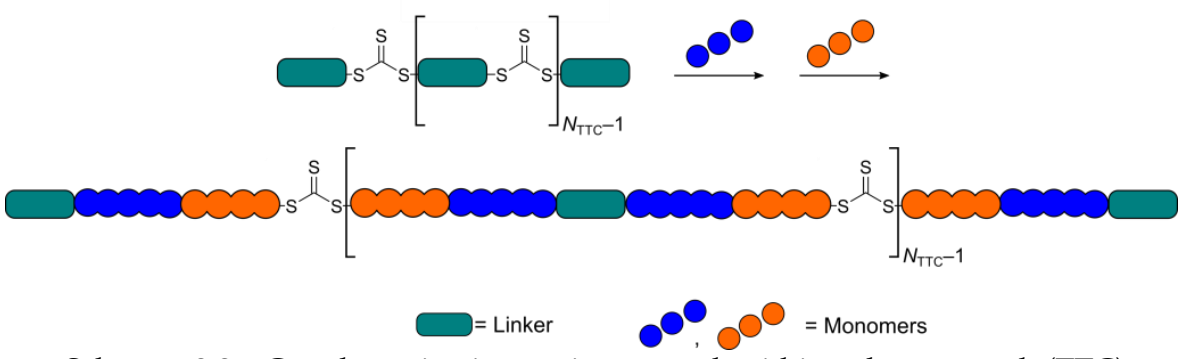

Scheme 2.2: Copolymerization using a polytrithiocarbonate poly(TTC) as functional RAFT agent for the synthesis of $(\mathrm{AB})_{n}$-multiblock copolymers. The variable $N_{\text {TTC }}$ denotes the number of TTC groups per molecule.

In general, the molar mass distribution of a multiblock copolymer containing multiple RAFT groups is rather broad in comparison to polymers with just one trithiocarbonate group. This observed broadening occurs due to the nature of the RAFT process. A constant reshuffling process appears until the system reaches its equilibrium state. Ebeling et al. defined a distribution function $N_{\mathrm{b}}$ for this equilibrium state, whereby $N_{\mathrm{b}}$ is the fraction of the molecules with $b$ blocks. They assumed an ideal model system with only one radical.[38] Termination reactions and other sidereactions are negligible, only reactions of the RAFT main equilibrium and addition of the monomer may occur. The radical species attacks a RAFT 
group of a multiblock polymer and generates a new radical species as shown in Scheme 2.3. Afterwards, a new growing radical is formed by splitting off another part of the molecule. In further reactions this radical can attack another molecule and so on. Refragmentation to the original species can be neglected due to no effect on the final block distribution. Moreover, two model mechanisms were proposed on how the radical will attack the RAFT groups. Model A assumes that every RAFT group in the system will be attacked with equal probability, independently of the macromolecules it belongs to. The second model B implies that every macromolecule is attacked with equal probability and then every RAFT group within this macromolecule has equal probability of being attacked. As a result of those presumptions two different distribution functions are determined:

$$
\begin{gathered}
N_{\mathrm{b}}^{\mathrm{A}}=\frac{1}{\bar{b}-1}\left(\frac{\bar{b}-2}{\bar{b}-1}\right)^{b-2} \\
N_{\mathrm{b}}^{\mathrm{B}}=\frac{4}{\bar{b}^{2}}\left(\frac{\bar{b}-2}{\bar{b}}\right)^{b-2}
\end{gathered}
$$

The average number of blocks $\bar{b}$ and segment $\bar{s}$ can be determined via the number of RAFT groups $N_{\text {TTC }}$ per molecule, which can be known from SEC characterization. ${ }^{[15]}$

$$
\begin{gathered}
\bar{b}=N_{\mathrm{TTC}}+1 \\
\bar{s}=2 \bar{b}-1
\end{gathered}
$$

Ebeling et al. also compared the ideal dispersities of the resulting distributions with the expected values for a coupling reaction of prepolymers. The distribution of those coupling reactions is based on the function of the Schulz-Flory-distribution. ${ }^{[79]}$ In Figure 2.3 the expected dispersities are displayed for the Schulz-Flory-distribution as well as for model A and B. The figure shows that the ideal dispersities reach a limit value of 2 for model A and 1.5 for model B, with an infinite number of blocks. According to this, expected dispersities of polymers prepared by polyfunctional RAFT agents will be greater than 1.5. Therefore, the experimental value of the dispersity should be between 1.5 and 2 .

Additionally, multiblock copolymers prepared by polyfunctional RAFT agents exhibit two distinct block species. These blocks cannot be 
interconverted during the redistribution process (see Figure 2.4). Blocks are defined as end blocks if they are connected with one end to a RAFT group. If blocks are connected to both ends to a RAFT group, they are called middle blocks. Therefore, middle blocks might display twice the chain length of end blocks depending on the polymerization system. Ebeling et al. showed in an experimental study the existence of distinct block species. They cleaved the RAFT groups of multiblock polystyrene sample which were prepared via polyfunctional RAFT agents. SEC measurements of the cleavage products revealed a bimodal distribution proving the presence of distinct block species.[15]

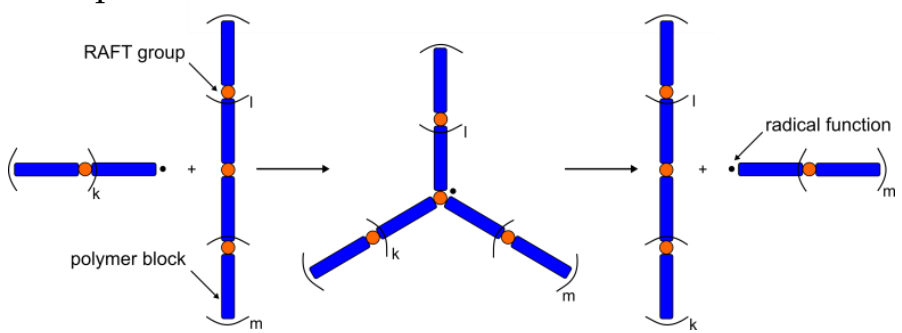

Scheme 2.3: Redistribution mechanism which takes place in presence of polyfunctional RAFT agents.[38]

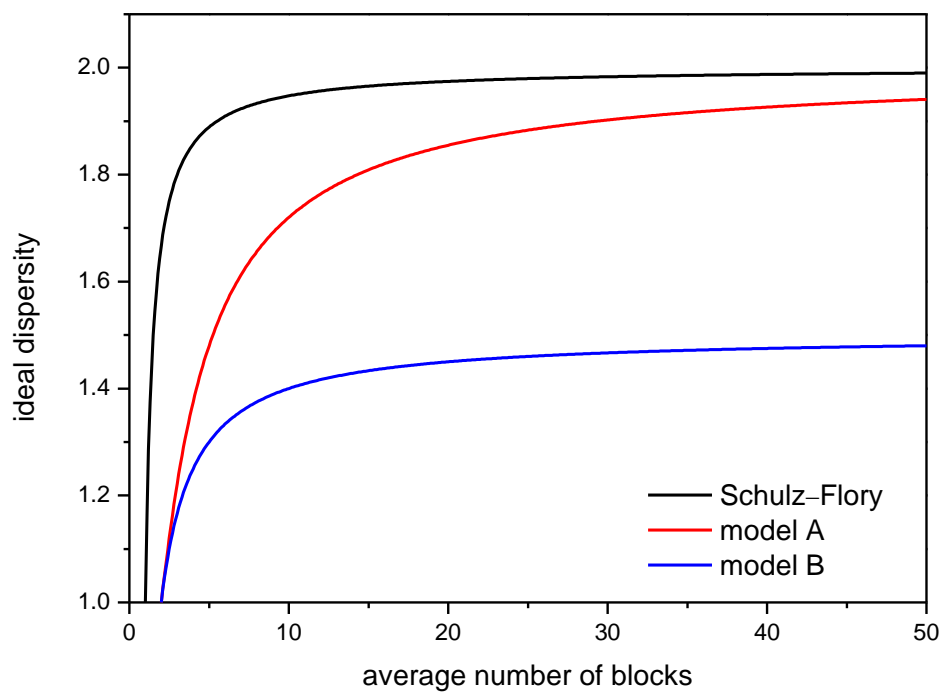

Figure 2.3: Comparison of ideal dispersities of multiblock copolymers synthesized via polyfunctional RAFT agent, which is located between the blue and red curve, with ideal dispersity of multiblock copolymers prepared by coupling of prepolymers. ${ }^{[38]}$ 


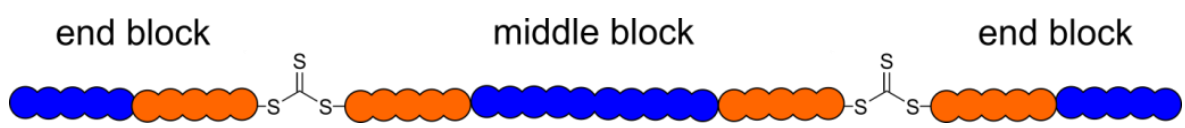

Figure 2.4. Illustration of the two block species which are obtained using a polyfunctional RAFT agent.

\subsection{Fundamentals of Microphase Separation of Block Copolymers}

Block copolymers containing two types of blocks, A and B, can undergo phase separation in the molecular scale of 5-100 $\mathrm{nm}$ into a variety of morphologies.[80] In contrast to polymer blends, the covalent bond of incompatible blocks prevents macroscopic phase separation. The microphase separation of block copolymers depends on three parameters: first, the volume fraction of $\mathrm{A}$ and $\mathrm{B}$ blocks $\left(f_{\mathrm{A}}+f_{\mathrm{B}}=1\right)$, secondly the total degree of polymerization $N$ and finally the Flory-Huggins interaction parameter $x$. This interaction parameter is inverse proportional to temperature (see equation (2.5) and describes the incompatibility between the blocks $\mathrm{A}$ and $\mathrm{B}$.

$$
\chi=\left(\frac{z}{k_{\mathrm{B}} T}\right)\left[\varepsilon_{\mathrm{AB}}-\frac{1}{2}\left(\varepsilon_{\mathrm{AA}}-\varepsilon_{\mathrm{BB}}\right)\right]
$$

Herein, $z$ is the number of the nearest neighbors per repeating unit in the polymer, $k_{\mathrm{B}}$ is the Boltzmann constant, $k_{\mathrm{B}} T$ the thermal energy and $\varepsilon_{\mathrm{AB}}$, $\varepsilon_{\mathrm{AA}}, \varepsilon_{\mathrm{BB}}$ are the interaction energies per repeating unit of $\mathrm{A}-\mathrm{B}, \mathrm{A}-\mathrm{A}$ and $\mathrm{B}-\mathrm{B}$.

Several studies have been developed dealing with the microphase separation of block copolymers exhibiting two segments.[81-87] Figure 2.5 shows the theoretical phase diagram predicted by self-consistent mean-field (SCMF) theory. ${ }^{[86,88]}$ It is based on the assumption that both monomers are equal in shape and size, as well as the fact that the polymer blocks exhibit the same Kuhn length $l^{\prime}$. ${ }^{[29,80]}$ The Kuhn length measures the stiffness of polymer $l^{\prime}=L N$. Herein, $L$ displays the contour length of a fully stretched polymer according to the freely-jointed-chain model and the number of monomers $N$.

The segregation product $\chi^{N}$ describes the degree of microphase separation. Increasing the temperature or decreasing $\chi N$ results in increasing the combinatorial entropy and leads to a disordered (homogenous) phase. This order-to-disorder transition (ODT) is marked with a dot in the diagram and the temperature at which this transition appears is referred to as $T_{\text {ODT }}$. Furthermore, a distinction is drawn between 
a weak segregation limit (WSL, $\chi N<10$ ) and a strong segregation limit (SSL $\chi N>>10)$. ${ }^{[89,90]}$ Figure 2.5 shows that, with increasing volume fraction $f_{\mathrm{A}}$ at a fixed $\chi N$ above the ODT $(\chi N \sim 10.5)$, order-to-order transition (OOT) appears from closely packed spheres (CPS) to body-centered cubic spheres $(S)$, hexagonally packed cylinders (C), bicontinuous gyroids $(G)$ and to lamellae (L) (see Figure 2.6). The morphologies are inverted when the composition is inverse.[91]

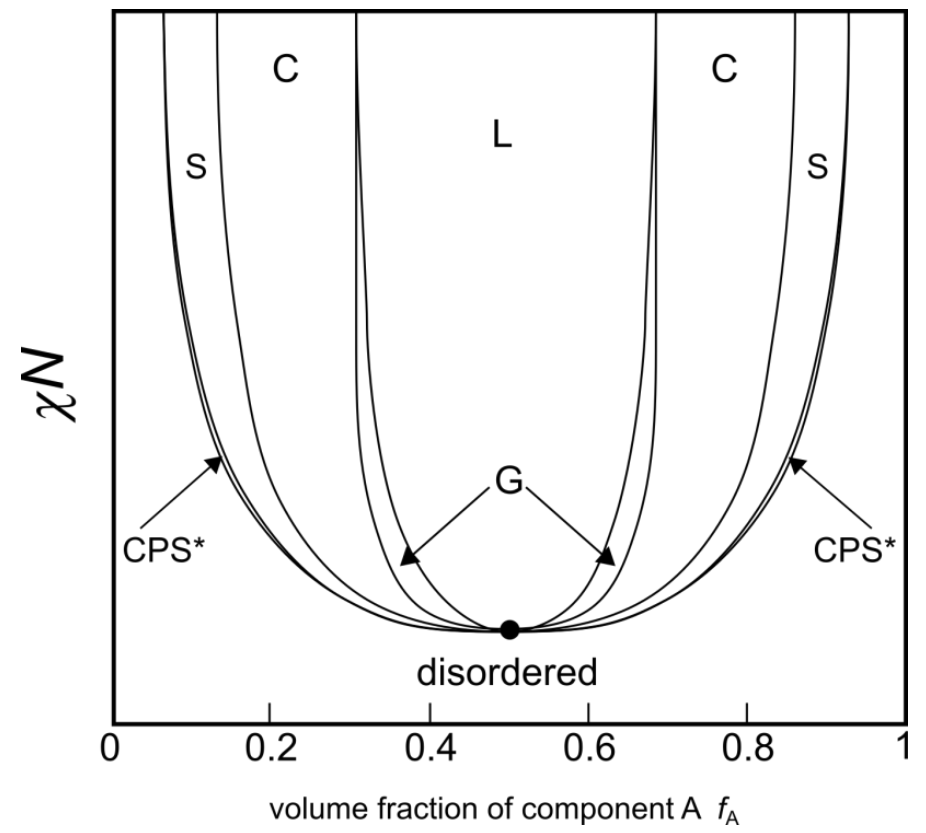

Figure 2.5. Theoretical phase diagram for linear AB block copolymers. Different equilibrium morphologies can be predicted depending on $\chi N$ and the volume fraction $f_{\mathrm{A}}$. Those morphologies are spherical $(\mathrm{S})$, close-packed spheres $\left(\mathrm{CPS}^{*}\right)$, hexagonally packed cylindrical (C), gyroid (G) and lamellae (L).
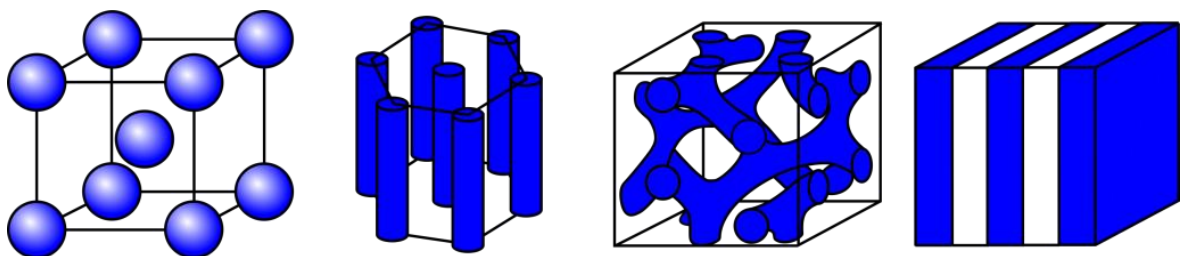

Figure 2.6. Equilibrium morphologies: spherical (S), hexagonally packed cylinders $(\mathrm{C})$, gyroid $(\mathrm{G})$ and lamellar $(\mathrm{L})$. 
Two factors are always competing in a microphase separation process. On the one hand, phase separation causes the chain stretching (entropic contribution) to move away from the preferred coiled polymer chain conformation. The degree of stretching depends on the volume fraction. On the other hand, the interfacial area of the two blocks is minimized in order to reduce the total interfacial energy (enthalpic contribution). In an asymmetric $\mathrm{AB}$ block copolymer $\left(f_{\mathrm{A}}<<f_{\mathrm{B}}\right)$, the interfacial area is curved (Figure 2.7a) and the configurational entropy is increased which leads to an energetically favorable sphere morphology. By increasing the volume fraction of $\mathrm{A}$ the curvature of the interfacial area decreases and the stretching is also reduced. The morphologies change from spheres to cylinders and to lamellae.[91]

(a)

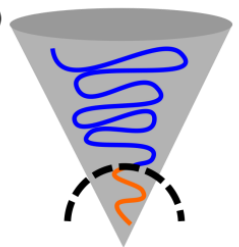

(b)

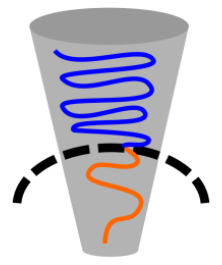

(c)

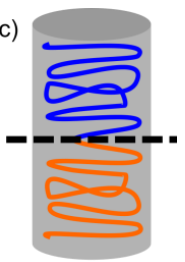

$f_{\mathrm{A}}$

Figure 2.7. Schematic illustration of the possible polymer chain arrangement in $\mathrm{AB}$ block copolymers for (a) sphere, (b) cylinder and (c) lamellae morphologies. The volume fraction $f_{\mathrm{A}}$ increases from left to right until $\sim 0.5$. The dash curve displays a part of the interface between the A and B domain. ${ }^{[91]}$

The introduction of more blocks into block copolymers results in an increased complexity for self-assembly in bulk. In theory, ABC linear block copolymers might possess 30 different morphologies.[80,92,93] Going further to higher block numbers in order to produce well-defined microphase separation is one of the great challenges of this decade. Experimental and theoretical studies have been published on this topic.[21-31,94] The next section highlights some key features of different theoretical studies, as microphase separation of $(\mathrm{AB})_{\mathrm{n}}$-multiblock copolymers is a central aspect within this work.

A theoretical phase diagram was predicated by Matsen and Schick using the SCMF theory. ${ }^{[29]}$ Earlier studies implied that with an AB repeating group greater than 10 the phase behavior will saturate to that of the infinite multiblock.[26,27] Their calculations were performed for a system consisting of an infinite, linear $(\mathrm{AB})_{\mathrm{n}}$-multiblock copolymer. Similar results to block copolymers exhibiting two segments have been found. Lamellar, hexagonal cylinder, spherical and gyroid microphases are stable (Figure 2.8). But the 
gyroid phase does not extend until the weak segregation limit. Furthermore, they investigated the influence of the Kuhn lengths of the monomers on the phase diagram. Unequal values lead to asymmetry of the phase diagram as shown in Figure $2.8 \mathrm{~b}$. The domain periodicity $D$ and the interfacial width $\lambda$ in the lamellar phase were studied as well (Figure 2.9). In the strong segregation limit, the same exponent dependency for $D$ and $\lambda$ as for $\mathrm{AB}$ block copolymers were found.[29]
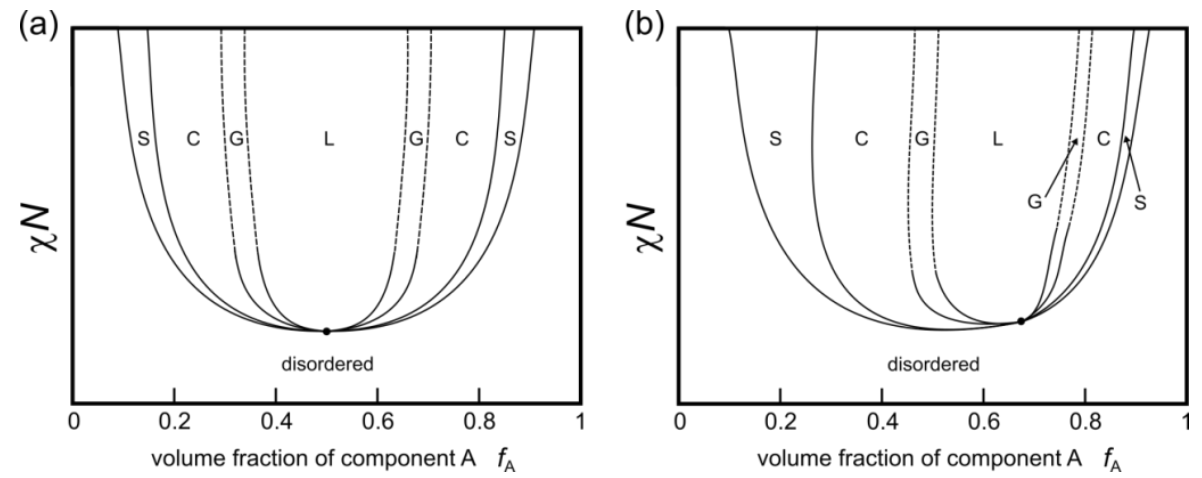

Figure 2.8. Phase diagram for a linear multiblock copolymer with (a) equal Kuhn length and (b) a ratio of Kuhn lengths of 1:2. The regions of stability for lamellae, gyroid, hexagonal cylinders and spherical phases are denoted by L, G, C and $S$ respectively. All transitions are first order except for the critical point which is marked by a dot. Dashed lines denote extrapolated phase boundaries.[29]

On the one hand, the order-disorder transition of symmetric multiblock for an infinite molar mass occurs at 7.55.[30,95,96] On the other hand, the microdomain formation is drastically slower compared to the corresponding $\mathrm{AB}$ block copolymers. Furthermore, the separated conformation of the multiblock copolymers can be described by loops and bridges (see Figure 2.9). The movement of a segment across an opposite domain exhibits a large free-energy barrier. Therefore, the establishment of the equilibrium of loops and bridges is a rather slow process.[96]

In addition, Zielinski and Spontak investigated the lamellar phase in the strong-segregation limit.[28] They consider only the molecular architecture within the strong segregation limit, as shown in Figure 2.9. The study revealed that microphase separation is favored by increasing the block number under constant block length. However, the tendency of microphase separation decreases with increasing block number but constant molecular weight and composition of the copolymer.[28] The weak-segregation limit of the lamellar phase was examined by Kavassails and Withmore.[27] 


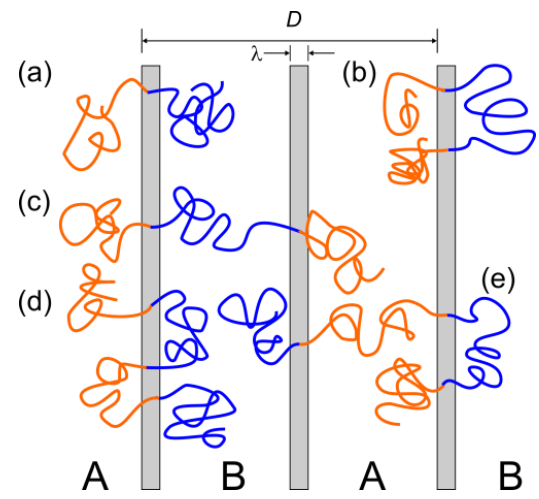

Figure 2.9. Schematic illustration of lamellar morphology of $(A B)_{n}$-block copolymers. (a) AB-block copolymer, symmetric ABA-block copolymer (b) looped and (c) bridged. $(\mathrm{AB})_{2}$-block copolymer can assume four conformations upon microphase separation (d) fully looped, (e) two in which one middle block is looped and the other block bridge and fully bridged (not displayed here). $D$ displays the domain periodicity and $\lambda$ the interfacial width. ${ }^{[28,94]}$

The most established techniques to gain microphase separation are thermal and solvent vapor annealing. ${ }^{\left[{ }^{[9-108]}\right.}$ In both methods chain mobility is increased by either raising the temperature above the glass transition temperature $T_{\mathrm{g}}$ or by solvent vapor which acts as a plasticizer on the polymer and reduces $T_{\mathrm{g}}$ of at least one of the blocks to go below room temperature. As a result, the equilibrium state of the ordered microdomains is promoted.[99]

The morphologies of thin films can be analyzed using a wide scope of techniques such as small-angle X-ray scattering (SAXS), transmission electron microscopy (TEM), scanning electron microscopy (SEM) and atomic force microscopy (AFM). In this work, the characterization of the microphase separation of multiblock copolymers is investigated via AFM. 


\subsection{Design of Multiblock Copolymers via Polyfunctional RAFT Agent}

In order to obtain microphase separation of multiblock copolymers, the individual segments need to be incompatible. Therefore, two monomersystems with different characteristics were selected. The first system exhibited different mechanical properties, e.g. glass transition temperature $T_{\mathrm{g}}$. This multiblock copolymer consisted of polystyrene (PS) and poly(n-butyl acrylate) (PBA) as individual segments. Polystyrene is known as a thermoplastic and a glassy polymer $\left(T_{\mathrm{g}}=100^{\circ} \mathrm{C}\right) \cdot{ }^{[82]} \mathrm{PBA}$ on the other hand is a rather soft, amorphous polymer with a glass transition temperature around $T_{\mathrm{g}}=-50^{\circ} \mathrm{C} \cdot{ }^{[109]} \mathrm{In}$ addition to that, this multiblock copolymer system has been thoroughly investigated by Ebeling et al. although the microphase separation was not explored.[15] The second system was an amphiphilic multiblock copolymer. For the first block of the multiblock copolymer, PS was chosen based on its hydrophobic nature. The second block exhibited the hydrophilic $N$-isopropylacrylamide (NIPAM) monomer. In literature, amphiphilic block copolymers are known to be a suitable structure directing agent in the preparation of mesoporous silica thin films. ${ }^{[110]}$ Styrene, BA and NIPAM as monomers have been extensively studied and demonstrated to be well-controlled via RAFT polymerization. $[15,20,42,111]$ Furthermore, microphase separation was already observed for $\mathrm{AB}$ block copolymers containing the selected monomers systems as building blocks.[18,99,112-114]<smiles>CCOCCOC(=O)C(C)SC(=S)SC(C)C(=O)CN(C)C</smiles>

polyRAFT

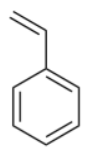

s<smiles>C=CC(=O)CCCC</smiles>

BA<smiles>C=CC(=O)NC(C)C</smiles>

NIPAM

Figure 2.10. Structures of the employed polyfunctional RAFT agent (polyRAFT) and monomers that were used for the design of microphase separated multiblock copolymers: Styrene (S), $n$-butyl acrylate (BA) and N-isopropylacrylamide (NIPAM).

The RAFT technique was chosen for the preparation of the multiblock copolymer. The usage of a polyfunctional RAFT, polyRAFT (see Figure 2.10), allows for the preparation of multiblock copolymers in a two-step polymerization (see section 2.2.2). The synthesis of this RAFT agent is based on the previous work of Ebeling et al. and Liu et al. Both showed that large

\footnotetext{
I The results which are reported in this section are partially of the bachelor thesis "Synthese und Charakterisierung von Multiblockpolymeren" by Dominik Ruhr.
} 
average block numbers (up to 17 and 20) are accessible by their syntheses.[15,37,61]

Scheme 2.4 shows the synthesis pathways. In both cases tetraethylene glycol 1 (TEG) was used as starting material. First (A) TEG was esterified with 2-bromopropionyl bromide 2 and triethylamine $\left(\mathrm{NEt}_{3}\right)$ in tetrahydrofuran (THF). Afterwards, the product 3 reacted with carbon disulfide $\left(\mathrm{CS}_{2}\right)$ and a hydroxyl-functionalized exchange resin, Ambersep ${ }^{\circledR} 900 \mathrm{OH}$, to the desired polyfunctional RAFT agent. The product can be fractionated due to the decreasing solubility of the produced trithiocarbonates with increasing chain length. In the work-up of the reaction the resin is first washed with carbon disulfide and then with THF, leading to two fractions with different numbers of trithiocarbonate groups (see Table 2.1). In reaction pathway (B) a polycondensation takes place. It is performed by reacting TEG with 2,2'-(thiocarbonylbis(sulfanediyl))dipropanoic acid 4 in cyclohexane.
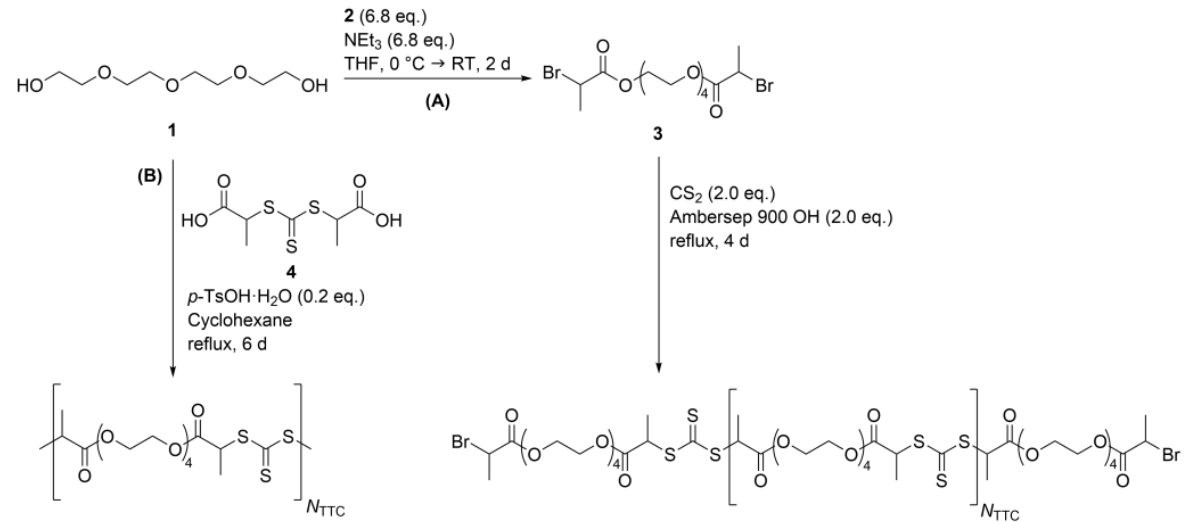

Scheme 2.4. Synthesis pathways derived from (A) Ebeling and (B) Liu et al.

The following table summarizes once more all the polyfunctional RAFT agents that are used within this work. In addition to the synthetic route, the mean number $N_{\text {TTC,SEC }}$ and $N_{\text {TTC,NMR }}$ of the trithiocarbonate groups, determined in each case by SEC and NMR, are given. The average number of trithiocarbonate groups was determined according to the methods used by Ebeling. ${ }^{[15]}$ Since the determination by these techniques is rather unprecise, the indicated values are only a rough approximation. The average number of trithiocarbonate groups of polyRAFT A was determined by SEC to $N_{\text {TTC,SEC }}=0.7$. This value is not physically possible because each molecule must contain at least one trithiocarbonate group, this value was set to 1.0 . 
Table 2.1. Overview of polyfunctional RAFT agents that were employed for further polymerizations.

\begin{tabular}{ccccc}
\hline polyRAFT & $\begin{array}{c}\text { Synthesis } \\
\text { pathway }\end{array}$ & Fraction & NTTC,SEC & $\boldsymbol{N}_{\mathrm{TTC}, \text { NMR }}$ \\
\hline A & $\mathrm{A}$ & $\mathrm{CS}_{2}$ & 1.0 & 2.5 \\
B & $\mathrm{A}$ & $\mathrm{THF}$ & 8.0 & 11.1 \\
$\mathrm{C}$ & $\mathrm{B}$ & - & 5.1 & - \\
\hline
\end{tabular}

In the following the control of the RAFT agent during radical polymerization will be assured by observing linear growth of the molar mass with monomer conversion. Furthermore, the control of the individual blocks will be investigated.

The provided polyfunctional RAFT agents A, B and C with a low, medium and high number of trithiocarbonate groups were employed in radical polymerization to form first multiblock homopolymers and afterwards multiblock copolymers. As mentioned above, styrene, BA and NIPAM were used as monomers. The synthesis strategy pursued in this work has already been explained in section 2.2.2. Accordingly, the polyfunctional RAFT agent (polyRAFT) was employed in two consecutive polymerization steps. In the first step, the multiblock homopolymers are synthesized and then used as macromolecular RAFT agents (macroRAFT) with the second monomer. The sequence of the used monomers is essential for a successful block copolymerization. The macroRAFT agent, synthesized in the first step, must have a high transfer constant compared to the second monomer. ${ }^{[115,116]}$ This means that the first blocks should be a better leaving group than the second one. Therefore, styrene was used in the first polymerization step and BA or NIPAM in the second step. All polymerizations were initiated thermally with azobis(isobutyronitrile) (AIBN) as initiator. The displayed concentrations of the polyRAFT were respectively calculated from the average molar mass $\bar{M}_{\mathrm{n}}$, which was determined via SEC. Since no appropriate calibration data for the polyfunctional RAFT agents was available, the SEC curves were measured using the calibration for linear polystyrene. This yielded rather low values of the molar mass. It can be assumed that the calculated concentrations were too high. However, all polyRAFT agents are based on the same method of calibration; it is one systematic error, which has the same effect for all carried out polymerizations. 


\subsubsection{Synthesis of Multiblock Polystyrene}

The prepared multiblock polystyrene samples were analyzed via SEC prior to use. Figure 2.11 shows the SEC curves of the bulk styrene polymerization with polyRAFT A. The SEC curves of the multiblock homopolymer show a good agreement of the RI and UV signal at a wavelength of $\lambda=310 \mathrm{~nm}$ at which trithiocarbonate groups absorb.[117] This indicates the presence of RAFT groups along the polymer chains and thus the desired multiblock structure is realized. For the purpose of simplification, the SEC curves will be only displayed by the RI signal in the following section. Figure 2.11 also shows that the maximum of the molar mass distribution increases with polymerization time and thus monomer conversion to higher molar masses occurs. In addition, a shoulder peak appears at higher reaction times. This can be explained by the nature of the polyfunctional RAFT. The RAFT itself has a molar mass distribution (see Figure 2.11, A) and within which a mixture of RAFT agents with different numbers of trithiocarbonate groups is present in the solution. Due to mechanism of RAFT, the blocks possess an equal chain length but the corresponding polymers differ in the molar mass. As mentioned above (see section 2.2.2) the RAFT polymers are reshuffled during polymerization but obviously there are polymers with different numbers of trithiocarbonate groups and thus also a different number of blocks. Furthermore, additional peaks $\left(M<2000 \mathrm{~g} \cdot \mathrm{mol}^{-1}\right)$ are visible in the low molecular weight range and they display impurities of the used RAFT agent which can be seen in a direct comparison of the signals. This argument is also confirmed by the fact that these additional peaks of each sample always appear in the same position. For the determination of the dispersity and the number average molar mass $\bar{M}_{\mathrm{n}}$ only signals above $2000 \mathrm{~g} \cdot \mathrm{mol}^{-1}$ are taken into account. The corresponding results are displayed in Figure 2.11. The dispersity tends to decrease with monomer conversion. However, these values could be confusing as the two peaks, which are initially still superimposed on one another, separate with progressive reaction time and this would automatically result in a higher degree of dispersity. In general, dispersity lies between 1.6 and 2 which is in good agreement with literature. ${ }^{38]}$ Furthermore, it must be kept in mind that uncertainties in the SEC characterization of the polyfunctional RAFT agent may be effective which could result in a lower true molar mass of the polyRAFT and therefore a lower $\bar{M}_{\mathrm{n}}$ of the synthesized polymers. 


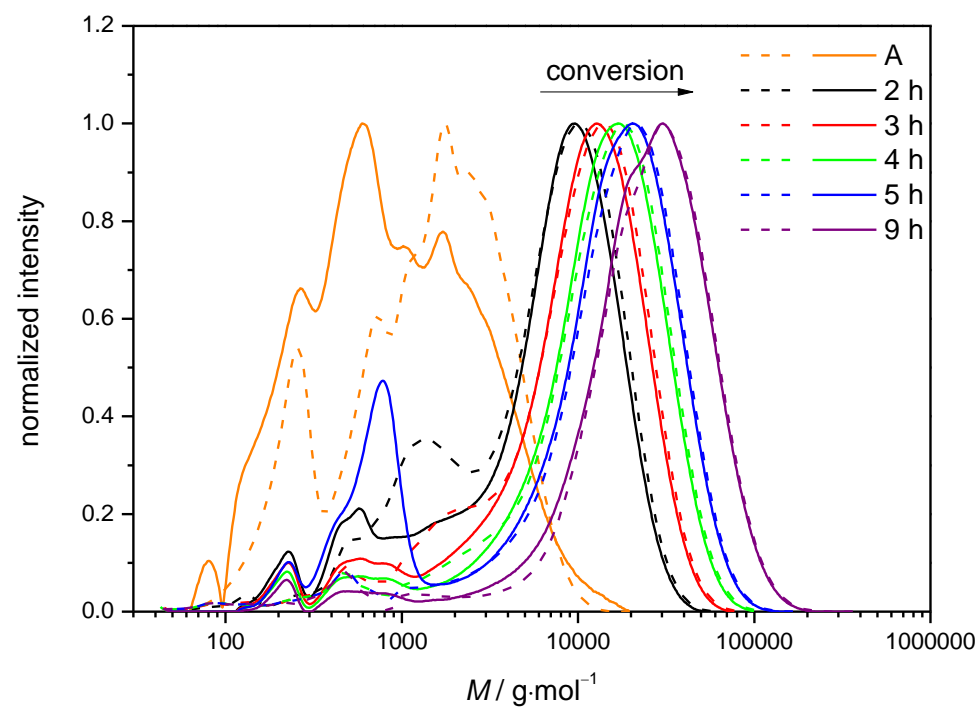

Figure 2.11. SE chromatograms (THF) of bulk polymerization of styrene and the polyRAFT $\mathrm{A}$ at $60^{\circ} \mathrm{C}, c_{\mathrm{RAFT}}=29.7 \mathrm{mmol} \cdot \mathrm{L}^{-1}, c_{\mathrm{AIBN}}=14.9 \mathrm{mmol} \cdot \mathrm{L}^{-1}$. UV (dashed line, wavelength of $\lambda=310 \mathrm{~nm}$ ) and RI (solid line) signal are displayed.

Furthermore, the influence of polymerization temperature was investigated. Polymerizations at $60^{\circ} \mathrm{C}, 90^{\circ} \mathrm{C}$ and $130{ }^{\circ} \mathrm{C}$ were performed with polyRAFT A. At a temperature of $130^{\circ} \mathrm{C}$, no thermal initiator was added to the polymerization solution due to the self-initiating character of styrene at elevated temperatures. It has to be mentioned that these experiments were also performed for the polyRAFT $\mathbf{B}$ and $\mathbf{C}$ which resulted in similar behavior and thus they are not displayed here. Figure 2.12 shows the direct comparison of the polymerization at different temperatures, for detailed polymerization conditions see Table 6.2. The number average molar mass $\bar{M}_{\mathrm{n}}$ and their dispersities $\oslash$ are plotted against the gravimetrically determined monomer conversion. As can be seen in the figure, the molar mass grows linearly with monomer conversion, which underlines the well-controlled behavior of the polyRAFT agent A. Furthermore, it shows that the increase of the molar mass with conversion for the polymerization at $60^{\circ} \mathrm{C}$, is more pronounced than the one for the polymerization at $90^{\circ} \mathrm{C}$. The polymerization without addition of AIBN (at $130^{\circ} \mathrm{C}$ ) is also well-controlled by the polyfunctional RAFT agent. The obtained molar masses are slightly higher than for the polymerization at $90^{\circ} \mathrm{C}$. Another important observation is the development of the dispersity. 
It is notable that the dispersity decreases during polymerization at $60^{\circ} \mathrm{C}$ while the dispersity increases during polymerization at $90^{\circ} \mathrm{C}$ and $130^{\circ} \mathrm{C}$. Their values are between 1.5 and 2 which is in a good agreement with the literature. Ebeling et al. already displayed the dependence of the dispersity with the average block number of multiblock copolymers. Therefore, the increase of the dispersity during polymerization with conversion is no evidence of a loss of control of the polyRAFT agent. The essential criterion of multiblock polymerization is the uniformity of the individual blocks. The cleavage of the multiblock (co)polymers and characterization of the cleaved products will be discussed below.

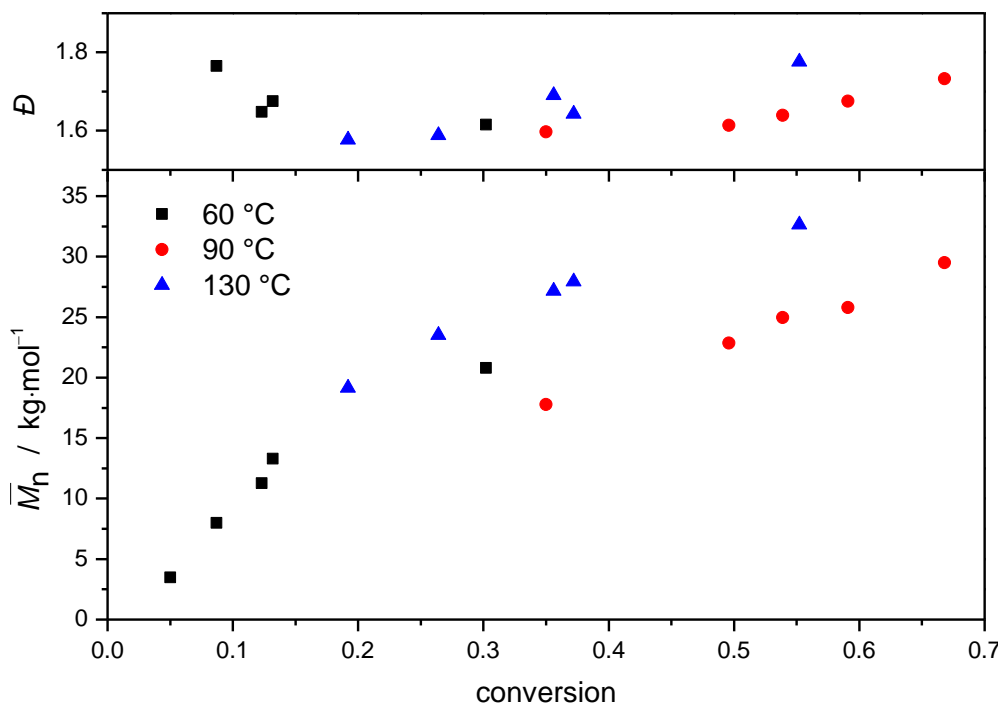

Figure 2.12. Comparison of bulk polymerization of styrene with polyRAFT A at $60{ }^{\circ} \mathrm{C}$ (black), $90^{\circ} \mathrm{C}$ (red) and ambient pressure with AIBN as initiator and $130{ }^{\circ} \mathrm{C}$ (blue) without any initiator, $c_{\mathrm{RAFT}}=29.7 \mathrm{mmol} \cdot \mathrm{L}^{-1}, c_{\mathrm{AIBN}}=14.9 \mathrm{mmol} \cdot \mathrm{L}^{-1}$.

Additionally, polyRAFT agent $\mathbf{B}$ and $\mathbf{C}$ were also polymerized at $130{ }^{\circ} \mathrm{C}$ in order to investigate the controlling behavior. Figure 2.13 shows the direct comparison of polymerization with the different RAFT agents. It reveals that all three polyRAFT are capable of controlling the radical polymerization of styrene. Polymerization with polyRAFT $\mathbf{C}$ exhibits a relative low increase in the molar mass. A possible explanation could be that the used polyRAFT was synthesized via polycondensation. The workup of the product was hardly possible. Thus, it can be assumed that the controlling agent was contaminated with starting materials which might 
influence the polymerization process. In general, no tendency of the dispersities with increasing trithiocarbonates groups inside the chain can be observed.

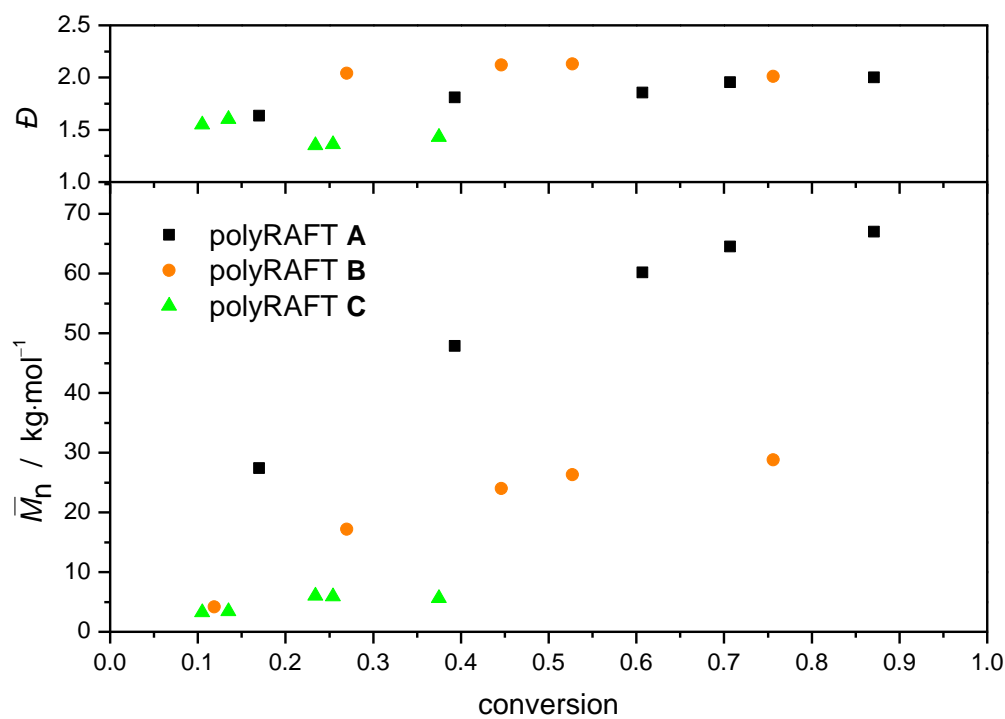

Figure 2.13. Comparison of the bulk polymerization of styrene using polyRAFT A (black), B (orange) and C (green) $\left(c_{\mathrm{RAFT}}=14.5 \mathrm{mmol} \cdot \mathrm{L}^{-1}\right)$ at $130^{\circ} \mathrm{C}$.

\subsubsection{Synthesis of Multiblock Copolymers}

For the synthesis of multiblock copolymers the previously prepared styrene polymer was employed as macromolecular RAFT agent in radical polymerization of either BA or NIPAM. The macromolecular RAFT agent was synthesized analogous to the polymerization with polyRAFT $\mathbf{B}$ at $130{ }^{\circ} \mathrm{C}$. This multiblock polystyrene exhibits a molar mass of $40000 \mathrm{~g} \cdot \mathrm{mol}^{-1}$ and a dispersity of 2.2. The experimental setup of the copolymerization was comparable to those of the styrene polymerization at $60^{\circ} \mathrm{C}$ (for detailed polymerization conditions see Table 6.4). Due to low solubility of the macromolecular styrene in BA, the polymerization was performed in solution (toluene). However, NIPAM is a solid monomer which needs to be polymerized in solution (DMF). It has to be noted that the displayed SEC 
curves and data gained from them might deviate from the true values due to the lack of appropriate SEC calibration data for the synthesized multiblock polystyrene. Therefore, the calibration for linear polystyrene was used. The monomer conversion was determined gravimetrically. In the following, the copolymerization of BA will be discussed and fully characterized. Figure 2.15 displays the SEC curves for the samples of copolymerization 1. Clearly, the molar mass grows with monomer conversion. Furthermore, no tailing can be observed in the SEC curves which indicate that there is no incomplete reinitiation present. Since most polymer chains contain several trithiocarbonate groups, at least one of them reinitiates the polymerization. ${ }^{[15]}$ Surprisingly, the first sample of the copolymerization shows a lower molar mass than the macromolecular RAFT agent, multiblock polystyrene ( $m$-PS) (see Figure 2.14). The molar mass with $23000 \mathrm{~g} \cdot \mathrm{mol}^{-1}$ is roughly half of the macromolecular RAFT agent $\left(40000 \mathrm{~g} \cdot \mathrm{mol}^{-1}\right)$. This phenomenon can be explained by the reshuffling mechanism of the RAFT process as described in section 2.2.2. Moreover, the dispersities of the multiblock copolymers are relatively high with values around 2.4. In the upcoming section the uniformity of the individual blocks of copolymers will be investigated to confirm the control of the RAFT agent.

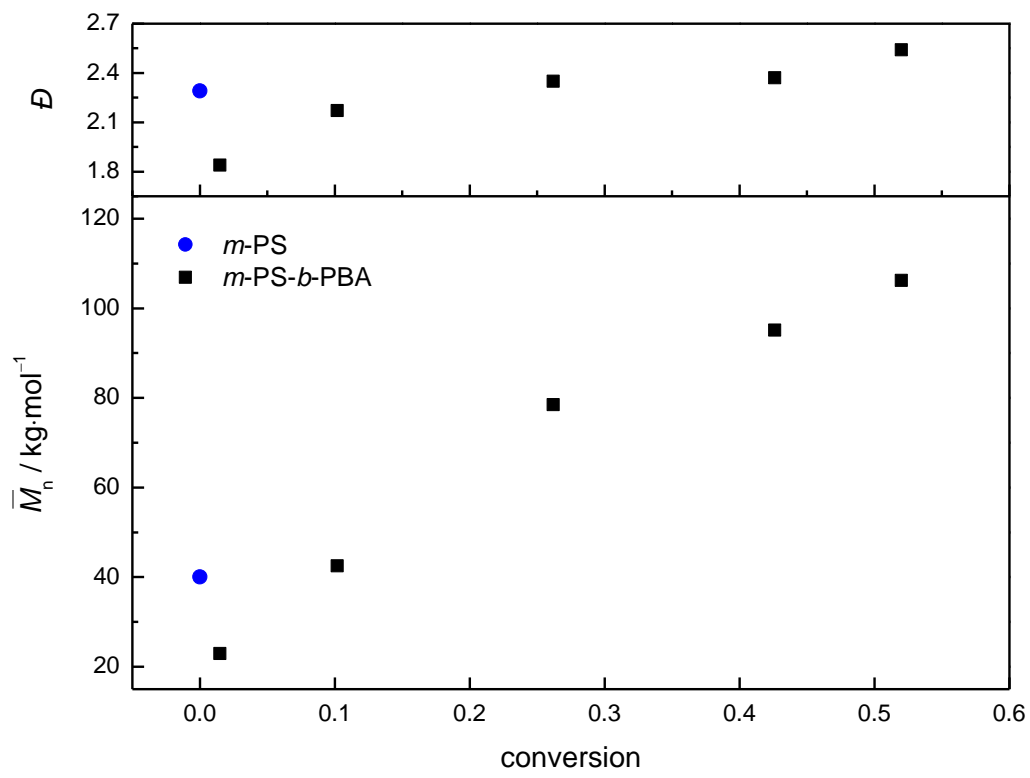

Figure 2.14. $\bar{M}_{\mathrm{n}}$ and dispersity as a function of monomer conversion for the copolymerization 1 of BA with styrene macroRAFT agent in toluene at $60^{\circ} \mathrm{C}$ and ambient pressure 


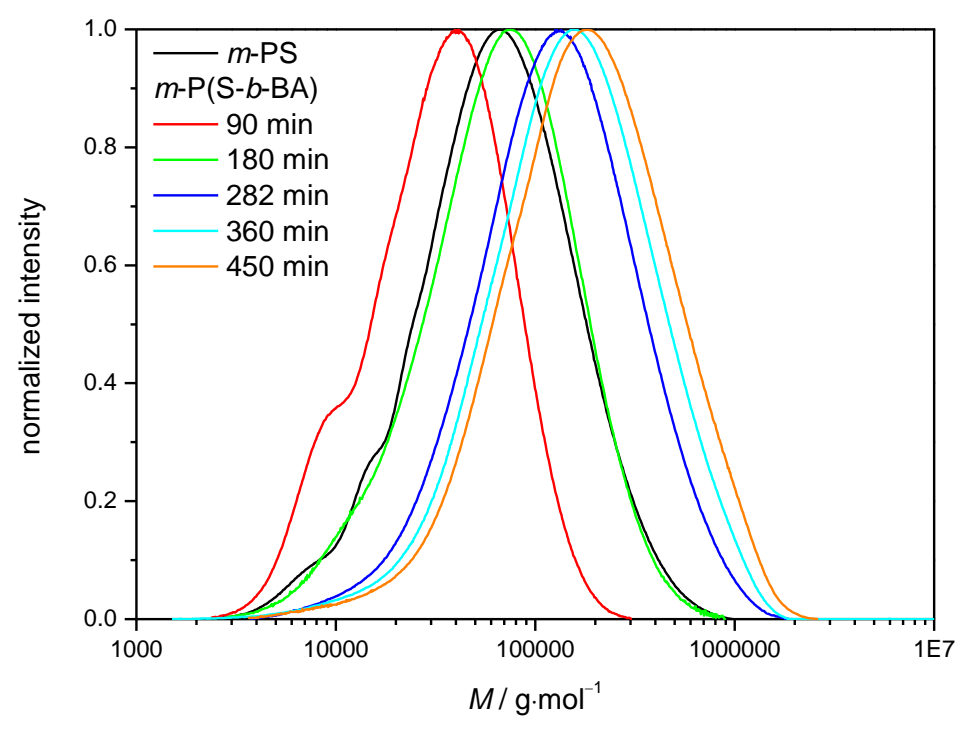

Figure 2.15. SEC curves of multiblock polystyrene (m-PS) and resulting multiblock copolymers with BA in the copolymerization $1 \mathrm{~m}$-(PS- $b$-PBA).

Additionally, the comonomer compositions of the multiblock copolymers were characterized via ${ }^{1} \mathrm{H}-\mathrm{NMR}$ spectroscopy. Figure 2.16 shows a representative spectrum of the multiblock copolymer. For calculation of the comonomer ratio $\phi_{\mathrm{PBA}}$ (ratio of BA to styrene), the integral of the signal of the aromatic protons of styrene (c, $I_{2}=6.2-7.5 \mathrm{ppm}$ ) was normalized to five protons. Afterwards, the following equation can be applied:

$$
\phi_{\mathrm{PBA}}=\frac{0.5 I_{1}}{1+0.5 I_{1}}
$$

The integral $I_{1}$ correlates with the two protons of the $n$-butyl alkyl chain neighboring to the ester group ( $f$, see Figure 2.16). The resulting compositions are summarized in Table 2.2. 


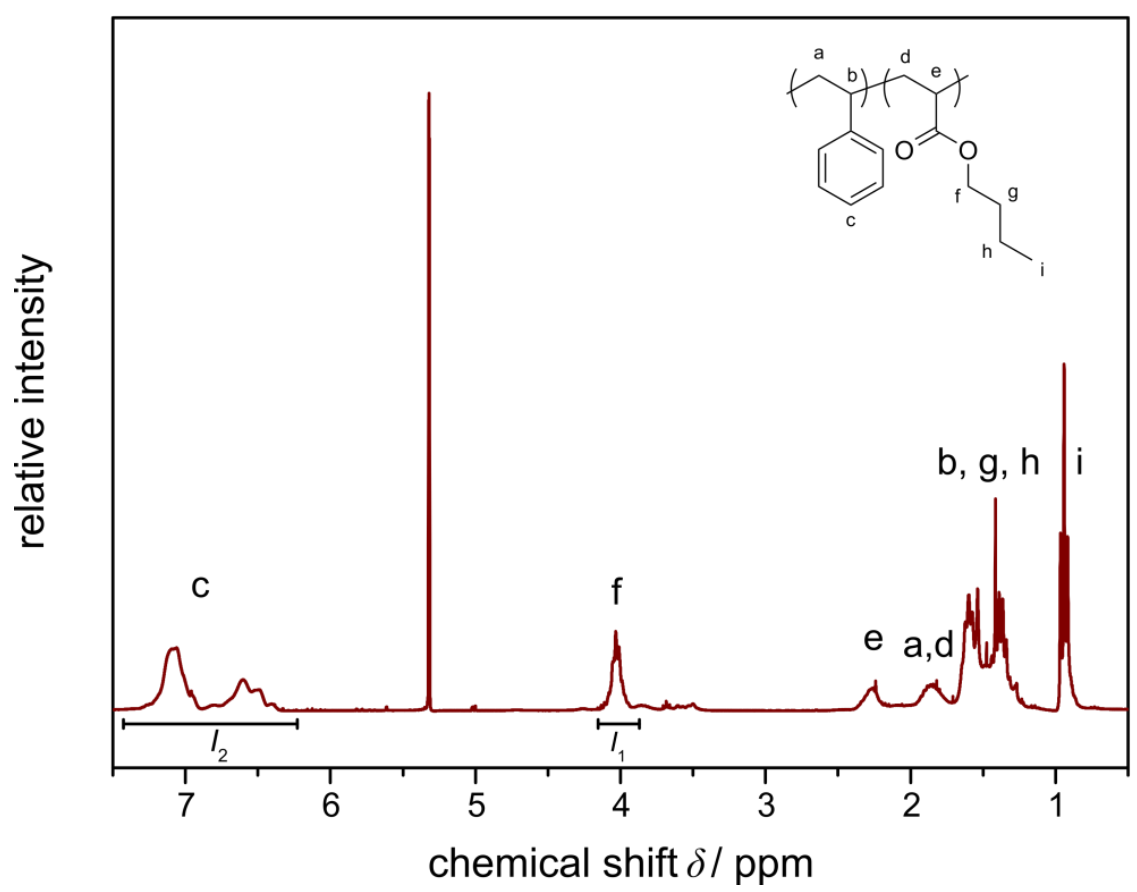

Figure 2.16. ${ }^{1} \mathrm{H}-\mathrm{NMR}$ spectrum of $m$-(PS-b-PBA) in $\mathrm{CD}_{2} \mathrm{Cl}_{2}$ with assignment of proton signals. The integrals used in equation (2.6) are shown with $I_{1}=3.7-4.2 \mathrm{ppm}$ and $I_{2}=6.2-7.5 \mathrm{ppm}$.

In order to establish a fully characterization of the multiblock copolymers the uniformity of the individual blocks was analyzed. The multiblock polymers and copolymers were cleaved at the trithiocarbonate groups. The resulting molar masses of the polymers prior to $\left(\bar{M}_{\mathrm{n}, 0}\right)$ and after $\left(\bar{M}_{\mathrm{n}, 1}\right)$ the cleavage can be determined by SEC. Ebeling et al. proposed the following equation to calculate the average number of trithiocarbonate groups:[15]

$$
N_{\mathrm{TTC}}=\frac{\bar{M}_{\mathrm{n}, 0}}{\bar{M}_{\mathrm{n}, 1}}-1
$$

Here, the cleavage of the trithiocarbonate groups was realized by an excess of radicals using AIBN (see Scheme 2.5). This procedure is known to avoid oxidative coupling of thiol groups during cleavage reaction. ${ }^{[118]}$ The cleaved individual blocks reveal the control of the RAFT process. SEC analyzation of the cleaved polymer implies an effective control, as the dispersities of the individual blocks are below 1.6 (see Table 2.2). 
Furthermore, the dispersities show a tendency to increase during copolymerization. Figure 2.17 illustrates selected SEC curves of the cleavage products and their corresponding multiblock copolymers.

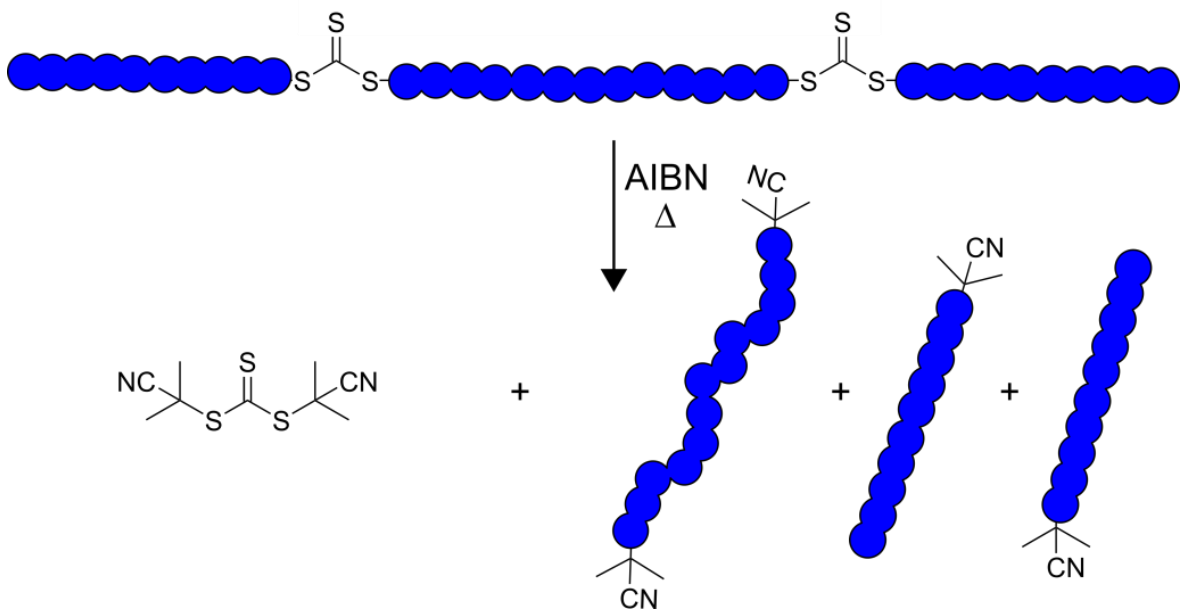

Scheme 2.5. Schematic illustration of the radical cleavage using an excess of AIBN.

Table 2.2. Overview of the $m$-(PS- $b$-PBA) copolymerization 1. SEC was measured versus linear polystyrene standards in THF. $\bar{M}_{\mathrm{n}, 0}$ denotes number average molar mass prior to cleavage of the multiblock copolymer and $\bar{M}_{\mathrm{n}, 1}$ after cleavage, $t$ reaction time and $U$ monomer conversion. The comonomer composition $\phi_{\mathrm{PBA}}$ (BA:Styrene) was determined by ${ }^{1} \mathrm{H}-\mathrm{NMR}$ and the average number of blocks $\bar{b}$ and segments $\bar{s}$ via equation (2.3) and (2.4), only integer numbers of the $N_{\text {TTC }}$ were used for calculation.

\begin{tabular}{cccccccccc}
\hline & $\boldsymbol{t} / \mathbf{m i n}$ & $\begin{array}{c}\overline{\boldsymbol{M}}_{\mathrm{n}, \mathbf{0}} / \mathbf{m} \\
\mathbf{g} \cdot \mathbf{m o l}^{-\mathbf{1}}\end{array}$ & $\boldsymbol{\Xi}$ & $\begin{array}{c}\overline{\boldsymbol{M}}_{\mathrm{n}, \mathbf{1}} / \\
\mathbf{g} \cdot \mathbf{m o l}^{-\mathbf{1}}\end{array}$ & $\boldsymbol{\Xi}$ & $\boldsymbol{\phi}_{\text {PBA }}$ & $\boldsymbol{N}_{\mathrm{TTC}}$ & $\overline{\boldsymbol{b}}$ & $\overline{\boldsymbol{s}}$ \\
\hline $\mathbf{1 a}$ & 180 & 42500 & 1.84 & 15000 & 1.35 & 0.44 & 1.8 & 3 & 5 \\
$\mathbf{1 b}$ & 282 & 78500 & 2.17 & 24300 & 1.42 & 0.68 & 2.2 & 3 & 5 \\
$\mathbf{1 c}$ & 360 & 95100 & 2.35 & 22200 & 1.55 & 0.66 & 3.5 & 5 & 9 \\
$\mathbf{1 d}$ & 450 & 106200 & 2.54 & 26400 & 1.60 & 0.75 & 3.0 & 4 & 7 \\
\hline
\end{tabular}




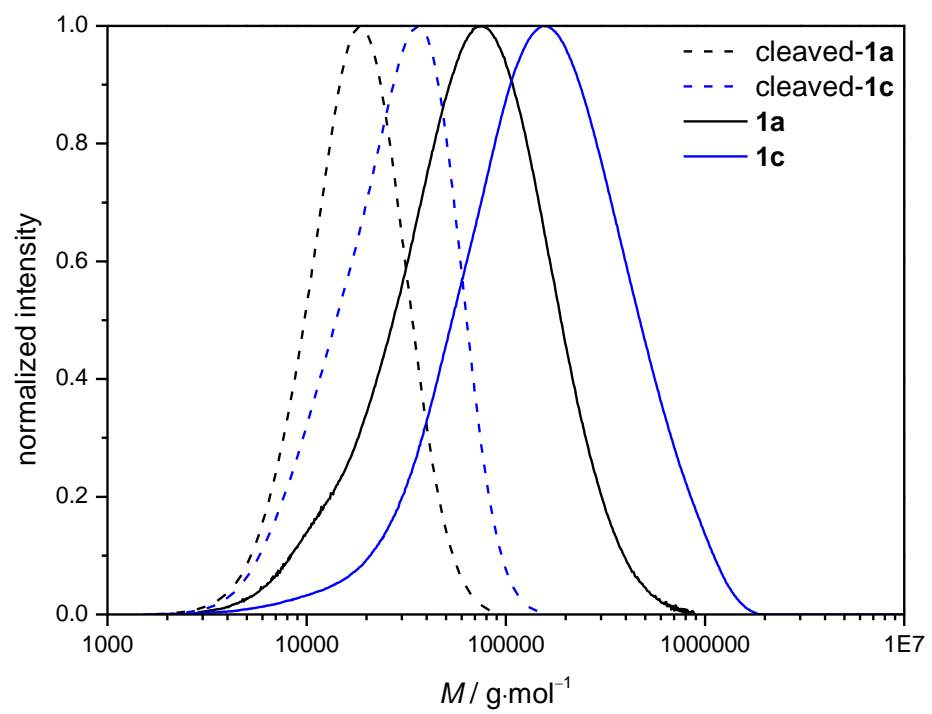

Figure 2.17. SE chromatograms (THF) of multiblock copolymers 1a and 1c (solid line) and their corresponding cleavage products (dashed line).

The copolymerization 2 of NIPAM was analyzed analogous to copolymerization $\mathbf{1}$ of BA (for detailed polymerization conditions see Table 6.5). The results will only briefly be discussed below. Due to solubility problems at higher molar masses the prepared copolymers were measured via SEC with $\mathrm{N}, \mathrm{N}$-dimethylacetamide (DMAc) as eluent. Table 2.3 shows that the molar mass grows with monomer conversion. But the dispersities are rather high with $Đ>2$. After cleavage of the multiblock copolymers the dispersities of the individual blocks are between 1.5 and 2.0. This means that the control of the RAFT agent decreases with increasing reaction time and monomer conversion respectively. As mentioned before, the comonomer composition was determined via ${ }^{1} \mathrm{H}-\mathrm{NMR}$ spectroscopy. A representative NMR spectrum is illustrated in Figure 2.18. Analogously to PBA the comonomer ratio $\phi_{\text {PNIPAM }}$ (ratio of NIPAM to styrene) was calculated. The integral of the signal of the aromatic protons of styrene (c, see Figure 2.18) and the proton of the amide group of NIPAM (f, $I_{2}=6.0-$ $7.5 \mathrm{ppm}$ ) was normalized to six protons. Afterwards, the following equation can be applied:

$$
\phi_{\mathrm{PNIPAM}}=\frac{I_{1}}{1+I_{1}}
$$


The integral $I_{1}$ correlates with the one proton of the isopropyl group ( $g$, see Figure 2.16). The resulting compositions are collected in Table 2.3.

Table 2.3. Overview of the $m$-poly(styrene- $b$ - $N$-isopropylacrylamide) copolymerization 2. SEC was measured versus linear polystyrene standards in DMAc. $\bar{M}_{\mathrm{n}, 0}$ denotes number average molar mass prior to cleavage of the multiblock copolymer and $\bar{M}_{\mathrm{n}, 1}$ after cleavage. The comonomer composition $\phi_{\text {PNIPAM }}$ (NIPAM:Styrene) was determined by ${ }^{1} \mathrm{H}-\mathrm{NMR}$ and the average number of blocks $\bar{b}$ and segments $\bar{s}$ via equation (2.3) and (2.4) only integer numbers of the $N_{\text {TTC }}$ were used for calculation.

\begin{tabular}{|c|c|c|c|c|c|c|c|c|c|c|}
\hline & $\begin{array}{c}t / \\
\min \end{array}$ & $\bar{U}$ & $\begin{array}{c}\bar{M}_{\mathrm{n}, 0} / \\
\mathrm{g} \cdot \mathrm{mol}^{-1}\end{array}$ & $\bar{\oplus}$ & $\begin{array}{c}\bar{M}_{\mathrm{n}, 1} / \\
\mathrm{g} \cdot \mathrm{mol}^{-1}\end{array}$ & 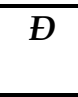 & $\phi_{\text {PNIPAM }}$ & $N_{\text {TTC }}$ & $\bar{b}$ & $\overline{\boldsymbol{S}}$ \\
\hline $2 a$ & 120 & 0.12 & 53060 & 2.28 & 15600 & 1.50 & 0. & 2.4 & 3 & \\
\hline $2 b$ & 240 & 0.44 & 61400 & 2.78 & 24200 & 1.63 & 0.7 & 1.5 & 3 & \\
\hline $2 c$ & 360 & 0.60 & 73500 & 2.50 & 26900 & 1.80 & 0.77 & 1.7 & 3 & 5 \\
\hline $2 d$ & 420 & 0.46 & 72360 & 2.37 & 25800 & 2.00 & 0.78 & 1.8 & 3 & $\pi$ \\
\hline
\end{tabular}

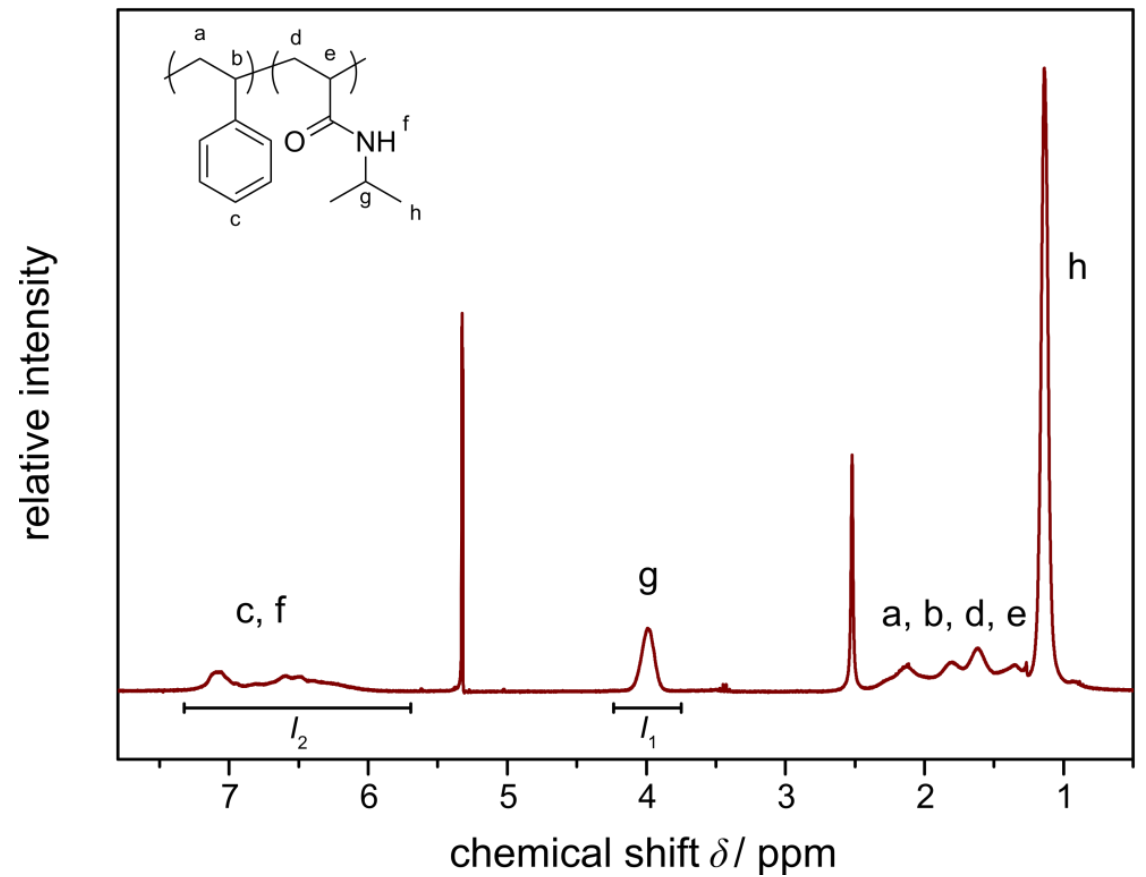

Figure 2.18. ${ }^{1} \mathrm{H}-\mathrm{NMR}$ spectrum of $m$-(PS- $b$-PIPAM) in $\mathrm{CD}_{2} \mathrm{Cl}_{2}$ with assignment of proton signals. The integrals used in equation (2.6) and (2.8) are shown with $I_{1}=3.7-4.2 \mathrm{ppm}$ and $I_{2}=6.0-7.5 \mathrm{ppm}$. 
Additionally, multiblock copolymers exhibiting a high number of blocks $(\bar{b}>5)$ were desired. For this purpose, polyfunctional RAFT agents introduced by Cavicchi et al. and Ebeling et al. were employed.[15,61] However, an unexpected result occurred. The number of polytrithiocarbonate groups decreased drastically after the first polymerization step with styrene. After a detailed literature review on the topic, a similar decrease in the number of polytrithiocarbonates within in the macromolecule is presented in the study of Li et al. ${ }^{[5]} \mathrm{A}$ higher number of blocks is excluded due to the rearrangement mechanism of the RAFT process.[38]

In summary, SEC revealed a good control over the polymerization of styrene and the copolymerization of BA. The control that could be achieved over the copolymerization of NIPAM, however, was only moderate. Furthermore, the multiblock character was verified with different techniques. In the following section will investigate microphase separation behavior of those multiblock copolymer systems.

\subsection{Investigation of the Microphase Separation of Multiblock Copolymer Systems via AFM}

Atomic force microscopy is an established technique for visualization of microphase separation. ${ }^{[99,119]}$ Not only can the height profile be measured but also the mechanical properties such as adhesion and Young's modulus. In the following sections the phase separation behavior of selected multiblock copolymers was investigated according to different parameter such as temperature, solvent and time.

\subsection{1 m-(PS-b-PBA) System}

From lamellar to hexagonally packed cylinders, different morphologies have been demonstrated for $\mathrm{AB}$ and $\mathrm{ABA}$ block copolymers containing PS and PBA as segments. ${ }^{[18,112,113]}$ In this section the multiblock copolymer system $m$-(PS- $b$-PBA) was investigated via differential scanning calorimetry (DSC) and AFM and their results described. First, a polystyrene multiblock polymer $m$-PS was synthesized using polyRAFT agent B. Subsequently, the polymer was chain-extended with BA monomer to form $m$-(PS- $b$-PBA) (for detailed polymerization conditions see section 6.7.1). This procedure gave multiblock copolymers with a constant PS segment length and increasing BA content. Detailed information on the polymers is collated in Table 2.4. The polymer composition was determined via ${ }^{1} \mathrm{H}-\mathrm{NMR}$ spectroscopy, as 
described in section 2.4. Using the densities $\rho_{\mathrm{PS}}=1.05 \mathrm{~g} \cdot \mathrm{cm}^{-3}$ and $\rho_{\text {PBA }}=1.08 \mathrm{~g} \cdot \mathrm{cm}^{-3}$ leads to the volume fraction $f_{\text {PBA }}$ of PBA. ${ }^{[99,120]}$ For the calculation of the average block number $b$ and segments $s$ only integer numbers of $N_{\text {TTC }}$ were used.

Table 2.4. Multiblock copolymer samples for microphase separation investigation with increasing PBA content $\phi_{\mathrm{PBA}}$ (determined via ${ }^{1} \mathrm{H}-\mathrm{NMR}$ spectroscopy) or volume fraction $f_{\mathrm{BA}} \cdot \bar{M}_{\mathrm{n}, 0}$ denotes number average molar mass prior to cleavage of the multiblock copolymer and $\bar{M}_{\mathrm{n}, 1}$ after radical cleavage. For determination of the average number of blocks $\bar{b}$ and segments $\bar{s}$ equations (2.3) and (2.4) were employed.

\begin{tabular}{cccccccccc}
\hline Sample & $\begin{array}{c}\overline{\boldsymbol{M}}_{\mathbf{n}, \mathbf{0}} / \\
\mathbf{g} \cdot \mathbf{m o l}^{-\mathbf{1}}\end{array}$ & $\boldsymbol{\Xi}$ & $\begin{array}{c}\overline{\boldsymbol{M}}_{\mathbf{n}, \mathbf{1}} / \mathbf{1} \\
\mathbf{g} \cdot \mathbf{m o l}^{-\mathbf{1}}\end{array}$ & $\boldsymbol{\oplus}$ & $\boldsymbol{\Phi}_{\text {PBA }}$ & $f_{\text {PBA }}$ & $\boldsymbol{N}_{\text {TTC }}$ & $\overline{\boldsymbol{b}}$ & $\overline{\boldsymbol{s}}$ \\
\hline I & 78500 & 2.17 & 24300 & 1.42 & 0.68 & 0.67 & 2.2 & 3 & 5 \\
II & 95100 & 2.35 & 21200 & 1.55 & 0.69 & 0.68 & 3.5 & 5 & 9 \\
III & 106000 & 2.54 & 26400 & 1.60 & 0.75 & 0.74 & 3.0 & 4 & 7 \\
IV & 181000 & 1.96 & 62000 & 1.32 & 0.88 & 0.88 & 2.0 & 5 & 3 \\
\hline
\end{tabular}

First, DSC measurements were performed in order to determine the glass transition temperatures $\left(T_{\mathrm{g}}\right)$ of the individual blocks. The existence of two $T_{\mathrm{gs}}$ is the initial evidence that the multiblock copolymer is capable of microphase separation. The DSC curves and corresponding $T_{\mathrm{g}}$ are summarized in Figure 2.19 and Table 2.5. All $m$-(PS- $b$-PBA) samples exhibit a glass transition temperature close to $0{ }^{\circ} \mathrm{C}$ which results from the PBA segments. A trend is apparent that the $T_{\mathrm{g}}$ decreases with increasing PBA segment which is in good agreement with the Fox-Flory-equation.[121,122] In general, $T_{\mathrm{g}}$ are higher compared to pure PBA with $T_{\mathrm{g}}=-54^{\circ} \mathrm{C} .{ }^{[123]}$ Mobility of the PBA chain segments is reduced by the PS segments. For sample I-III, a second $T_{\mathrm{g}}$ was observed and could be assigned to PS segments.[123] Surprisingly, sample II exhibited a drastically higher $T_{\mathrm{g}}$ for the PS segments compared to sample I and III. The $T_{\mathrm{g}}$ is increased by the lower concentration of chain ends in the macromolecule.[124] 


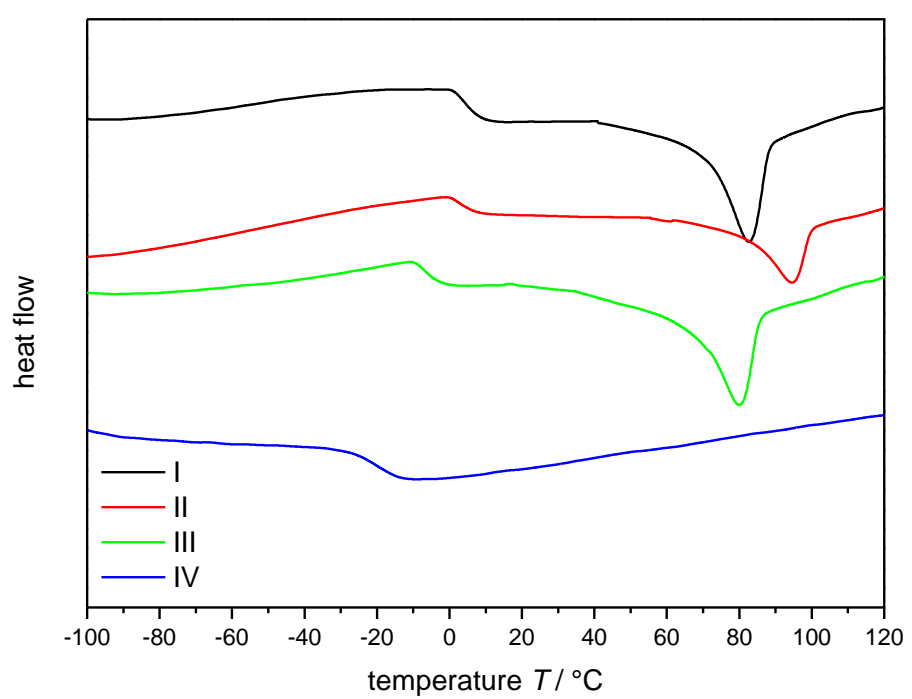

Figure 2.19. DSC curves of $m$-(PS- $b$-PBA) multiblock copolymers. Curves are shifted vertically for clarity. $T_{\mathrm{g}}$ that were determined are collated in Table 2.5.

Table 2.5. $T_{\mathrm{g}}$ of $m$-(PS- $b$-PBA) samples that were obtained from the respective DSC curves shown in Figure 2.19.

\begin{tabular}{ccc}
\hline sample & $T_{\mathrm{g}, 1} /{ }^{\circ} \mathrm{C}$ & $T_{\mathrm{g}, 2} /{ }^{\circ} \mathrm{C}$ \\
\hline I & 5 & 77 \\
II & 4 & 88 \\
III & -5 & 74 \\
IV & -19 & - \\
\hline
\end{tabular}

For the AFM investigations, thin polymer films were prepared via spin coating the multiblock copolymers $(2.5 \mathrm{wt} \%$ in propylene glycol methyl ether acetate, PGMEA) onto a glass substrate. Afterwards, the thin films were thermally annealed at $180^{\circ} \mathrm{C}$ for three days in a vacuum oven. The elevated temperature above the glass transition temperature of both blocks was necessary to obtain high chain mobility to form ordered microdomains. The AFM images were taken at different positions on the substrate and displayed homogenous orientation over the entire surface. Figure 2.20 shows the AFM topography image of the $m$-(PS- $b$-PBA) sample I. Dark areas (brown) correspond to lower regions and light areas (yellow) to higher regions in the AFM image. The image shows a smooth film surface. The composition of the multiblock copolymer and the almost equal Kuhn 
length of the polymer segments (PS $l^{\prime}=1.8 \mathrm{~nm}$ and PBA $l^{\prime}=1.7 \mathrm{~nm}$ ) indicate by the theoretical phase diagram (see section 2.3) lamellar or cylindrical as possible microstructures. ${ }^{[125]}$ Because of the lower surface energy of PBA, $\gamma_{\mathrm{PBA}}=31 \mathrm{mN} \cdot \mathrm{m}^{-1}$, than PS, $\gamma_{\mathrm{PS}}=41 \mathrm{mN} \cdot \mathrm{m}^{-1}$, the PBA block tends to segregate to the air interface and the PS block preferably to the substrate. ${ }^{[119,126]}$ As a result, no phase separation was observed for a lamellar structure due to the asymmetric wetting (one block prefers the substrate and the other the air interface). In theory, a smooth film will be obtained if the film thickness is $d=(n+0.5) L_{0}$. Herein, $L_{0}$ represents the lamellar period and $n$ a natural number. Holes or islands with a height of $L_{0}$ will occur if the film thickness is not compatible with the lamellar. ${ }^{[119]}$ However, no holeisland structure was observed which indicates a compatible film thickness. Another explanation for the smooth surface could be that the segregation product $\chi N$ is too low and results in a disordered phase. To exclude a disordered state and prove a lamellar morphology complementary studies via SAXS are necessary which were not performed within this work.

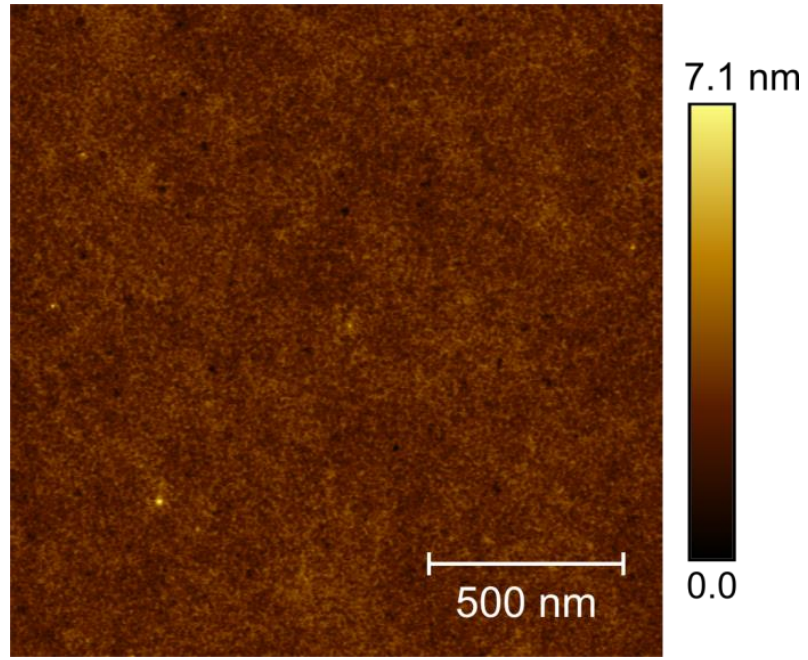

Figure 2.20. AFM topography of $m$-(PS- $b$-PBA), sample I, with $M_{\mathrm{n}}=78500 \mathrm{~g} \cdot \mathrm{mol}^{-1}$ and $f_{\mathrm{PBA}}=0.67$ after thermal annealing at $180^{\circ} \mathrm{C}$ for 3 days under vacuum.

The AFM image of sample II (see Figure 2.21), however, shows disordered micellar morphology as light dots appear within a dark matrix. This morphology can indicate either spheres or perpendicular cylinders. ${ }^{[99]}$ The inhomogeneity of the thin film leads to variation of the color scale along the AFM image and the microstructure is barely visible. Considering the volume fraction of PS $\left(f_{\mathrm{PS}}=0.32\right)$ the light dots are attributed to 
unordered cylinders of PS within a matrix of PBA. To determine the domain size of the cylinders, the power spectral density (PSD) function was used. The PSD function is the magnitude square of the Fourier spectrum of surface topography and contains information about the vertical and lateral distribution of the individual domain parts. The PSD can be calculated either from one-dimensionally recorded profiles (1D-PSD) or twodimensionally from surface data (2D-PSD). The surface topography of a sample can be interpreted as a two-dimensional function (horizontal and vertical), which can be decomposed into their spatial waves or spatial frequencies by means of the fast Fourier transformation (FFT). The AFM height profiles are evaluated using the 2D-PSD function of the NanoScope Analysis software and Figure $2.22 \mathrm{~b}$ shows a representative curve. To determine the domain size, the PSD was baseline corrected and the maximum of the function was fitted with a Lorentzian fit. The value of the domain size corresponds to the reciprocal value of the maximum. Sample II exhibited a domain size of $(26 \pm 19) \mathrm{nm}$.

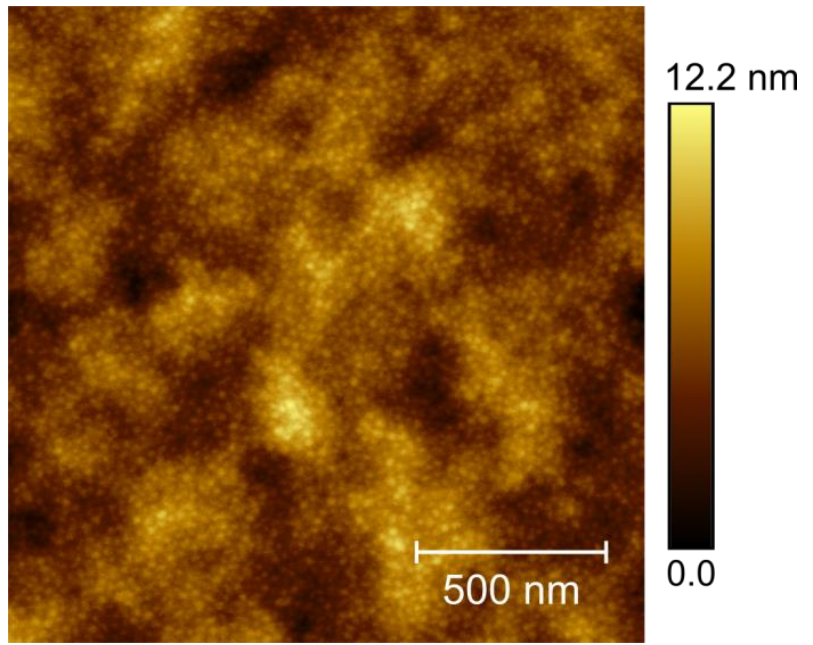

Figure 2.21. AFM topography of sample II with $M_{n}=95100 \mathrm{~g} \cdot \mathrm{mol}^{-1}$ and $f_{\mathrm{PBA}}=0.68$ after thermal annealing at $180^{\circ} \mathrm{C}$ for 3 days under vacuum.

After increasing the volume fraction of PBA, microphase separation can be observed within sample III. The AFM image of sample III shows wormlike structures. The light worm-shaped objects are incorporated into a dark matrix. The AFM image can be characterized as lying cylinders or as perpendicular lamella. The allocation of the different areas will be discussed below. Considering the volume fraction of $f_{\mathrm{PBA}}=0.74$ a cylindrical structure is more reasonable. The cylinders are packed with no preferential 
alignment. The domain spacing of those can be obtained from the positon of the peak in the PSD of the image (see Figure 2.22b). Due to roughness of the surface area the PSD distribution shows elevated values at frequencies around zero. The average domain spacing is $(20 \pm 10) \mathrm{nm}$. The structure shows irregularities. This grain boundary structure can be formed due to different reasons. The ordered structure nucleates at many different points and ordered regions will grow until a grain boundary wall is formed. On the other, substrate defects or impurities can interrupt the domain structure formation. ${ }^{[98]}$ The observed structure might not be in an equilibrium state. And final and most reasonable point, the system is in the weak segregation regime. ${ }^{[127]}$ In literature, it was shown that the number of those defects can be decreased by longer annealing times and increasing annealing temperature.[128] However, this effect could not be observed within this work (see Appendix A).
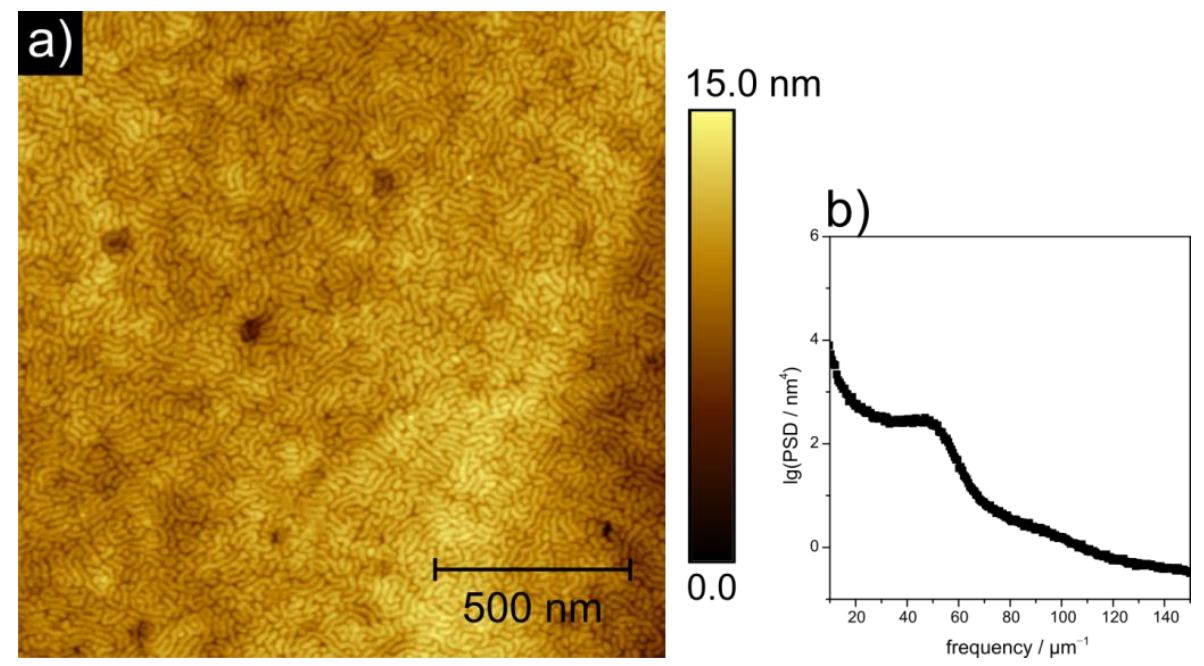

Figure 2.22. a) AFM topography of sample III with $M_{\mathrm{n}}=106200 \mathrm{~g} \cdot \mathrm{mol}^{-1}$ and $f_{\mathrm{PBA}}=0.74$ after thermal annealing at $180^{\circ} \mathrm{C}$ for 3 days under vacuum and b) twodimensional, isotropic PSD distribution of AFM image.

By increasing the volume fraction up to $f_{\mathrm{PBA}}=0.88$ (sample IV) the phase separation disappears. The AFM topography image shows (see Figure 2.23) a flat surface with unorganized light dots, no ordered structure is observed. The lack of a microstructure along with the finding of only one glass transition temperature indicates that the sample IV is not capable of undergoing phase separation. Furthermore, it can be assumed that the sample IV is in the region of the disordered phase based on the theoretical phase diagram (see Figure 2.8). In summary, phase separation was observed 
for a volume fraction $0.68 \geq f_{\mathrm{PBA}} \leq 0.74$ (see Table 2.6).

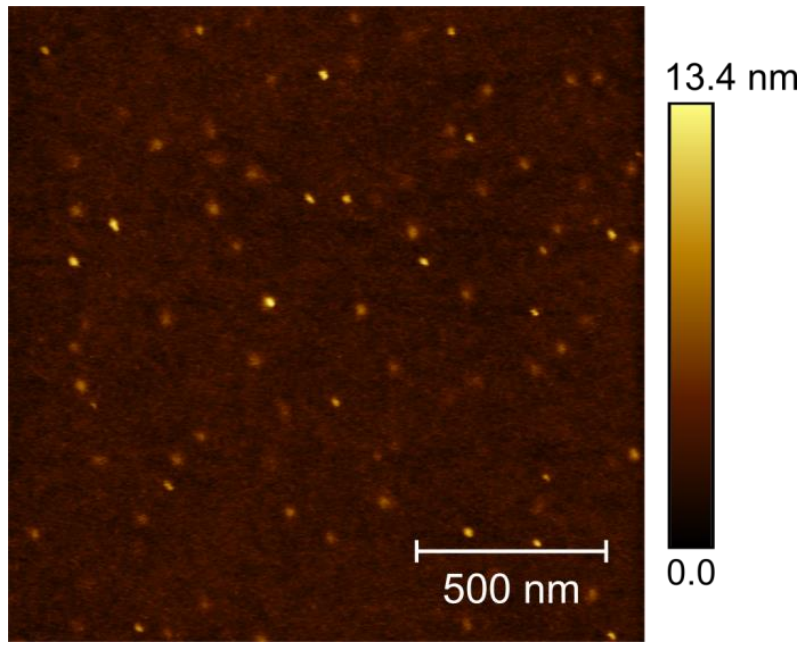

Figure 2.23. AFM topography of sample IV with $M_{\mathrm{n}}=181000 \mathrm{~g} \cdot \mathrm{mol}^{-1}$ and $f_{\mathrm{PBA}}=0.88$ after thermal annealing at $180^{\circ} \mathrm{C}$ for 3 days under vacuum

Table 2.6. Morphologies with respect to the sample surface of the synthesized $m$-(PS- $b$-PBA) as a function of the PBA volume fraction $f_{\mathrm{PBA}}$.

\begin{tabular}{ccc}
\hline sample & $f_{\text {PBA }}$ & morphology \\
\hline I & 0.67 & disordered \\
II & 0.68 & perpendicular cylinder \\
III & 0.74 & parallel cylinder \\
IV & 0.88 & disordered \\
\hline
\end{tabular}

Figure 2.24 shows a set of AFM images of sample III. The images were obtained using the PeakForce Quantitative Nano-Mechanics (PFQNM) imaging mode. Besides topography images, stiffness, Young's modulus, adhesion and energy dissipation of a sample surface can be provided. PFQNM is based on the peak force tapping mode which performs a fast force curve at every pixel in the image. The maximum force (peak force) on the tip is controlled in order to protect the tip and sample from damage. To obtain the properties of the sample the measured force-distance curves are used as feedback signals. $\left[{ }^{97,129,130]}\right.$ The local characterization of the Young's modulus and adhesion profiles allows to assign the domains to the corresponding segments. It has to be mentioned that the scale bars for those profile images are just a relative scale since no reasonable calibration is available with the used AFM. In the adhesion image (Figure 2.24a), light areas display a high adhesiveness and dark areas lower adhesiveness. Since 
the AFM measurements were performed at room temperature the PBA segments exhibit a higher adhesiveness than PS due to their lower glass transition temperature. This means that the worm-like objects can be assigned to PS segments and the matrix to PBA. In addition, the Young's modulus profile (see Figure 2.24b) shows areas of high and low Young's modulus. Here, the worm-like objects are lighter corresponding to a stiffer material than the surrounding matrix. Again the worm-like objects belong to PS which has a higher Young's modulus $(2.5 \mathrm{GPa})$ than PBA (6.4 MPa). ${ }^{[97,131] . ~}$
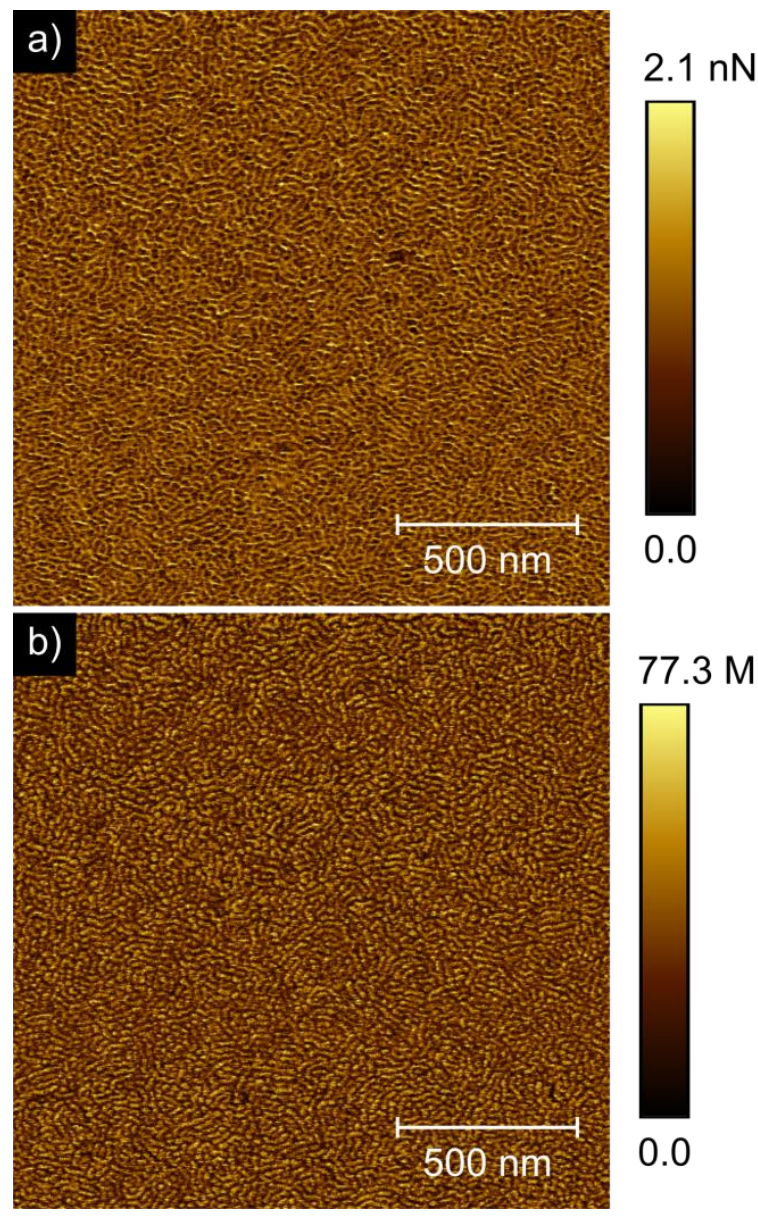

$77.3 \mathrm{MPa}$

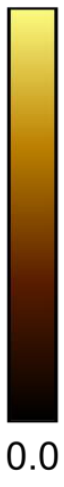

Figure 2.24. AFM profiles of sample III a) adhesion profile and b) Young's modulus profile measured via PFQNM. 
Further investigations of sample III were performed. The annealing temperature was varied successively from room temperature to $180{ }^{\circ} \mathrm{C}$, the respective AFM images can be found in the Appendix A. No microphase separation was observed below $180^{\circ} \mathrm{C}$ as annealing temperature. It can be reasoned that high temperature is necessary to reach the suitable chain mobility for microphase separation.

Moreover, solvent annealing was performed using chloroform and tetrahydrofuran (THF) as solvent. It was expected that a different morphologies would be accessible. Solvent annealing increases the chain mobility by reducing the $T_{\mathrm{g}}$ of at least one of the blocks below room temperature. The chosen solvents can be considered as good solvent for both blocks.[132] In literature, it has been shown that the choice of solvent for annealing allows to control the self-assembled morphology.[108] The AFM topography images (see Figure 2.25) show a smooth surface with some random organized lighter areas. No long-range order could be observed. The lack of self-assembly can be explained by lower $\chi N$ resulting from the solvent annealing. Another explanation is that the selective swelling of one block occurs leading in a different volume fraction and thus a different area in the phase diagram.
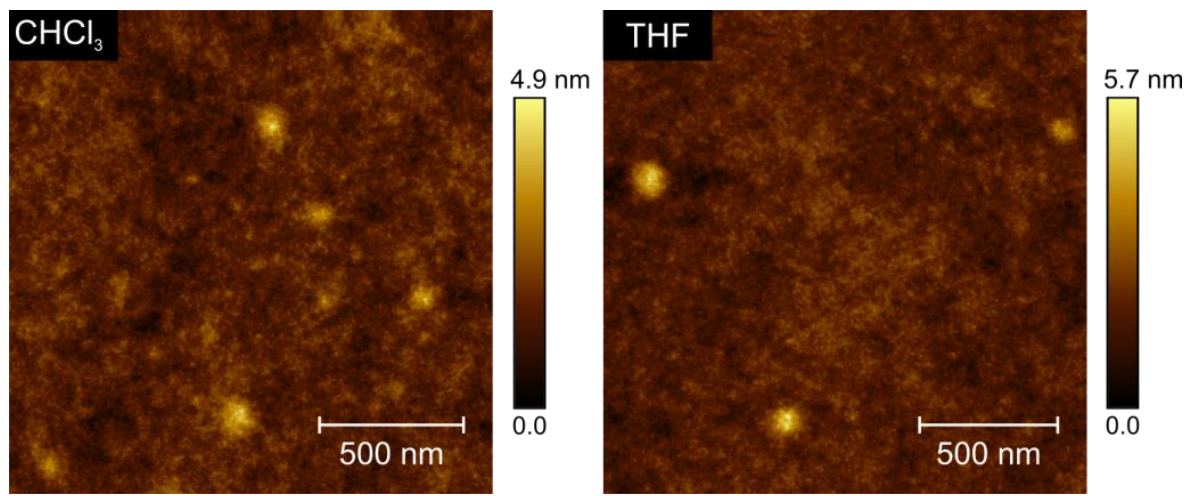

Figure 2.25. AFM topography images of sample III after solvent annealing using chloroform (left) and THF (right) for $24 \mathrm{~h}$.

Of further interest was the comparison of the multiblock polymer with their corresponding cleaved block copolymers. The multiblock copolymer III was cleaved by an excess of radicals (see Scheme 2.5). The cleavage product has a molar mass of $26400 \mathrm{~g} \cdot \mathrm{mol}^{-1}$ and a rather high dispersity of 1.6. A thin film of this block copolymer was prepared, thermally annealed in the same manner as sample III and then analyzed via AFM. The resulting AFM topography image is displayed in Figure 2.26a. It has to be mentioned 
that height scale of the images was not aligned to zero in order to keep a high color contrast of the structure. Short worm-like objects embedded in a darker matrix can be observed. PSD revealed a domain size with a value of $(22 \pm 18) \mathrm{nm}$ (see Figure 2.26b). Consequently, phase separation is possible for multiblock copolymers with a block and segment number of 4 and 7 , respectively.
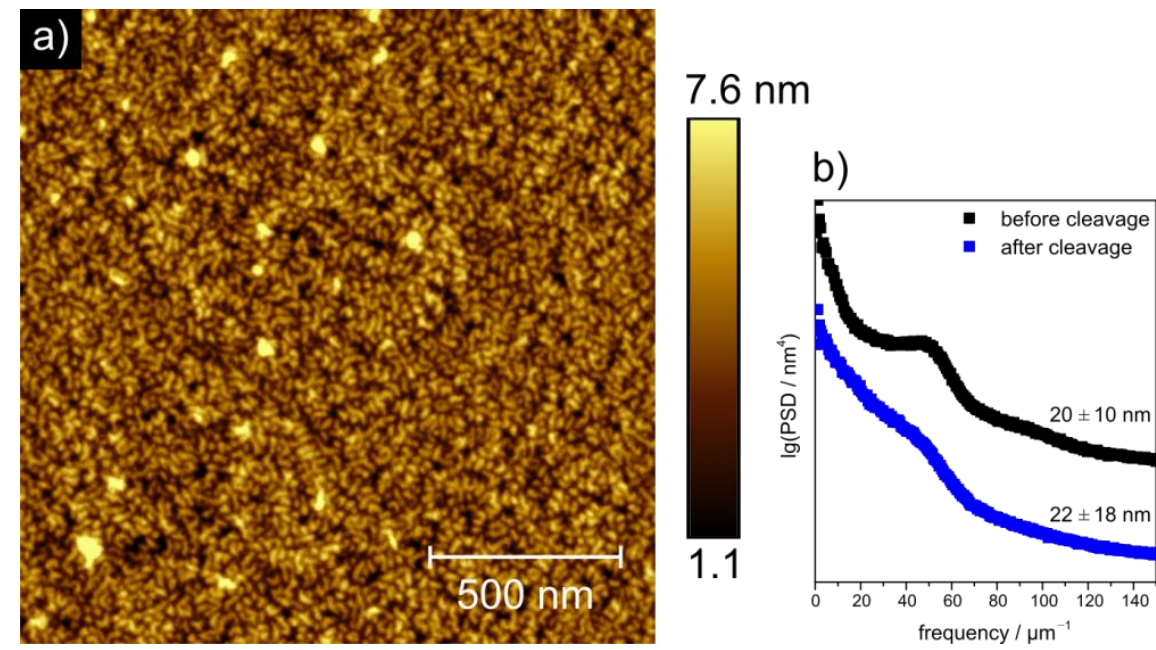

Figure 2.26. a) AFM topography image of cleavage product of sample III thermally annealed at $180{ }^{\circ} \mathrm{C}$ for 3 days under vacuum and b) two-dimensional, isotropic PSD distribution of the sample III prior to cleavage (black) and after cleavage (blue). The curves have been vertically shifted for clarity 


\subsection{2 $m$-(PS-b-PNIPAM) System}

Several studies have demonstrated that PS-PNIPAM systems are capable of forming microdomains. ${ }^{[99,114,133]}$ Different multiblock copolymer samples were synthesized using polyRAFT B. The multiblock copolymers vary in composition and average block number. Detailed information on the polymers is collected in Table 2.7. The polymer composition was determined via ${ }^{1} \mathrm{H}-\mathrm{NMR}$ spectroscopy as described in section 2.4. Using the densities $\rho_{\text {PS }}=1.05 \mathrm{~g} \cdot \mathrm{cm}^{-3}$ and $\rho_{\text {PNIPAM }}=1.1 \mathrm{~g} \cdot \mathrm{cm}^{-3}$ the volume fraction of PNIPAM $f_{\text {PNIPAM }}$ was determined. ${ }^{[99]}$ For the calculation of the average block number $\bar{b}$ and segments $\bar{s}$ only integer numbers of $N_{\text {TTC }}$ were used.

Table 2.7. Multiblock copolymer samples for microphase separation investigations with different PNIPAM comonomer composition $\phi_{\text {PNIPAM }}$ (determined via ${ }^{1} \mathrm{H}-\mathrm{NMR}$ spectroscopy) and volume fraction $f_{\mathrm{PNIPAM}}$ of PNIPAM. $\bar{M}_{\mathrm{n}, 0}$ denotes number average molar mass prior to cleavage of the multiblock copolymer and $\bar{M}_{\mathrm{n}, 1}$ after cleavage. For determination of the average number of blocks $\bar{b}$ and segments $\bar{s}$ equation (2.3) and (2.4) were employed.

\begin{tabular}{cccccccccc}
\hline Sample & $\begin{array}{c}\overline{\boldsymbol{M}}_{\mathrm{n}, \mathbf{0}} / \\
\mathbf{g} \cdot \mathbf{m o l}^{\mathbf{- 1}}\end{array}$ & $\boldsymbol{\Xi}$ & $\begin{array}{c}\overline{\boldsymbol{M}}_{\mathrm{n}, \mathbf{1}} / \\
\mathbf{g} \cdot \mathbf{m o l}^{\mathbf{- 1}}\end{array}$ & $\boldsymbol{\Xi}$ & $\boldsymbol{\phi}_{\text {PNIPAM }}$ & $\boldsymbol{f}_{\text {PNIPAM }}$ & $\boldsymbol{N}_{\text {TTC }}$ & $\overline{\boldsymbol{b}}$ & $\overline{\boldsymbol{s}}$ \\
\hline V & 128000 & 2.32 & 25200 & 1.90 & 0.78 & 0.77 & 4.0 & 5 & 9 \\
VI & 53100 & 2.28 & 15600 & 1.50 & 0.60 & 0.59 & 2.4 & 3 & 5 \\
VII & 61400 & 2.78 & 24200 & 1.63 & 0.74 & 0.73 & 1.5 & 3 & 5 \\
VIII & 72400 & 2.37 & 25800 & 2.00 & 0.78 & 0.77 & 1.8 & 3 & 5 \\
IX & 54600 & 1.46 & 13700 & 1.53 & 0.75 & 0.74 & 3.0 & 4 & 7 \\
X & 52400 & 1.77 & 34300 & 1.70 & 0.55 & 0.54 & 1.0 & 2 & 3 \\
\hline
\end{tabular}

Complementary to the AFM studies, DSC measurements were performed to obtain $T_{\mathrm{g}} \mathrm{s}$. The samples were measured in a temperature range from $30^{\circ} \mathrm{C}$ to $180{ }^{\circ} \mathrm{C}$. Two distinct $T_{\mathrm{gS}}$ for samples $\mathbf{V}, \mathbf{V I}, \mathbf{X}$ were observed which could be attributed to the $T_{\mathrm{gS}}$ of the two blocks (see Table 2.8). ${ }^{[123]}$ Figure 2.27 shows a representative DSC curve. The $T_{\mathrm{g}}$ around $100{ }^{\circ} \mathrm{C}$ results from the PS segments. For samples VII-IX no distinct $T_{\mathrm{g}} \mathrm{S}$ were observed. To explain the absence of the $T_{\mathrm{g}}$ in those samples the molar mass of PS has to be considered. The PS segments are too short to observe a $T_{\mathrm{g}}$. The second $T_{\mathrm{g}}$ at $140^{\circ} \mathrm{C}$ is assigned to the PNIPAM segments. ${ }^{[134]}$ It has to be mentioned that samples VI-IX were measured with a different DSC instrument. Therefore, the measured $T_{\mathrm{g}}$ are not directly comparable to each other. However, the measurements can provide the evidence of the existence of two $T_{\mathrm{g} s}$. Here, the observed $T_{\mathrm{g}}$ are also comparable to literature-known values of $\mathrm{AB}$ block copolymers. 


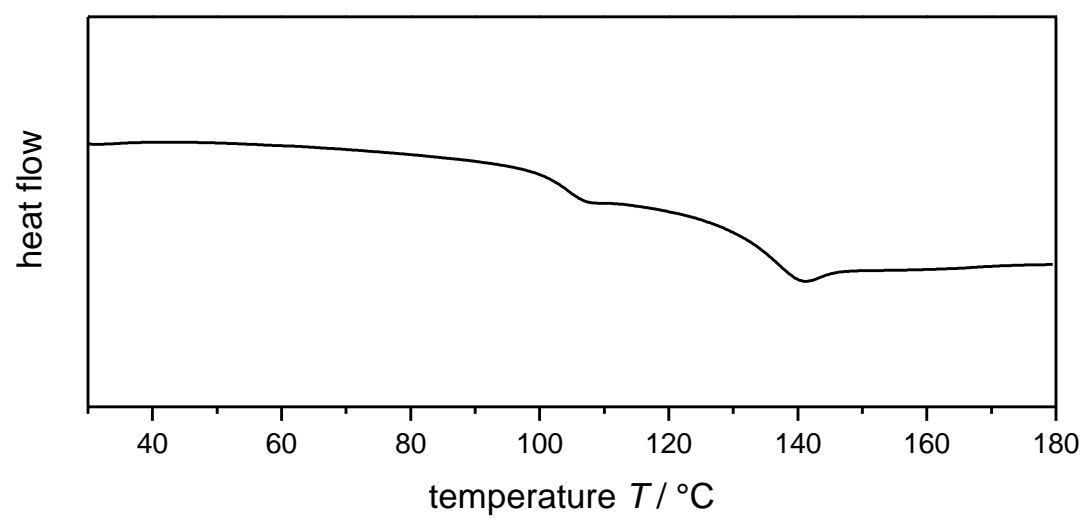

Figure 2.27. Representative DSC curve of multiblock copolymer $\mathbf{X}$.

Table 2.8. Glass transition temperatures $T_{\mathrm{g}}$ of $m$-(PS- $b$-PNIPAM) obtained from the DSC measurements and literature-known values for homopolymer of PS and PNIPAM.

\begin{tabular}{ccc}
\hline sample & $\boldsymbol{T}_{\mathrm{g}, 1} /{ }^{\circ} \mathrm{C}$ & $\boldsymbol{T}_{\mathrm{g}, 2} /{ }^{\circ} \mathrm{C}$ \\
\hline V & 97 & 140 \\
VI & 109 & 151 \\
VII & & 154 \\
VIII & & 156 \\
IX & & 158 \\
X & 103 & 135 \\
\hline
\end{tabular}

First, sample $\mathbf{V}$ was thermally annealed at $180^{\circ} \mathrm{C}$. Phase separation could not be observed. The respective image can be found in the Appendix A. Due to the lack of ordered structures no further samples were treated via thermal annealing. Instead, solvent annealing was used as method to obtain microdomain structures. In this method, the solvent vapor acts as a plasticizer on the block copolymer, reducing the $T_{\mathrm{g}}$ of at least one block below room temperature which results in an increase in chain mobility. One advantage is that solvent annealing is a faster method compared to thermal annealing. Structures can be obtained within minutes.[135] In addition, degradation of one or more blocks does not occur. Furthermore, unique morphologies may be accessible via solvent annealing inaccessible via thermal annealing. ${ }^{[108,136]}$ 


\subsubsection{Microphase Separation using Pure Solvents and Solvent Mixtures}

Several studies have shown the morphology can be tailored by the use of selective solvents. ${ }^{[99,137]}$ Here, common laboratory solvents were selected such as THF, methanol (MeOH) and toluene (Tol). To compare the selectivity of these solvents for the blocks in the multiblock copolymer the interaction parameters were calculated. The solvent-polymer interactions can be estimated by the Hildebrand solubility parameters.[119,137,138] The interaction parameter $\chi_{\mathrm{H}}^{\infty}$ (enthalpy related and infinite dilution) can be calculated using the following equation:

$$
\chi_{\mathrm{H}}^{\infty}=\frac{V_{\mathrm{i}}}{\mathrm{RT}}\left(\delta_{\mathrm{i}}-\delta_{\mathrm{j}}\right)^{2}
$$

Herein, $V_{\mathrm{i}}$ is the average molar volume of the solvent, $\delta_{\mathrm{i}}$ and $\delta_{\mathrm{j}}$ are the solubility parameters of the solvent and polymer, respectively. $\mathrm{R}$ is the ideal gas constant and $T$ is the absolute temperature. The values of $V_{\mathrm{i}}$ and $\delta$ are calculated according to van Krevelen using group contributions and listed in Table 2.9. ${ }^{[139]}$ Unfortunately, no solubility parameters are known for the copolymer of PS-PNIPAM.

Table 2.9. Average molar volume $V_{\mathrm{i}}$ and calculated Hildebrand solubility parameter $\delta .[132]$

\begin{tabular}{|c|c|c|}
\hline & Solubility parameter $\delta /(\mathrm{MPa})^{1 / 2}$ & Molar volume $/ \mathrm{cm}^{3} / \mathrm{mol}$ \\
\hline THF & 18.6 & 81.9 \\
\hline Tol & 18.2 & 106.6 \\
\hline $\mathrm{MeOH}$ & 29.7 & 40.6 \\
\hline PS & 18.6 & \\
\hline PNIPAM & 23.5 & \\
\hline
\end{tabular}

In general, the interaction parameter should be $\chi_{\mathrm{H}}^{\infty} \leq 0.5$ or the differences in solubility parameters should not be greater than $5(\mathrm{MPa})^{1 / 2}$ for effective solubility or swelling the polymer in a particular solvent. [98,137] Therefore, methanol can be considered as PNIPAM-selective solvent. THF and toluene are selective for the PS block.[99,140]

Table 2.10. Calculated $\chi_{\mathrm{H}}^{\infty}$ parameters using equation (2.9).

\begin{tabular}{llccc}
\hline & & MeOH & THF & Tol \\
\hline$\chi_{\mathrm{H}}^{\infty}$ parameters & PS & 2.02 & 0 & 0 \\
& PNIPAM & 0.69 & 0.79 & 1.21 \\
\hline
\end{tabular}


First, a high molar mass (128000 g.mol-1) multiblock copolymer sample $\mathbf{V}$ was synthesized to ensure a high segregation product $\chi N$. It exhibits on average 5 blocks and 9 segments with a rather high overall dispersity of 2.2. A thin polymer film was prepared via spin coating the multiblock copolymer $(2 \mathrm{wt} \%$ in THF) on a silicon wafer substrate. The effect of different solvents on the morphology was investigated. For solvent annealing the substrate was placed inside a solvent chamber, which was first evacuated and then exposed to the solvent vapor. The solvent annealing process was stopped by fast quenching which was executed by evacuating the solvent chamber to remove all solvent from the chamber. Due to this technique changes in the morphology upon drying should be prevented and the morphology will be kinetically trapped. $[99,101,141]$

Pure solvents were used for solvent annealing of sample V. According to the AFM image in Figure 2.28 the as-spun thin film showed a disordered structure. For solvent annealing using toluene, a perforated lamellar structure was observed. Toluene is a PS-selective solvent which swells the polystyrene blocks selectively and shifts the volume fraction to higher PS content. Thus, a shift within the phase diagram to another region is accessible without changing the molar mass or the comonomer composition of PNIPAM. The THF-annealed films exhibited a smooth surface with some unorganized darker dots which indicate no phase separation. However, the methanol-annealed films showed light dots within a darker matrix. Considering the volume fraction of PNIPAM $\left(f_{\text {PNIPAM }}=0.78\right)$ the light dots can be assigned to PS blocks and the matrix to PNIPAM. This structure can be attributed to hexagonally packed cylinder morphology perpendicular to the substrate. 


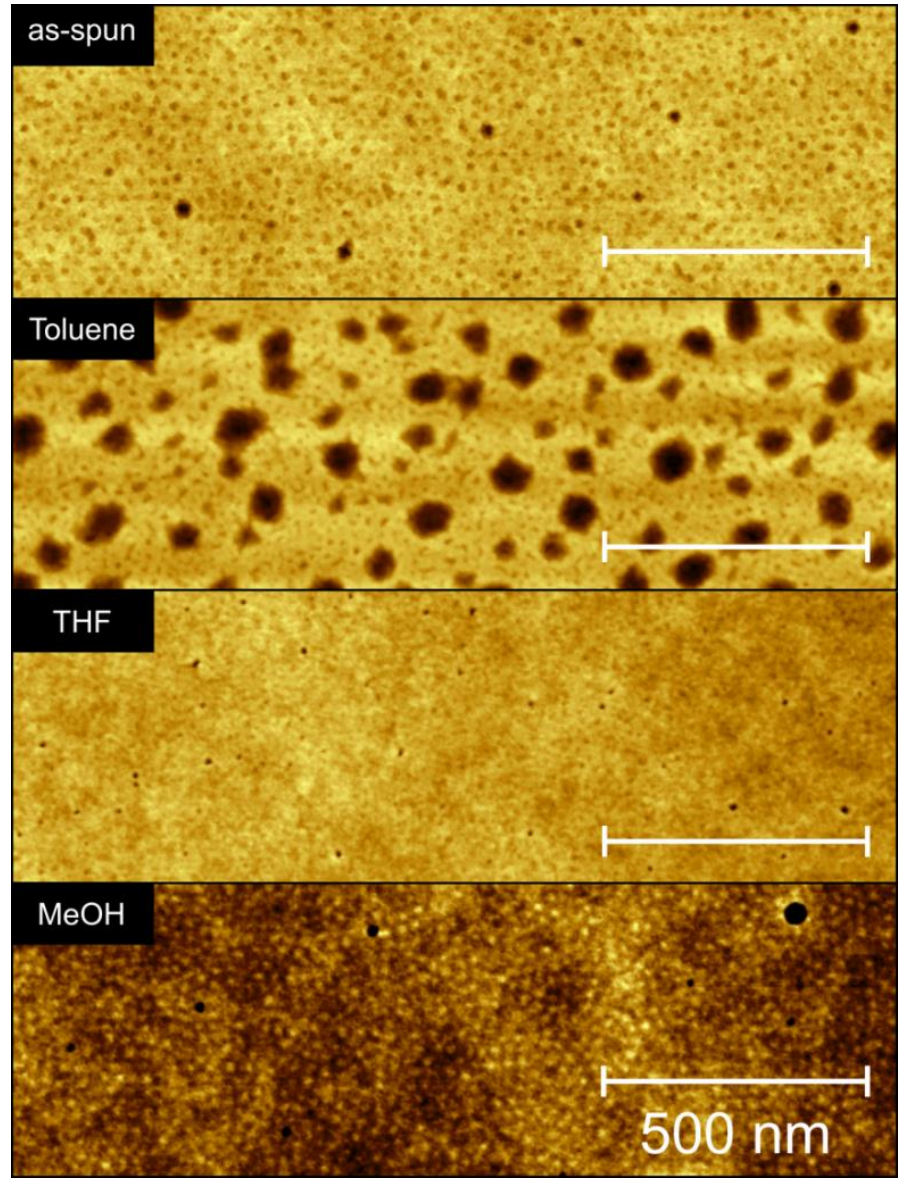

Figure 2.28. AFM topography images of sample $\mathbf{V}$ solvent dependent annealing for $2 \mathrm{~h}$.

Besides pure solvents, selective solvent mixtures were applied. It was expected that the morphologies would differ from the pure solvents. Figure 2.29 shows the AFM images of the annealed thin films using methanol-THF and methanol-toluene mixtures. Using the solvent mixture methanol-THF 1:2 (v:v) no phase separation was observed. Hexagonal cylinders perpendicularly orientated to the substrate were obtained by reducing the amount of THF compared to methanol. Considering the volume fraction of PNIPAM $f_{\text {PNIPAM }}=0.78$ the cylinders can be assigned to PS segments and the matrix to PNIPAM. By increasing the methanol-THF ratio from 1:1 to 3:1 (v:v) the cylinders were swollen. As a result, a certain volume of PNIPAMselective methanol is necessary in the solvent mixture to form hexagonal cylinders. For annealing using methanol-toluene solvent mixture, 
perpendicular cylinders are also formed. As mentioned above, the volume ratio was varied from $1: 2$ to $3: 1(\mathrm{v}: \mathrm{v})$ for methanol-THF mixture and 1:1 to 2:1 (v:v) for methanol-toluene. By adjusting the solvent ratio to the composition of the multiblock copolymer the microdomain formation is promoted.

The cylinder-to-cylinder $D_{0}$ distance was determined form the maxima in the PSD (see Figure 2.30). The distance increases with increasing amount of methanol in the solvent mixture, as shown in Table 2.11. For methanolTHF mixtures (v:v), the distance expands from $23 \mathrm{~nm}(1: 1)$ over $26 \mathrm{~nm}(2: 1)$. The effect is even stronger for the methanol-toluene mixture. This might be to the fact, that both solvents are selective for the particular blocks. The cylinder-to-cylinder distance increases from $25 \mathrm{~nm}$ (2:1) up to $30 \mathrm{~nm}$ (3:1).

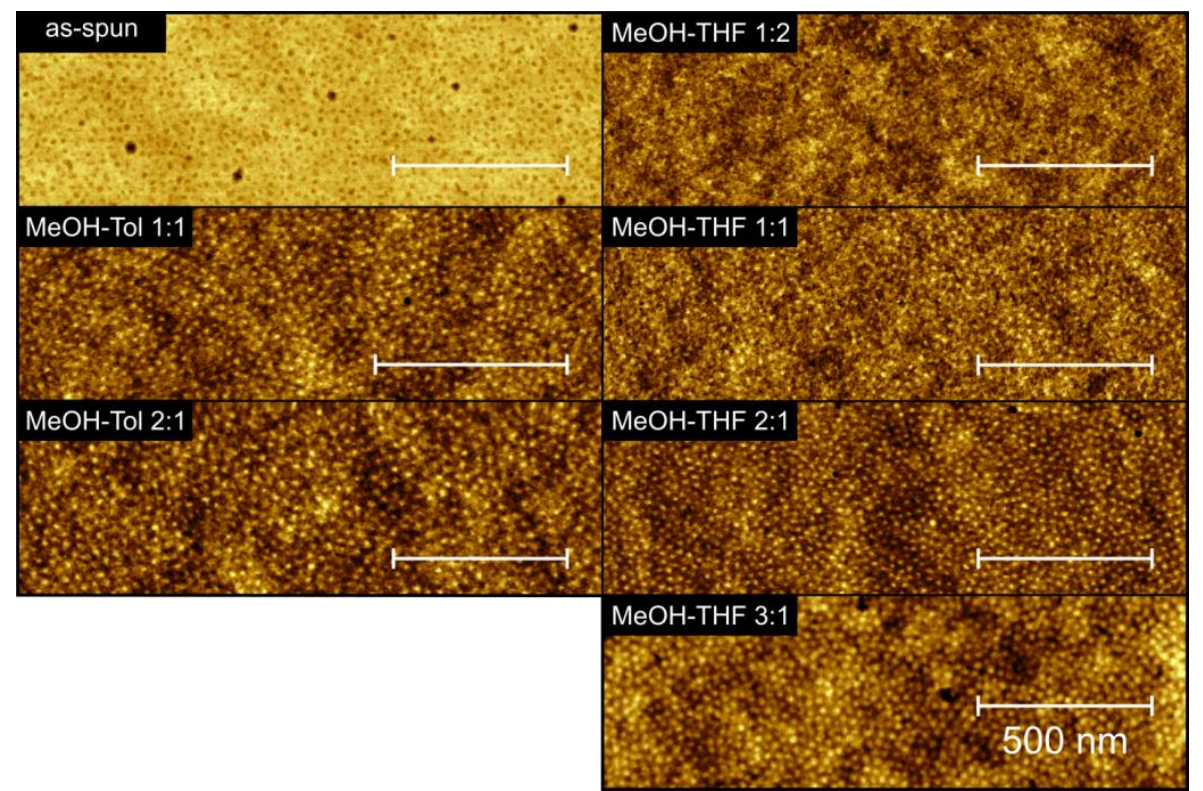

Figure 2.29. AFM topography images of sample $\mathbf{V}$ after solvent annealing using methanol-THF and methanol-toluene mixtures (v:v).

Table 2.11. Cylinder-to-cylinder distance $D_{0}$ determined by baseline correction and following Lorentzian fit of the PSD-distribution shown in Figure 2.30.

\begin{tabular}{ccc}
\hline solvent mixtures & (v:v) & $\mathbf{D}_{\mathbf{0}} / \mathbf{n m}$ \\
\hline \multirow{2}{*}{ MeOH-Tol } & $1: 1$ & $25 \pm 5$ \\
& $2: 1$ & $30 \pm 9$ \\
\hline & $1: 1$ & $23 \pm 9$ \\
MeOH-THF & $2: 1$ & $26 \pm 7$ \\
& $3: 1$ & $26 \pm 7$ \\
\hline
\end{tabular}



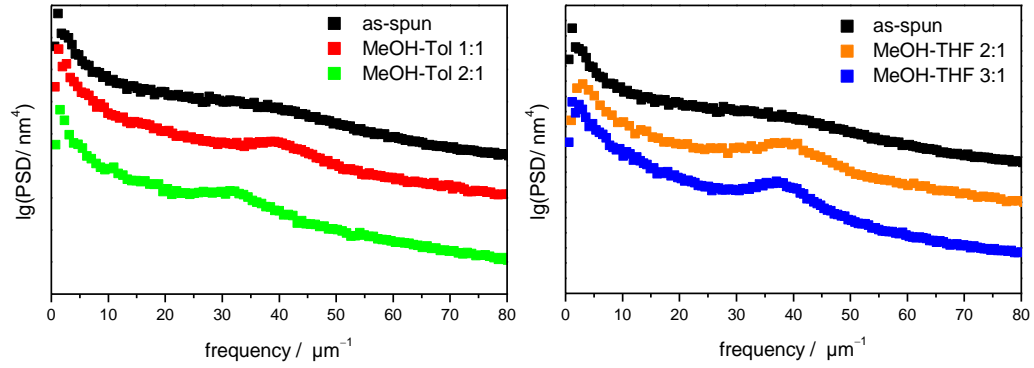

Figure 2.30. Two-dimensional, isotropic PSD-distribution of the height profiles shown in Figure 2.29. The curves have been vertically shifted for clarity.

Figure 2.31 shows the effect of the selectivity of the solvents. Toluene changes the volume fraction upon swelling leading to perforated lamellar. However, solvent mixtures such as methanol-THF and methanol-toluene result in nicely ordered hexagonally cylinders perpendicular to the substrate.

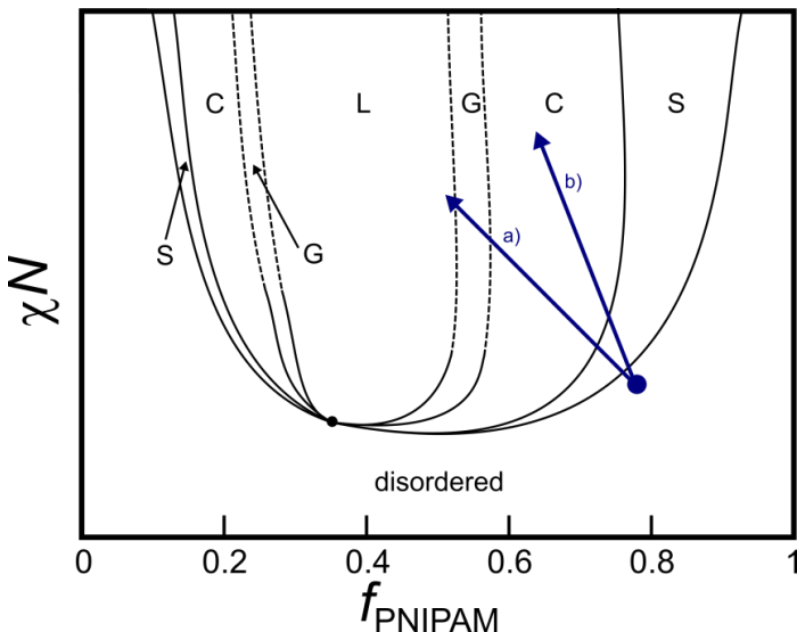

Figure 2.31. Possible trajectory of the multiblock copolymer $\mathbf{V}$ in the previous introduced phase diagram (see section 2.3) upon swelling with a) toluene, b) solvent mixture MeOH-THF and MeOH-Tol 2:1 (v:v). 


\subsubsection{Influence of the Film Thickness and Time on the Microstructure}

As described above, the sample $\mathbf{V}$ is capable of undergoing microphase separation in various solvent mixtures. The film thickness is a crucial factor for the formation of microdomains. Several studies have shown that morphology depends on the film thickness.[102,119,142-144] In general, by increasing the film thickness perpendicular orientation of the cylinders might get lost. In theory, neutral interfaces (substrate and surface/air) maintain the perpendicular orientation of the cylinders over a wide range of thicknesses. [143-145]

Here, different solvent casting methods, solvent casting and spin coating, were used to achieve a variation of the film thickness. First, a polymer solution ( $2 \mathrm{wt} \%$ in THF) was prepared. Afterwards, a specific amount of the solution was either drop casted or spin coated onto a silicon wafer substrate. The solvent casted film was left until the solvent evaporated. In addition, the spinning velocity for the spin coating was varied from $2500 \mathrm{rpm}$ to $3500 \mathrm{rpm}$. Then the polymer films were annealed using methanol-THF 2:1 (v:v) for $2 \mathrm{~h}$. The film thickness was analyzed by ellipsometry which revealed a rather thick film for solvent casting $(72 \pm 10) \mu \mathrm{m}$. For the spin coating films, thicknesses of $(148.1 \pm 0.4) \mathrm{nm}$, $3500 \mathrm{rpm}$, and $(174.8 \pm 0.4) \mathrm{nm}, 2500 \mathrm{rpm}$, were produced. The thickness of a film decreases appropriately with increasing square-root of the spin velocity which is in good agreement with the experimental data. Figure 2.32 shows the observed morphologies upon annealing. The drop casting film showed weak formation of cylinders. However, the thin films of spin coating showed a better self-assembled structure. Furthermore, the cylinder-to-cylinder distance $D_{0}$ changes for higher spinning speed. The distance was determined via the maximum peak of the PSD distribution of the height profiles (see Figure 2.33). The drop-casted film exhibited no clear maximum, thus no cylinder-to-cylinder distance was specified. A slightly larger distance was found for spin coating at a velocity of $2500 \mathrm{rpm}$, $(28 \pm 9) \mathrm{nm}$ than at $3500 \mathrm{rpm},(26 \pm 7) \mathrm{nm}$. By increasing the film thickness multiple layers of the multiblock copolymer were formed. Therefore, the larger amount of material arranges slower than thinner films into the microdomains.[146] To conclude, the formation of microphase structures is promoted in thinner films. 


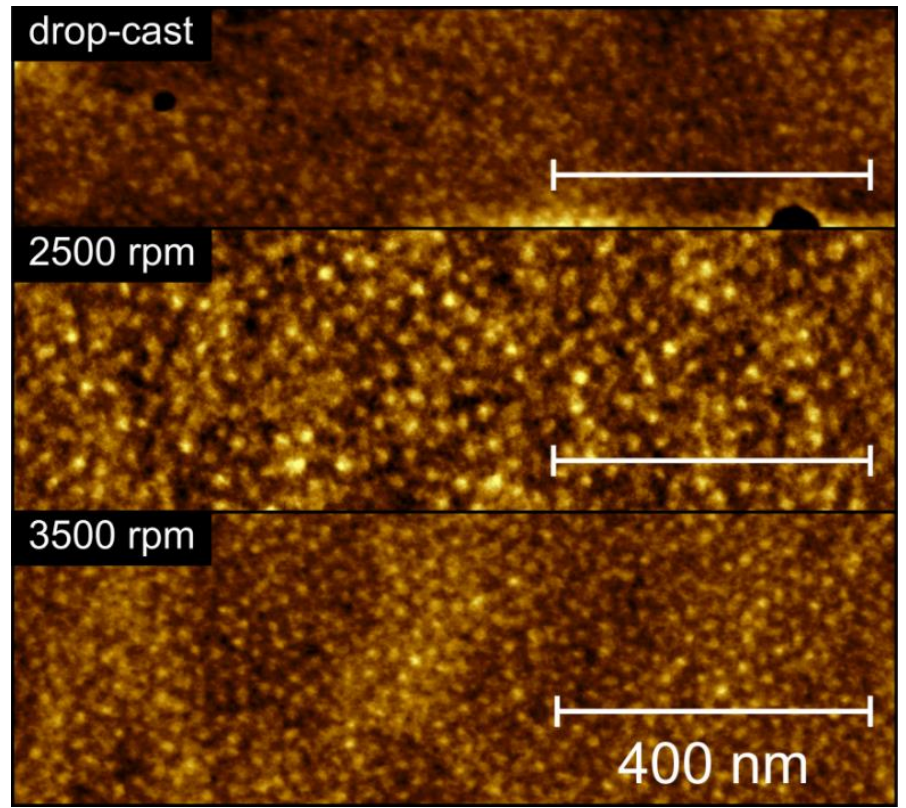

Figure 2.32. Influence of the casting-technique and spinning rate on the morphology of the sample $\mathbf{V}$.

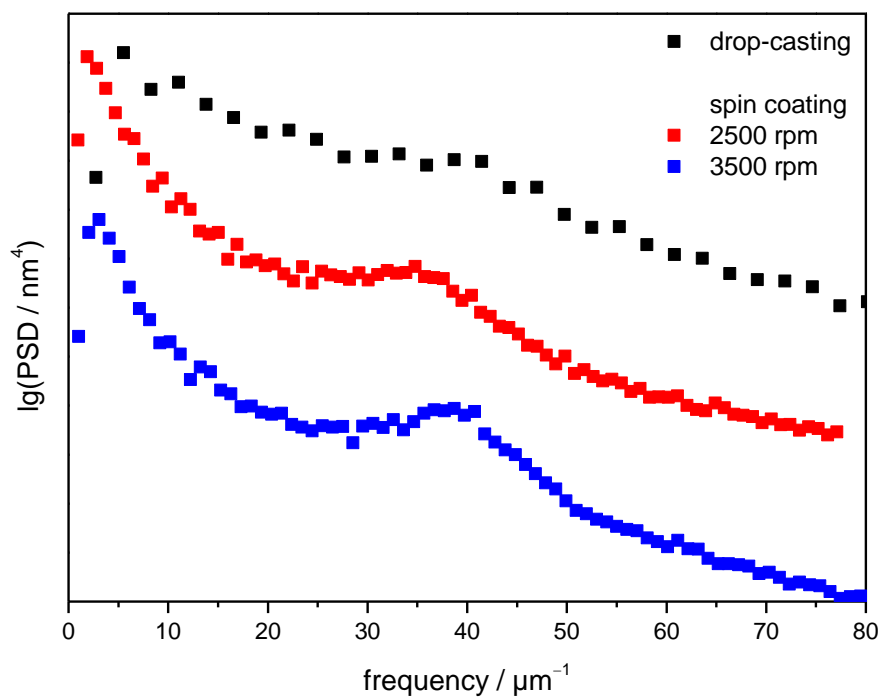

Figure 2.33. Two-dimensional PSD distribution of the AFM height profile shown in Figure 2.32. The curves have been vertically shifted for clarity. 
The formation of microstructures also depends on the annealing time. Several studies have shown that the morphology changes with varying the annealing time. ${ }^{[99,135,147]}$ Thin films of sample $\mathbf{V}$ were solvent annealed using methanol-THF 2:1 (v:v) at different annealing times. Figure 2.34 shows that the self-assembled microdomains could be observed after one hour. Welldefined hexagonally packed cylinders perpendicular to the substrate were formed. By increasing the annealing time, no change in the orientation of cylinders was observed. Furthermore, the cylinder-to-cylinder distance did not change upon increasing the annealing time. Therefore, well-organized cylinders are generated after a short annealing time.

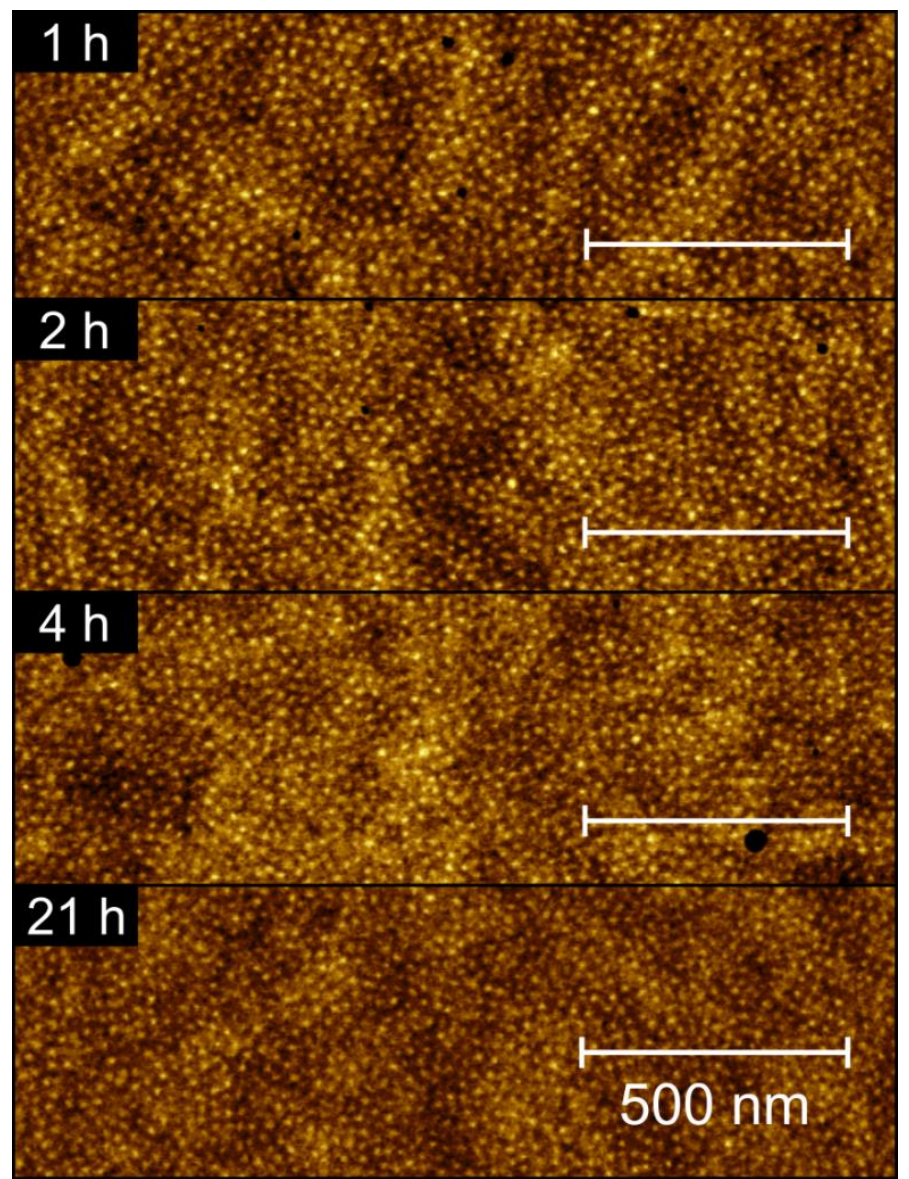

Figure 2.34. AFM topography images of sample $\mathbf{V}$ of time dependent solvent annealing using MeOH-THF 2:1 (v:v). 


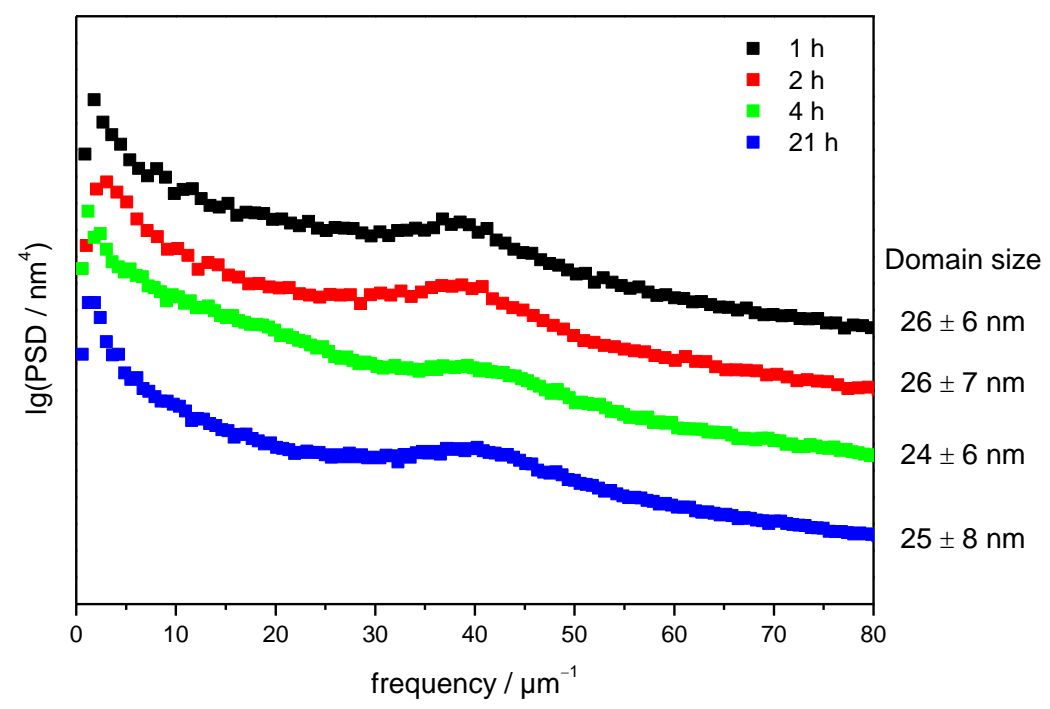

Figure 2.35. Two-dimensional PSD distribution of the AFM height profile shown in Figure 2.34. The curves have been vertically shifted for clarity.

\subsubsection{Influence of the Volume Fraction on the Microphase Separation}

So far, the capability to undergo microphase separation of the PS-PNIPAM system was examined exemplarily with sample V. Sample V has a rather high molar mass which should ensure a high value of $\chi N$ to observe microphase separation. For the following investigations multiblock copolymers with a constant average block number of 3 were prepared. Therefore, multiblock copolymers with a constant PS segment length and increasing PNIPAM fraction were synthesized (sample VI-VIII). The detailed information is summarized in Table 2.7. The thin films were prepared as described above and annealed using methanol-THF 2:1 (v:v) for $2 \mathrm{~h}$. Figure 2.36 shows the resulting AFM topography image of VI. For better visualization, the color scale is changed from a golden to spectral gradient. On the one hand, large dots (green-blue) randomly distributed onto the surface are observed which display inhomogeneity of the thin film. Impurities from the polyfunctional RAFT agent in the polymer can cause this inhomogeneity of the thin film. On the other hand, smaller light dots and worm-like objects within a darker matrix are visible. The sample 
exhibits a mixed morphology of perpendicular cylinders and either parallel cylinders or perpendicular lamellae of PS in a PNIPAM matrix.
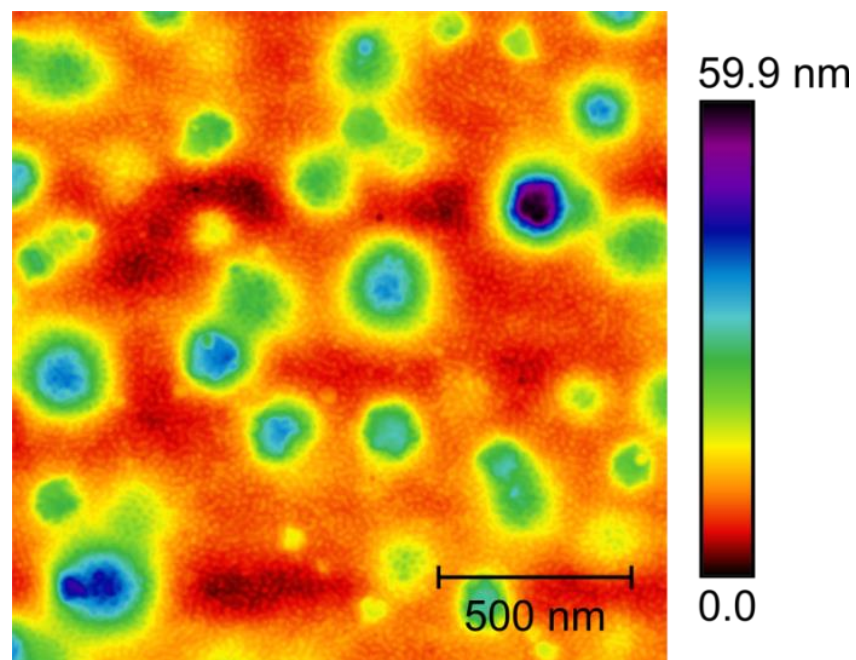

Figure 2.36. AFM topography image of multiblock copolymer sample VI with $\phi_{\text {PNIPAM }}=0.60$, after solvent annealing using MeOH-THF 2:1 (v:v) for $2 \mathrm{~h}$.

By increasing the volume fraction of PNIPAM to $f_{\text {PNIPAM }}=0.73$ only perpendicular cylinders are visible, similar to sample V. Further increasing the volume fraction to $f_{\text {PNIPAM }}=0.77$ did not change the morphology (see Table 2.12). Figure 2.37 shows the corresponding AFM images. It was demonstrated that a microphase separation is already visible with overall molar mass of $53000 \mathrm{~g} \cdot \mathrm{mol}^{-1}$ and average molar mass for the cleaved blocks of $15600 \mathrm{~g} \cdot \mathrm{mol}^{-1}$. Here, it has to be mentioned that the characterization of the cleavage products by SEC did not provide the possibility to distinguish between end and middle blocks. In literature, only phase separation for $A B$ block copolymers of PS-PNIPAM starting with a molar mass of $20000 \mathrm{~g} \cdot \mathrm{mol}^{-1}$ and $f_{\text {PNIPAM }}=0.68$ is $\mathrm{known}$. ${ }^{133]}$ 

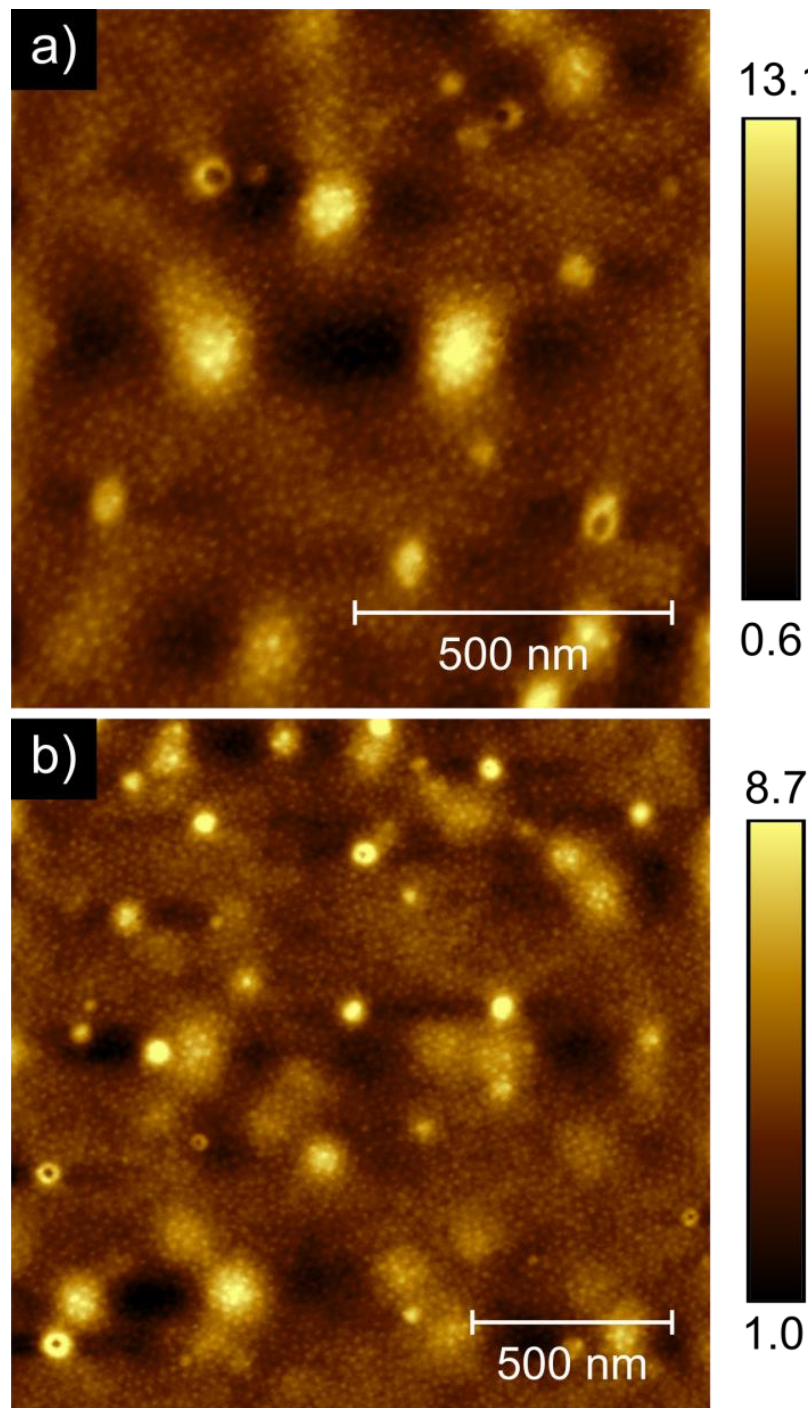

Figure 2.37. AFM topography images of multiblock copolymer sample a) VII and b) VIII after solvent annealing using MeOH-THF 2:1 (v:v) for $2 \mathrm{~h}$.

Table 2.12. Morphologies of the synthesized $m$-(PS- $b$-PNIPAM) with varied volume fraction of PNIPAM. The described orientation is relative to the substrate.

\begin{tabular}{ccc}
\hline sample & $f_{\text {PNIPAM }}$ & morphology \\
\hline VI & 0.59 & perpendicular and parallel cylinders \\
VII & 0.73 & perpendicular cylinders \\
VIII & 0.77 & perpendicular cylinders \\
\hline
\end{tabular}




\subsubsection{Cleavage Products of Multiblock Copolymers and Their Phase Separation Behavior}

In theory, multiblock copolymers and their individual blocks are expected to show the same morphology.[29] To prove this fact, multiblock copolymers V-IX were cleaved by an excess of radicals in order to split them. The cleavage results in $\mathrm{AB}$ and $\mathrm{ABA}$ block copolymers due to the different block species which are present in multiblock copolymers (see section 2.2.2). The cleavage products were spin coated, solvent annealed using methanol-THF 2:1 (v:v) and examined via AFM. Figure 2.38 shows the corresponding AFM image. For comparison, the phase separation images of the corresponding multiblock copolymers are also displayed. In Figure $2.38 \mathrm{~b}$, the solvent annealed cleaved product of sample $\mathbf{V}$ is present. A rather smooth surface is visible and no ordered structure is observed. The lack of microphase separation could be explained by a lower effective segregation product $\chi N$ of the block copolymers. It consists of the product of the degree of polymerization $N$ and the interaction parameter $\chi$. The individual blocks exhibit an average molar mass of $25200 \mathrm{~g} \cdot \mathrm{mol}^{-1}$. The $\chi N$ value gets too low to observe phase separation due to the low molar mass and degree of polymerization $N$. However, the cleavage products of multiblock copolymer VI-VIII showed self-assembled structures which exclude too low a $\chi N$ for the cleavage products of sample $\mathbf{V}$. The samples were dissected with an excess of radicals using AIBN as radical source. Since the cleaved polymers are used without any further purification, it cannot be ruled out that the by-products of the cleavage reaction influence the capability of the microphase separation. After dissecting the multiblock copolymer VI the previous mixed morphology disappeared and lying cylinders or perpendicular lamellar are obtained. By increasing the PNIPAM content of the polymer (see Figure 2.38f and Figure 2.38h) perpendicular cylinders are visible after cleavage.

The reason for those rather contradictory results is still not entirely clear. For more detailed investigation of those opposing results, a multiblock copolymer (sample IX) exhibiting a comparable overall molar mass to sample VI was prepared. The solvent annealed morphologies before and after cleavage are displayed in Figure $2.38 \mathrm{i}$ and $\mathrm{j}$, respectively. Before cleavage, the multiblock copolymer IX possesses well-arranged hexagonally packed cylinders of PS within a PNIPAM matrix. After cleavage, no ordered structure can be obtained which is in contrast to sample VI. The thin films differ in the PNIPAM comonomer composition. This fact should not influence the degree of incompatibility because the overall degree of polymerization is used to determined $\chi N$. As mentioned above, it is very likely that the by-products of the cleavage reaction have an 
influence on the microphase separation behavior. Another crucial factor to consider is that the morphology depends on the film thickness.[119,142] Therefore, the thickness of the films was kept approximately the same for all samples, monitored by ellipsometry. Another explanation and the most reasonable point, the cleavage of the multiblock copolymers results in $\mathrm{AB}$ and ABA species (see section 2.2.2). The ABA species might exhibit twice the chain length of the $A B$ species. SEC characterization of the cleaved polymers did not show the existence of distinct species. Nevertheless, two different species of polymers are present in the cleavage product. Upon closer examination it seems that if the number of ABA polymers exceeds the number of $\mathrm{AB}$ polymers the phase separation is not present.

The present study has only investigated a few samples over a small range of volume fraction and block numbers. Further work needs to be done to establish whether the microphase separation is hampered by the ratio of $\mathrm{AB}$ to $\mathrm{ABA}$ block copolymers. To avoid such cleavage caused effects, $\mathrm{AB}$ block copolymers comparable to the individual blocks should be synthesized. Despite this observation, highly ordered structures are obtained for the multiblock copolymer in short amount of time. 


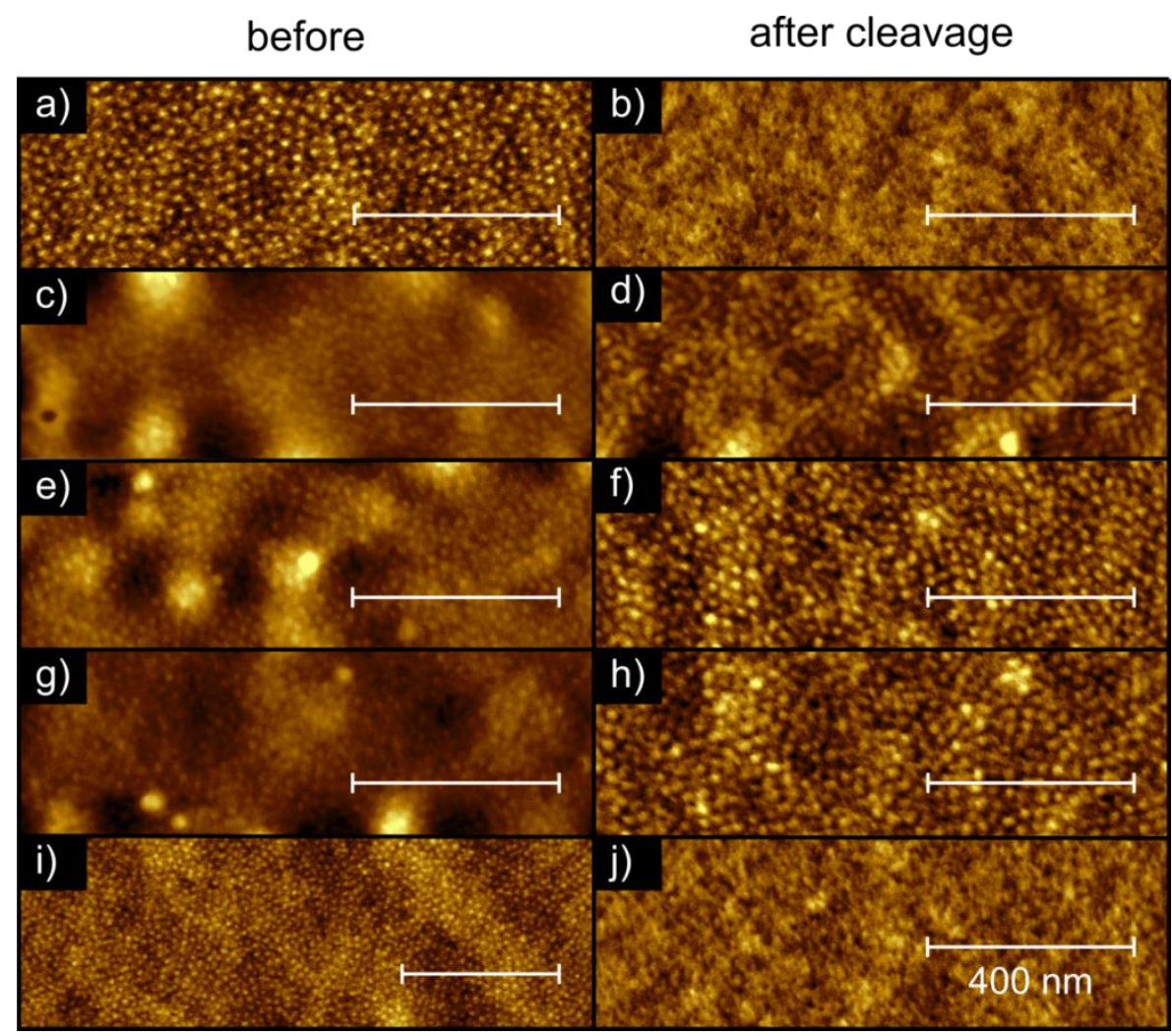

Figure 2.38. AFM topography images after solvent annealing using MeOH-THF 2:1 (v:v) for $2 \mathrm{~h}$ of multiblock copolymer a) V, c) VI, e) VII, g) VIII and i) IX and the corresponding cleavage products of the multiblock copolymers b) V, d) VI, f) VII, h) VIII and j) IX.

\subsubsection{PS-b-PNIPAM- $b$-PS Block Copolymer}

So far multiblock copolymers possessing block numbers greater than 2 were investigated. Here, the block copolymer $\mathbf{X}$ containing 2 blocks (three segments) was synthesized by using polyRAFT A (for detailed polymerization conditions see Table 6.6) and the microphase separation behavior was investigated in detail. In literature, ABA block copolymers are mostly assigned as triblock copolymer. In this section, this term will also be used despite the definition in the beginning (see section 2.2.2). Thin polymer films were prepared via spin coating the multiblock copolymers ( $2 \mathrm{wt} \%$ in THF) onto a silicon wafer substrate. Afterwards, the films were investigated on their microphase separation behavior by using pure solvent and solvent mixtures. Figure 2.39 shows the resulting AFM images. It has to 
be mentioned that the height scales of some of the images were not aligned to zero in order to keep a high color contrast of the structures. The as-spun film displays a micelle-like structure with no orientation alignment. This morphology can be described as non-organized perpendicular cylinders. After solvent annealing with THF or toluene no difference of film microstructure was observed compared to the as-spun film. Changing the solvent to methanol resulted in a different morphology. A mesh-like structure was observed. Due to the selectivity of methanol, the PNIPAM blocks becomes swollen and alters the preferred orientation of the cylinders from perpendicular to parallel.[99] The large dark holes in the image are defects of the film caused by contamination of the substrate prior to use.

Besides the pure solvents, solvent mixtures of methanol and toluene or THF were used for solvent annealing. A volume-to-volume ratio of 1:1 for the solvent mixture was selected since the polymer has a volume fraction of $f_{\text {PNIPAM }}=0.54$. Earlier investigations showed that the structure is pronounced by increasing the amount of the selective solvent in mixtures (see section 2.5.2.1). Surprisingly, both films showed a similar selfassembled structure. The introduction of THF or toluene to the PNIPAM-selective methanol led to either parallel to the substrate orientated cylinders or perpendicular lamellae. The strain energy of the film is lowered and such morphologies can be observed. Of noteworthy mention is that film thickness is a crucial factor in the orientation of the domains. It is known that in thin films the elastic strain energy associated with forming a parallel alignment is too large unless an integer number of layers of cylinders are formed and instead perpendicular alignment is favored. Commonly, the perpendicular arrangement of cylinders or lamellae is favored. ${ }^{[98]}$ In this work, the film thickness was $(143 \pm 1) \mathrm{nm}$ for all films of sample $\mathbf{X}$ to avoid such thickness effects. A definite conclusion regarding the morphology present cannot be determined without detailed measurements like SAXS.[148] Furthermore, theoretical simulations showed that weak attractions to the substrate result in non-vertical alignment of cylinders rather than unordered parallel cylinders which could be possible in those morphologies. ${ }^{[149]}$ The domain size was determined via the maximum peak of the PSD function (see Table 2.13). The baseline was corrected and the position of the maximum was fitted by a Lorentzian fit. The domain size increased for annealing using toluene as well for THF due to the increased mobility of PS segments. By using solvent mixture the domain size is also increased compared to the as-spun films. 

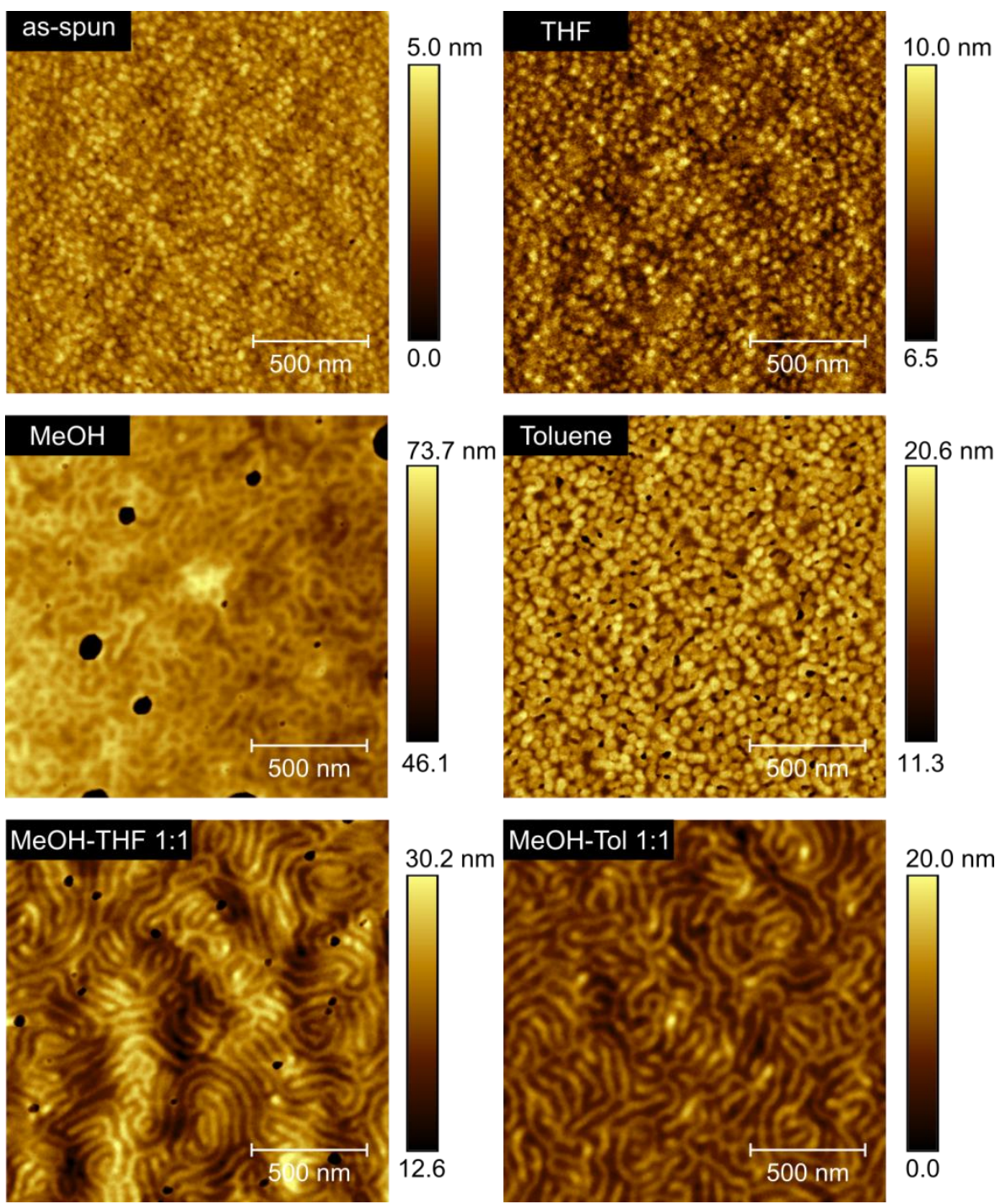

$20.0 \mathrm{~nm}$

Figure 2.39. AFM topography images of sample $\mathbf{X}$ as-spun and after solvent annealing with pure solvents and solvent mixtures after $2 \mathrm{~h}$. 
Table 2.13. Domain size determined via baseline correction and following Lorentzian fit of the PSD function of the height image of Figure 2.39.

\begin{tabular}{cc}
\hline solvent & domain size / nm \\
\hline as-spun & $44 \pm 8$ \\
Tol & $56 \pm 6$ \\
MeOH & - \\
THF & $48 \pm 7$ \\
MeOH-THF 1:1 & $54 \pm 5$ \\
MeOH-Tol 1:1 & $66 \pm 8$ \\
\hline
\end{tabular}

The local characterization of the Young's modulus by the DerjaguinMuller-Toporov (DMT) model and adhesion profiles allows the assignment of the domains. Therefore, AFM images were obtained using the PeakForce Quantitative Nano-Mechanics (PFQNM) imaging mode. It has to be mentioned that the scale bar for those images is a relative scale since no appropriate calibration for the measurement was available. Figure 2.40 shows a set of AFM images of the solvent annealed sample $\mathbf{X}$ using methanol-toluene 1:1 (v:v). The adhesion image (Figure 2.40b) displays light areas as high adhesiveness and dark areas as lower adhesiveness. Since PNIPAM has a higher adhesion force than PS, the lying cylinder objects can be assigned to PS segments and the matrix to PNIPAM.[131,150] For clarity, one position of the PS phase is marked white in the AFM images (see Figure 2.40). In addition, the logarithmic DMT profile is shown in Figure 2.40c. The light areas correspond to a stiff material and darker areas to a softer matter. The assignment of the blocks can be confirmed by the Young's modulus. PS exhibits a higher Young's modulus (2.5 GPa) compared to PNIPAM (0.7 GPa).[131,150] 

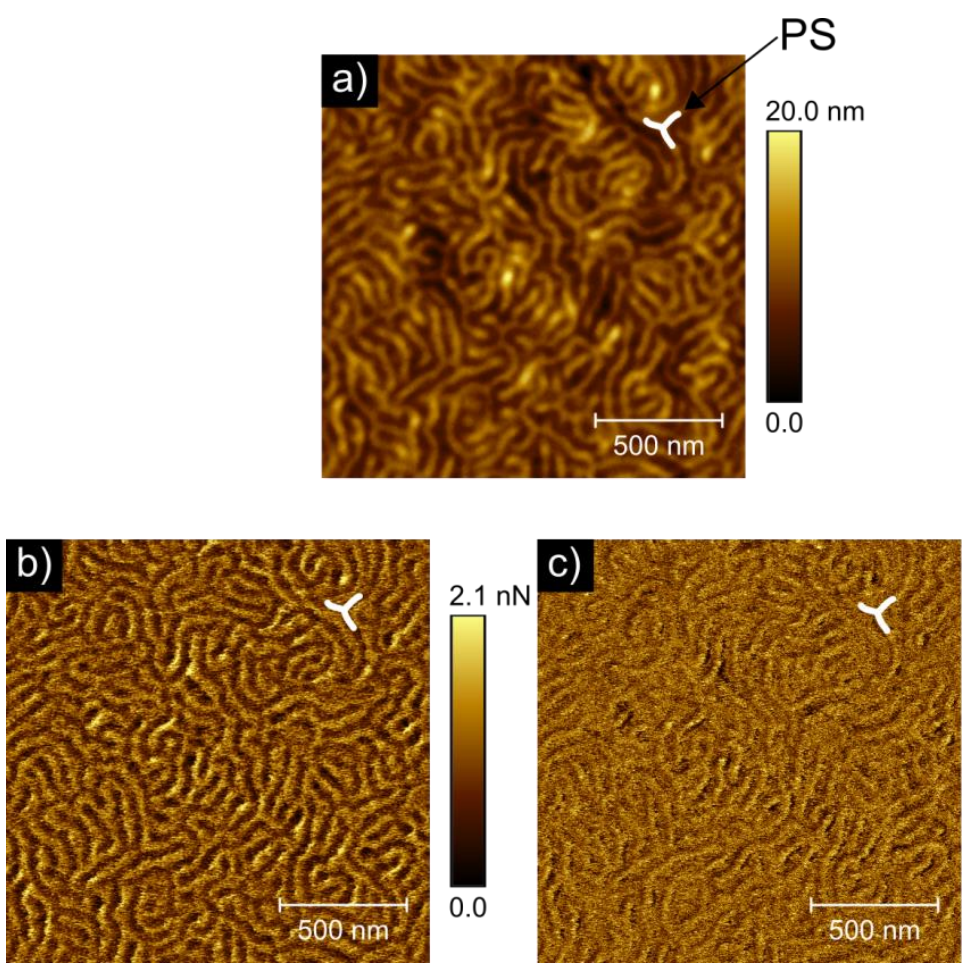

$71.8 \mathrm{mlog}(\mathrm{MPa})$

Figure 2.40. AFM images of sample $\mathbf{X}$ solvent annealing using MeOH-Tol 1:1 (v:v) a) topography, b) adhesion and c) $\log$ DMT modulus images with an exemplary marked PS phase.

\section{Combination of Solvent and Thermal Annealing}

The influence of temperature on the pre-solvent annealed film was investigated. Studies have shown that the combination of solvent and thermal annealing can improve the observed microstructure. ${ }^{[106]}$ The sample annealed with methanol-toluene 1:1 (v:v) was placed in a vacuum oven and thermally annealed at temperatures above $100{ }^{\circ} \mathrm{C}$ over night. Afterwards, the solvent-thermal annealed film was analyzed via AFM. Figure 2.41 shows the corresponding AFM images after thermal treatment at different temperatures. The size of the light domain was increased. Chain stretching is enhanced with increasing temperature and due to this the domain size increases. After annealing at $120^{\circ} \mathrm{C}$ the structure is inverted. The previous light areas are converted into dark areas. First, it was suspected that remaining solvent from the solvent annealing was enclosed into the microstructure which was then removed at higher temperature. To exclude this hypothesis, measurements were performed by ellipsometry to 
determine the residual solvent content inside the film. The content was obtained by modeling the refractive index with a three-medium Brüggeman model system resulting in less than $1 \%$ of solvent remaining inside the structure. Another explanation for the reorganization of the structure is that the surface energy of the blocks changes with temperature. At room temperature, PS $\left(\gamma_{\mathrm{PS}}=41 \mathrm{mN} \cdot \mathrm{m}^{-1}\right)$ has a higher surface energy compared to PNIPAM $\left(\gamma_{\text {PNIPAM }}=38.9 \mathrm{mN} \cdot \mathrm{m}^{-1}\right) \cdot{ }^{[126,151]}$ In general, the surface energy decreases with increasing temperature. Several studies have shown the temperature dependence of the surface energy of PS and PNIPAM.[152-154] Here, the temperature dependency is pronounced for the PS domains resulting in lower areas as before. With increasing temperature, the formation of the darker areas is reinforced. Initially, it was dot-like areas which converted to worm-like structure in a light matrix. By increasing the temperature up to $200{ }^{\circ} \mathrm{C}$ the self-assembled structure completely disappeared. Large dark holes formed which indicate a macrophase separation. It can be assumed, that the high annealing temperature led to degradation of the thin polymer films. 


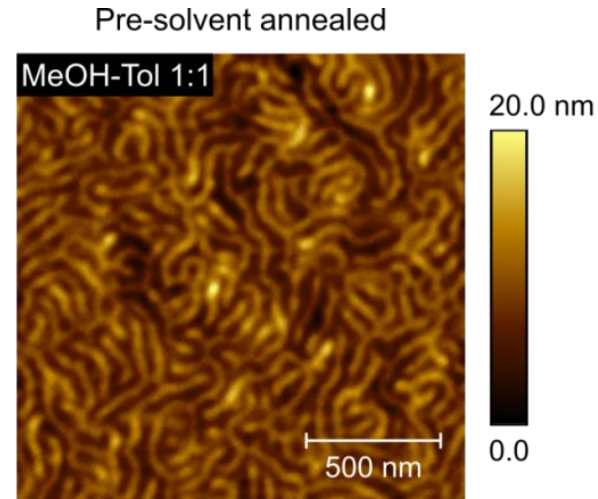

Combined with thermal annealing at different temperatures
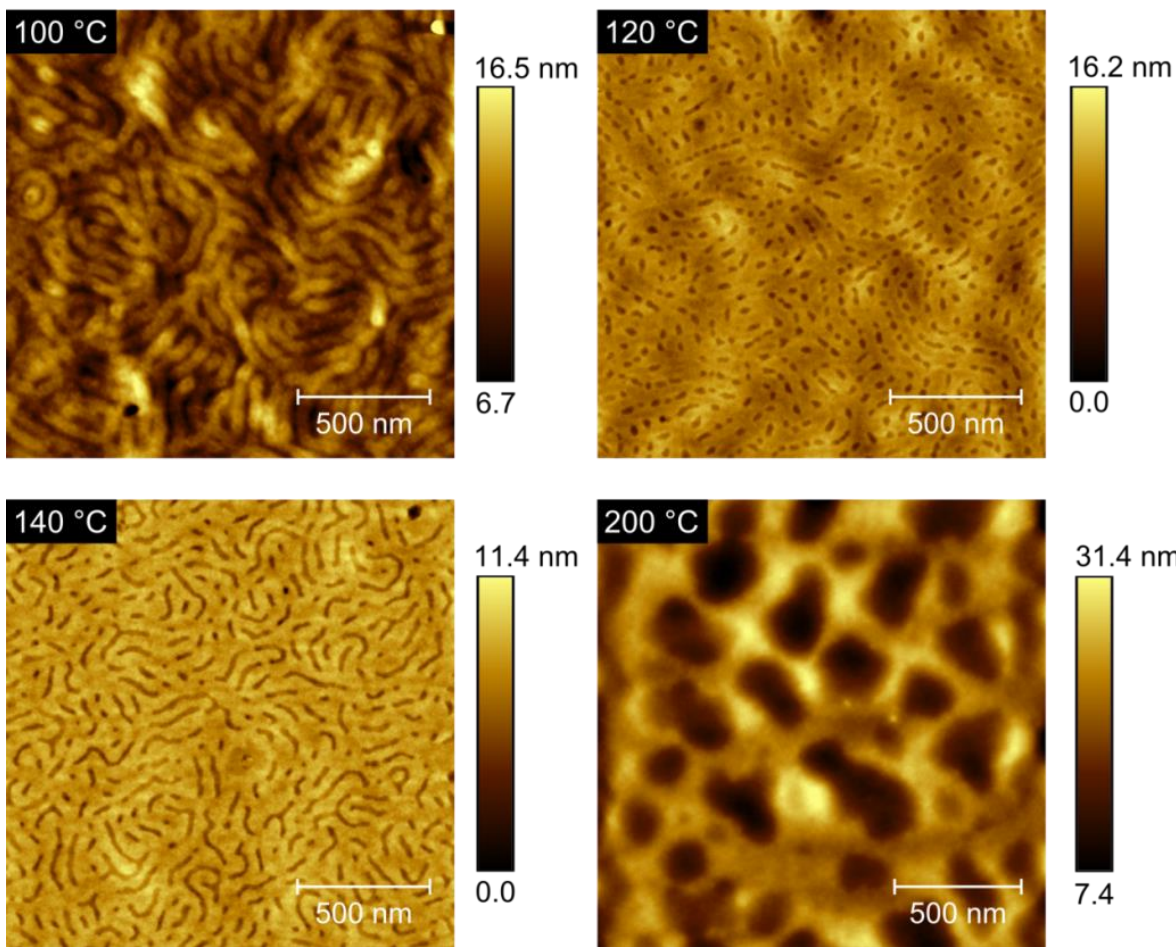

$31.4 \mathrm{~nm}$

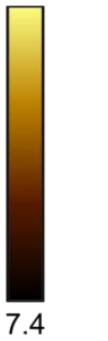

Figure 2.41. Pre-solvent annealed film of sample $\mathbf{X}$ using MeOH-Tol 1:1 (v:v) and following thermal annealing at different temperatures.

The previous experiments showed that the structure of the solvent annealed films can be converted by thermal annealing. Of further interest was wheter the morphology before thermal annealing (see Figure 2.42a) could be regained by solvent annealing. As shown in Figure 2.42c the self-assembled structure could be recovered. The regained structure, parallel cylinders or 
perpendicular lamellae, were less organized and thicker than before thermal treatment (see Figure 2.42a). After thermal annealing, the chain stretching was promoted resulting in thicker domains. The domain size is increased to a value of $(74 \pm 3) \mathrm{nm}$. It has to be mentioned that the samples were probed at different areas after each annealing step. To ensure that the thin film showed the same morphology over the entire surface, at least three different areas were probed. In addition, it was investigated if the converted structure can be extended. The sample was first solvent annealed with methanol-toluene $1: 1(\mathrm{v}: \mathrm{v})$ and then thermally annealed at $140{ }^{\circ} \mathrm{C}$ up to $180^{\circ} \mathrm{C}$ in $20^{\circ} \mathrm{C}$ steps. After every thermal annealing step the surface morphology was determined via AFM. The corresponding images are displayed in Figure 2.42. After repeating thermal annealing the same selfassembled structure (Figure 2.42d) is visible as after the first thermal treatment (Figure 2.42b). Further successive heating of the thin film leads to distinct growing of the worm-like dark areas, as shown in Figure 2.42d and e. The worm-like areas change to a fingerprint-like structure. The domain size is decreased to a value of $(45 \pm 1) \mathrm{nm}$ (see Figure 2.43). The temperatures above the glass transition (Table 2.8) result in higher chain mobility and thus the dark area structure is extended. The combination of solvent annealing with successive thermal annealing leads to higher ordered domains. The enhanced ordering due to this thermo-solvent process could be attributed to the relaxation of the film by the pre-solvent annealing and the higher chain mobility at temperatures above $T_{\mathrm{g}}{ }^{[}{ }^{106]}$ 

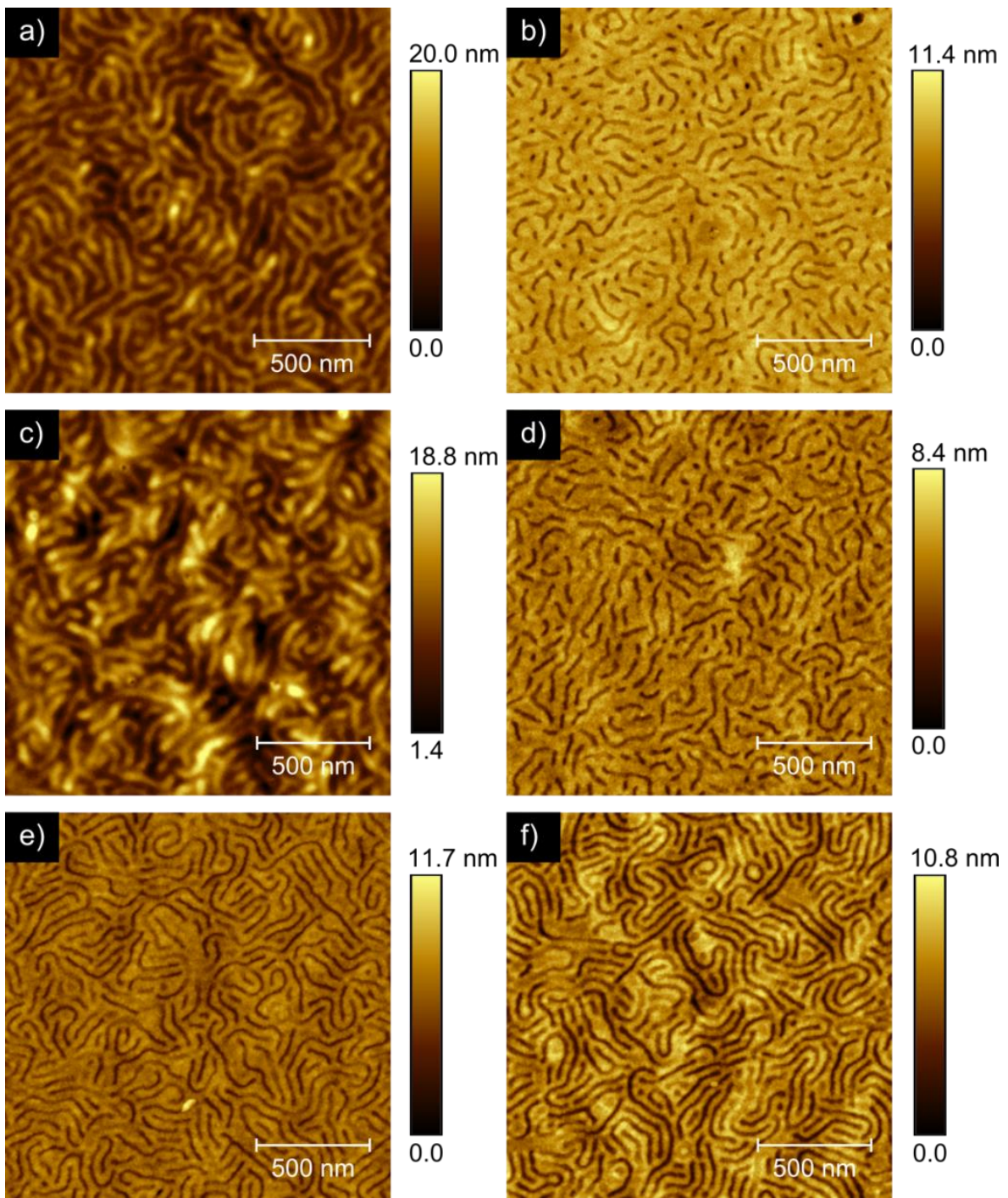

Figure 2.42. Thermosolvent annealing of sample $\mathbf{X}$. a) solvent annealing using MeOH-Tol 1:1 (v:v), b) solvent annealing combined with thermal treatment at $140^{\circ} \mathrm{C}, \mathrm{c}$ ) repeating solvent annealing using MeOH-Tol 1:1 (v:v) after treatment (b), d) thermal annealing at $140{ }^{\circ} \mathrm{C}$ after (c), e) successive thermal annealing of sample (d) at $160^{\circ} \mathrm{C}$, f) further thermal annealing of probe (e) at $180^{\circ} \mathrm{C}$. 


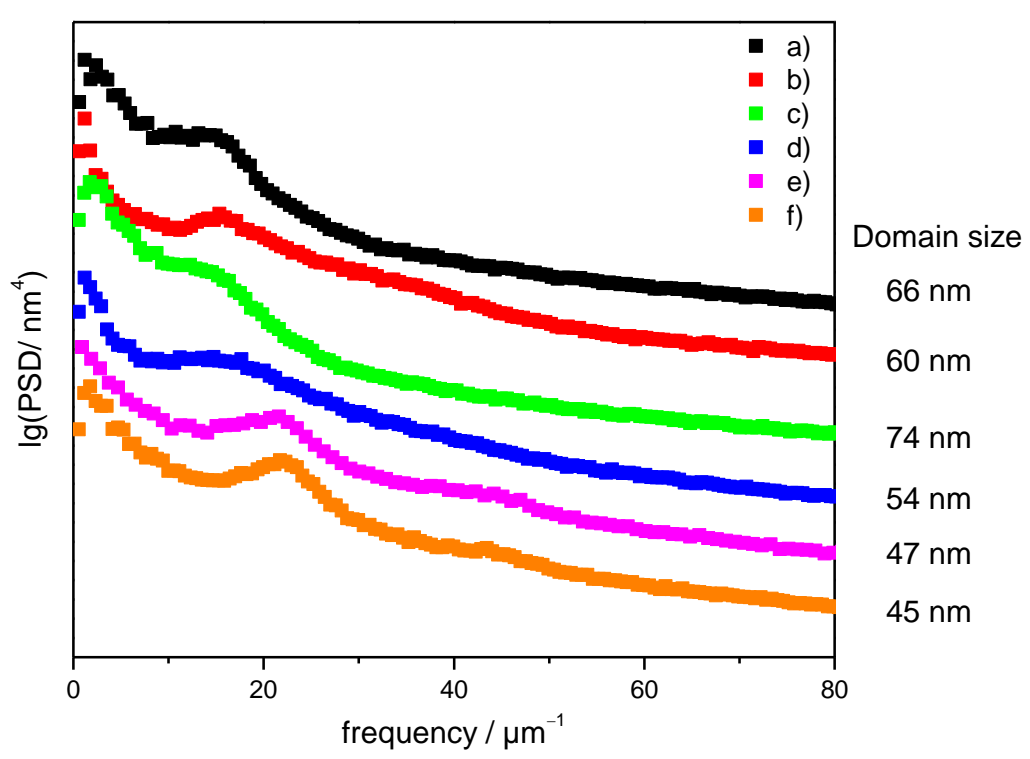

Figure 2.43. Two-dimensional PSD distribution of the AFM height profiles shown in Figure 2.42. The assignment of the domain curves is adapted from Figure 2.42. The curves have been vertically shifted for clarity. Domain size determined via baseline correction and following Lorentzian fit of the PSD distribution.

After the thermo-solvent process the thin film (Figure 2.42f) was solvent annealed using methanol-toluene $1: 1(\mathrm{v}: \mathrm{v})$ to ensure the switchable behavior of the morphologies. In Figure 2.44 a set of images after repeating solvent annealing is displayed using the PFQNM measuring mode. In the topography image (Figure 2.44b) a different self-assembled structure was obtained as before (Figure 2.42a). Unordered micelle-like light dots within a dark matrix are visible. A possible explanation is that the polymer was partially degraded which would result in a different polymer composition and thus another morphology. To exclude degradation size-exclusionchromatography experiments of the annealed bulk material of sample $\mathbf{X}$ were performed. The bulk material was thermally annealed in the same manner as the thin films. Figure 2.45 shows the size-exclusionchromatograms. The bulk material is stable up to $200{ }^{\circ} \mathrm{C}$, only a slight shift to lower molar mass range is visible. As no significant decrease in molar mass was observed, partial degradation was ruled out. It cannot be excluded that different effects are present in the thin film than in the examined bulk material. As described earlier, the surface energy decreases 
with increasing temperature. The effect is pronounced in the PS domains which could cause alignment change from parallel to perpendicular cylinders. This effect suggests that the structure before is lying cylinder and not perpendicular lamellae.

Since the film shows inhomogeneity of the topography image, the adhesion image (Figure 2.44c) and logarithmic Young's modulus map (Figure 2.44d) are also presented. It has to be mentioned that the scale bar for those image is just a relative scale because no appropriate calibration for the measurement was available. The logarithmic DMT modulus map shows stiff (light) dots surround by a softer (dark) matrix. In the adhesion image, the dots appear darker with a lower adhesion than the matrix. Due to the fact that PS has a higher Young's modulus than PNIPAM, the light dots can be assigned to PS (stiffer areas) and the dark matrix PNIPAM (softer areas).

after thermo-solvent process
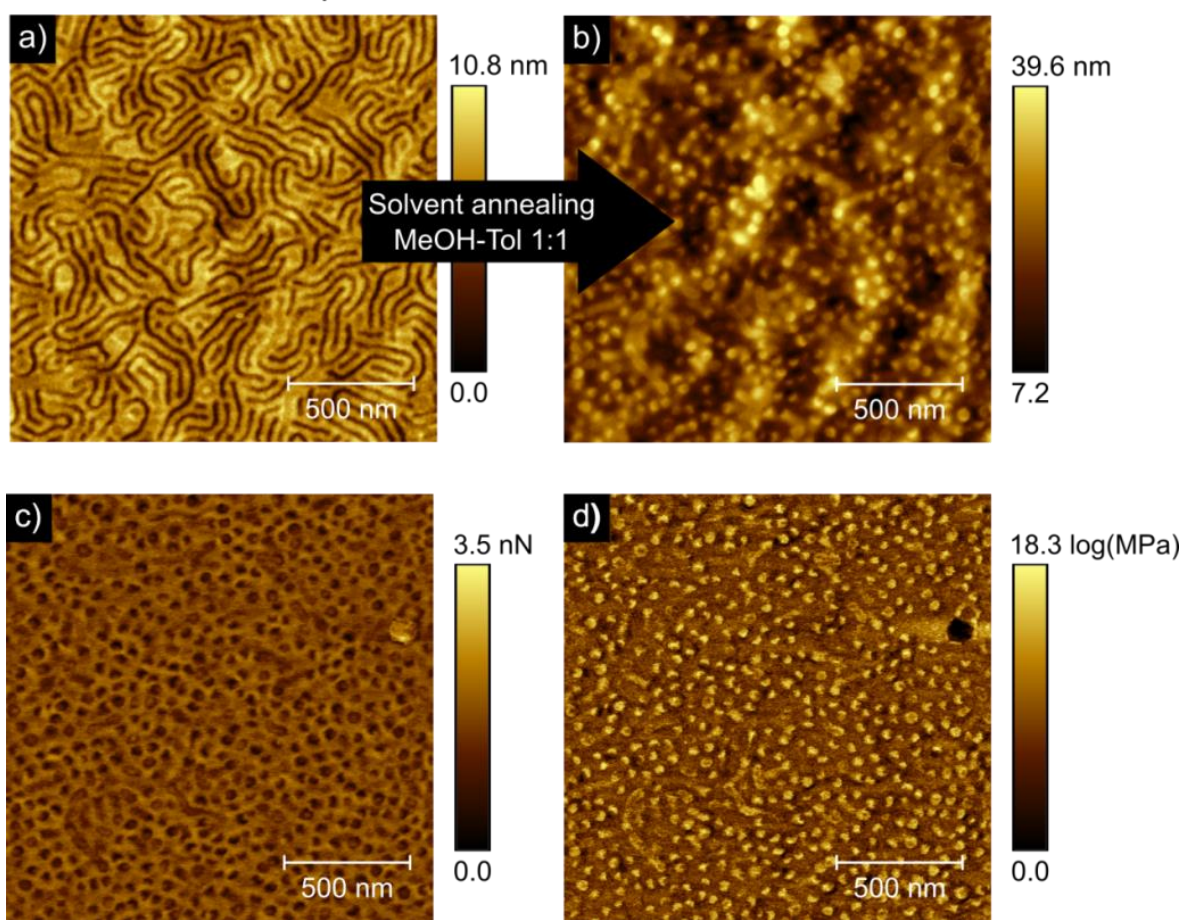

Figure 2.44. AFM topography images of sample $\mathbf{X}$ a) after thermos-solvent treatment described in Figure 2.42 (top) and b) following solvent annealing using MeOH-Tol 1:1 (v:v) (bottom). The image b) displays the height profile of the annealed film, c) the adhesion and d) $\log (\mathrm{DMT})$ image with a relative scale. 


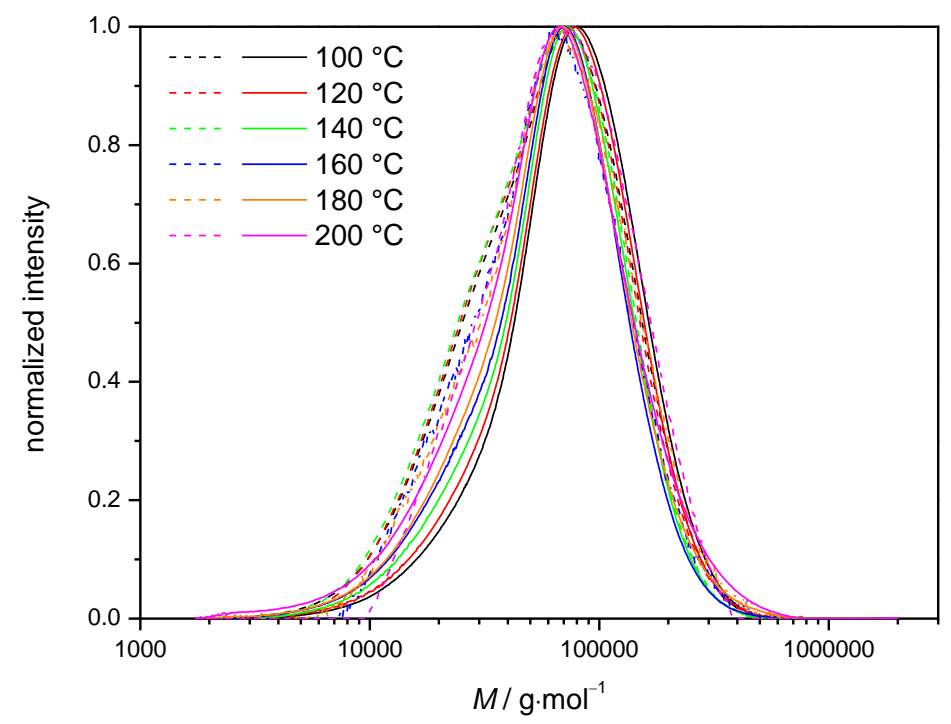

Figure 2.45. Size-exclusion-chromatograms of sample $\mathbf{X}$ after thermal annealing at different temperatures. UV (dashed line, wavelength of $\lambda=310 \mathrm{~nm}$ ) and RI (solid line) signals are displayed.

\section{Cleavage Products}

Here, the microphase separation behavior of the cleavage product which corresponds to the $\mathrm{AB}$ block copolymer is investigated. The multiblock copolymer $\mathbf{X}$ was cleaved by an excess of radicals and thin films were prepared and solvent annealed using methanol-THF and methanol-toluene 1:1 (v:v) mixtures as described above. Figure 2.46 shows the resulting AFM topography images. For clarity, the phase separation structure of the annealed multiblock copolymer is displayed as well. The asspun film (see Figure 2.46b) shows nicely ordered hexagonally packed cylinders are orientated perpendicular to the substrate. While the corresponding film of the ABA block copolymer exhibit micelle-like objects which can be assigned to unordered cylinders. This difference can be attributed to the higher chain mobility of polymers with lower segments number. The thin films were also solvent annealed using different solvent mixtures. After solvent annealing using methanol-THF 1:1 (v:v), the dissected polymer orientates into a mixed morphology of perpendicular and parallel cylinders. A morphology is formed after solvent annealing using methanol-toluene 1:1 (v:v). The previous perpendicular cylinders seemed to be partially melted together by the solvent vapor. The change of 
the solvent mixture from a rather PNIPAM-selective to PNIPAM- and PS-selective mixture provides a competition between the solvent vapor on the thin film. Both blocks are swollen selectively while with methanol-THF only the PNIPAM block is swollen selectively. Due to this the morphology of the thin film results in a non-equilibrium state and thus a perforated film with cylinders is obtained. It can be assumed that higher annealing times and increasing of the PS-selective solvent, toluene, would lead to enhanced arrangement. 

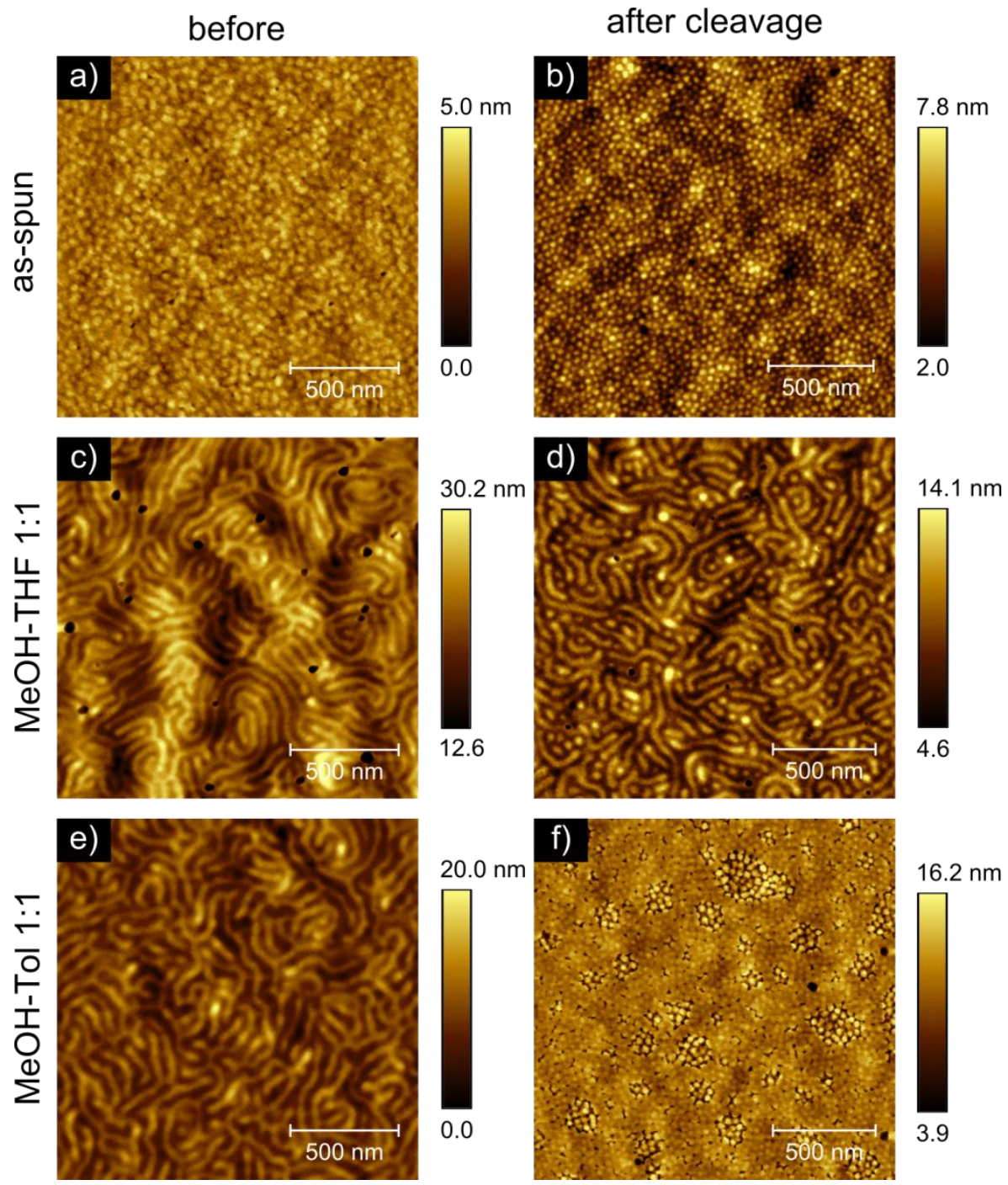

$16.2 \mathrm{~nm}$

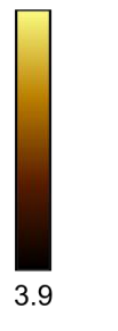

Figure 2.46. AFM topography images of the cleavage product of multiblock copolymer $\mathbf{X}$ b) as-spun and after solvent annealing using d) MeOH-THF 1:1 (v:v) and $\mathrm{f}) \mathrm{MeOH}-\mathrm{Tol}$ 1:1 (v:v). For comparison the corresponding topographies of multiblock copolymer $\mathbf{X}$ are displayed as well a) as-spun and after solvent annealing using c) MeOH-THF 1:1 (v:v) and e) MeOH-Tol 1:1 (v:v). 


\subsection{Conclusions}

Multiblock copolymers $m$-(PS- $b$-PBA) and $m$-(PS- $b$-PNIPAM) were successfully synthesized using a polyfunctional RAFT agent. Morphology behavior of their thin films after different annealing techniques was investigated with AFM. Thermal annealing was found to be a suitable annealing process for the $m$-(PS- $b$-PBA) system and solvent annealing for $m$-(PS- $b$-PNIPAM). For the $m$-(PS- $b$-PBA) system, different morphologies were obtained depending on the volume fraction of PBA. Perpendicular cylinders were observed with a PBA volume fraction of $f_{\mathrm{PBA}}=0.69$, whereas at a higher volume fraction the morphology orientated to parallel cylinders.

Solvent annealing was used to control the morphology of the $m$-(PS- $b$-PNIPAM) system. The influence of solvent type, solvent ratio, annealing time and film thickness was examined. Annealing with selective solvent mixture of methanol-THF and methanol-toluene were found to form perpendicular hexagonally packed PS cylinders in a PNIPAM matrix for a PNIPAM volume fraction $0.59<f_{\text {PNIPAM }}<0.77$ independent of the average block number. Using only one type of solvent resulted in different morphologies. THF led to an unordered state. Methanol showed weakly oriented perpendicular cylinders. An order-order transition occurred to perforated lamellae using toluene which increased the effective block volume of PS. This displays the importance of solvent choice for direct control to a specific morphology. In addition, the film thickness was found to influence the morphology. By increasing the thickness, movement of the film is reduced and formation of the microdomains is decelerated.

ABA block copolymer of PS-PNIPAM showed similar dependence of selective of solvent (mixture) as the multiblock copolymers. Pure solvents like THF and toluene did not change the obtained as-spun micellar-like morphology. Using methanol as a solvent parallel cylinders were formed. By assimilating the selective solvent mixture to the polymer composition well-ordered parallel cylinders were observed. In addition, a combination of pre-solvent and gradual thermal annealing was applied on the thin films which resulted in an inversion of the initial morphology and higher order.

These results demonstrate that despite the complexity of multiblock copolymers, highly ordered films were obtained within one hour by appropriate solvent annealing or thermal annealing conditions. This result opens up the pathway for applications as structure directing agents in the formation of mesoporous thin films. 


\section{Novel Mesoporous Silica Thin Films via Multiblock Copolymers}

\subsection{Preface}

Porous materials show unique properties due to their well-defined geometry and size. At first, the preparation of structured materials was restricted to pores on the sub-nanometer scale. The introduction of supramolecular pore templating by the Mobil group and Yanagisawa et al., overcame these size limitations and led to a new family of porous silica compounds with a pore size ranging from 2 to $50 \mathrm{~nm} \cdot{ }^{66,7,155,156]}$ This procedure enabled the development of materials for practical applications such as catalysis, adsorption, separation, sensing, medical usage, ecology and nanotechnology. ${ }^{[155,157,158]}$ The synthesis strategy for these mesoporous materials is based on the combination of sol-gel chemistry and selfassembly of organic templates (surfactants) and is not only restricted to silica oxides. Several studies demonstrated well-defined structures using titanium or zirconium oxides.[155,159,160] There are four different synthetic pathways to obtain mesostructured materials: 1) direct precipitation, 2) true liquid crystal templating, 3) evaporation-induced self-assembly (EISA) and 4) exotemplating. The EISA method is arguably the most established and efficient procedure compared to other procedures available. In this process a solvent evaporates from a diluted solution of an inorganic precursor, an organic template and other additives. This results in the formation of a hybrid mesostructured phase and is followed by template removal. Due to the versatility of the process the formation of the mesostructured phase can be tuned by template structure, organic/inorganic molar ratio and coating procedure. By this method mesoporous silica thin films exhibiting unimodal pore size distribution, high surface area and modifiable pore size were synthesized. For example, silica materials with a pore size of 1.5-4 nm were prepared using ionic surfactants such as alkytrimethylammonium bromide or chloride. Amphiphilic block copolymers are an especially unique class of structuring agent due to their inherent ability to phase separate into nanodomains. Mostly AB- and ABA-block copolymers like polyethylene oxide- $b$-polypropylene- $b$-polyethylene oxide are used to form 
mesoporous materials with a high degree of organization, porosity and large pore size $(2-30 \mathrm{~nm})$. $^{[155]}$ In general, block copolymers offer an attractive organic template which is tunable in molar mass, block number and composition. In this context, symmetrical multiblock copolymers have not been exploited so far. The usage of multiblock copolymers could extend the range of accessible pore sizes, structures and stability.

In this chapter, a strategy is introduced to synthesize mesoporous silica thin films using amphiphilic multiblock copolymers prepared via RAFT polymerization as a template. For the first time, it is demonstrated that these multiblock copolymers are a universal tool of structure directing agents for mesoporous materials. The pore size distribution and porosity of the thin films were characterized by scanning electron microscopy (SEM) and AFM. Interconnectivity was investigated by nitrogen adsorptiondesorption isotherms and film thickness was determined using ellipsometry.

\subsection{Classification of porous materials}

Porous materials can be divided into three categories by their pore size. Micropores are present if their size is less than $2 \mathrm{~nm}$. Mesostructured materials exhibit pore sizes from 2 to $50 \mathrm{~nm}$ and macroporous materials display pore size larger than $50 \mathrm{~nm}$. In order to distinguish between different pores IUPAC introduced the classification depending on the availability to an external fluid.[161] Figure 3.1 (left) illustrates the crosssection of a porous material. Closed pores (a) do not show any access for external fluids and thus are completely isolated from neighboring pores. The counterparts to these are the group of open pores $(b, c, d, e$, and $f$ ). These can also be subdivided into blind pores ( $b$ and $f$ ) containing only one open end and through pores (e) with at least two open ends. Furthermore, the pores can be classified by their shape. They can be distinguished into cylindrical (c and f), ink-bottle shaped (b), funnel shaped/conical (d), slitsshaped or interstices pores. ${ }^{[161]}$
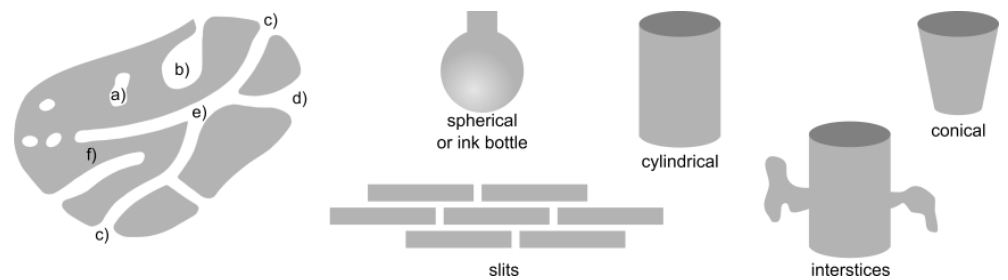

Figure 3.1. Schematic cross-section of porous solid and possible shapes of pores.[161,162] 
Porous materials can be characterized by physical adsorption of gas onto the surface of the pores. Depending on the form of the isotherms, conclusions on the shape of the pores can be drawn. Furthermore, pore size and surface area can be determined. According to IUPAC the physisorption isotherms can be divided into eight different groups (see Figure 3.2).[163]

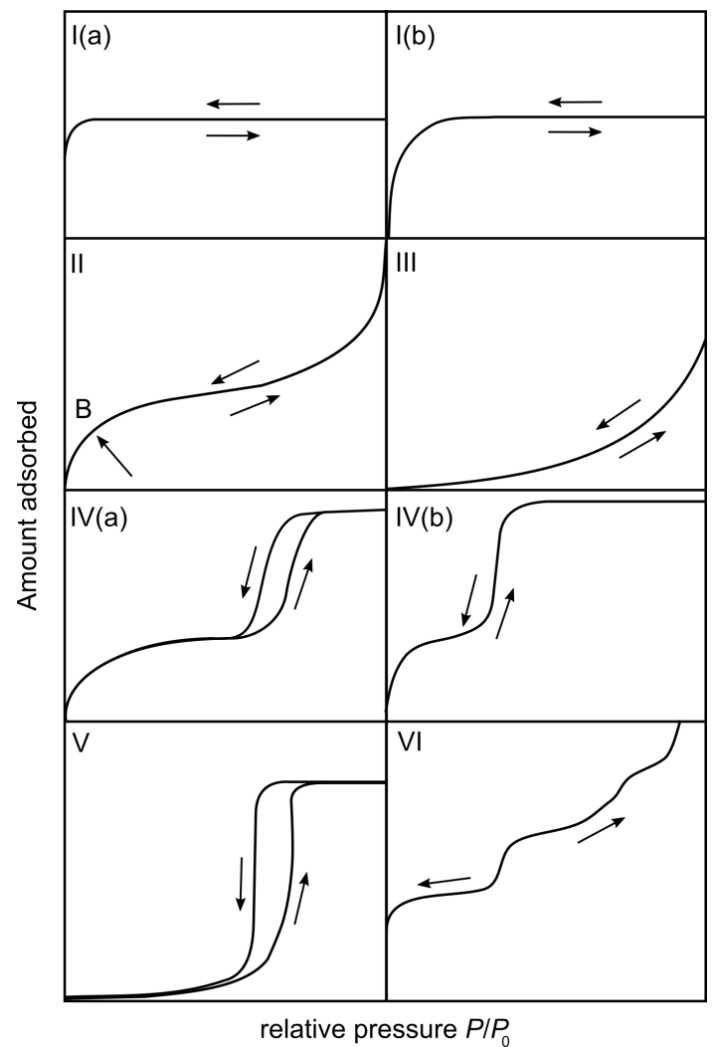

Figure 3.2. Classification of physisorption isotherms.[163]

In general, hysteresis loops are caused by the adsorption metastability and/or by network effects. Figure 3.3 (right) shows the hysteresis behavior of ink-bottle pores. At low relative pressure the pores are empty. By increasing the relative pressure monolayer-multilayer adsorption takes place at the walls of the pores which is followed by pore condensation. The plateau is reached when the pores are completely filled. During desorption the pores remain filled until the narrow neck is empty at lower relative pressure which results in the observed hysteresis loop. The hysteresis loop can also be divided into six different groups (see Figure 3.3, left). 

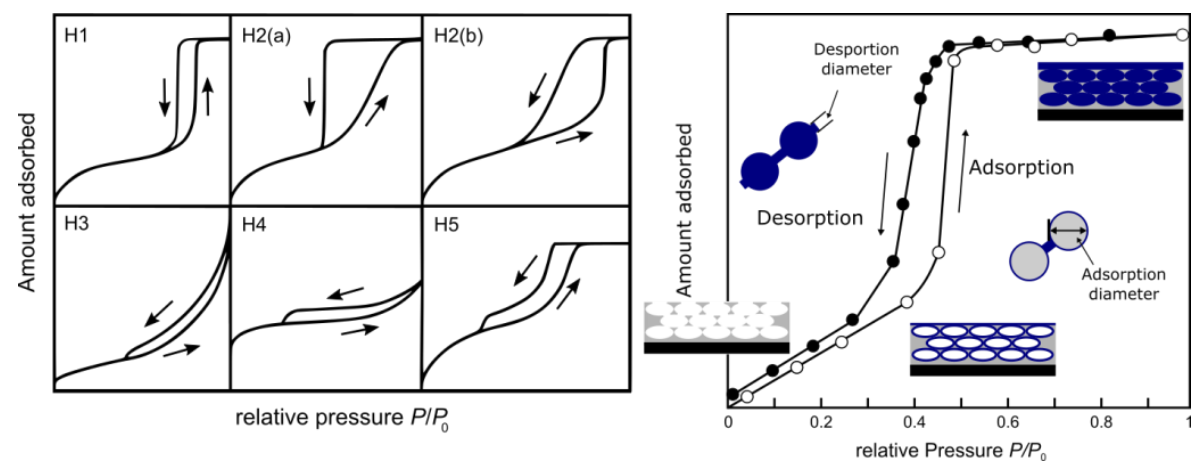

Figure 3.3. Classification of hysteresis loops (left) and illustration of the adsorption-desorption behavior of ink-bottle shaped porous materials (right).[163,164]

\subsection{Fundamentals of Mesostructured Silica Thin Films}

The combination of sol-gel chemistry and the self-assembly process of organic templates (surfactants) gives unique control over the mesoporous and mesostructured phases at the nanoscale $(2-50 \mathrm{~nm})$. The mesostructured hybrid phase is formed by co-assembling of inorganic precursor and organic supramolecular template. In a second step the template is removed which leads to the actual mesoporous material.

The sol-gel process is based on the controlled polymerization of inorganic molecular precursors in mild conditions. In general, these precursors have a chemical structure of either $\mathrm{MX}_{\mathrm{n}}($ e.g. $\mathrm{M}=\mathrm{Si}, \mathrm{Ti}, \mathrm{Zr}, \mathrm{Al}$ ) with $\mathrm{X}$ a hydrolysable group like chloride or MOR with $\mathrm{OR}$ an alkoxide group. The synthesis is normally performed in hydroalcoholic or aqueous medium at room temperature. Scheme 3.1 shows the reactions which take place in the sol-gel process.
(a) $\mathrm{M}-\mathrm{OR}+\mathrm{H}_{2} \mathrm{O} \longrightarrow \mathrm{M}-\mathrm{OH}+\mathrm{ROH}$
(b) $\mathrm{M}-\mathrm{OH}+\mathrm{XO}-\mathrm{M} \longrightarrow \mathrm{M}-\mathrm{O}-\mathrm{M}+\mathrm{X}-\mathrm{OH}, \quad \mathrm{X}=\mathrm{H}$ or $\mathrm{R}$
(c) $\mathrm{M}-\mathrm{OH}+\mathrm{M}-\mathrm{OX} \longrightarrow \mathrm{M}-(\mathrm{OH})-\mathrm{M}-\mathrm{OX}, \quad \mathrm{X}=\mathrm{H}$ or $\mathrm{R}$

Scheme 3.1. Generalized scheme of the sol-gel process with the reactions (a) hydrolysis, (b) condensation and (c) olation.[110]

First, the alkoxide group that is attached to the metal species is replaced by a hydroxyl group during hydrolysis. This species can react again in two different ways with another metal center (see Scheme 3.1b and c). An oxo 
bridge is formed by condensation of the hydroxylated metal species with another metal center. During the reaction, either water (oxolation) or alcohol (alcoxolation) is eliminated. Hydroxo bridges are formed in the olation reaction. ${ }^{[110,165]}$ The size, shape and philicity of the inorganic building blocks can be adjusted by chemical control over the sol-gel reactions. The philicity of the inorganic species plays an important role in modifying the interaction with the organic structure directing agent. In this work, silica is selected as the inorganic metal species. It is known that silicabased systems require low $\mathrm{pH}$ values to catalyze the hydrolysis reaction. Additionally, the condensation is minimized at $\mathrm{pH}$ values of $\mathrm{pH}=1-3$.[110]

Commonly, the structure directing agent is either an ionic surfactant like cetyltrimethylammonium bromide (CTAB) or a non-ionic amphiphilic molecule such as a block copolymer. The mesostructured oxides prepared by ionic surfactants show some limitation compared to those prepared by using block copolymer. On the one hand, the wall thickness is limited to $0.8-1.3 \mathrm{~nm}$. On the other hand, pore sizes greater than $5 \mathrm{~nm}$ are only accessible by employing swelling agents such as 1,3,5-trimethylbenzene. ${ }^{[158]}$ In this work, amphiphilic block copolymers will be used as organic building units. Since block copolymers are capable of phase separating into organized structures, they are an import class of surfactants. The properties of the block copolymers can be easily tuned by solvent composition, molar mass or the macromolecular architecture. Furthermore, larger pore sizes $(5-100 \mathrm{~nm})$ are available and a diversity of pore connectivities has been explored.[166] In literature, mostly AB- and ABA-block copolymers are employed as structure directing agents. Usually, polyethylene oxide (PEO) or polyacrylic acid (PAA) are used as hydrophilic blocks and polystyrene (PS) or polypropylene (PPO) as hydrophobic blocks.

In aqueous solutions above the critical micelle concentration $(\mathrm{cmc})$ surfactants assemble into micelles and form either spherical or cylindrical structures. The hydrophilic part is arranged towards the water contact interface and the hydrophobic part is located in the micellar interior. Increasing the concentration of the surfactant leads to periodic hexagonal, cubic or lamellar mesophases.[167] The combination of a surfactant or block copolymer and sol-gel precursor solution provides a co-assembled hybrid mesophase. After subsequent removal of the surfactant periodic, mesoporous materials are formed.

In this work the EISA process (see Scheme 3.2) is employed and will be highlighted in the following section. Brinker's and Ozin's group introduced this method which is based on solvent evaporation from dilute solutions of inorganic precursors, template agent and other additives to form hybrid mesostructured phases.[167-171] 


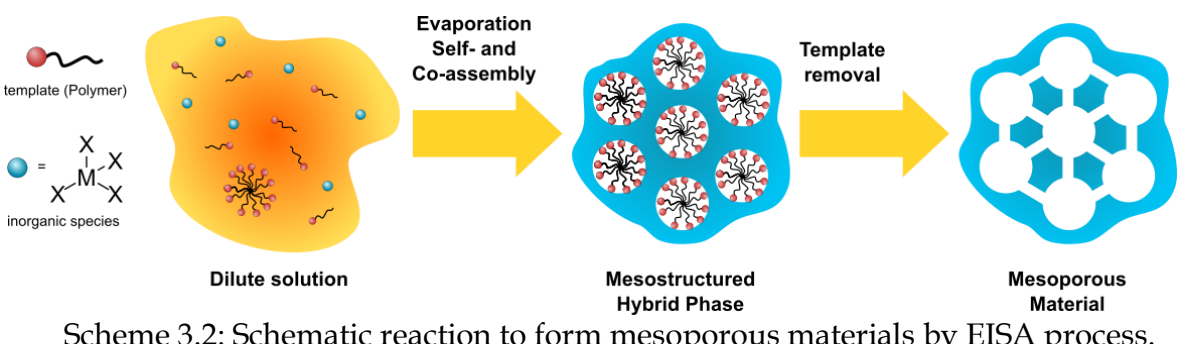

In this technique a mixture of sol-gel precursors such as water, alcohol (usually ethanol), acid (typically hydrochloric acid) and a metal alkoxide or metal salt is combined with a surfactant. The initial concentration of the surfactant in the solution is much lower than the critical micelle concentration $(\mathrm{cmc})$. The solution is deposited on a substrate in order to form mesoporous thin films (MTFs) by spin or dip coating. The schematic process of EISA using dip coating is displayed in Figure 3.4. During depositing of the solution on a substrate, the volatile components, alcohol, hydrochloric acid and water, evaporate at the air-film interface. The concentration of the metal oxide oligomers and the non-volatile surfactant increases during the initial stage (10-30 s). Due to the increase of the surfactant concentration, a liquid crystal (LC) phase of the organicinorganic hybrid is formed. When the concentration of the surfactant equals the cmc, micelles are obtained and a Modulable or Tunable Steady State (MSS/TSS) is reached. This state can be described as an organized LC mesophase in which the inorganic network is not fully condensed. During this stage an equilibrium of the solvent and water molecules in the film with the environment exists. The duration usually depends on the atmospheric relative humidity $(\mathrm{RH})$. In the final step, the template is removed in order to reach porosity and fully condense the inorganic network. Commonly, the template is removed by calcination in the temperature range from 400 to $550{ }^{\circ} \mathrm{C}$. However, milder treatments such as solvent extraction or degradation of organic materials by $\mathrm{UV}-\mathrm{O}_{3}$ are also available. Three import factors influence the final mesostructure: 1) ratio of the surfactant to the metal oxide precursor, 2) nature of the precursor and 3) the RH value.[171,172] 


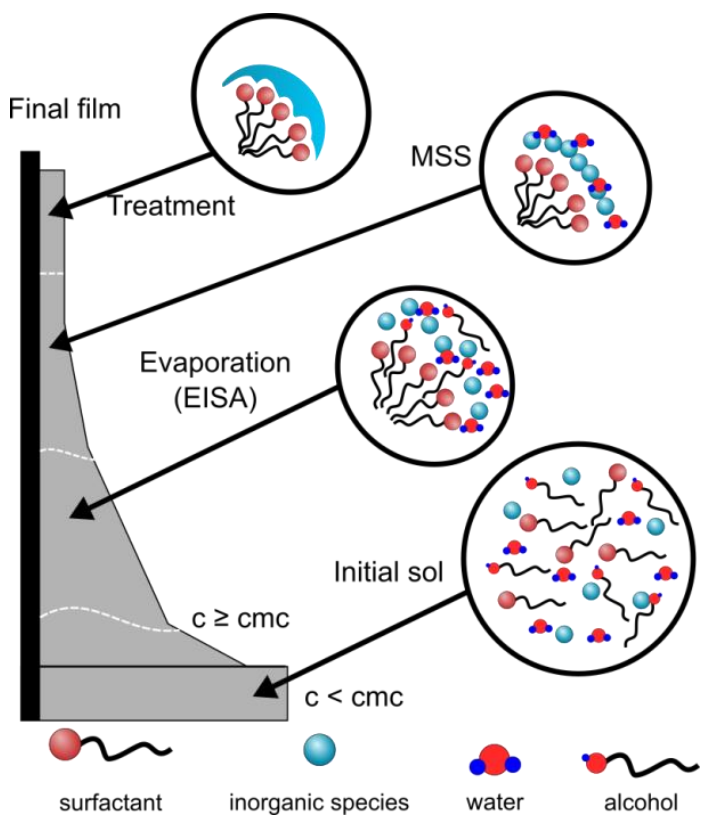

Figure 3.4. Reaction steps in the formation of mesostructured thin films by dip coating.

\subsection{Synthesis of Mesoporous Silica Thin Films by Multiblock Copolymers}

In this chapter the synthesis strategy to mesoporous silica thin films using multiblock copolymers will be described. The amphiphilic multiblock copolymers (MBC) consist of poly( $N$-isopropylacrylamide) as the hydrophilic part and polystyrene as the hydrophobic part. They are a suitable system to study their role as structure directing agent for the preparation of mesoporous silica thin films. The microphase separation behavior of the amphiphilic multiblock copolymer $m$-(PS- $b$-PNIPAM) into well-defined morphologies has been discussed in detail in section 2.5.2. For a better overview, the chemical properties of the utilized multiblock copolymers are collated in Table 3.1. 
Table 3.1. Amphiphilic multiblock copolymer $m$-(PS- $b$-PNIPAM) with different PNIPAM comonomer composition $\phi_{\text {PNIPAM }}$ (determined via ${ }^{1} \mathrm{H}-\mathrm{NMR}$ spectroscopy) or volume fraction $f_{\text {PNIPAM }}$ employed as structure directing agent for the preparation of mesoporous silica thin films. $\bar{M}_{\mathrm{n}, 0}$ denotes the number average molar mass prior to cleavage of the MBC and $\bar{M}_{\mathrm{n}, 1}$ after cleavage. For determination of the average number of blocks $b$ and segments $s$ equation (2.3) and (2.4) were used.

\begin{tabular}{cccccccccc}
\hline sample & $\begin{array}{c}\overline{\boldsymbol{M}}_{\mathbf{n}, \mathbf{0}} / \\
\mathbf{g} \cdot \mathbf{m o l}^{-1}\end{array}$ & $\boldsymbol{\Xi}$ & $\begin{array}{c}\overline{\boldsymbol{M}}_{\mathbf{n}, \mathbf{1}} / \\
\mathbf{g} \cdot \mathbf{m o l}^{-1}\end{array}$ & $\boldsymbol{\Xi}$ & $\boldsymbol{\phi}_{\text {PNIPAM }}$ & $f_{\text {PNIPAM }}$ & $\boldsymbol{N}_{\text {TTC }}$ & $\boldsymbol{b}$ & $\boldsymbol{s}$ \\
\hline V & 127900 & 2.32 & 25200 & 1.90 & 0.78 & 0.77 & 4 & 5 & 9 \\
IX & 54600 & 1.46 & 13650 & 1.53 & 0.75 & 0.74 & 3 & 4 & 7 \\
X & 52400 & 1.77 & 34300 & 1.70 & 0.55 & 0.54 & 1 & 2 & 3 \\
XI & 64500 & 1.45 & 32000 & 1.40 & 0.71 & 0.71 & 1 & 2 & 3 \\
\hline
\end{tabular}

As the inorganic precursor, the well-studied tetraethyloxysilane (TEOS) is employed. Usually, ethanol is used as a solvent because of its good wettability properties with glass or silicon substrates.[173] However, THF was selected as the solvent due to low solubility of the MBC in ethanol at higher molar masses and an upper critical solution temperature (UCST) of PNIPAM in ethanol/water solutions. ${ }^{[174]}$ THF can be considered a good solvent for both blocks. Furthermore, THF is water-soluble and is more volatile than water. In literature, several reports show the successful preparation of mesoporous silica thin films using THF.[175,176]

Scheme 3.3 shows the developed general procedure. An acidcatalyzed TEOS-solution was prepared and mixed with a solution of the MBC. This solution was aged for a period of time. Then, the thin films were prepared by depositing the solution onto silicon wafer substrate using spin coating in a controlled environment $(\mathrm{RH}<5 \%$, RT). In order to find the appropriate conditions for a good mesoporous silica films, the procedure was varied in structural promoting workup, template removal and $\mathrm{MBC}$ ratio. The employed conditions are summarized in Table 3.2.

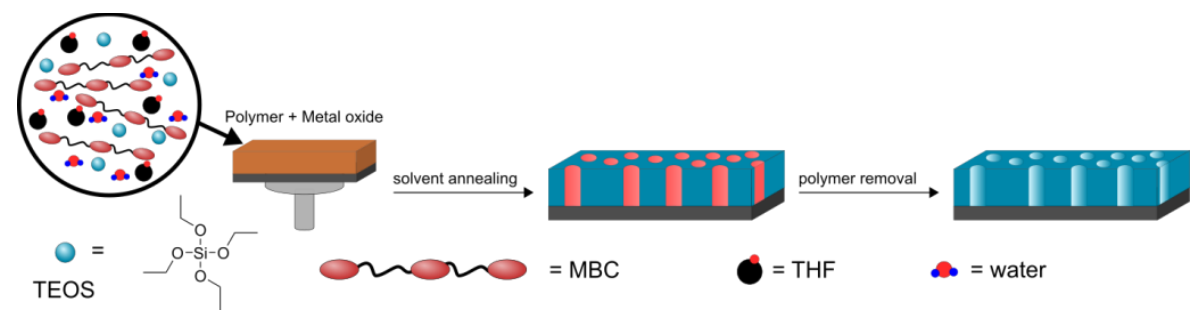

Scheme 3.3. Applied EISA method by spin coating a solution of acid-catalyzedTEOS, THF, water and MBC onto a silicon wafer substrate. Pattern formation was achieved by solvent annealing with $\mathrm{MeOH}-\mathrm{THF}$ mixture and final polymer removal. 
Table 3.2. Amount of multiblock copolymer (MBC), stirring time for the solution, structure promotion workup using solvent annealing (SVA) and template removal procedure for all samples.

\begin{tabular}{ccccccc}
\hline sample & $\begin{array}{c}\text { MBC } \\
\text { template }\end{array}$ & $\begin{array}{c}\text { MBC } \\
\text { /g }\end{array}$ & $\begin{array}{c}\text { final } \\
\text { solution } \\
\text { stirring }\end{array}$ & $\begin{array}{c}\text { structural } \\
\text { promoting } \\
\text { workup }\end{array}$ & $\begin{array}{c}\text { template } \\
\text { removal }\end{array}$ & $\begin{array}{c}\text { porous } \\
\text { structure }\end{array}$ \\
\hline MP1 & X & 0.02 & $1 \mathrm{~d}$ & SVA & Plasma & Yes \\
MP2 & X & 0.02 & $1 \mathrm{~d}$ & SVA & $450{ }^{\circ} \mathrm{C}$ & Yes \\
MP3 & X & 0.02 & $1 \mathrm{~d}$ & None & Plasma & No \\
MP4 & X & 0.02 & $1 \mathrm{~d}$ & None & $450{ }^{\circ} \mathrm{C}$ & Yes \\
MP5 & IX & 0.02 & $1 \mathrm{~d}$ & SVA & Plasma & Yes \\
MP6 & IX & 0.02 & $1 \mathrm{~d}$ & SVA & $450{ }^{\circ} \mathrm{C}$ & Yes \\
MP7 & IX & 0.02 & $1 \mathrm{~d}$ & None & $450{ }^{\circ} \mathrm{C}$ & No \\
MP8 & V & 0.02 & $1 \mathrm{~d}$ & SVA & Plasma & Yes \\
MP9 & V & 0.02 & $1 \mathrm{~d}$ & SVA & $450{ }^{\circ} \mathrm{C}$ & No \\
MP10 & V & 0.02 & $1 \mathrm{~d}$ & None & Plasma & Incomplete \\
MP11 & V & 0.02 & $1 \mathrm{~d}$ & None & $450{ }^{\circ} \mathrm{C}$ & Yes \\
MP12 & IX & 0.02 & $3 \mathrm{~d}$ & SVA & Plasma & Yes \\
MP13 & V & 0.02 & $3 \mathrm{~d}$ & SVA & Plasma & Yes \\
MP14 & IX & 0.02 & $7 \mathrm{~d}$ & SVA & Plasma & No \\
MP15 & V & 0.02 & $7 \mathrm{~d}$ & SVA & Plasma & Incomplete \\
MP16 & IX & 0.04 & $3 \mathrm{~d}$ & SVA & Plasma & yes \\
MP17 & V & 0.04 & $3 \mathrm{~d}$ & SVA & Plasma & yes \\
MP18 & XI & 0.02 & $1 \mathrm{~d}$ & SVA & Plasma & yes \\
MP19 & XI & 0.02 & $3 \mathrm{~d}$ & SVA & Plasma & yes \\
MP20 & XI & 0.04 & $1 \mathrm{~d}$ & SVA & Plasma & yes \\
MP21 & XI & 0.04 & $3 \mathrm{~d}$ & SVA & Plasma & yes \\
\hline
\end{tabular}

\section{Structure-Giving Workup}

The effect of different structure giving workups prior to template removal was investigated. A typical procedure is to place the films after deposition in a controlled humidity chamber $(50 \% \mathrm{RH})$. Here, solvent annealing was chosen as method due to the previously studied phase separation behavior of the multiblock copolymers (see section 2.5.2). It can be assumed that solvent annealing promotes the process of orientation of the mesostructured hybrid phase. The prepared films were placed in a solvent chamber and exposed to a solvent vapor mixture of methanol-THF for $2 \mathrm{~h}$. To control the effect of the solvent annealing, samples with no further structural giving procedure were prepared. In the end, all samples were treated with oxygen plasma to remove the template (see Figure 3.5). 


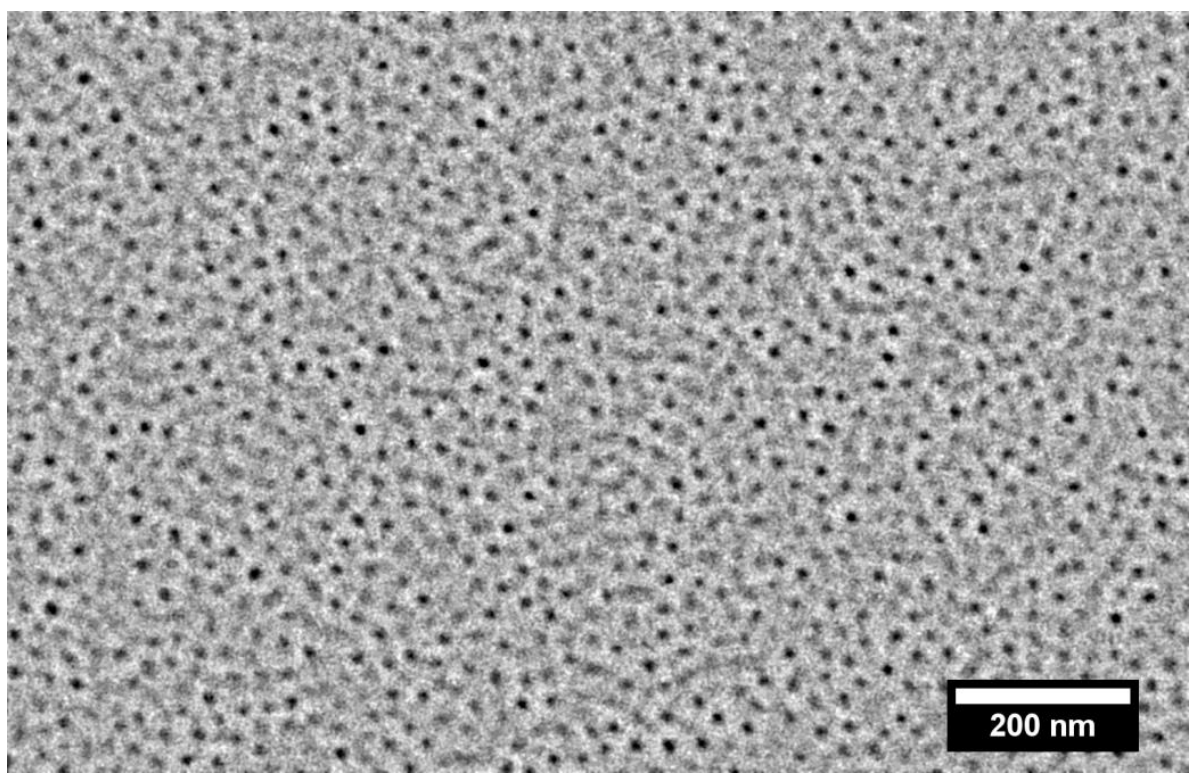

Figure 3.5. SEM micrograph of mesoporous silica thin film MP8 via solvent annealing using MeOH-THF 2:1 (v:v) after template removal by oxygen plasma treatment.

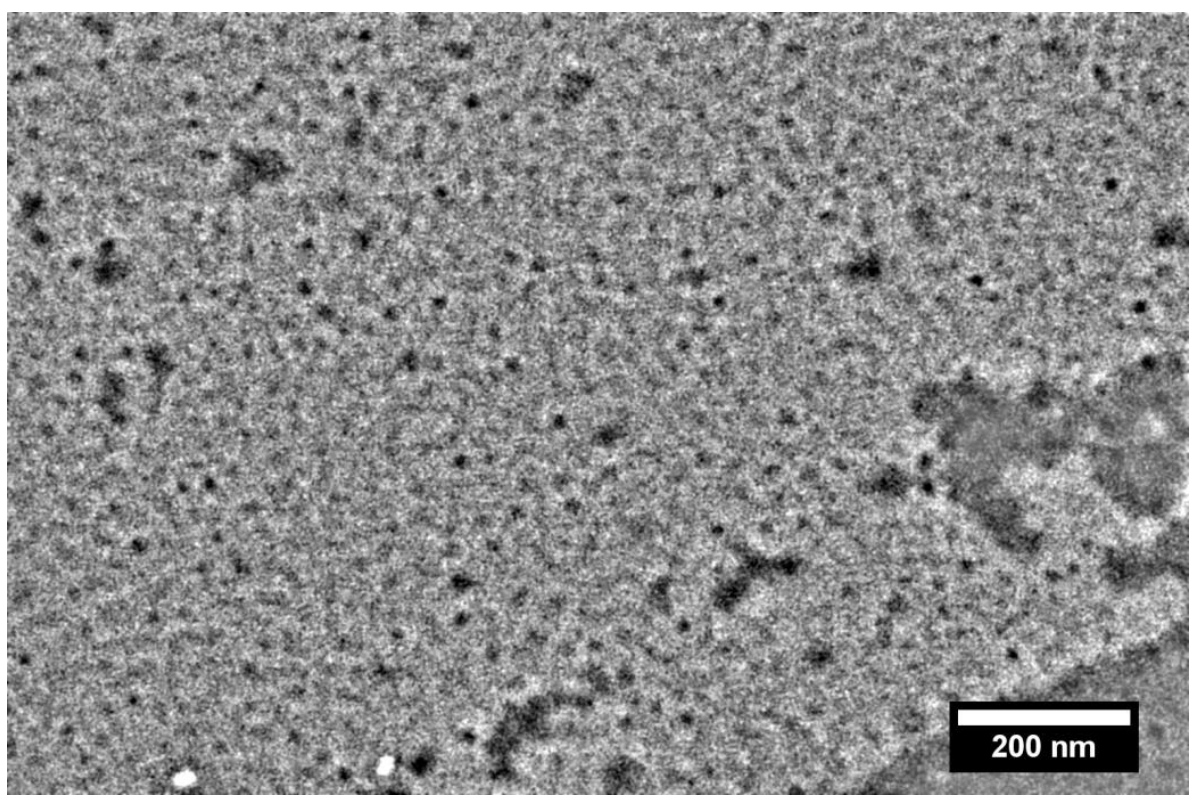

Figure 3.6. SEM micrograph of mesoporous silica thin film MP10 without any structural giving workup and template removal via oxygen plasma treatment. 
Representatively, the result of sample MP8 and MP10 will be briefly discussed. The samples were prepared with multiblock copolymer V. MP8 was solvent annealed using methanol THF 2:1 (v:v) for $2 \mathrm{~h}$ and. On sample MP10 no further treatment was applied before template removal. Figure 3.5 and Figure 3.6 show the SEM micrographs after template removal. The surface was analyzed at a minimum of three different areas in order to ensure the displayed structures were representative for the entire sample. As can be seen in Figure 3.6, no long-range order of the mesoporous material MP10 was observed. Some randomly distributed pores (darker areas) are visible. However, the solvent annealed sample appears to have pores over a wide range of the substrate surface. Surprisingly, the pores are not arranged in any ordered structure such as hexagonal. It cannot be excluded that the oxygen plasma procedure changes the previously arranged hexagonally cylinders by heating the sample during the etching process. This may have occurred because the structure was not examined prior to removal. In general, no pore structure was observed for the mesoporous silica thin films without any treatment in combination with plasma treatment, as shown in Table 3.2. For further investigations the samples were solvent annealed with a methanol-THF mixture in order to promote the mesostructure of the silica.

\section{Template Removal}

Commonly, the organic template is removed by calcination at temperatures between $300-500{ }^{\circ} \mathrm{C}$. In order to determine the appropriate calcination temperature thermogravimetric analysis (TGA) was performed. Exemplary one multiblock copolymer, V, was measured to obtain the degradation temperature. The measurement showed two steps of mass loss, visualized in Figure 3.7. The first drastic mass loss at $400{ }^{\circ} \mathrm{C}$ can be attributed to the degradation of PS segments and the second mass loss to PNIPAM. The slight decrease of mass at $\sim 100{ }^{\circ} \mathrm{C}$ results from residual solvent inside the polymer. This measurement revealed that temperatures above $600{ }^{\circ} \mathrm{C}$ are necessary to remove all organic template material. Such higher temperature would lead to sintering of the silica and thus to the loss of the desired porosity.[177] Due to this effect, $450{ }^{\circ} \mathrm{C}$ was maintained for $1 \mathrm{~h}$ and the resulting thin film is shown in Figure 3.8. 
3 Novel Mesoporous Silica Thin Films via Multiblock Copolymers

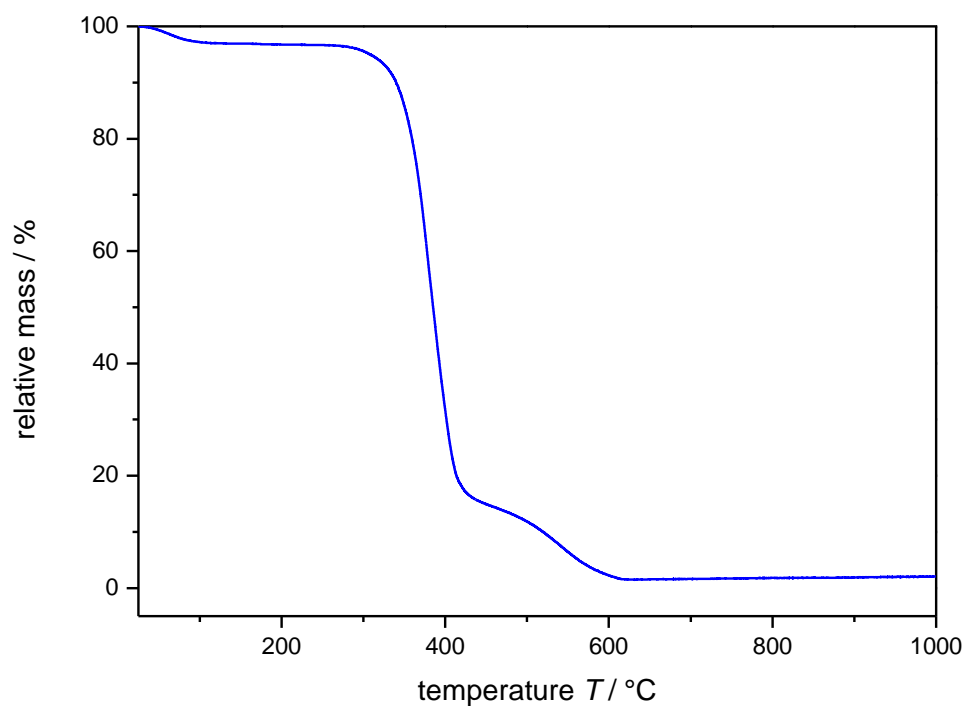

Figure 3.7. Thermogravimetric analysis of the multiblock copolymer V.

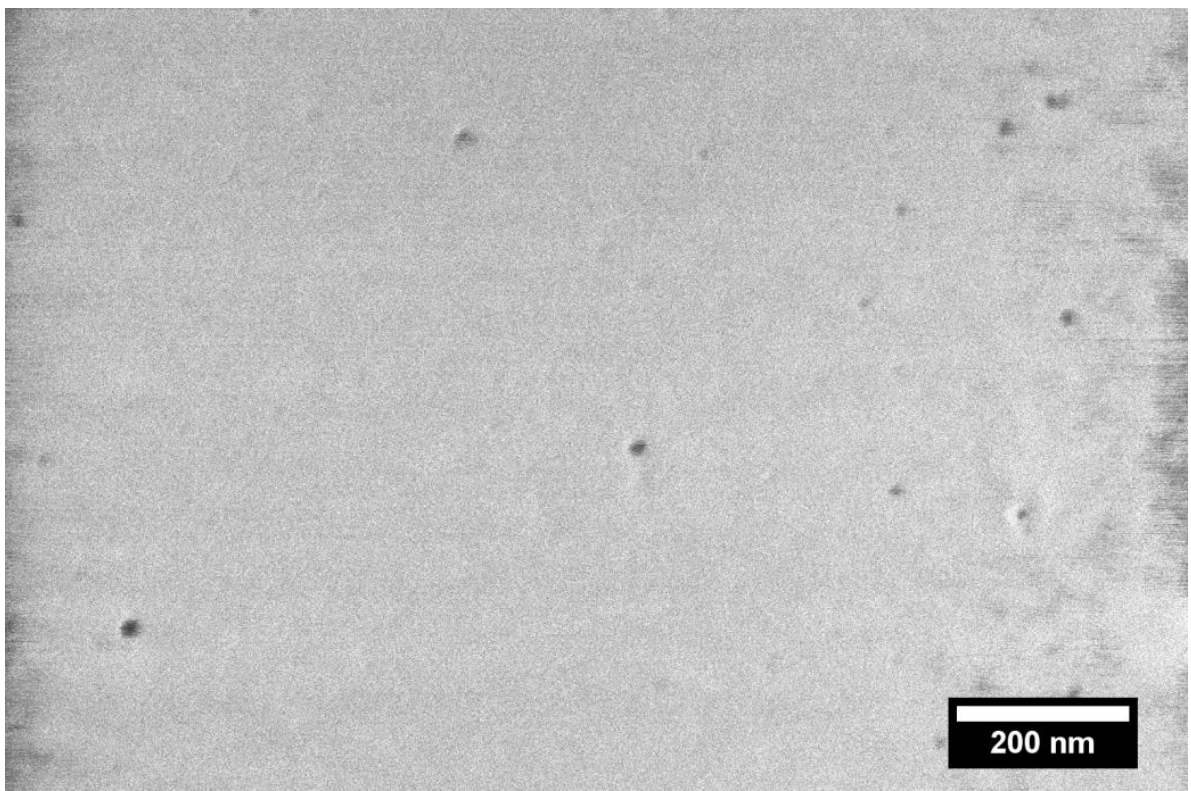

Figure 3.8. SEM-image of MP9 after template removal at $450{ }^{\circ} \mathrm{C}$ for $1 \mathrm{~h}$. 
In Figure 3.8, no mesoporous structured film is formed after thermal treatment at $450{ }^{\circ} \mathrm{C}$ for $1 \mathrm{~h}$. The absence of any pores indicates the ineffectiveness of this method to degrade all polymer material from the film. Alternatively, oxygen plasma etching can be applied in order to gain mesoporous materials. The oxygen plasma removes all organic matter that is present in the thin film. In literature, oxygen plasma has been demonstrated to selectively remove polymer from an inorganic-polymer hybrid film leading to well-organized structures.[178-180] Applying oxygen plasma as a template removal method resulted in a distinct mesoporous structure which is displayed in Figure 3.5. However, inconsistent results from other samples were observed. Some samples led to partially or fully mesoporous materials as can be seen in Appendix B. Due to the lower experimental effort, the oxygen plasma etching process was used to form mesoporous materials for the subsequent samples.

The formation of mesoporous silica films can be influenced by chemical parameters and preparation parameters. The different parameters such as sol aging time, amount of MBC and block number within the MBC will be varied in the following sections to highlight the influence on the formation of mesoporous structures.

\subsubsection{Influence of Sol Aging Time}

The effect of sol aging time on the organization of the mesoporous silica thin films will be analyzed. Thin films were prepared with the same sol composition using multiblock copolymer IX as template. The aging time of the final sol was varied between 1 (MP5), 3 (MP12) and 7 (MP14) days. In Figure 3.9, MP5 displays a porous structure. However, the pores are not arranged in any ordered structure. The pore size distribution was determined via Image from SEM images according to the method described in literature. ${ }^{[181,182]}$ An average pore diameter of $(12 \pm 2) \mathrm{nm}$ was calculated. Furthermore, the porosity can be estimated from the SEM images. The porosity $p$ is defined as:

$$
p=\frac{V_{\text {Pore }}}{V_{\text {total }}} \cdot 100
$$

Herein, $V_{\text {pore }}$ is the pore volume and $V_{\text {total }}$ is the total volume of the mesoporous film. To determine the porosity the equation can be modified assuming an equal height of the pores and the total film (see equation (3.2)).

$$
p=\frac{A_{\text {Pore }}}{A_{\text {total }}} \cdot 100 .
$$


The area of the pores $A_{\text {pore }}$ can be determined by ImageJ. For the area of the total film $A_{\text {total }}$ the image size of the examined surface is used. The resulting values are displayed in Table 3.3. All samples exhibited a rather low porosity in comparison to literature known mesoporous silica thin films. ${ }^{[175]}$ The porous structure of all samples were also verified via AFM. Figure 3.10 shows a representative image. By increasing the stirring time and thus the aging time of the solution the pore size did not change drastically but the porosity is increased by a factor of two (see Figure 3.11). Further prolonged aging time led to the disappearance of the porous structure (see Figure 3.12). The same effect was observed for the mesoporous silica film prepared with multiblock copolymer V (see Table 3.3). The best organization with moderate porosity was achieved after 3 days. After longer aging time the organized structure disappeared. Similar behavior for the preparation of mesoporous silica thin films has been observed in literature. ${ }^{[183,184]}$ The degree of condensation gets too high with increasing aging time and results in a higher viscosity of the solution. Therefore, the self-assembly of the organic template and inorganic species is hindered after the solvent and water are removed from the layer during the casting process. Here, the optimal aging time was found to be 3 days which is rather short compared to literature-known mesoporous silica thin films (7 days).[184]

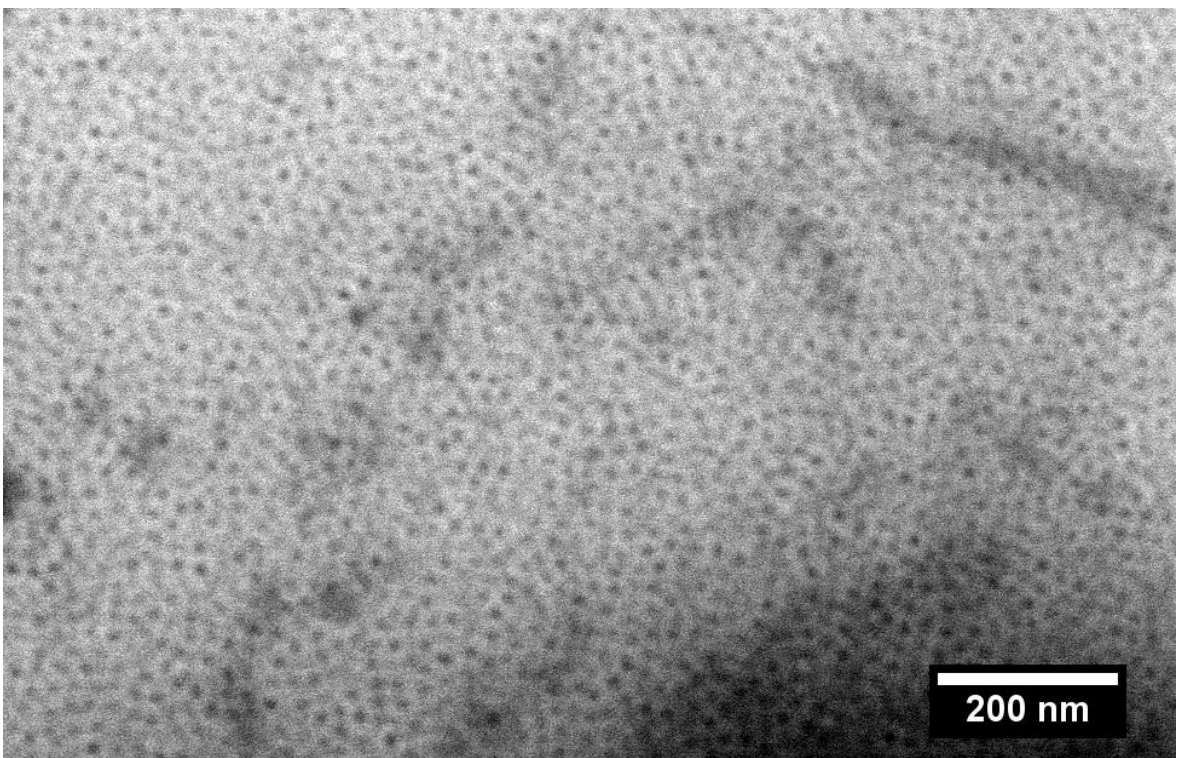

Figure 3.9. SEM image of MP5 after 1 day of sol aging. 

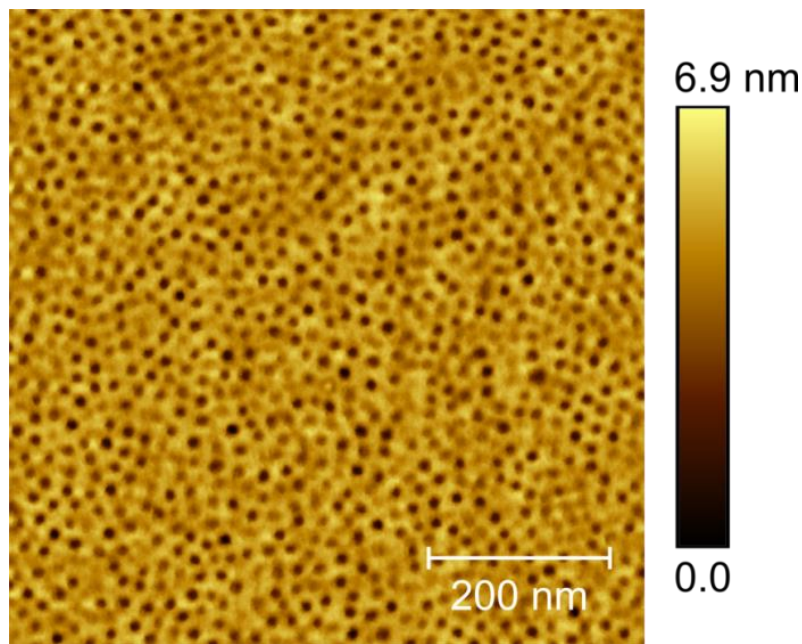

Figure 3.10. AFM topography image of sample MP5.



Figure 3.11. SEM image of MP12 after 3 day of sol aging. 


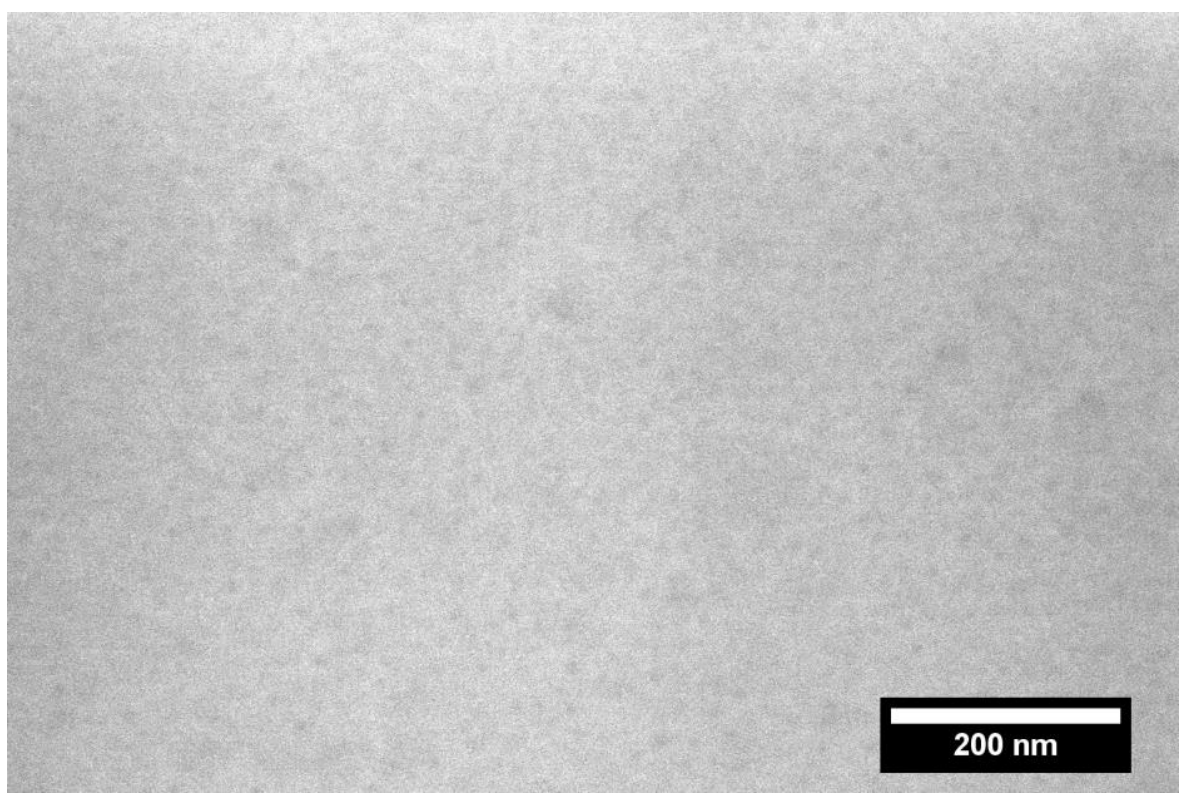

Figure 3.12. SEM image of MP14 after 7 day of sol aging.

Table 3.3. Final stirring times of the prepared sols with multiblock copolymers (MBC), determined film thickness by ellipsometry, average pore size diameter and porosity from SEM images via ImageJ.

\begin{tabular}{cccccc}
\hline sample & MBC & $\begin{array}{c}\text { final } \\
\text { stirring } \\
\text { time / d }\end{array}$ & $\begin{array}{c}\text { film } \\
\text { thickness / } \\
\mathbf{n m}\end{array}$ & $\begin{array}{c}\text { average pore size } \\
\text { diameter (SEM) } \\
\boldsymbol{d}_{\text {pore }} / \mathbf{n m}\end{array}$ & $\begin{array}{c}\text { porosity } \\
\boldsymbol{p} / \mathbf{\%}\end{array}$ \\
\hline MP5 & IX & 1 & $20.5 \pm 0.3$ & $12 \pm 2$ & 9.9 \\
MP12 & IX & 3 & $23.6 \pm 0.3$ & $11 \pm 2$ & 18.6 \\
MP14 & IX & 7 & $18.0 \pm 0.3$ & - & - \\
\hline MP8 & V & 1 & $23.1 \pm 0.3$ & $13 \pm 2$ & 9.8 \\
MP13 & V & 3 & $24.3 \pm 0.3$ & $14 \pm 3$ & 19.6 \\
MP15 & V & 7 & $22.6 \pm 0.3$ & - & - \\
\hline
\end{tabular}

Furthermore, nitrogen adsorption-desorption measurements were performed and the isotherms are displayed in Figure 3.13. All samples show a hysteresis in the isotherms, indicating capillary condensation and a mesoporous structure with interconnected channels. According to the classification of IUPAC (see section 3.2), type IV isotherms with a hysteresis loop are observed.[185] The characteristic hysteresis of this isotherm is associated with capillary condensation and evaporation taking place in the mesopores. The initial part of the isotherm can be assigned to monolayer- 
multilayer adsorption on the walls which is followed by pore condensation. A type $\mathrm{H} 3$ hysteresis loop is observed and the capillary condensation occurs at higher relative pressure $\left(P / P_{0} \sim 0.8\right)$. This $\mathrm{H} 3$ type is usually observed for plate-like particles or slit-shaped pores. Considering the relatively low film thickness of the samples (see Table 3.3) only bilayer or trilayers of silica were formed which could explain the plate-like behavior. The film thickness was determined via ellipsometry using the Brüggemann effective medium approximation (BEMA). ${ }^{[186]}$ The model is considers a two phase layer made of silica with spherical void pores. The calculated porosity was used as pore volume fraction and optical data for silica framework and air for the void pores were applied.

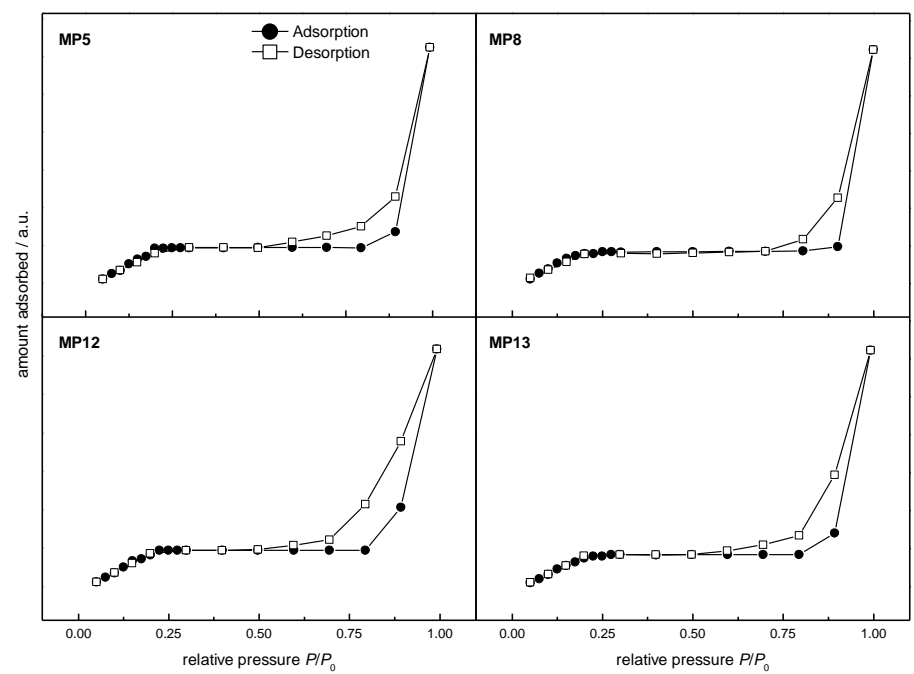

Figure 3.13. Nitrogen adsorption-desorption isotherms type IV with hysteresis loop H3 for sample MP5, MP8, MP12 and MP13.

\subsubsection{Influence of the Amount of MBC}

The formation and organization of mesoporous silica films also depends on the sol composition. ${ }^{[184]}$ To investigate this effect the amount of MBC in the solution was varied from $0.02 \mathrm{~g}$ to $0.04 \mathrm{~g}$ (see Table 3.4). Thin films with MBC V and IX were prepared and the corresponding SEM images are shown in Figure 3.14 and Figure 3.15. The SEM images of sample MP16 and MP17 show that the degree of organization appears to be higher compared 
to the structures that were observed when the lower amount of MBC was used. However, comparable adsorption-desorption properties were found and the pore size and porosity stayed constant with increasing amounts of MBC. All samples exhibited type IV with a type H3 hysteresis loop (as described above). The isotherms are displayed in the Appendix B. Such concentration effect of the template has been observed in previous works for the preparation of mesoporous silica thin films.[187] The alignment is enhanced due to the extended self-assembly of the organic/inorganic species. The higher amount of MBC promotes the micelle formation in early stages of the coating and drying procedure and thus the time of selfassembly is increased. In sols with lower amounts of MBC, the formation of micelle formation is slowed. Therefore, the duration of self-assembly is reduced leading to poorly formed mesostructures.

Table 3.4. Amount of the MBC within in the prepared sols, determined film thickness by ellipsometry, average pore size diameter and porosity from SEM images via ImageJ.

\begin{tabular}{lccccc}
\hline sample & MBC & $\begin{array}{c}\text { amount of } \\
\text { MBC/g }\end{array}$ & $\begin{array}{c}\text { film } \\
\text { thickness / } \\
\mathbf{n m}\end{array}$ & $\begin{array}{c}\text { average pore size } \\
\text { diameter (SEM) } \\
\boldsymbol{d}_{\text {pore }} / \mathbf{n m}\end{array}$ & $\begin{array}{c}\text { porosity } \\
\boldsymbol{p} / \mathbf{\%}\end{array}$ \\
\hline MP12 & IX & 0.02 & $23.6 \pm 0.3$ & $11 \pm 2$ & 18.6 \\
MP16 & IX & 0.04 & $23.7 \pm 0.3$ & $13 \pm 1$ & 19.0 \\
\hline MP13 & V & 0.02 & $24.3 \pm 0.3$ & $14 \pm 3$ & 19.6 \\
MP17 & V & 0.04 & $25.5 \pm 0.3$ & $15 \pm 3$ & 19.8 \\
\hline
\end{tabular}



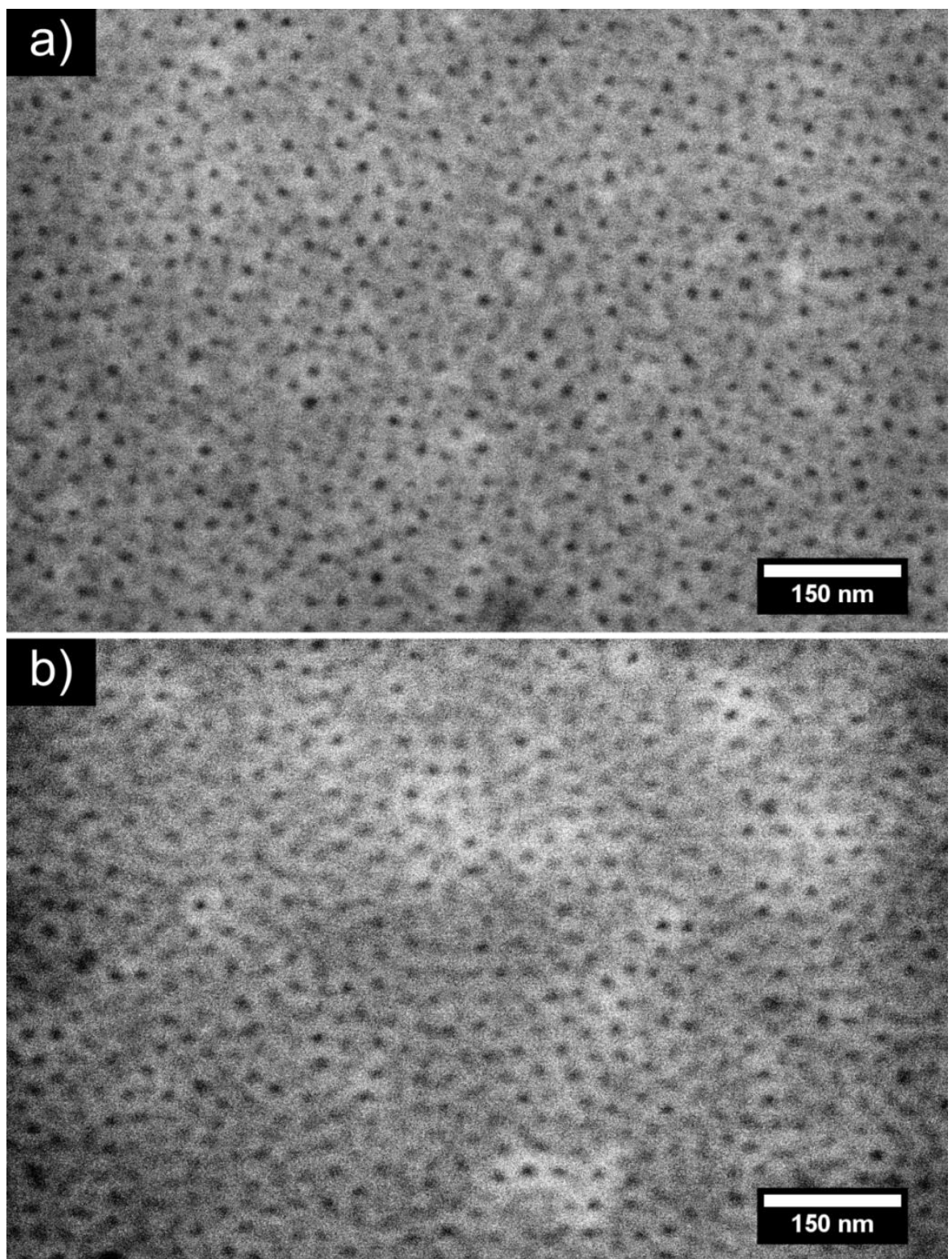

Figure 3.14. SEM image of a) MP13 and b) MP17. 

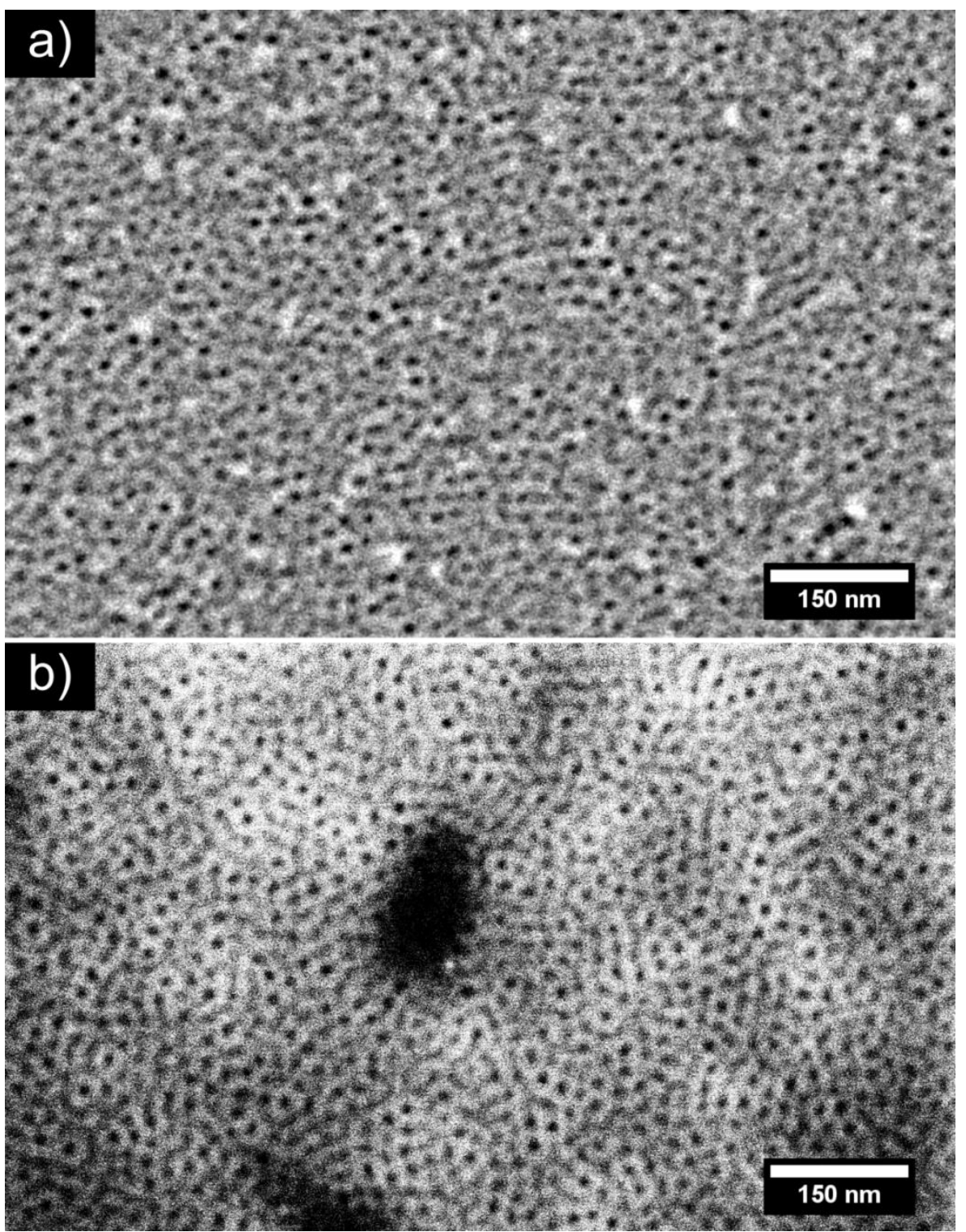

Figure 3.15. SEM image of a) MP12 and b) MP16. 


\subsubsection{Influence of the Chemical Nature of the MBC upon Mesostructure Formation}

So far, the influence of the preparation procedure on the film formation was investigated leading to an optimal sol aging period of 3 days and $0.04 \mathrm{~g}$ as a deployed amount of MBC. Here, the chemical nature of the utilized $\mathrm{MBC}$ on the formation of mesostructures will be examined.

First, the segment number within in the MBC is varied (see Table 3.5). In section 2.5.2 it was demonstrated that $\mathrm{MBC}$ with volume fractions between $0.59<f_{\text {PNIPAM }}<0.77$ arrange into hexagonally packed cylinders perpendicular to the substrate, by solvent annealing, independent of the segment number. MBC V, IX and XI are used which exhibit roughly the same volume fraction of PNIPAM but differ in the number of segments. The mesoporous silica thin films were prepared as described above and the resulting structures are shown in and Figure 3.14b, Figure 3.15b and Figure 3.16. Two different effects were observed. First, for sample MP21 compared to MP16 and M17 larger pores with an average pore size of $(25 \pm 8) \mathrm{nm}$ were achieved. Similar pore sizes were observed with shorter aging time and lower MBC content using MBC XI. Nevertheless, no clear trend is observed when the segment number is increased which can be attributed to the difference of molar mass. In addition, porosity is slightly lower for sample MP21.

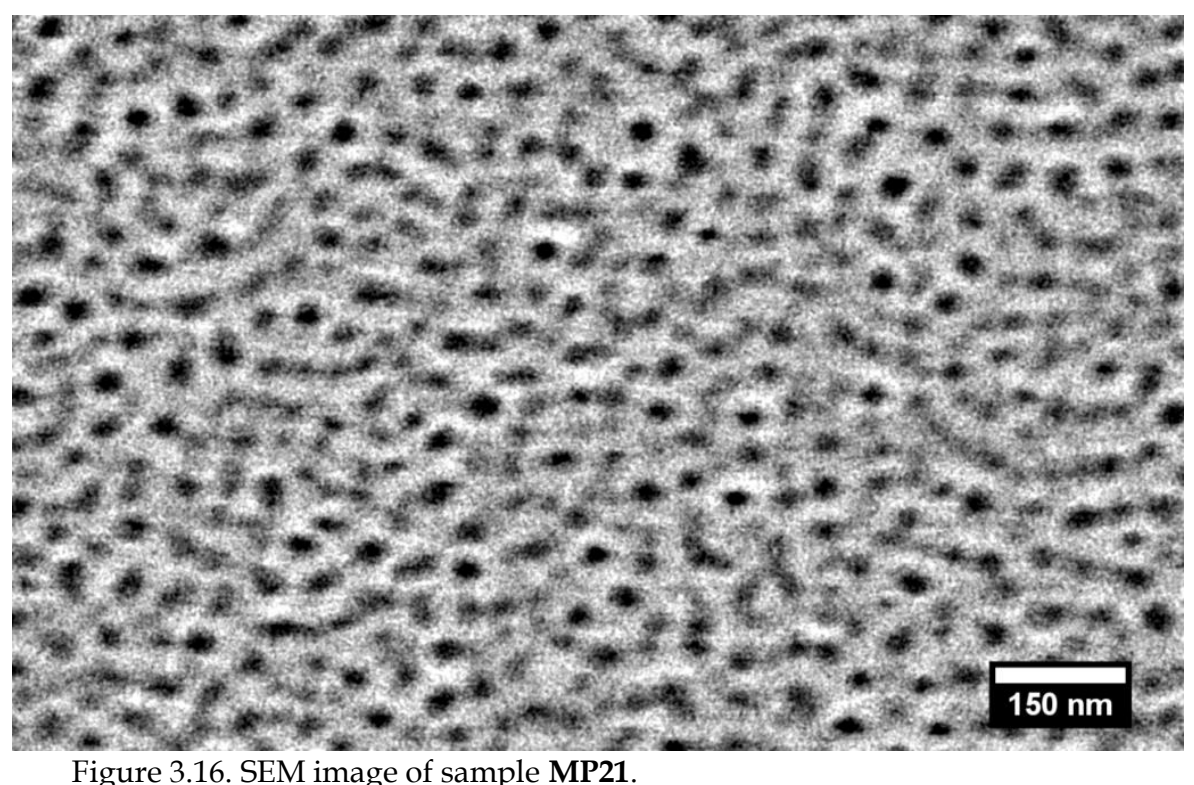


Secondly, the degree of organization is enhanced in the system using MBC with segment number $s>3$. Again, all samples exhibited type IV with a type $\mathrm{H} 3$ hysteresis loop. It is reasonable that the segment number within the MBC has a strong effect upon the pore formation. The difference in the pore size upon the segment number might be explained by the chain formation in the solvent. In general, block copolymers aggregate into spherical micelles in selective solvents with a concentration higher than the cmc. Halperin et al. demonstrated that linear $(\mathrm{AB})_{\mathrm{n}}$-multiblock copolymer arrange into flower-like single intramolecular micelle or linear string of molecular micelles in selective solvents depending on the repeating number $\mathrm{n}$ of the AB block (see Figure 3.17). ${ }^{[188,189]}$ MBC X or XI exhibit three segment (which correlate to $n=1.5$ ) and therefore, the formation of a single intramolecular micelle is reasonable in the sol-gel solution. On the contrary, MBC V and IX contain a higher segment number in the polymer and thus the formation of a linear string of molecular micelles is more likely.

a)

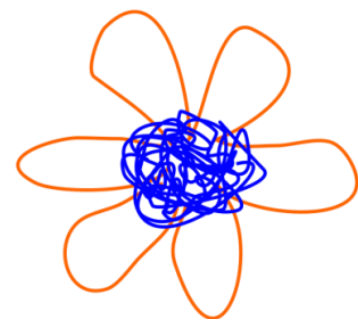

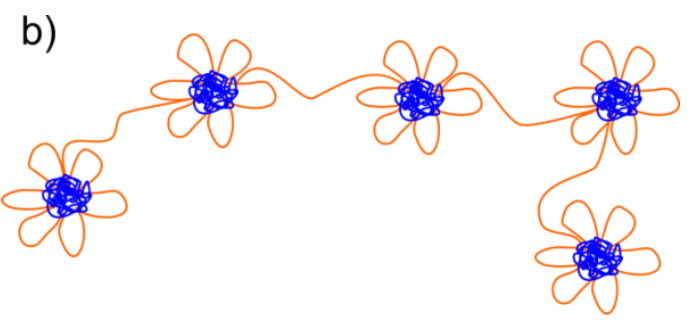

Figure 3.17. Schematic illustration of micelle morphologies of linear multiblock copolymers a) flower-like single intramolecular micelle and b) linear string of molecular micelles.

Table 3.5. Characteristics of mesoporous silica thin films using MBC with varied segment number $s$. Film thickness was monitored by ellipsometry, average pore size diameter and porosity were determined from SEM images via ImageJ.

\begin{tabular}{cccccccc}
\hline sample & MBC & $\begin{array}{c}\overline{\boldsymbol{M}}_{\mathrm{n}, \mathbf{0}} / \\
\mathbf{g} \cdot \mathbf{m o l}-1\end{array}$ & $f_{\text {PNIPAM }}$ & $\boldsymbol{s}$ & $\begin{array}{c}\text { film } \\
\text { thickness } \\
\mathbf{n m}\end{array}$ & $\begin{array}{c}\boldsymbol{d}_{\text {pore }} / \\
\mathbf{n m}\end{array}$ & $\begin{array}{c}\text { porosity } \\
\boldsymbol{p} / \boldsymbol{\%}\end{array}$ \\
\hline MP16 & IX & 54600 & 0.74 & 7 & $23.7 \pm 0.3$ & $13 \pm 1$ & 19.0 \\
MP17 & V & 127900 & 0.77 & 9 & $25.5 \pm 0.3$ & $15 \pm 3$ & 19.8 \\
MP21 & XI & 64500 & 0.70 & 3 & $33.7 \pm 0.5$ & $25 \pm 8$ & 17.9 \\
\hline MP1 & X & 52400 & 0.54 & 3 & $25.1 \pm 0.3$ & $22 \pm 5$ & 22.8 \\
MP18 & XI & 64500 & 0.70 & 3 & $33.0 \pm 0.5$ & $24 \pm 6$ & 16.6 \\
\hline
\end{tabular}

Mesoporous silica thin films using MBC $\mathbf{X}$ were prepared. MBC $\mathbf{X}$ and XI exhibit three segments but differ in the composition. Yu et al. 
demonstrated that by increasing the hydrophobic volume the pore size is increased due to the greater size of the hydrophobic micelle core. ${ }^{[190]}$ But in contrast to these earlier findings no significant difference in the pore size was observed by decreasing the hydrophobic volume fraction of PS from 0.46 to 0.30 . The SEM image of sample MP1 (Figure 3.18a), using MBC $\mathbf{X}$ as template, showed an average pore size of (22 \pm 5$) \mathrm{nm}$. MP18 exhibits an average pore size of $(24 \pm 6) \mathrm{nm}$. However, careful attention must be exercised in these results since only two samples are compared to each other. Further experiments must be performed to exclude any other influences. 


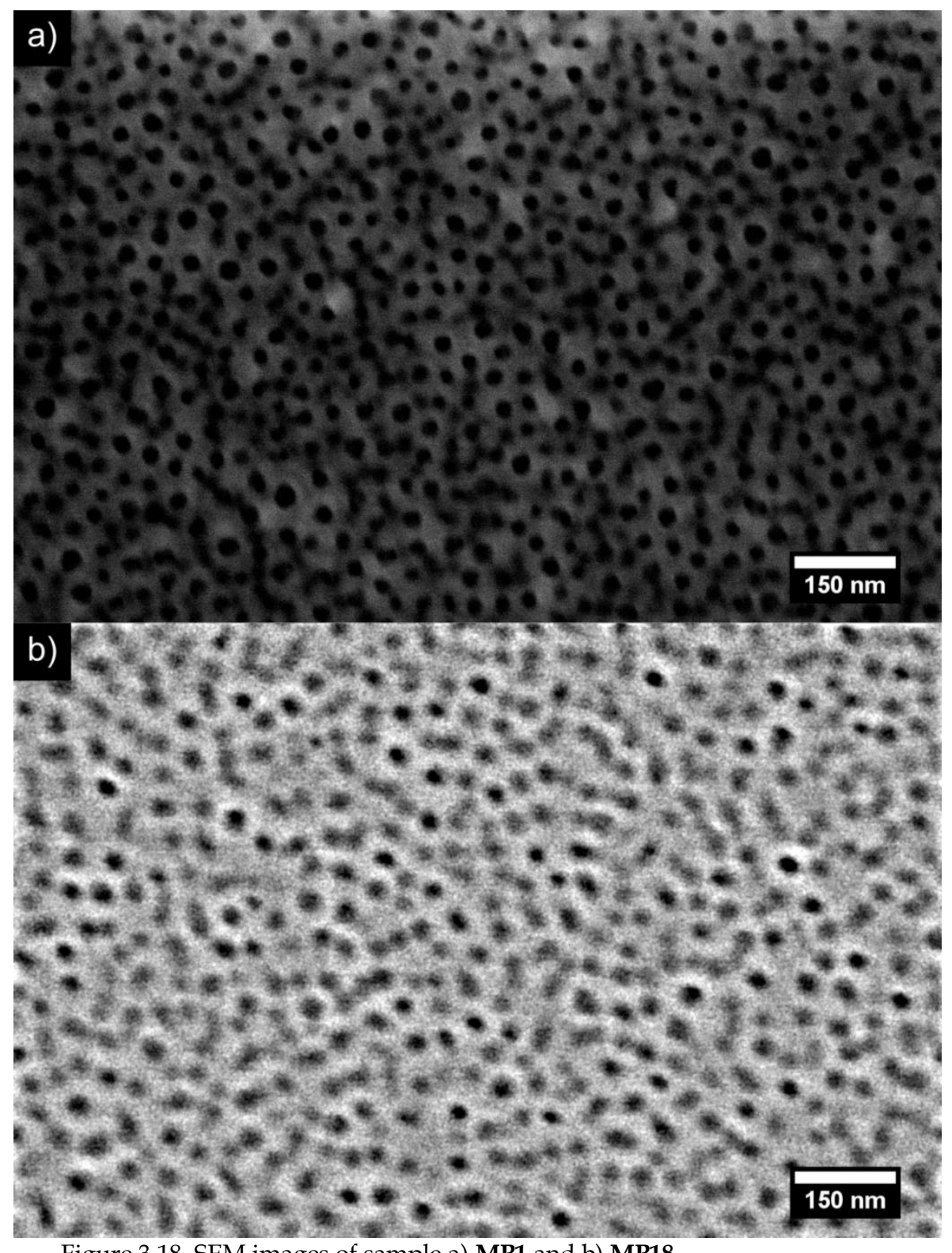

Figure 3.18. SEM images of sample a) MP1 and b) MP18. 


\subsection{Conclusions}

In summary, a facile technique to prepare mesoporous silica thin film was systematically developed by the EISA method using a combination of sol-gel and amphiphilic multiblock copolymers as templates. The previously well-studied multiblock copolymers contained PS as the hydrophobic segment and PNIPAM as the hydrophilic segment. They were employed as structure directing agents due to their ability to self-assemble in microdomains on the nanometer scale. Here, the combination of sol-gel chemistry, polycondensation of silicate species, and organization of amphiphilic templates led to a mesoporous structure with a large pore size (11-27 nm). Solvent annealing was applied to arrange the multiblock copolymers and thus the incorporated silicate species. The prepared thin films were characterized by techniques such as SEM, AFM, ellipsometry and nitrogen adsorption-desorption measurements. The pores size and porosity were directly accessible by SEM and AFM. Ellipsometry revealed film thickness below $35 \mathrm{~nm}$. Furthermore, nitrogen adsorption-desorption measurements confirmed the interconnectivity of the pores and suggest a split-like or particle-like shape of the mesoporous silica thin films.

In order to remove the organic template and gain the mesoporous silica network oxygen plasma treatment was ruled out as an efficient method. The effect of different preparation parameters on the thin film formation was systematically studied. Sol aging time and amount of employed multiblock copolymer were found to be important factors for the formation of the mesoporous silica thin film. An optimal sol aging time of 3 days seemed to exist for the silicate species resulting in moderate porosity. Longer aging times led to higher condensation degree of the silicate and loss of the mesoporous structure upon template removal. In addition, a suitable amount of the multiblock copolymer utilized in acid-catalyst TEOS solution was determined. Higher amounts promoted the self-assembly of the organic/inorganic interface and showed higher organization of the pores. As another import factor, it was revealed that the nature of the multiblock copolymer has an influence on the pore size due to their collapsed state in selective solvents. Multiblock copolymers exhibiting segment numbers $s \leq 3$ achieve a pore size greater than $16 \mathrm{~nm}$ and higher segment numbers $s>3$ lead to well-formed pores of size $\leq 15 \mathrm{~nm}$. In contrast, no influence of the polymer composition was observed upon the pore size. 


\section{Thermosensitive Cation-Selective Mesochannels ${ }^{2}$}

\subsection{Preface}

The possibility of creating fully synthetic hybrid assemblies has been one of the grand challenges for materials science in the 21st century.[14,191-194] Mimicking the gating and charge selective properties which are observable in biological channels has been of special interest. In this context, mesoporous materials were demonstrated to be a robust alternative for creating nanoscopicc channels as they offer exquisite control over the pore characteristics. In addition, they are compatible with functional systems.[110,157,195-197]

Mesoporous nanoarchitectures are appealing due to their ability to incorporate macromolecules which can act as a gate and allow for the entry or release of chemical species into or from the mesoporous matrix.[198-206] The species can be entrapped in the inner pores or the latter can be emptied. The gate opens upon application of an external stimulus and the hybrid material either releases the confined guests or permits the entrance of molecular species from the bulk solution.[207-213] During the last decade, significant research efforts have been focused on the quest for novel alternative switchable nanopore machineries that are capable of nanoactuating by external stimuli in a controllable manner.[214-221] In this regard, one stimulus of particular interest in biological systems is temperature. ${ }^{[222-225]}$ Biological ionic channels that are activated by changing the temperature transduce this information into conformational changes that open the channel pore. A typical example is thermosensation, which is carried out by the direct activation of thermally gated ion channels within the surface membranes of sensory neurons.[226] This complex task is accomplished by temperature sensitive, cation-selective channels that are members of the extensive TRP family (Transient Receptor Potential

\footnotetext{
${ }^{2}$ Reproduced with permission from S. Schmidt, S. Alberti, P. Vana, G. J. A. A. Soler-Illia, O. Azzaroni: Thermosensitive Cation-Selective Mesochannels: PNIPAM-Capped Mesoporous Thin Films as Bioinspired Interfacial Architectures with Concerted Functions, Chemistry - A European Journal, 2017, 23, 14500-14506, Copyright Wiley-VCH Verlag GmbH \& Co. KGaA.
} 
channels).[227] These biological entities act as gateable ionic filters enabling the selective passage of cations only under specific thermal conditions. ${ }^{[228]}$ It has been demonstrated that this gating process is achieved through concerted functions of hydrophobic and charged residues in the biological channel.[229,230] The artificial design of biological pores such as TRPs remains a challenge in molecular materials science. The design demands a robust and fully artificial interfacial architecture which displays charge selectivity with thermo-activated gating properties. In this context, the quest for concepts to create novel charge-selective membranes is of critical importance to further expand the scope of applications of these materials.

The integration of thermoresponsive poly( $N$-isopropylacrylamide) (PNIPAM) in mesoporous matrices has been the subject of study of different groups.[231-236] PNIPAM is a widely studied polymer, mainly because of its lower critical solution temperature (LCST) behavior in water, as it precipitates upon heating above $T \approx 31^{\circ} \mathrm{C} .[237,238]$ This temperatureinduced phase separation has been exploited for the construction of thermoassociative polymeric systems.

The first example of mesoporous materials with integrated thermoactive gating properties was reported by Lopez and his collaborators in 2003.[239,240] Their approach is based on surface-initiated atom transfer radical polymerization of PNIPAM brushes grafted from mesoporous silica particles and it demonstrated that these macromolecular entities controlled the uptake and release of rhodamine 6G from the mesoporous particles. Afterwards, Oupicky et al. showed that densely-grafted PNIPAM-modified mesoporous silica nanoparticles exhibit good uptake and release properties of fluorescein at room temperature (below LCST) and a low level of leakage above LCST.[241] According to these authors, the densely grafted PNIPAM chains constitute the capping layer that prevents the uptake and release of cargo molecules when the polymer brushes are collapsed on the nanopore outlets. Since then, different groups explored similar concepts to thermally control the transport of chemical species through mesoporous matrices. However, the possibility of creating thermoactuated cation-selective mesoporous platforms remains fully unexplored.

Surface-initiated RDRP techniques are often used to create hybrid organic-inorganic assembly. In this work, the surface-initiated RAFT polymerization was chosen to tether PNIPAM brushes to the mesoporous silica thin film. This polymerization technique is known to be a versatile method to functionalize surfaces. Several studies on the modification of silicon wafers, silica and titanium nanoparticles were performed.[242-250] The modified mesoporous silica thin films were characterized via water contact angle, X-ray photoelectron spectroscopy (XPS) and AFM, environmental ellipsometric porosimetry (EEP) and cyclic voltammetry. Furthermore, the 
polymer chains were analyzed by SEC and dynamic light scattering (DLS) measurements.

\subsection{Fundamentals of RAFT Polymerization at Surfaces}

Radical polymerization at surfaces such as silica particles, carbon nanotubes or mesoporous silica thin films are subdivided into three different approaches: grafting-through, grafting-to and grafting-from. Depending on the surface a suitable technique can be selected for tethering polymer chains to it. Mostly in the context of RAFT polymerization the grafting-to and grafting-from approach are used. In the grafting-through approach, monomer or polymerizable groups are immobilized on the surface (see Scheme 4.1, left). During polymerization, propagating chains from the solution can react with surface-bound monomers resulting in polymer chains that are directly anchored to the surface. A side-effect of this method is that, in addition to adding monomers from solution, the surfacebound macroradicals can react with surface-bound monomers resulting in loops.[251]

As part of the grafting-to approach, preformed polymers are immobilized on the surface (see Scheme 4.1, middle). The immobilization can be realized via formation of a covalent bond between polymer and surface or by physisorption of parts of the polymer. ${ }^{[252-254]}$ The advantage is the control of molar mass and dispersity since the polymers are prepared prior to immobilization. The disadvantage of this technique is the steric hindrance of the polymer chains that lead to very low grafting densities compared to the grafting-through approach. ${ }^{[255]}$ Nevertheless, using RAFT polymerization the grafting-to approach is a suitable method for the functionalization of gold surfaces. The thiocarbonylthio groups exhibit the inherent benefit of a high binding capacity to gold. ${ }^{[7]}$ In contrast, silica particles or mesoporous silica are mostly functionalized via the graftingfrom approach. ${ }^{[256]}$ In the grafting-to approach, initiators or control agents (e.g. RAFT agents) are covalently bound to the surface. As polymerization proceeds polymer chains grow from the surface. Using this approach, higher grafting densities are achievable compared to the grafting-to approach. However, the polymer chains need to be detached from the surface in order to characterize them. ${ }^{[255]}$ However, a disadvantage to this approach is poor control over the molar mass. [257] 

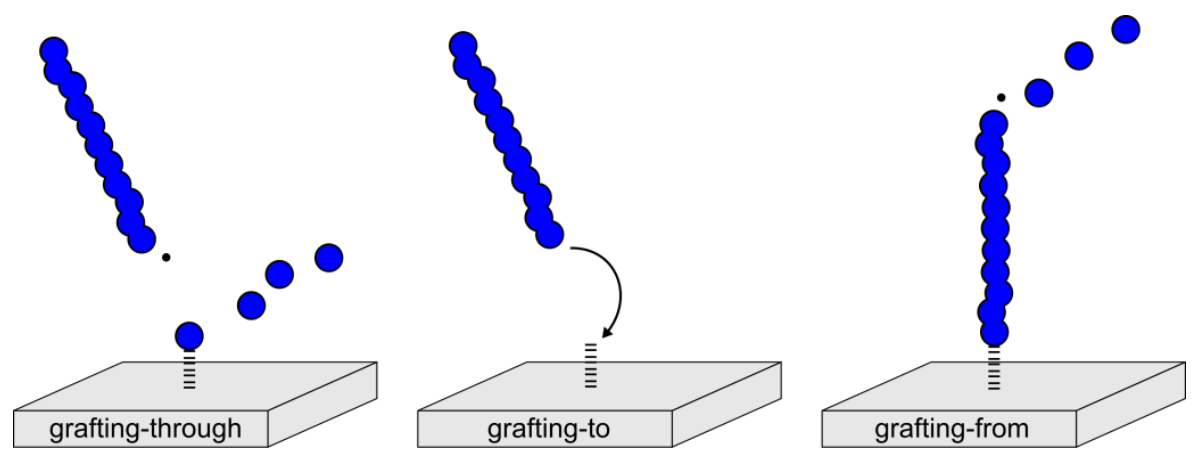

Scheme 4.1. Schematic illustration of grafting polymers to surfaces using different techniques.

In general, the immobilization of a RAFT agent can be achieved via its R- or Z-group (see Scheme 4.2, left). ${ }^{[255]}$ If the RAFT agent is anchored by its R-group, the polymer chains grow from the surface into the solution. The RAFT group extends into the solution and is therefore easily accessible to other macroradicals.[242]

In the Z-group approach, the RAFT agent is bound to the surface via its stabilizing Z-group (see Scheme 4.2, right). As shown in Scheme 4.2 the RAFT group is always located at the surface and the polymer chains only grow in the solution. The growing polymer chains are constantly added to and released back to the solution. Since the radical species diffuse to the surface during polymerization and then react with the RAFT group to generate polymer chains, this method is also referred to as the grafting-to approach. ${ }^{2255]}$ Due to this chain exchange mechanism between solution and surface, the anchored polymers are similar to the ones remaining in solution after completing the reaction. However, RAFT groups might get shielded by growing polymer chains and therefore diffusion of the radical species to the RAFT group becomes increasingly difficult. In general, sacrificial RAFT agent is often added to the reaction solution to ensure control of the polymerization and facilitate the polymer characterization. ${ }^{255,258]}$ 

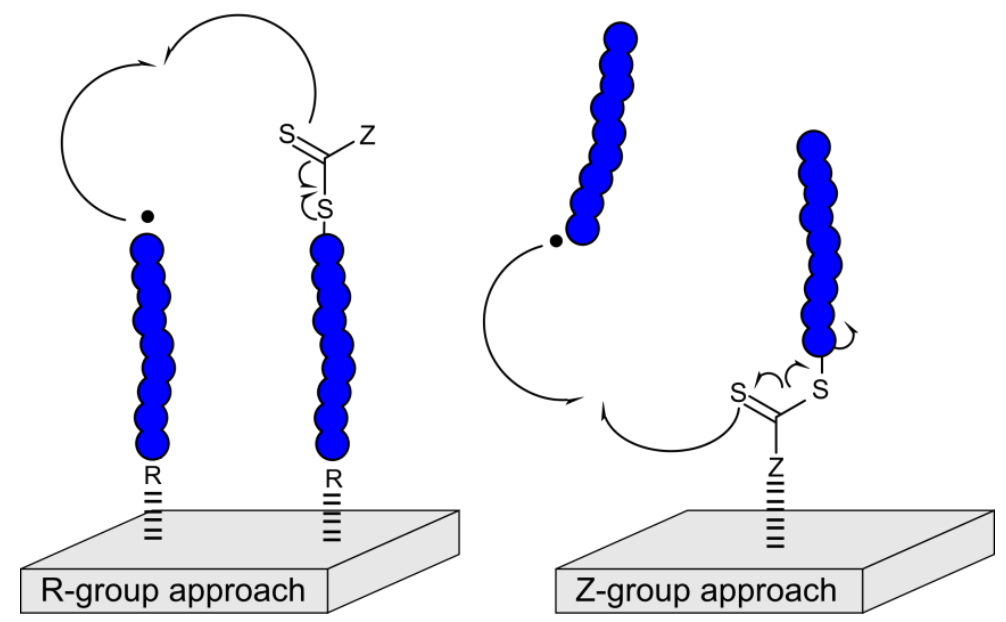

Scheme 4.2. Schematic illustration of the surface-initiated RAFT polymerization for the R- and Z-group approach.

\subsection{Synthesis of Thermo-Responsive Mesoporous Silica Thin Films}

This work describes the creation of a hybrid organic-inorganic assembly that displays thermo-dependent ionic transport properties. Until now this combination was not observed in nanoporous permselective membranes. In close resemblance to TRPs, functional hybrids constituted of PNIPAMcapped mesoporous silica-based thin films are able to discriminate and modulate the transport of cations by changing the temperature. In contrast, passage of anions is inhibited independent of the temperature. Being able to design multicomponent mesoarchitectures at interfaces with functions similar to thermosensitive biological channels is of great importance for further expanding the application of these designed nanoarchitectures.

Modification of the mesoporous films with PNIPAM brushes was accomplished by surface-initiated RAFT (SI-RAFT) polymerization (see Scheme 4.3). The mesoporous silica thin films were prepared via a one-pot sol-gel method by dip coating onto glass and indium tin oxide (ITO) substrates, following previously reported procedures. ${ }^{[155,259,260]}$ Mesoporous films with a thickness of about $200 \mathrm{~nm}$ were obtained. The measurements also showed highly accessible organized pore arrays (see Figure 4.1). Synchrotron-based SAXS characterizations were performed which confirmed these findings. The mesostructure presented a cubic $\operatorname{Im} \overline{3} m$ 
arrangement with an interpore distance of $12 \mathrm{~nm}$ (see Figure 4.1). Mesoporous surfaces were derived with a RAFT agent, benzyl(3(trimethoxysilyl)-propyl)trithiocarbonate (BT3 $\left.{ }^{3} \mathrm{PT}\right)$ via its Z-group which included a trifunctional anchor (see Scheme 4.3). The chosen RAFT agent has been the subject of a preceding study in which it was shown to be suitable to control a radical RAFT polymerization of NIPAM on silica particles.[261]
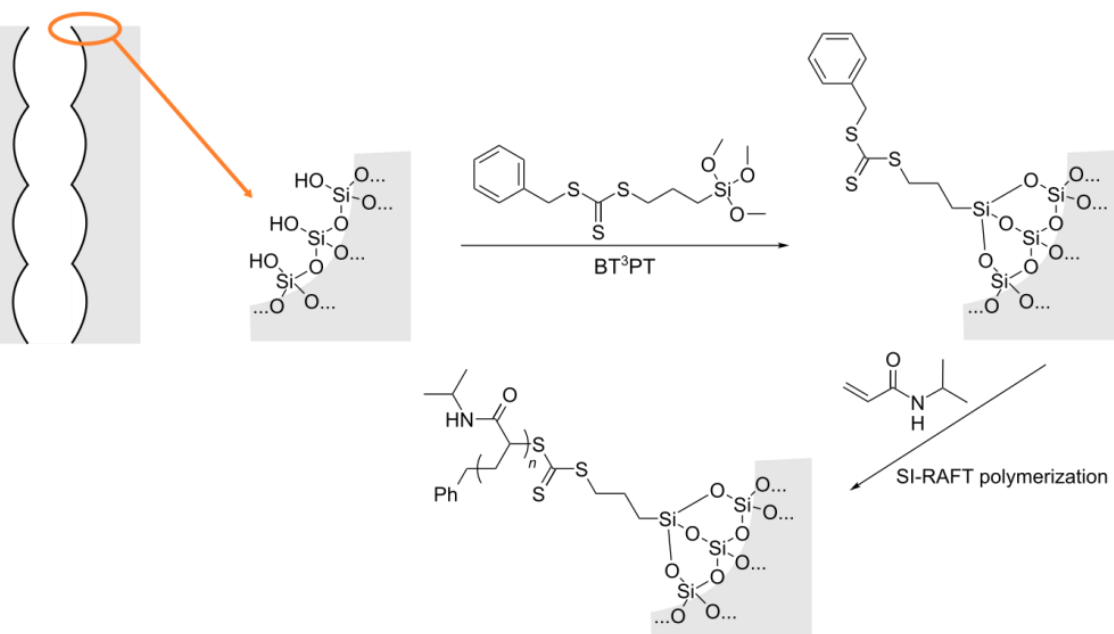

Scheme 4.3. Functionalization of the mesoporous silica thin films with SI-RAFT using BT ${ }^{3} \mathrm{PT}$ as RAFT agent.
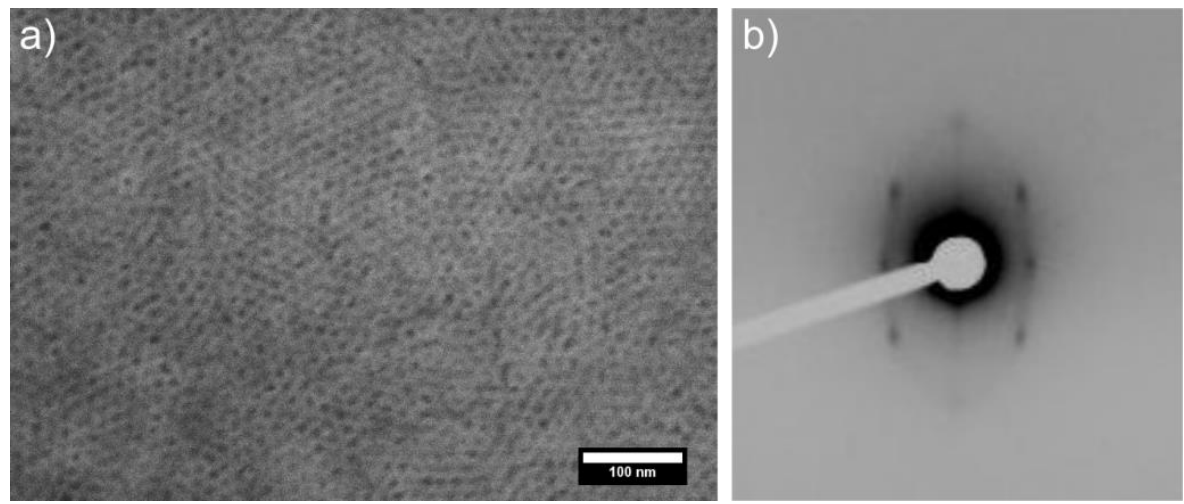

Figure 4.1. SEM image a) and SAXS-2D pattern b) of as-obtained mesoporous silica thin films. 
The remaining polymer in solution was characterized via SEC and DLS. Since the Z-group approach was employed, the surface-tethered polymers were assumed to have the same characteristics of the polymers in solution. The free polymer exhibits a molar mass of $44000 \mathrm{~g} \cdot \mathrm{mol}^{-1}$ and a dispersity of 1.33. Temperature responsiveness was assessed for the free polymer in water through DLS, measuring the change of the average diameter as a function of the temperature (see Figure 4.2). Below the LCST the PNIPAM chains are in the random coil configuration exhibiting hydrogen bonding between the polymer chains and water molecules. Increasing the temperature to a point above the LCST, water is expelled by replacing the intermolecular hydrogen bonds with intramolecular hydrogen bonds of the amide groups resulting in a condensed globule. Consequently, the polymer becomes poorly soluble and the average diameter is shifted to higher values (see Figure 4.2). The LCST was chosen as the midpoint temperature of the aggregation step and was determined at a temperature of $31^{\circ} \mathrm{C}$.

A

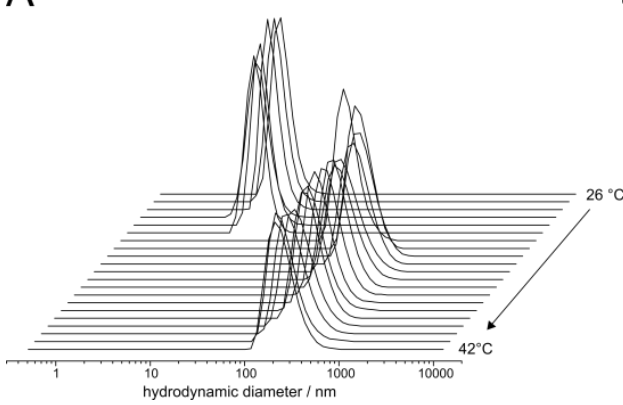

B

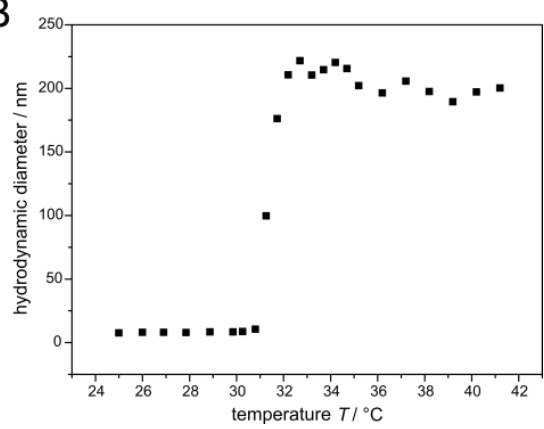

Figure 4.2. Number-averaged size distributions of the hydrodynamic diameter (A) and average hydrodynamic diameter in dependence of the temperature (B) of the free polymer in water.

Water contact angle measurements of the mesoporous silica thin films were performed in order to verify the successful functionalization (see Figure 4.3). Due to the hydrophobic nature of the RAFT agent, the water contact angle of the mesoporous films increased from $46^{\circ}$ to $60^{\circ}$ after derivatization (Figure 4.3). Afterwards, SI-RAFT polymerization was performed. This process led to the surface modification of the mesoporous film with covalently anchored PNIPAM brushes and a slight increase in the water contact angle from $60^{\circ}$ to $66^{\circ}$ which is in a good agreement with literature-known values.[262] 




Figure 4.3. Images of the water contact angle measurements of mesoporous silica thin films (MTF), modified with the BTP ${ }^{3} \mathrm{~T}$ RAFT agent (RAFT-MTF) and after SI-RAFT polymerization with NIPAM (PNIPAM-MTF).

Furthermore, water contact angle measurements below and above the LCST of PNIPAM were performed. Figure 4.4 shows the respective images. The water contact angle increases from $66^{\circ}$ to $72^{\circ}$ by raising the temperature above the LCST. By increasing the temperature the intermolecular hydrogen bonds are replaced by intramolecular bonds. Therefore, the collapsed polymer chains exhibit more hydrophobicity than below the LCST. The results are in good agreement with literature values.[240]

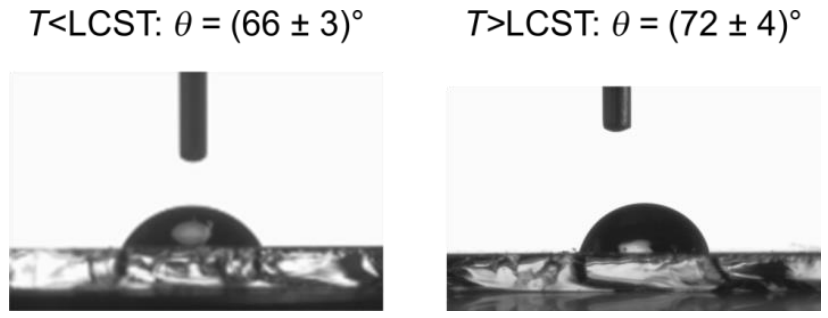

Figure 4.4. Images of the water contact angle measurements of mesoporous silica thin films after SI-RAFT polymerization with NIPAM (PNIPAM-MTF) below and above the LCST.

Additionally, the film was examined by environmental ellipsometric porosimetry (EEP) measurements. This method measures the changes in refractive index and thickness induced by the adsorption and condensation of water (or a gas) in the pores of the samples. Thus, adsorption-desorption isotherms are available and the pore-size distribution can be determined.[263] Figure 4.5a shows the adsorption-desorption-isotherms of the functionalized mesoporous silica thin film. The isotherms can be classified by IUPAC into type IV progression and a hysteresis H1 loop.[163] In general, the hysteresis loop $\mathrm{H} 1$ is characteristic of materials exhibiting a narrow range of uniform mesopores. Furthermore, the steep and narrow loop is 
attributed to a delay of condensation on the adsorption branch.[185] The measurements showed that the RAFT anchor groups do not block the pore. Moreover, after polymer growth the pore volume decreased slightly ( $\sim 5 \%)$. Nevertheless, a high pore volume remains, supporting the hypothesis of superficial brush growth. The hysteresis behavior changes with polymer presence due to a capping effect enlarging the hysteresis gap. A thin shoulder appears due to neck shrinkage in the mesoporous surface. From these measurements, the polymer layer was estimated to be $9 \mathrm{~nm}$ in thickness. In addition, the effect of the modification with RAFT and SIRAFT polymerization on the pore and neck sizes were extracted from the adsorption and desorption curves.[263,264] The measurements revealed only slight changes in pore and neck sizes indicating that polymer brushes did not extend far into the mesopores (Figure 4.5b).
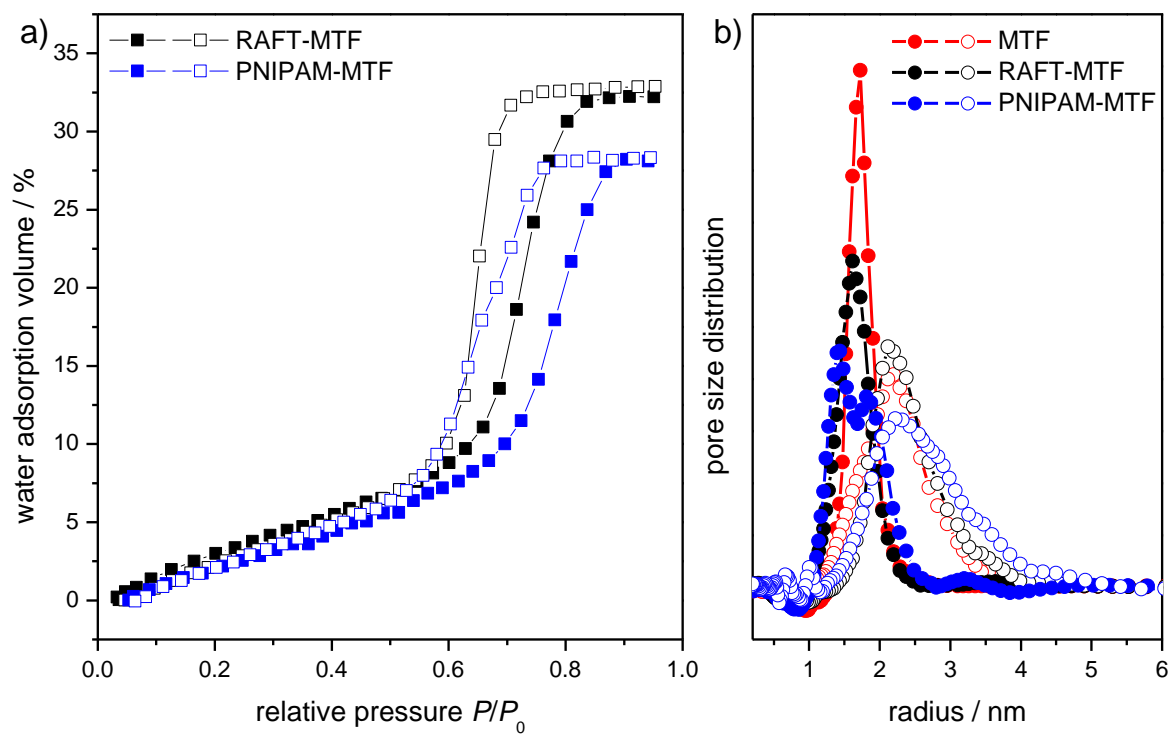

Figure 4.5. Adsorption-desorption isotherms (a) obtained using EEP of the functionalized mesoporous silica thin films (MTF) with RAFT agent (RAFT-MTF) and after subsequent SI-RAFT polymerization (PNIPAM-MTF). Filled symbols display the adsorption and open symbols display the desorption. Pore size distribution (b) of unmodified mesoporous silica films (MTF), mesoporous films modified with the RAFT agent (RAFT-MTF), and PNIPAM-modified mesoporous silica films (PNIPAM-MTF), as determined by EEP. Filled circles display the neck size and open circles display the pore.

However, XPS measurements were conducted to prove the surface- 
tethered PNIPAM brushes. A peak at around $399.7 \mathrm{eV}$ appeared which is in the region of the typical binding energy of nitrogen $(\mathrm{N} 1 \mathrm{~s})$. This result indicated the presence of the amide groups of PNIPAM, on the mesoporous surfaces after the polymerization (Figure 4.6a).[265] Furthermore, the XPS spectrum of the sample in the sulfur ( $2 \mathrm{p}$ ) region revealed the presence of the sulfur-containing RAFT agent on the mesoporous film (Figure 4.6b). Complementary, AFM imaging revealed the presence of a homogenously distributed nodular-like film grown on the mesoporous substrate while no patches or uncoated regions were found (Figure 4.7). From these results it can be inferred that the PNIPAM layer was grown atop the mesoporous film.
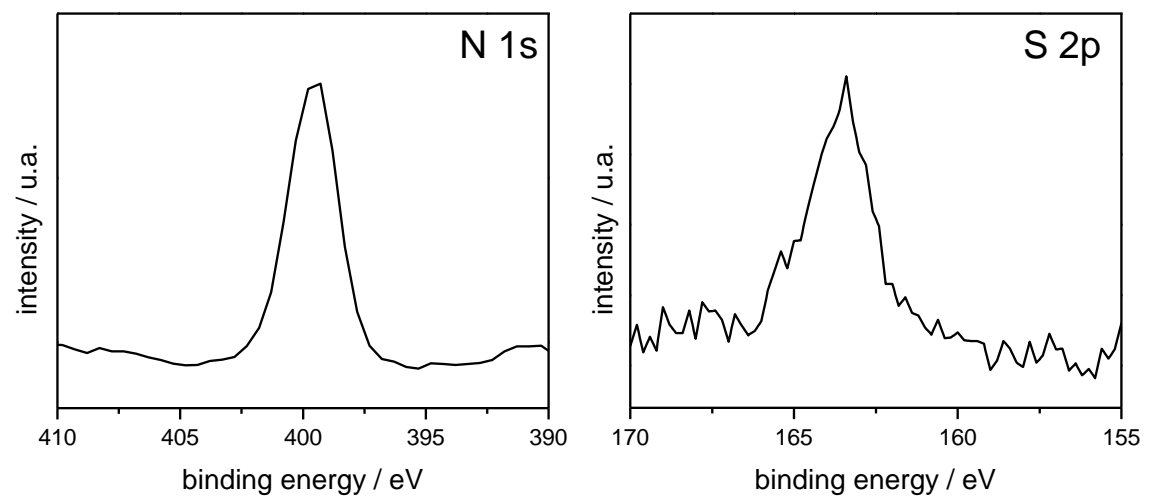

Figure 4.6. $\mathrm{N}$ 1s (left) and $\mathrm{S} 2 \mathrm{p}$ (right) $\mathrm{XP}$ spectra corresponding to the mesoporous silica film after PNIPAM polymerization.

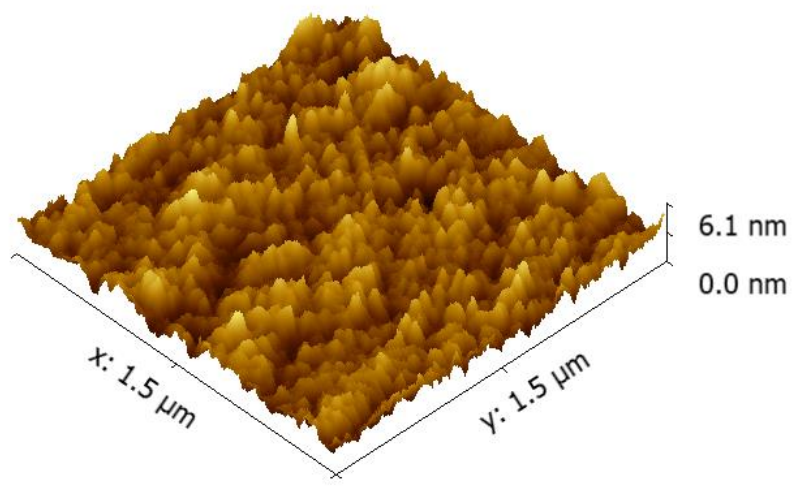

Figure 4.7. AFM three-dimensional topography image of the PNIPAM-modified mesoporous silica thin film (PeakForce Tapping ${ }^{\mathrm{TM}}$ mode, maximum $\mathrm{z}$-scale: $6 \mathrm{~nm}$; scan size $1.5 \times 1.5 \mu \mathrm{m})$. 
It can be hypothesized that the modification of mesoporous silica films with thermosensitive brushes is selective to the outer part of the film. It originates from the steric hindrance of the chains diffusing to the surfaceanchored Z-groups that precludes PNIPAM chains from entering into the mesopores. In other words, the Z-group approach can be considered as a grafting-to strategy as it relies on the diffusion of preformed polymer chains to the mesoporous surface. ${ }^{266]}$ This fact ultimately leads to the selective tailoring of the outer chemistry of the hybrid mesostructured assembly (see Scheme 4.4). In previous works, the combination of cationic monomers and silanolate surfaces led to partial or complete pore filling through controlled monomer concentration.[267,268] The use of a monomer containing a phosphoric acid group which was partially hydrolyzed led instead to polymerization within the pores and on top of the film surface. [210]
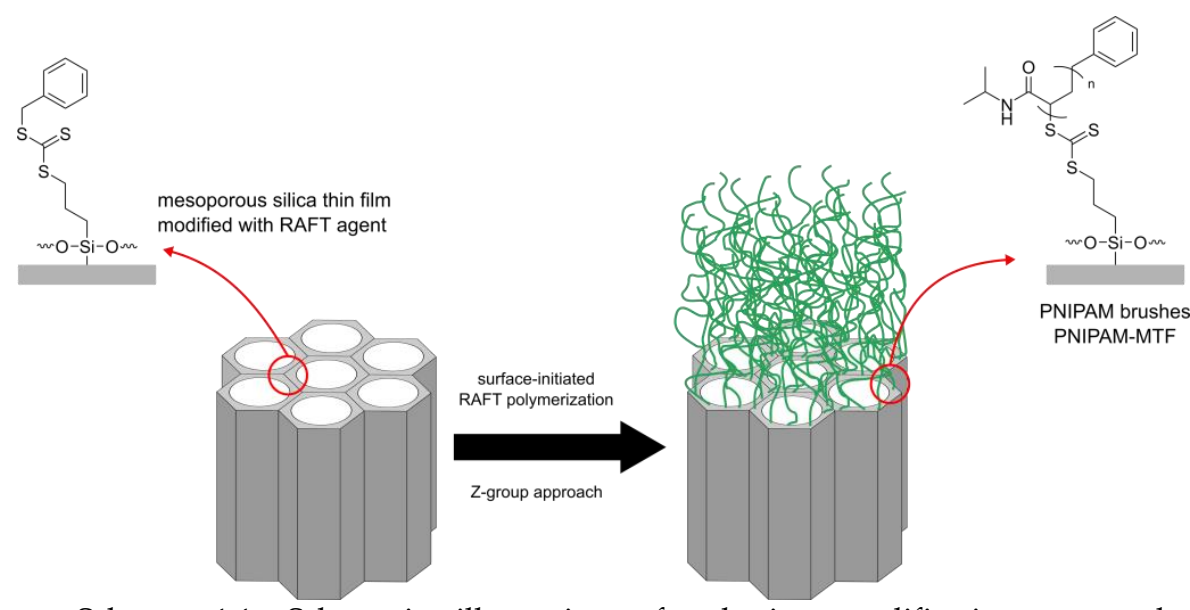

Scheme 4.4. Schematic illustration of selective modification upon the mesoporous silica thin film with RAFT agent and subsequently SI-RAFT polymerization.

\subsection{Cation-Selective Behavior of the Thermosensitive Mesoporous Silica Thin Film}

Once the formation of PNIPAM-capped mesoporous films was confirmed, studies of the thermo-dependent permselective properties of the mesostructured hybrid interface were conducted. The transport properties were characterized using redox probes that diffuse across the mesoporous 
film deposited on a conductive ITO substrate. Figure 4.8a displays the cyclic voltammograms of PNIPAM-capped mesoporous silica films on ITO (indium tin oxide) in the presence of $1 \mathrm{mM} \mathrm{Ru}\left(\mathrm{NH}_{3}\right)_{6}{ }^{3+}$ and $\mathrm{Fe}(\mathrm{CN})_{6}{ }^{3-}$ at $20^{\circ} \mathrm{C}$, respectively. A strong inhibition of the electrochemical signal of $\mathrm{Ru}\left(\mathrm{NH}_{3}\right)_{6}{ }^{3+}$ ions is observed, whereas no signal corresponding to $\mathrm{Fe}(\mathrm{CN})_{6}{ }^{3-}$ ions is detected. As suggested by Lopez and co-workers, below the LCST the swollen PNIPAM chains can act as an effective steric barrier and block the transport through the pore openings, which is caused by a strong decrease in the electrochemical signals of the redox probes. ${ }^{[240]}$ The working temperature was set to a point above the LCST and the transport of both redox probes was characterized (see Figure $4.8 \mathrm{~b}$ ). Due to heat loss in the experimental setup and to warranty temperature above the LCST it was set to $50{ }^{\circ} \mathrm{C}$. In contrast to results at $20^{\circ} \mathrm{C}$, upon increasing temperature above the LCST, the electrochemical response of $\mathrm{Ru}\left(\mathrm{NH}_{3}\right)_{6}{ }^{3+}$ strongly increased, leading to a well-defined voltammetric signal, whereas the signal corresponding to $\mathrm{Fe}(\mathrm{CN})_{6}{ }^{3-}$ remained fully inhibited. This temperatureinduced behavior is characterized as cation-selective ON state. According to the mechanism demonstrated by Lopez and collaborators, the collapsed PNIPAM chains unblock the pores above the LCST, thus allow the passage or diffusion of species from the solution into the mesopores. 


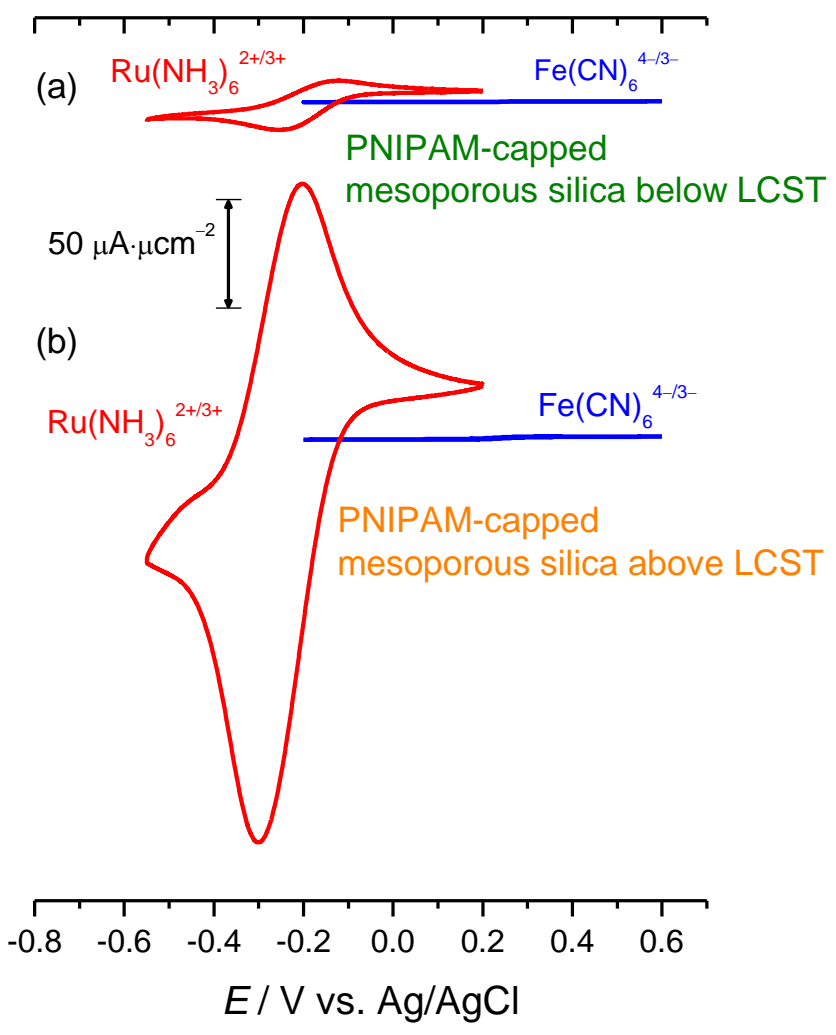

Figure 4.8. Cyclic voltammograms corresponding to: (a) PNIPAM-modified mesoporous silica thin film at $20^{\circ} \mathrm{C}$ (below LCST), (b) PNIPAM-modified mesoporous silica thin film at $50^{\circ} \mathrm{C}$ (above LCST), in the presence of $1 \mathrm{mM}$ $\mathrm{Ru}\left(\mathrm{NH}_{3}\right)_{6^{3+}}$ (red trace) and $1 \mathrm{mM} \mathrm{Fe}(\mathrm{CN})_{6}{ }^{3-}$ (blue trace), respectively. Scan rate: $100 \mathrm{mV} \cdot \mathrm{s}^{-1}$. Supporting electrolyte: $0.1 \mathrm{M} \mathrm{KCl}$.

The experimental data implies that the mesoporous film modified with collapsed PNIPAM chains on the pore outlets is acting as a very efficient permselective nanostructured barrier inhibiting the transport of anionic species. It is evident that the thermoactuation of the PNIPAM layer is transduced into drastic changes of the transport properties of the mesoporous film owing to the electrostatic nature of the inner environment of the mesopore matrix. The presence of surface-confined silanolate groups $\left(\mathrm{Si}-\mathrm{O}^{-}\right)$, is responsible for conferring permselective properties to the film at $\mathrm{pH}$ values above that of the silica surface $\mathrm{p} K_{\mathrm{a}} \approx 2 .{ }^{[267]}$ The exposed $\mathrm{SiO}^{-}$ groups act as an electrostatic barrier precluding the transport of anionic species. As a result, the uncapped pores operate as silanolate-gated, cation- 
selective mesochannels. They repel the transport of $\mathrm{Fe}(\mathrm{CN}) 6^{3-}$ but enable the free diffusion of $\mathrm{Ru}\left(\mathrm{NH}_{3}\right)_{6}{ }^{3+}$ ions across the inner environment of the mesoporous film. Figure 4.9 displays a histogram that reflects the relative changes in ionic transport properties under different temperature conditions for both probes. Considering, EEP confirmed that mesopores are not significantly occluded with polymer. It is plausible to ascribe the strong decrease in electrochemical signals to the presence of the steric barrier arising from the swollen PNIPAM brushes sitting atop the mesoporous film. Above the LCST, PNIPAM chains collapse onto the pore outlets concomitantly unblocking the mesopores (see Scheme 4.5).

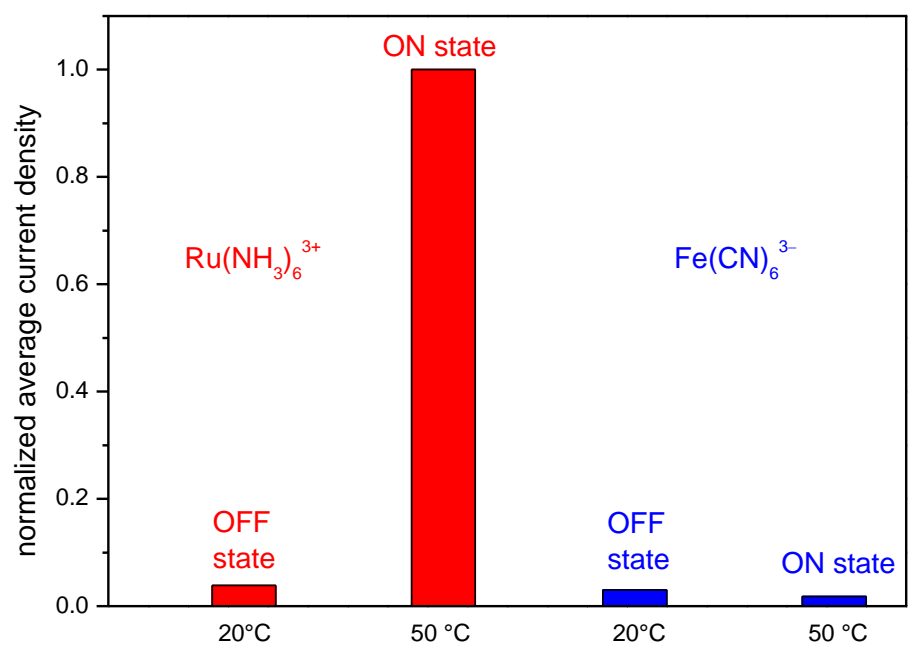

Figure 4.9. Histogram showing normalized variations in electrochemical current densities (ON and OFF states) arising from the influence of temperature on the molecular transport of $\mathrm{Ru}\left(\mathrm{NH}_{3}\right)_{6}{ }^{3+}$ and $\mathrm{Fe}(\mathrm{CN})_{6}{ }^{3-}$ redox probes through the PNIPAM-modified mesoporous silica films. 


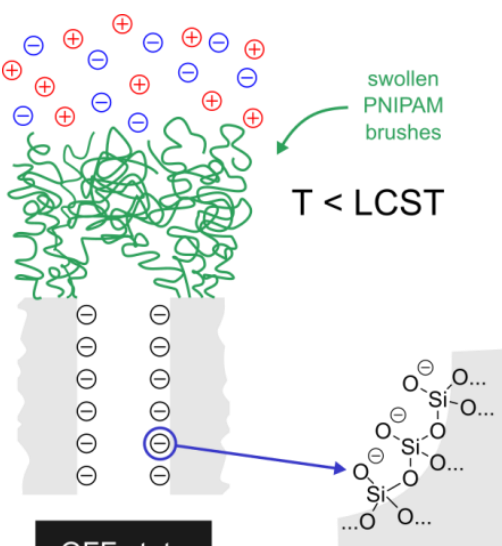

OFF state

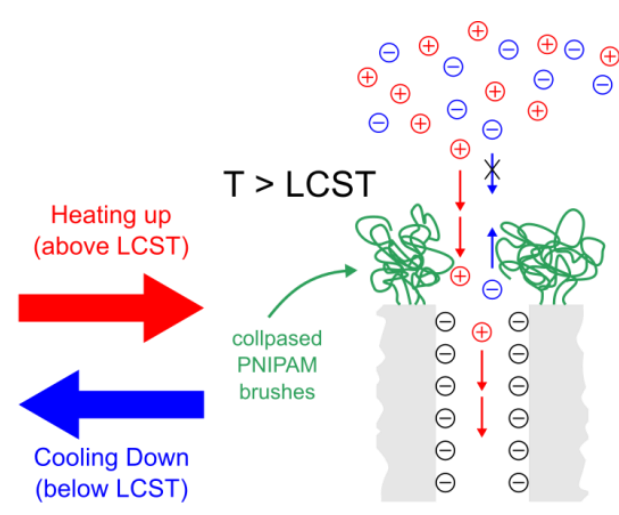

cation-selective ON state

Scheme 4.5. Schematic illustration of the ionic transport taking place in the hybrid polymer-inorganic interfacial assembly at temperatures below and above LCST.

Furthermore, the reversibility of this nanogating process was confirmed by cycling the temperature to a point above and below LCST. Figure 4.10 presents the reversible variation of the normalized electrochemical current of $\mathrm{Ru}\left(\mathrm{NH}_{3}\right)_{6}{ }^{3+}$ ions during consecutive temperature changes between $20^{\circ} \mathrm{C}$ and $50^{\circ} \mathrm{C}$. These measurements confirm the excellent reversible transport properties of the thermosensitive cation-selective mesochannels. 


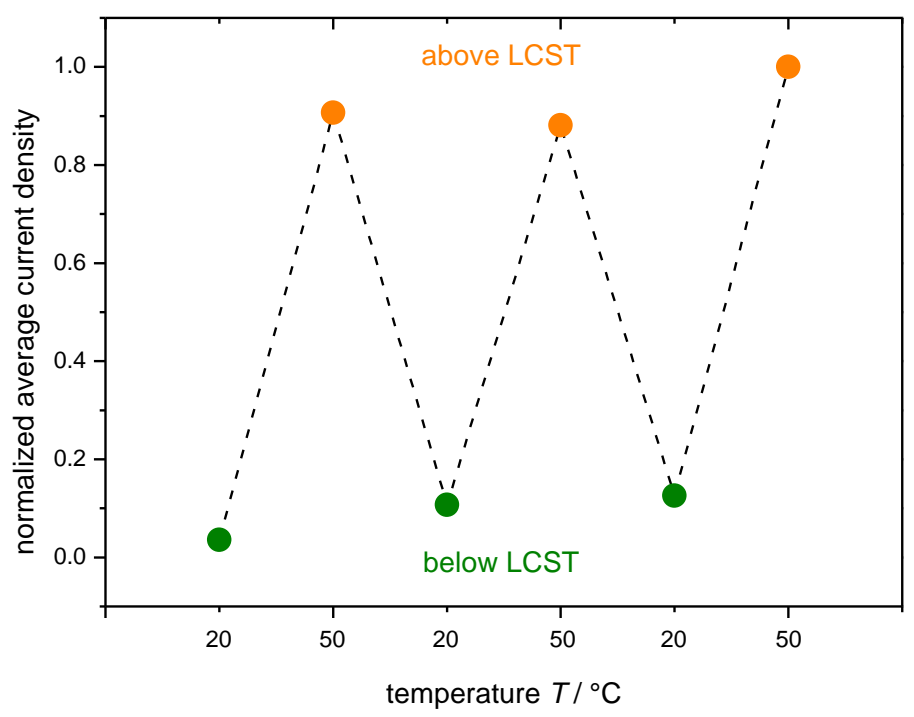

Figure 4.10. Reversible variation of the normalized electrochemical current of cationic $\mathrm{Ru}\left(\mathrm{NH}_{3}\right)_{6}{ }^{3+}$ redox probes diffusing through PNIPAM-modified mesoporous silica films upon alternating the solution temperature between $50^{\circ} \mathrm{C}$ (orange circles, ON state: above LCST) and $20^{\circ} \mathrm{C}$ (green, OFF state: below LCST). Supporting electrolyte: $0.1 \mathrm{M} \mathrm{KCl}$.

\subsection{Conclusion}

In this chapter, a strategy to synthesize a hybrid polymer-inorganic assembly of mesoporous silica thin films and tethered thermosensitive PNIPAM brushes was described. SI-RAFT polymerization using the Z-group approach was demonstrated to be a useful tool to selectively tether PNIPAM brushes onto the outer surface of the mesoporous silica thin films. Therefore, a RAFT agent exhibiting a silyl ether anchor group was bound to the mesoporous silica thin films and used in a polymerization with sacrificial RAFT agent in solution. The functionalization of the outer parts of the film was confirmed by EEP and XPS. Furthermore, the hybrid material was characterized by water contact angle measurements and AFM.

The electrochemical transport properties of the mesostructured hybrid interface were investigated revealing that thermo-dependent permselectivity was present. This is the first experimental report on thermo- 
activated permselectivity in mesoporous thin films using concerted functions inside and outside the mesoporous matrix. This synergy arises from the combination of the electrostatic characteristics of the silica scaffold and thermo-controlled steric effects introduced by the capping PNIPAM brush layer. In most examples of mesoporous silica used in delivery systems, nanoporous matrices serve purely as scaffolds to create nanoscopic channels. An important finding of this research is the creation of interfacial architectures capable of accomplishing specific functions through concerted or simultaneous action of different spatially-addressed pre-designed subunits. These results may open up new avenues for producing tailored interfacial architectures displaying spatially-addressed functions which is an emerging concept often referred to as nanoarchitectonics.[269-271] This may ultimately lead to heterosupramolecular nanosystems with novel molecular transport functions arising from concerted interactions between inorganic and macromolecular counterparts. 


\section{Closing Remarks and Future Perspectives}

The described microphase separation behavior of multiblock copolymers (chapter 2), their implementation as structure directing agents in the sol-gel process (chapter 3 ) and the approach to modify mesoporous films with responsive polymers (chapter 4) demonstrated the scope of tailor-made mesoporous structures by macromolecular building units on the nanoscale.

Multiblock copolymers $m$-(PS- $b$-PBA) and $m$-(PS- $b$-PNIPAM) which exhibit immiscible segments were synthesized via polyfunctional RAFT agents and systematically investigated on their microphase separation behavior via AFM. For the $m$-(PS- $b$-PBA) system, depending on the volume fraction of PBA segments cylindrical morphologies were obtained by thermal annealing. The different morphologies of the amphiphilic multiblock copolymers comprising PS and PNIPAM segments were investigated using selective solvents for annealing. It was demonstrated that the choice of solvent (mixture) can directly control the formation of a particular morphology due to different swelling of the film domains. Multiblock copolymers with a volume fraction $0.59<f_{\text {PNIPAM }}<0.77$ showed cylinders that were oriented perpendicular to the substrate using solvent mixtures of methanol-THF and methanol-toluene. Furthermore, the film thickness plays an important role in the formation of microdomains. In thicker films the formation of ordered structures is hampered compared to thinner films. Using selective solvent or solvent mixture, similar behavior could be observed for multiblock copolymers exhibiting three segments. Additionally, the inversion of morphology was achieved by combination of solvent annealing and thermal annealing. The temperature-dependence of the surface energy is more pronounced for the PS domains and thus transforms the structure into the inverted one. Future studies should examine the observed morphologies in SAXS experiments to verify the proposed structures. Moreover, different polymer systems can be adapted to the preparation by polyfunctional RAFT agents due to the versatility of the RAFT process. The prospect of being able to control the phase separation behavior by selective solvents opens up the possibility of new morphologies which are not accessible by thermal annealing. However, the 
level of swelling and degree of order in the solvent swollen films is unknown at this time, although these are issues to be resolved in future studies e.g., using ellipsometry and in situ grazing incidence small angle Xray scattering. Another important issue to resolve in future studies is to investigate the phase separation behavior of the cleavage products in detail. As an alternative to the cleavage reactions, $\mathrm{AB}$ block copolymers with matching composition and molar mass of the individual blocks could be prepared. This procedure would avoid impurities which might disturb the separation process.

Based on the ability to show phase separation in well-ordered morphologies, the amphiphilic multiblock copolymers were utilized as structure directing agents in order to form mesoporous silica films. A general synthetic strategy was developed using the solvent annealing to promote the mesostructured formation upon film deposition via evaporation-induced self-assembly process. Template removal by oxygen plasma resulted in large interconnected, unordered pores in a pore size range from $10-27 \mathrm{~nm}$. The formation of the mesoporous silica films is influenced by the preparation process and the nature of the multiblock copolymers. The most suitable sol aging time was determined to be 3 days. Longer reaction times lead to condensed silica species and thus no pore formation. The porosity is enhanced by increasing the concentration of the template in sol-gel solution due to reinforced interaction with the silica precursor. Furthermore, the pore size depends on the number of segments in the multiblock copolymers due to their arrangement in selective solution. Using multiblock copolymers exhibiting an average segment number below 4 resulted in large pores with a size greater than $16 \mathrm{~nm}$. In contrast, by increasing the average segment number in the amphiphilic template welldefined mesopores ranging from 10-15 nm were formed. Further studies may concentrate on the transport properties of those porous materials using redox probes. Furthermore, the large pore size opens up the pathway for artificial reaction channels for larger molecules than water. The design of porous material could be transferred to using non-silica oxides such as titanium or zirconium oxide. In addition, short sequences of sticky groups can be added to the multiblock copolymers which enhance the incorporation into the inorganic precursor. Future studies should aim to keep the functionalities of the multiblock copolymers instead of removing it in order to form the mesoporous framework.

Literature-established mesoporous silica thin films were tuned by postmodification methods. ${ }^{[155,259,260]}$ Polymer-mesoporous hybrid materials were synthesized using surface-initiated RAFT polymerization. Therefore, RAFT agents exhibiting silyl ether anchor groups were tethered to mesoporous silica films. Sequent polymerization from surface resulted in the selective 
functionalization of the outer sphere of the mesoporous film with PNIPAM brushes. The thermoresponsive behavior of the polymer brushes changed the hydrophilicity of the outer part of the mesoporous film. The modification allows to tune ionic permselectivity of charged redox probe molecules such as $\left[\mathrm{Fe}(\mathrm{CN})_{6}\right]^{4-/ 3-}$ and $\left[\mathrm{Ru}\left(\mathrm{NH}_{3}\right)_{6}\right]^{2+/ 3+}$ through the hybrid mesoporous material. Below the lower critical solution temperature, the polymer brushes are swollen and redox probes are excluded from the mesopores due to steric hindrance by the brushes. Thermoinduced gate opening leads to the permselective transport of cationic redox probes. The anionic redox probes are excluded by electrostatic exclusion of the mesopores. The synergy of stimuli-responsive behavior of polymers and electrostatic properties of the mesopores offers switchable cationic-selective mesochannels. Future studies may concentrate on the preparation of these interfacial architectures using different stimuli-responsive polymers. Even the combination of stimuli is possible such as thermo- and light-sensitive polymers. A suitable candidate as a light-sensitive component is the photochrome spiropyran molecule which can reversibly transform from a cyclic to open-ring form upon light irradiation. ${ }^{[272,273]}$ The open-ring merocyanine is formed by UV irradiation. Depending on the $\mathrm{pH}$ the merocyanine is either positively charged or a zwitterion. The transformation back to the closed spiropyran can be induced by heat or by irradiation with visible light. Aznar et al. showed the successful lightinduced mesoporous gating system using the switch-ability of the spiropyran combined with G1.5 PAMAM dendrimer. ${ }^{[272]}$ Combining these light-sensitive properties with those of thermosensitive PNIPAM might lead to novel molecular gating functions.

To conclude, mesoporous materials are tunable by direct tailoring of the macromolecular precursor and by post-modification with stimuliresponsive polymers. Furthermore, the presented results open up the pathway for future applications like sensors or catalysis. 


\section{Experimental Section}

\subsection{Chemicals}

$\mathrm{N}$-isopropylacrylamide (NIPAM, TCI-Chemicals, 97\%) and 2,2'-Azobis(methylpropionitrile) (AIBN, Akzo Nobel, 98\%) were recrystallized twice from toluene/hexane (3:1) and diethyl ether and dried in vacuum. Styrene and $n$-butyl acrylate were passed through a basic alumina column prior to use and were stored at $-18^{\circ} \mathrm{C}$. Silicon wafer substrates ([100]) were purchased from Plano and exhibited a size of $10 \times 10 \mathrm{~mm}$. The silicon substrates were plasma-cleaned prior to use. All other chemicals were purchased from Sigma Aldrich and used without further purification. Benzyl (3-(trimethoxysilyl)propyl)trithiocarbonate (BT3PT) was synthesized recording the literature and kindly provided by Dr. Julia Möhrke.[244,246]

\subsection{Analytical Methods}

\subsubsection{Atomic Force Microscopy}

Atomic force microscopy (AFM) measurements were performed on a Multimode AFM (Bruker) with a NanoScope V controller with ScanAsystAir HR cantilever (Bruker, nominal spring constant: $0.4 \mathrm{~N} \cdot \mathrm{m}^{-1}$, nominal resonance frequency: $70 \mathrm{kHz}$ ) or TAP525A cantilever (Bruker, nominal spring constant: $200 \mathrm{~N} \cdot \mathrm{m}^{-1}$, nominal resonance frequency: $525 \mathrm{kHz}$, nominal radius $8 \mathrm{~nm}$ ) at room temperature. For measuring either the PeakForce Tapping ${ }^{\mathrm{TM}}$ mode or the PeakForce-Quantitative Nanomechanical Mapping $^{\mathrm{TM}}$ (PeakForce-QNM ${ }^{\mathrm{TM}}$ ) mode was used. All images were taken with a scan rate of $3.92 \mathrm{~Hz}$ (PeakForce Tapping ${ }^{\mathrm{TM}}$ ) or $0.97 \mathrm{~Hz}$ (PeakForce$\mathrm{QNM}^{\mathrm{TM}}$ ) and a resolution of $512 \times 512$ samples per line. For the analysis of the data the program Gwyddion (Departement of Nanometrology, Czech Metrology Institute) was used. 


\subsubsection{Size-Exclusion-Chromatography}

Size-Exclusion-Chromatography (SEC) with N,N-dimethylacetamide (DMAc) containing $0.1 \mathrm{wt} \%$ of lithium bromide as eluent was performed using an Agilent 1260 Infinity system. It was composed of an autosampler, an isocratic solvent pump, a PSS GRAM (polyester copolymer network) precolumn $(8 \times 50 \mathrm{~mm})$, three PSS GRAM separation columns $(8 \times 300 \mathrm{~mm}$, nominal particle size $=10^{-5} \mathrm{~m}$; pore sizes $=30,10^{3}$, and $10^{3} \AA$ ) maintained at $45^{\circ} \mathrm{C}$ in a column compartment, an $80 \mathrm{~Hz}$ UV detector (set to a wavelength of $310 \mathrm{~nm}$ for the RAFT polymers), and a refractive index (RI) detector. The flow rate of the mobile phase was $8 \times 10^{-4} \mathrm{~L} \cdot \mathrm{min}^{-1}$. The whole setup was calibrated with 12 PSS poly(methyl methacrylate) standards $\left(M_{n}=0.8-1820 \mathrm{~kg} \cdot \mathrm{mol}^{-1}\right)$ of low dispersity with toluene as internal standard. Molar masses of PNIPAM were determined by using the MarkHouwink parameters of the PMMA/THF-system (Mark-Houwinkcoefficient $K=0.01298 \mathrm{~mL} \cdot \mathrm{g}^{-1}, \alpha=0.688$, universal calibration). All samples were filtered through a $450 \mathrm{~nm}$ PTFE syringe filter prior to injection. The concentration of the polymer samples were $3 \mathrm{~g} \cdot \mathrm{L}^{-1}$.

SEC characterizations with THF as eluent were performed using Agilent1260 Infinity system. It consisted of an autosampler, an isocratic HPLC pump, a PSS SDV precolumn $(8 \times 50 \mathrm{~mm})$, three PSS SDV separation columns $\left(8 \times 300 \mathrm{~mm}\right.$, particle size $=10 \mu \mathrm{m}$, pore sizes $=10^{6} \AA, 10^{5} \AA$, and $10^{3} \AA$ ) , maintained at $35^{\circ} \mathrm{C}$ in a column compartment, an $80 \mathrm{~Hz}$ UV detector (set to a wavelength of $310 \mathrm{~nm}$ ) and an RI detector. The flow rate of the mobile phase was $1 \mathrm{~mL} \cdot \mathrm{min}^{-1}$. The SEC setup was calibrated with lowdispersity PSS standards $\left(M_{p}=0.5-2520 \mathrm{~kg} \cdot \mathrm{mol}^{-1}\right)$ with toluene as internal standard. Molar masses of PBA were determined according to the principle of universal calibration using the Mark-Houwink parameters for the linear polymer $\left(K=1.22 \cdot 10^{-4} \mathrm{dL} \cdot \mathrm{g}^{-1}, a=0.700\right)$. The concentration of the samples was $3 \mathrm{~g} \cdot \mathrm{L}^{-1}$.

\subsubsection{Mass Spectroscopy}

Electron spray ionization (ESI) spectra were measured with a Triplet quadrupole instrument TSQ 7000 or an ion trap mass spectrometer LCQ of the company Finnigan. The measurement of ESI high resolution mass spectrometry (HRMS) spectra were performed on a 7 Tesla Fourier transform ion cyclotron resonance (FTICR) mass spectrometer Bruker which is equipped with an Apollo source of Bruker and a syringe pump 74900 Series of the company Cole-dPalmer. 


\subsubsection{Nuclear Magnetic Resonance Spectroscopy}

Nuclear magnetic resonance (NMR) spectra ( ${ }^{1} \mathrm{H}-\mathrm{NMR}$ and $\left.{ }^{13} \mathrm{C}-\mathrm{NMR}\right)$ were measured with a Varian Unity 300 instrument at room temperature. The samples were dissolved either in deuterated chloroform, dichloromethane or acetone. For the spectra residual solvent proton signals were used as internal standard.

\subsubsection{Scanning Electron Microscopy}

Scanning electron microscopy (SEM) images were obtained either with a ZEISS LEO GEMINI field emission electron microscope (CMA, Facultad de Ciencias Exactas y Naturales, UBA, Argentina) by Sebastián Alberti or with a Type FEI Nova NanoSEM 650 scanning electron microscope at an operating voltage of $2 \mathrm{kV}$. For the analysis of the data the program ImageJ was used.

\subsubsection{Thermogravimetric Analysis}

Thermogravimetric analyses (TGA) were measured with a Netzsch TG $209 \mathrm{~F} 3$ Tarsus from $25^{\circ} \mathrm{C}$ up to $1000{ }^{\circ} \mathrm{C}$ with a heating rate of $10^{\circ} \mathrm{C} \cdot \mathrm{min}^{-1}$ under a nitrogen flow rate of $10 \mathrm{~mL} \cdot \mathrm{min}^{-1}$.

\subsubsection{Water Contact Angle}

Contact angle (CA) was measured with an OCA 15EC (OCA Measuring Instruments) via the TBO Video-based contact angle measuring system by Dataphysics. A water drop $(2 \mu \mathrm{L})$ was placed at five different spots on the surfaces. The presented results are an average of the five measurements.

\subsubsection{Ellipsometry}

The thicknesses of the thin polymer films were measured by spectroscopic ellipsometer Nanofilm EP4 (Accurion). Measurements were performed over a wavelength range of 360 to $800 \mathrm{~nm}$ with a laser stabilized xenon arc lamp at an angle of incidence of $50^{\circ}$. The film thicknesses of $m$-(PS- $b$-PBA) and $m$-(PS- $b$-PNIPAM) were determined by fitting the optical properties of the polymer layer with the Cauchy model. The mesoporous silica thin films were fitted with the EM-Brüggeman model.

Film thickness and refractive index of the mesoporous thin films in the 
200-950 nm regions were obtained using a SOPRA GES5A spectroscopic ellipsometer. Measurement of the ellipsometric parameters $\Psi$ (Theta) and $\Delta$ (Delta) were carried out under dry nitrogen flux in order to avoid water condensation within the mesopores. The film refractive index was satisfactorily adjusted according to a one-layer model for both RAFT agent functionalized and the polymer-modified mesoporous films. A Brüggemann effective medium approximation (BEMA) was used to calculate the pore volume fraction $\left(V_{\text {pore }} / \%\right)$, considering two phases made up of silica $\left(n_{633}=1.455\right)$ and void pores. The polymer volume fraction in the material $V_{\text {PNIPAM }} / \%$ was obtained by analyzing the optical data with a three component BEMA using $n_{633}=1.455$ for the silica framework, $n_{633}=1.50$ for PNIPAM and void pores. Water adsorption-desorption curves (at $298 \mathrm{~K}$ ) were measured by ellipsometric porosimetry (EP, SOPRA GES5A). Film thickness and refractive index values were obtained from measuring the ellipsometric parameters $\Psi$ (Theta) and $\Delta$ (Delta) under nitrogen flux containing controlled water vapor quantities; $P / P_{0}$ was varied from 0 to 1 ( $P_{0}$ being the saturation water vapor at $\left.298 \mathrm{~K}\right)$. Film pore volume and pore size distribution at each $P / P_{0}$ were obtained by modelling the refractive index obtained according to a three-medium BEMA (see above) corresponding to a one layer model for the mesoporous films.

\subsubsection{Cyclic Voltammetry}

Cyclic voltammetry (CV) was performed using a Gamry potentiostat with an $\mathrm{Ag} / \mathrm{AgCl}$ reference electrode. The probe solutions were prepared with a concentration of $1 \mathrm{mM}$ in $100 \mathrm{mM} \mathrm{KCl}$ (aqueous solution) as supporting electrolyte resulting in a $\mathrm{pH}=5-6$ solution. Quantitative variations in permselectivity were studied by observing the changes of voltammetric peak currents associated to cationic $\left[\mathrm{Ru}\left(\mathrm{NH}_{3}\right)_{6}\right]^{2+/ 3+}$ and anionic $\left[\mathrm{Fe}(\mathrm{CN})_{6}\right]^{4^{-} / 3^{-}}$redox probes, diffusing across the mesoporous film.

\subsubsection{Dynamic Light Scattering}

Dynamic light scattering (DLS) measurements were conducted on a Zetasizer Nano S (Malvern) with a He-Ne laser $(\lambda=633 \mathrm{~nm})$ by using $10 \mathrm{~mm}$ quartz cuvettes. Intensity distributions were recorded with 12 runs with duration of $20 \mathrm{~s}$ per measurements. All measurements were recorded under a detection angle of $173^{\circ}$. 


\subsubsection{Differential Scanning Calorimetry}

Differential scanning calorimetry (DSC) was obtained with a DScalorimeter Mettler Toledo DSC820 equipped with cryostat (Lauda UltraKryomat ${ }^{\circledR}$ RUK 90). Measurements were performed with a heating rate of $10^{\circ} \mathrm{C} \cdot \mathrm{min}^{-1}$ in a temperature range from $30^{\circ} \mathrm{C}$ up to $180^{\circ} \mathrm{C}$ for $m$-(PS- $b$ PNIPAM) with a constant nitrogen flow. The heat flow of the polymer sample was measured in an aluminum pan against a reference pan. The samples were first heated over the temperature range and cooled again. The displayed curves correspond to the second heating process. Further measurements were performed with a DS-calorimeter Shimadzu DSC-60 equipped with a TA-60WS Thermal Analysis System. For $m$-(PS- $b$-PBA) the temperature range was set from $-120^{\circ} \mathrm{C}$ to $180{ }^{\circ} \mathrm{C}$ with a heating rate of $10^{\circ} \mathrm{C} \cdot \mathrm{min}^{-1}$.

\subsubsection{X-ray Photoelectron Spectroscopy}

X-ray photoelectron spectroscopy (XPS) was performed using a SPECS SAGE HR 100 system spectrometer. A Mg K $(1253.6 \mathrm{eV})$ X-ray source was employed operating at $12.5 \mathrm{kV}$ and $10 \mathrm{~mA}$. Survey spectra were obtained with pass energy of $15 \mathrm{eV}$ was employed for detailed spectra of S2p region. The take-off angle was $90^{\circ}$ and operating pressure was $8 \cdot 10^{-8}$ mbar.

\subsubsection{Small-Angle X-ray Scattering}

Synchrotron-based small-angle X-ray scattering (SAXS) characterization was performed at the D10A-XRD2 beamline of Laboratório Nacional de Lus Síncrotron (LNLS-Brazil).

\subsubsection{Nitrogen Adsorption-Desorption Isotherms}

Nitrogen absorption-desorption experiments were characterized using a Gemini V - Surface Area and Pore Size Analyzer Micromeritic. Adrian Ley kindly performed the measurements. The saturated pressure was set to $756.9 \mathrm{mmHg}$ and the equilibration time to $5 \mathrm{~s}$. 


\subsection{Syntheses}

\subsubsection{Syntheses of RAFT Agents}

\subsubsection{Synthesis of 2,2'-(Thiocarbonylbis(sulfanediyl))- dipropanoic acid (MATC) ${ }^{[274,275]}$}<smiles>CC(SC(=S)SC(C)C(=O)O)C(=O)O</smiles>

To a solution of $\mathrm{KOH}(6.60 \mathrm{~g}, 0.12 \mathrm{~mol}, 1.0$ eq.) in water $(75 \mathrm{~mL})$ carbon disulfide $(6.35 \mathrm{~mL}, 0.11 \mathrm{~mol}, 0.9$ eq.) was added and the reaction mixture was stirred for $1 \mathrm{~h}$ at room temperature. Afterwards 2-bromopropionic acid (4.73 g, $0.05 \mathrm{~mol}, 0.4 \mathrm{eq})$ was added dropwise and mixture was stirred for further $72 \mathrm{~h}$. Then the solution was washed with methylene chloride $(5 \times 25 \mathrm{~mL})$ to remove unreacted carbon disulfide. The aqueous layer was acidified to $\mathrm{pH}=5$ with concentrated hydrochloric acid and then extracted with methylene chloride until aqueous layer was colorless. The organic layer was dried over magnesium sulfate, filtered and dried in vacuo. A yellow solid was obtained. The crude product was recrystallized from toluene/ethyl acetate $1: 1(\mathrm{v}: \mathrm{v})$ to yield the yellow solid $(6.10 \mathrm{~g}, 0.024 \mathrm{~mol}$, $20 \%)$.

${ }^{1}$ H-NMR $(300 \mathrm{MHz}, \mathrm{MeOD}): \delta(\mathrm{ppm})=13.24(\mathrm{~s}, 2 \mathrm{H}, 1-\mathrm{H}), 4.90-4.72(\mathrm{q}$, $J=8.00,6.50 \mathrm{~Hz}, 2 \mathrm{H}, 3-\mathrm{H}), 1.68-1.42(\mathrm{~d}, J=6.50 \mathrm{~Hz}, 6 \mathrm{H}, 2-\mathrm{H})$.

${ }^{13} \mathrm{C}-\mathrm{NMR}$ (75 MHz in MeOD): $\delta(\mathrm{ppm})=221.95$ (C-4), 173.95 (C-1), 49.71 (C-3), $17.30(\mathrm{C}-2)$

\subsubsection{Synthesis of the Br-TEG-Br Linker}<smiles>CC(Br)C(=O)OCCOCCOCCOCCOC(=O)C(Br)I</smiles>

Tetraethylene glycol (TEG, $4.36 \mathrm{~g}, 28.0 \mathrm{mmol}, 1.0 \mathrm{eq}$.$) and$ trimethylamine $(16.0 \mathrm{~mL}, 191 \mathrm{mmol}, 6.8$ eq.) were dissolved in THF $(150 \mathrm{~mL})$. The mixture was cooled to $0{ }^{\circ} \mathrm{C}$ and a solution of 2-bromopropionyl bromide (21.2 mL, $191 \mathrm{mmol}, 6.8$ eq.) in THF (40 mL) was added dropwise. The reaction mixture was allowed to warm up to 
room temperature and was stirred for further $2 \mathrm{~d}$. Afterwards, demineralized water was added $(80 \mathrm{~mL})$ and the aqueous phase was extracted with diethyl ether $(3 \times 80 \mathrm{~mL})$. The organic layers were combined and washed with a saturated sodium hydrogen carbonate solution, demineralized water $(3 \times 80 \mathrm{~mL})$ and dried over magnesium sulfate. The solvent was removed by evaporation and the product was purified via column chromatography using hexane:ethyl acetate $1: 1(\mathrm{v}: \mathrm{v})$ to yield a brownish oil (9.06 g, $19.5 \mathrm{mmol}, 70 \%)$.

${ }^{1} \mathrm{H}-\mathrm{NMR} \quad\left(300 \mathrm{MHz}, \mathrm{CDCl}_{3}\right): \delta(\mathrm{ppm})=4.40(\mathrm{q}, J=7.0 \mathrm{~Hz}, 2 \mathrm{H}, 2-\mathrm{H})$, 4.36-4.25 (m, 4H, 3-H), 3.77-3.69 (m, 4H, 4-H), $3.66(\mathrm{~s}, 8 \mathrm{H}, 5,6-\mathrm{H}), 1.82(\mathrm{~d}$, $J=7.0 \mathrm{~Hz}, 6 \mathrm{H}, 1-\mathrm{H})$.

${ }^{13} \mathrm{C}-\mathrm{NMR}\left(75 \mathrm{MHz}\right.$ in $\left.\mathrm{CDCl}_{3}\right): \delta(\mathrm{ppm})=170.23(\mathrm{C}=\mathrm{O}), 70.70(\mathrm{C}-6), 70.65(\mathrm{C}-$ 5), 68.78 (C-4), 65.02 (C-3), 39.90 (C-2), 21.63 (C-1).

MS (ESI) $m / z=487.0[\mathrm{M}+\mathrm{Na}+\mathrm{H}]^{+}$

ESI-HRMS $\quad \mathrm{C}_{14} \mathrm{H}_{24} \mathrm{Br}_{2} \mathrm{O}_{7}$ calc.: 486.9761

experiment: $486.9753[\mathrm{M}+3 \mathrm{H}]^{+}$.

\subsubsection{Synthesis of polyRAFT $A$ and $\mathbf{B}^{[15,36,37,276]}$}<smiles>C=C(NC)C(=O)OCCOC(=O)C(C)SC(=S)SC(=C)C(=O)OCCOC(=O)C(=C)SC(=S)SC(C)C(=O)OCCOCCOC(=O)C(C)Br</smiles>

The synthesis of polyRAFT A and $\mathbf{B}$ were accomplished by following the procedure reported by Ebeling et al. with minor changes. ${ }^{[15]}$ The dried anion exchange resin Ambersep ${ }^{\circledR} 900 \mathrm{OH}$ (1.03 g, $3.64 \mathrm{mmol}, 1.0$ eq.) was suspended in carbon disulfide $(5.15 \mathrm{~mL}, 3.64 \mathrm{mmol}, 1.0$ eq.). The resin beads turned dark red immediately. Br-TEG-Br (1.59 g, $1.82 \mathrm{mmol}, 0.50$ eq.) was added. The suspension was heated up and stirred for 5 days under reflux. Afterwards the reaction mixture was cooled to room temperature and the resin was filtered off. The beads were washed first with carbon disulfide $(300 \mathrm{~mL})$ and with THF $(300 \mathrm{~mL})$. The fractions were concentrated under reduced pressure, washed with water and methanol, dried in vacuo.

${ }^{1}$ H-NMR $\left(300 \mathrm{MHz}, \mathrm{CDCl}_{3}\right): \delta(\mathrm{ppm})=4.79\left(\mathrm{q}, J=7.1 \mathrm{~Hz}, \mathrm{H}_{\mathrm{b}}\right), 4.47-4.12(\mathrm{~m}$, $\left.\mathrm{H}_{\mathrm{c}}, \mathrm{H}_{\mathrm{g}}\right), 3.81-3.57\left(\mathrm{~m}, \mathrm{H}_{\mathrm{d}}, \mathrm{H}_{\mathrm{e}}, \mathrm{H}_{\mathrm{f}}\right), 1.83\left(\mathrm{~d}, J=7.1 \mathrm{~Hz}, \mathrm{H}_{\mathrm{a}}\right), 1.66-1.56\left(\mathrm{~m}, \mathrm{H}_{\mathrm{h}}\right)$. 


\subsubsection{Synthesis of polyRAFT $\mathrm{C}^{[61]}$}<smiles>CNC(C)(C)OCCOCCOCC(I)COCCOC(=O)C(C)SC(=S)SC(C)C(C)=O</smiles>

The synthesis of polyRAFT C was accomplished by following the procedure reported by Liu et al. with minor changes. MATC $(2.79 \mathrm{~g}$, $10.9 \mathrm{mmol}, 1.0$ eq.), TEG (2.13 g, $10.9 \mathrm{mmol}, 1.0$ eq.) and $p$-toluenesulfonic acid monohydrate $(0.42 \mathrm{~g}, 2.20 \mathrm{mmol}, 0.20 \mathrm{eq}$. $)$ were suspended in cyclohexane $(5.70 \mathrm{~mL})$. The reaction mixture was heated to $130{ }^{\circ} \mathrm{C}$ for $36 \mathrm{~h}$. Afterwards the mixture was cooled to room temperature and the obtained brown solid was dissolved in a small amount of dichloromethane and precipitated in an excess of cold methanol. The multifunctional RAFT agent was collected by centrifugation. The product was dried in vacuo and the molar mass of the compound was determined by SEC.

${ }^{1}$ H-NMR $\left(300 \mathrm{MHz}, \mathrm{DMSO}-\mathrm{d}_{6}\right): \delta(\mathrm{ppm})=4.73\left(\mathrm{q}, J=7.3 \mathrm{~Hz}, \mathrm{H}_{\mathrm{b}}\right), 4.20(\mathrm{~d}$, $J=4.3 \mathrm{~Hz} \mathrm{H}$ ), $3.60\left(\mathrm{t}, J=4.3 \mathrm{~Hz} \mathrm{H} \mathrm{d}_{\mathrm{d}}\right), 3.52\left(\mathrm{~d}, J=4.3 \mathrm{~Hz}, \mathrm{H}_{\mathrm{e}}, \mathrm{H}_{\mathrm{f}}\right), 1.54(\mathrm{~d}$, $\left.J=7.3 \mathrm{~Hz}, \mathrm{H}_{\mathrm{a}}\right)$.

\subsection{Synthesis of Mesoporous Silica Thin Films}

\subsubsection{Mesoporous Silica Thin Films with Block Copolymer}

Synthesis of mesoporous silica thin films was performed as described in previous works via processing an ethanolic solution containing the oxide precursor tetraethoxysilane (TEOS) in the presence of the template (F127 block copolymer, Aldrich, $\left.M_{\mathrm{n}}=13600 \mathrm{~g} \cdot \mathrm{mol}^{-1}\right)$.[277] The precursor solution was prepared using TEOS (20.83 g), ethanol (13.82 g), concentrated hydrochloric acid $(1.82 \mathrm{mg})$ and distilled water $(18 \mathrm{~g})$. The solution was heated up under reflux for $1 \mathrm{~h}$. A mixture of EtOH (17.05 g), concentrated $\mathrm{HCl}(2.89 \mathrm{mg})$, water $(1.62 \mathrm{~g})$ and F127 (0.69 g) was added to the precursor solution $(3.64 \mathrm{~g})$ and stirred at room temperature for $3 \mathrm{~d}$. This solution was used to prepare the mesoporous films by dip coating (withdrawing speed $3 \mathrm{~mm} \cdot \mathrm{s}^{-1}$ ) on glass and ITO substrates under $\sim 40 \%$ relative humidity conditions at ambient temperature. The organic template was removed by calcination at $350^{\circ} \mathrm{C}$ for $2 \mathrm{~h}$. 


\subsubsection{Mesoporous Silica Thin Films via Multiblock Copolymers}

TEOS (0.11 g), $\mathrm{HCl}(1 \mathrm{M}, 0.055 \mathrm{~g})$ and THF (3.0 g) were mixed and stirred for $24 \mathrm{~h}$ at room temperature. The respective polymer was dissolved in THF (1.0 g) and added to the previous TEOS-solution. The solution was then heated for $15 \mathrm{~min}$ at $70{ }^{\circ} \mathrm{C}$. Afterwards the solution was stirred further for $1 \mathrm{~d}(3$ or $7 \mathrm{~d})$. Afterwards the solution $(20 \mu \mathrm{L})$ was spin coated onto a plasma-cleaned silicon wafer $\left(3500 \mathrm{rpm}, 15 \mathrm{~s}, \mathrm{RH}<5 \%, \mathrm{~T}=23^{\circ} \mathrm{C}\right)$. Then the film was exposed to saturated MeOH-THF 2:1 (v:v) vapor for $2 \mathrm{~h}$ in a closed solvent annealing chamber. In order to quench the solvent annealing the chamber was evacuated to kinetically trap the system. Alternatively different structure giving methods were applied (see Table 6.1). The mesoporous film was recovered by calcined at either $450{ }^{\circ} \mathrm{C}$ for $1 \mathrm{~h}$ or in an $\mathrm{O}_{2}$ plasma oven (employing three times the plasma).

Table 6.1. Amount of multiblock copolymer (MBC), stirring time, structural giving workup (SVA : solvent annealing) and calcination process.

\begin{tabular}{ccccccc}
\hline sample & $\begin{array}{c}\text { MBC } \\
\text { template }\end{array}$ & $\begin{array}{c}\text { MBC } \\
\text { /g }\end{array}$ & $\begin{array}{c}\text { final } \\
\text { solution } \\
\text { stirring }\end{array}$ & $\begin{array}{c}\text { structural } \\
\text { promoting } \\
\text { workup }\end{array}$ & $\begin{array}{c}\text { template } \\
\text { removal }\end{array}$ & $\begin{array}{c}\text { porous } \\
\text { structure }\end{array}$ \\
\hline MP1 & X & 0.02 & $1 \mathrm{~d}$ & SVA & Plasma & Yes \\
MP2 & X & 0.02 & $1 \mathrm{~d}$ & SVA & $450{ }^{\circ} \mathrm{C}$ & Yes \\
MP3 & X & 0.02 & $1 \mathrm{~d}$ & None & Plasma & No \\
MP4 & X & 0.02 & $1 \mathrm{~d}$ & None & $450{ }^{\circ} \mathrm{C}$ & Yes \\
MP5 & IX & 0.02 & $1 \mathrm{~d}$ & SVA & Plasma & Yes \\
MP6 & IX & 0.02 & $1 \mathrm{~d}$ & SVA & $450{ }^{\circ} \mathrm{C}$ & Yes \\
MP7 & IX & 0.02 & $1 \mathrm{~d}$ & None & $450{ }^{\circ} \mathrm{C}$ & No \\
MP8 & V & 0.02 & $1 \mathrm{~d}$ & SVA & Plasma & Yes \\
MP9 & V & 0.02 & $1 \mathrm{~d}$ & SVA & $450{ }^{\circ} \mathrm{C}$ & No \\
MP10 & V & 0.02 & $1 \mathrm{~d}$ & None & Plasma & Incomplete \\
MP11 & V & 0.02 & $1 \mathrm{~d}$ & None & $450{ }^{\circ} \mathrm{C}$ & Yes \\
MP12 & IX & 0.02 & $3 \mathrm{~d}$ & SVA & Plasma & Yes \\
MP13 & V & 0.02 & $3 \mathrm{~d}$ & SVA & Plasma & Yes \\
MP14 & IX & 0.02 & $7 \mathrm{~d}$ & SVA & Plasma & No \\
MP15 & V & 0.02 & $7 \mathrm{~d}$ & SVA & Plasma & Incomplete \\
MP16 & IX & 0.04 & $3 \mathrm{~d}$ & SVA & Plasma & yes \\
MP17 & V & 0.04 & $3 \mathrm{~d}$ & SVA & Plasma & yes \\
\hline MP18 & XI & 0.02 & $1 \mathrm{~d}$ & SVA & Plasma & yes \\
MP19 & XI & 0.02 & $3 \mathrm{~d}$ & SVA & Plasma & yes \\
MP20 & XI & 0.04 & $1 \mathrm{~d}$ & SVA & Plasma & yes \\
MP21 & XI & 0.04 & 3d & SVA & Plasma & yes \\
\hline & & & & & & \\
\hline
\end{tabular}




\subsection{Functionalization of Surfaces}

\subsubsection{Immobilization of RAFT Agent onto a Mesoporous Silica Film}

The surface modification of the mesoporous silica films with RAFT agent $\mathrm{BT}^{3} \mathrm{PT}$ was accomplished by following the procedure reported by Huebner et al. with minor changes. ${ }^{244]}$ Plasma-cleaned mesoporous thin films were immersed in a solution constituted of BT ${ }^{3} \mathrm{PT}(0.03 \mathrm{mmol})$ and 1,2-dimethoxyethane $(3 \mathrm{~mL})$. The mixture was gently shaken for $1 \mathrm{~d}$ at room temperature. Then, the mesoporous thin films were washed three times with acetone and dried under a stream of nitrogen.

\subsection{Polymerization}

\subsubsection{Homopolymerization of Styrene with polyRAFT}

Styrene polymerizations were conducted in bulk. In a typical polymerization procedure a solution containing RAFT agent and AIBN in styrene was mixed in a vial. The polymerization vial was purged with dry argon for $15 \mathrm{~min}$ in order to remove oxygen from the solution. After degassing, the polymerization vial was transferred into a heating block at $60^{\circ} \mathrm{C}$. The polymerization was stopped by cooling the vial in an ice bath and exposure to air. The obtained polymer was dissolved in a small amount of THF, precipitated in methanol and collected by centrifugation. The precipitation-centrifugation process was repeated three times to remove residual monomer and initiator.

Table 6.2. Bulk polymerization of styrene with different polyfunctional RAFT agents.

\begin{tabular}{cccc}
\hline$T /{ }^{\circ} \mathbf{C}$ & polyRAFT & $c_{\text {RAFT }} / \mathbf{m m o l} \cdot \mathrm{L}^{-1}$ & $c_{\text {AIBN }} /{\mathbf{~ m m o l} \cdot \mathbf{L}^{-1}}^{-1}$ \\
\hline $\mathbf{6 0}$ & A & 29.7 & 14.9 \\
$\mathbf{9 0}$ & A & 29.7 & 14.9 \\
$\mathbf{1 3 0}$ & $\mathrm{A}$ & 29.7 & - \\
$\mathbf{1 3 0}$ & $\mathrm{A}$ & 14.5 & - \\
$\mathbf{1 3 0}$ & $\mathrm{B}$ & 14.5 & - \\
$\mathbf{1 3 0}$ & $\mathrm{C}$ & 14.5 & - \\
\hline
\end{tabular}


Table 6.3. Bulk polymerization of styrene for the preparation of multiblock copolymers to investigate their microphase separation behavior.

\begin{tabular}{|c|c|c|c|c|c|}
\hline $\begin{array}{c}\text { multiblock } \\
\text { polystyrene for } \\
\text { sample }\end{array}$ & $\begin{array}{l}\mathrm{T} / \\
{ }^{\circ} \mathrm{C}\end{array}$ & $\begin{array}{l}t / \\
\mathrm{h}\end{array}$ & polyRAFT & $\begin{array}{c}c_{\mathrm{RAFT}} / \\
\mathrm{mmol} \cdot \mathrm{L}^{-1}\end{array}$ & $\begin{array}{c}c_{\text {AIBN }} / \\
\mathrm{mmol} \cdot \mathrm{L}^{-1}\end{array}$ \\
\hline I-IV, VI-VIII & 60 & 24 & $\bar{B}$ & 11.0 & 5.5 \\
\hline $\mathbf{V}$ & 60 & 24 & B & 10.2 & 5.7 \\
\hline IX & 60 & 12 & B & 11.0 & 5.5 \\
\hline$X, X I$ & 130 & 18 & A & 14.6 & - \\
\hline
\end{tabular}

\subsubsection{Block Copolymerization of Styrene Homopolymer with $n$-Butyl Acrylate}

In a typical procedure, m-PS (macroCTA), AIBN, n-butyl acrylate and toluene were placed into vials. Oxygen was removed from the solution by purging with dry argon for $15 \mathrm{~min}$. After degassing, the polymerization vial was transferred to a heating block at $60^{\circ} \mathrm{C}$. The polymerization was then stopped by cooling the vial in an ice bath and exposure to air. The obtained polymer was dissolved in a small amount of THF, precipitated in methanol and collected by centrifugation. The precipitation-centrifugation process was repeated three times to remove residual monomer and initiator. The amounts of the used substances and polymerization are collated in the Table 6.4.

Table 6.4.Copolymerization 1 of BA with multiblock polystyrene in toluene. Samples I-IV were polymerized under the same conditions.

\begin{tabular}{ccccc}
\hline polymerization & $\begin{array}{c}\mathrm{T} / \\
{ }^{\circ} \mathrm{C}\end{array}$ & $\boldsymbol{c}_{\mathrm{BA}} / \mathbf{m o l} \cdot \mathrm{L}^{-\mathbf{1}}$ & $\begin{array}{c}\boldsymbol{c}_{\text {macroRAFT }} / \\
\mathbf{m m o l} \cdot \mathrm{L}^{-1}\end{array}$ & $\begin{array}{c}\boldsymbol{c}_{\mathrm{AIBN}} / \\
\mathbf{m m o l} \cdot \mathrm{L}^{-\mathbf{1}}\end{array}$ \\
\hline $\mathbf{1}$ & 60 & 3.5 & 1.2 & 1.2 \\
\hline
\end{tabular}

\subsubsection{Block Copolymerization of Styrene Homopolymer with $\mathrm{N}$-Isopropylacrylamide}

In a typical procedure, m-PS (macroCTA), AIBN, $N$-isopropylacrylamide and DMF were weighed into vials. Oxygen was removed from the solution by purging with dry argon for $15 \mathrm{~min}$. After degassing, the polymerization vial was transferred to a heating block at $60{ }^{\circ} \mathrm{C}$. The polymerization was stopped by cooling the vial in an ice bath and exposure to air. The obtained polymer was dissolved in a small amount of THF, precipitated in methanol and collected by centrifugation. The 
precipitation-centrifugation process was repeated three times to remove residual monomer and initiator. The amounts of the used substances and polymerization are collated in Table 6.5 and Table 6.6.

Table 6.5. Copolymerization 2 of NIPAM with multiblock polystyrene in DMF.

\begin{tabular}{ccccc}
\hline polymerization & $\begin{array}{c}\mathrm{T} / \\
{ }^{\circ} \mathrm{C}\end{array}$ & $\begin{array}{c}c_{\text {PNIPAM}} / \\
\mathrm{mol} \cdot \mathrm{L}^{-\mathbf{1}}\end{array}$ & $\begin{array}{c}c_{\text {macroRAFT }} / \\
\mathrm{mmol} \cdot \mathrm{L}^{-1}\end{array}$ & $\begin{array}{c}c_{\text {AIBN }} / \\
\mathbf{m m o l} \cdot \mathrm{L}^{-\mathbf{1}}\end{array}$ \\
\hline $\mathbf{2}$ & 70 & 6.9 & 2.3 & 2.3 \\
\hline
\end{tabular}

Table 6.6. Copolymerization of NIPAM with multiblock polystyrene (see Table 6.3) in DMF.

\begin{tabular}{cccccc}
\hline polymerization & $\mathbf{T} /{ }^{\circ} \mathbf{C}$ & $\boldsymbol{t} / \mathbf{h}$ & $\boldsymbol{c}_{\text {PNIPAM} / \mathbf{~ m o l \cdot L}} \mathbf{- 1}^{-1}$ & $\begin{array}{c}\boldsymbol{c}_{\text {macroRAFT }} / \\
\mathbf{m m o l} \cdot \mathbf{L}^{-\mathbf{1}}\end{array}$ & $\begin{array}{c}\boldsymbol{c}_{\text {AIBN }} / \\
\mathbf{m m o l} \cdot \mathbf{L}^{-\mathbf{1}}\end{array}$ \\
\hline V & 70 & 24 & 7.6 & 0.2 & 0.2 \\
VI & 70 & 2 & 6.6 & 2.2 & 2.2 \\
VII & 70 & 6 & 6.6 & 2.2 & 2.2 \\
VIII & 70 & 7 & 6.6 & 2.2 & 2.2 \\
IX & 70 & 30 & 2.7 & 2.5 & 2.5 \\
X & 70 & 24 & 3.3 & 4.0 & 4.0 \\
$\mathbf{X}$ & 70 & 48 & 3.3 & 4.0 & 4.0 \\
\hline
\end{tabular}

\subsubsection{Cleavage of RAFT Groups}

The RAFT groups were cleaved by an excess of radicals. Therefore, the multiblock copolymers $(10 \mathrm{mg})$ and AIBN $(10 \mathrm{mg})$ were dissolved in THF $(1 \mathrm{~mL})$. The reaction solution was heated up to $85^{\circ} \mathrm{C}$ for $5 \mathrm{~h}$. Afterwards the polymers were dried and directly analyzed via SEC without any further purification.

\subsubsection{Surface-initiated RAFT Polymerization of $\mathrm{N}$-Isopropylacrylamide}

A stock solution of NIPAM $(2.5 \mathrm{~g}, 22 \mathrm{mmol})$, the respective RAFT agent (0.053 mmol) and 2,2'-azobis(2-methylpropionitrile) $(1.7 \mathrm{mg}, 0.011 \mathrm{mmol})$ in 1,2-dimethoxyethane $(7.4 \mathrm{~mL})$ was prepared and degassed using three freepump-thaw cycles. Silica thin films were added under an inert atmosphere and covered with the stock solution. The samples were then conducted into a preheated oil bath of $60^{\circ} \mathrm{C}$ and left for $36 \mathrm{~h}$. The polymerization was stopped by cooling the samples to $0^{\circ} \mathrm{C}$ and exposure to air. Subsequently, the silica thin films were washed three times with acetone and dried under 
a nitrogen stream. The polymerization solution was precipitated into cold diethyl ether and the resulting polymer was collected by centrifugation. The polymer was redissolved in a small amount of acetone, precipitated again in diethyl ether and centrifuged. The process was repeated once. The isolated polymers were dried in a vacuum at $45^{\circ} \mathrm{C}$.

\subsection{Microphase Separation of Multiblock Copolymers}

\subsection{1 $m$-(PS-b-PBA)}

A solution of $m$-(PS- $b$-PBA) in propylene glycol methyl ether acetate $(2.5 \mathrm{wt} \%, 20 \mu \mathrm{l})$ was spin coated $(2100 \mathrm{rpm}, 60 \mathrm{~s})$ onto a plasma-cleaned silicon wafer $(1 \times 1 \mathrm{~cm}$, Plano). Microphase-separated structures were obtained by thermal annealing in a vacuum oven at $180^{\circ} \mathrm{C}$ for $3 \mathrm{~d}$.

\subsection{2 $m$-(PS-b-PNIPAM)}

A solution of $m$-(PS- $b$-PNIPAM) in THF $(2 \mathrm{wt} \%, 20 \mu \mathrm{L})$ was spin coated (3500 rpm, $15 \mathrm{~s})$ onto a plasma-cleaned silicon wafer $(1 \times 1 \mathrm{~cm}$, Plano). The substrate was placed inside a solvent chamber, which was first evacuated and then exposure to the solvent vapor for $2 \mathrm{~h}$. The solvent annealing process was stopped by fast quenching which was executed by evacuating the solvent chamber to remove all solvent from the chamber. ${ }^{[99]}$ 


\section{Appendices}

\section{Appendix A}

\section{m-(PS-b-PBA)}
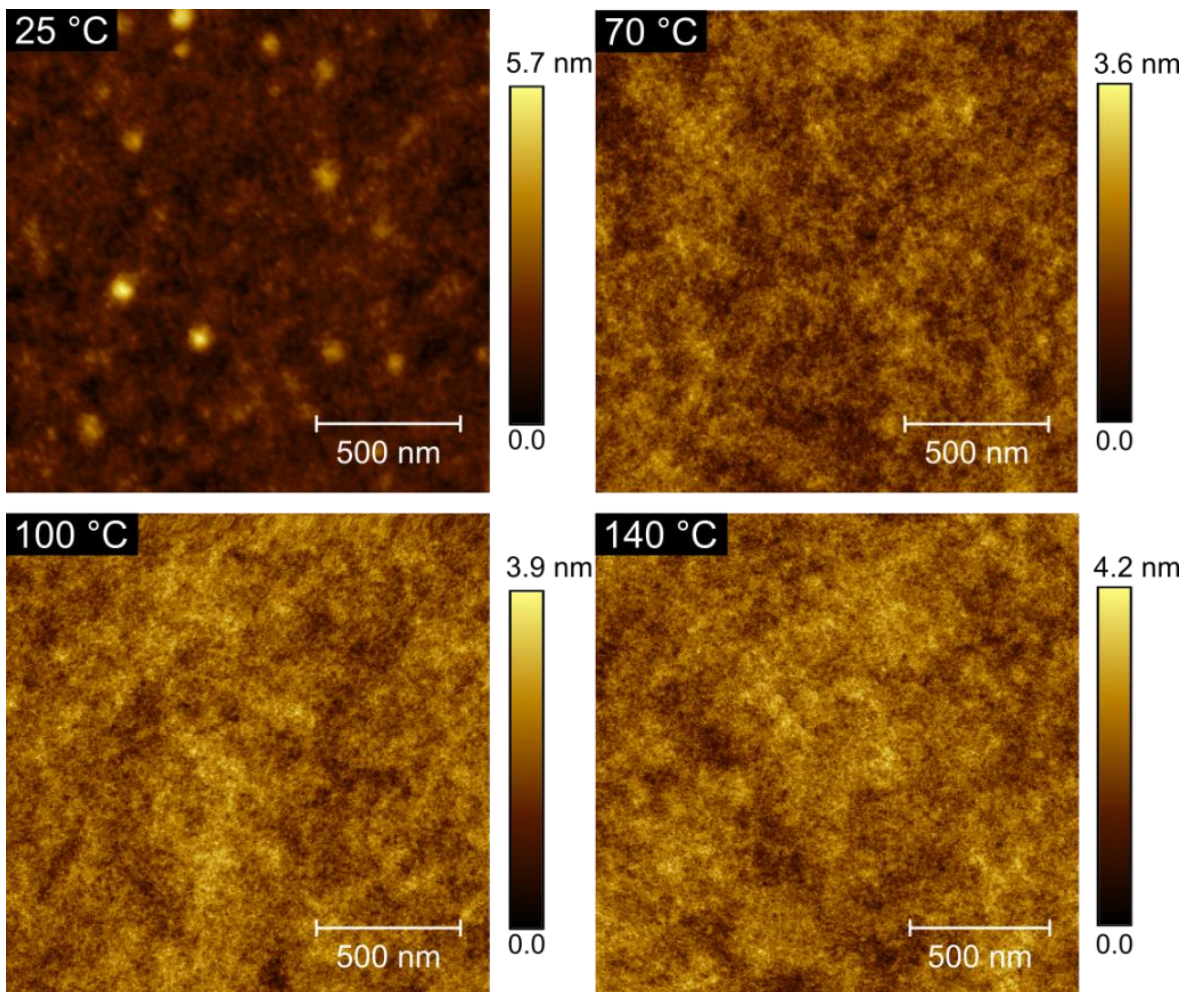

\section{$140{ }^{\circ} \mathrm{C}$}

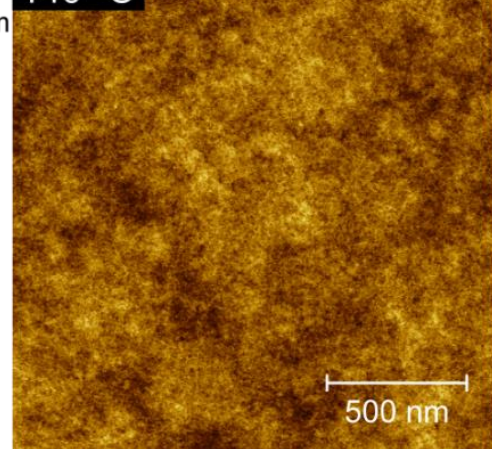

$4.2 \mathrm{~nm}$

Figure 6.1. AFM topography images of sample III at different annealing temperatures. 

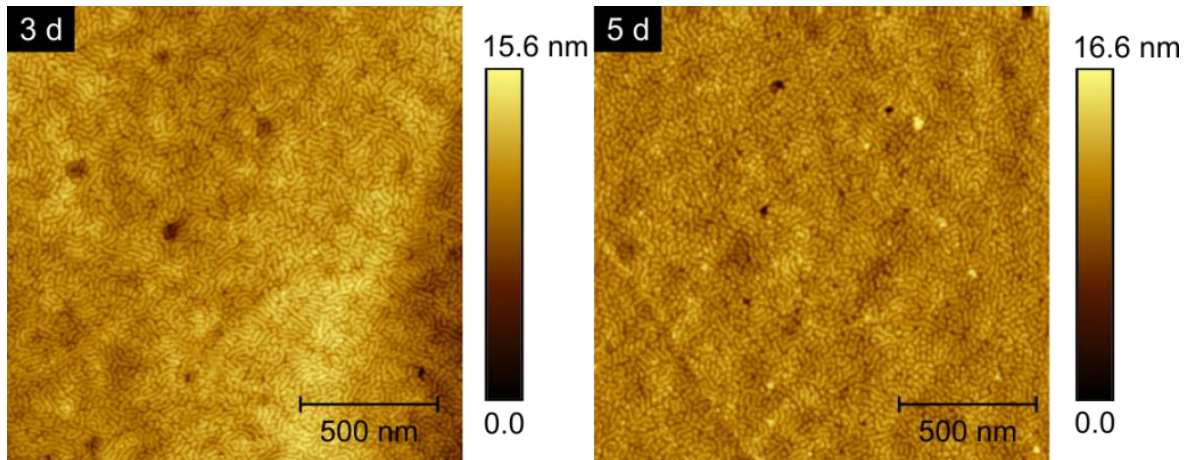

Figure 6.2. AFM topography images of sample III after thermal annealing for $3 \mathrm{~d}$ (left) and $5 \mathrm{~d}$ (right).

\section{$m$-(PS- $b$-PNIPAM)}
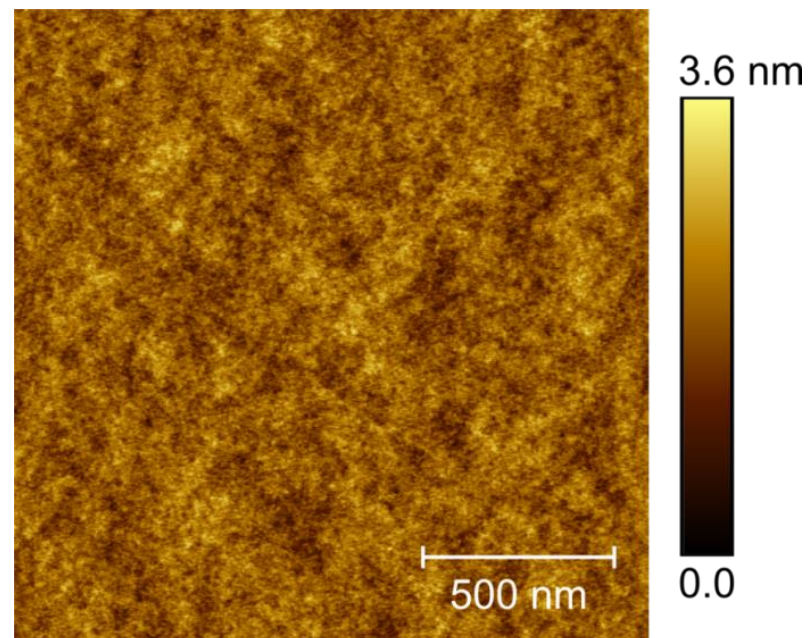

Figure 6.3. AFM topography image of sample $\mathbf{V}$ after thermal annealing for $3 \mathrm{~d}$ at $180^{\circ} \mathrm{C}$. 


\section{Appendix B}

\section{Mesoporous silica thin films via MBC}

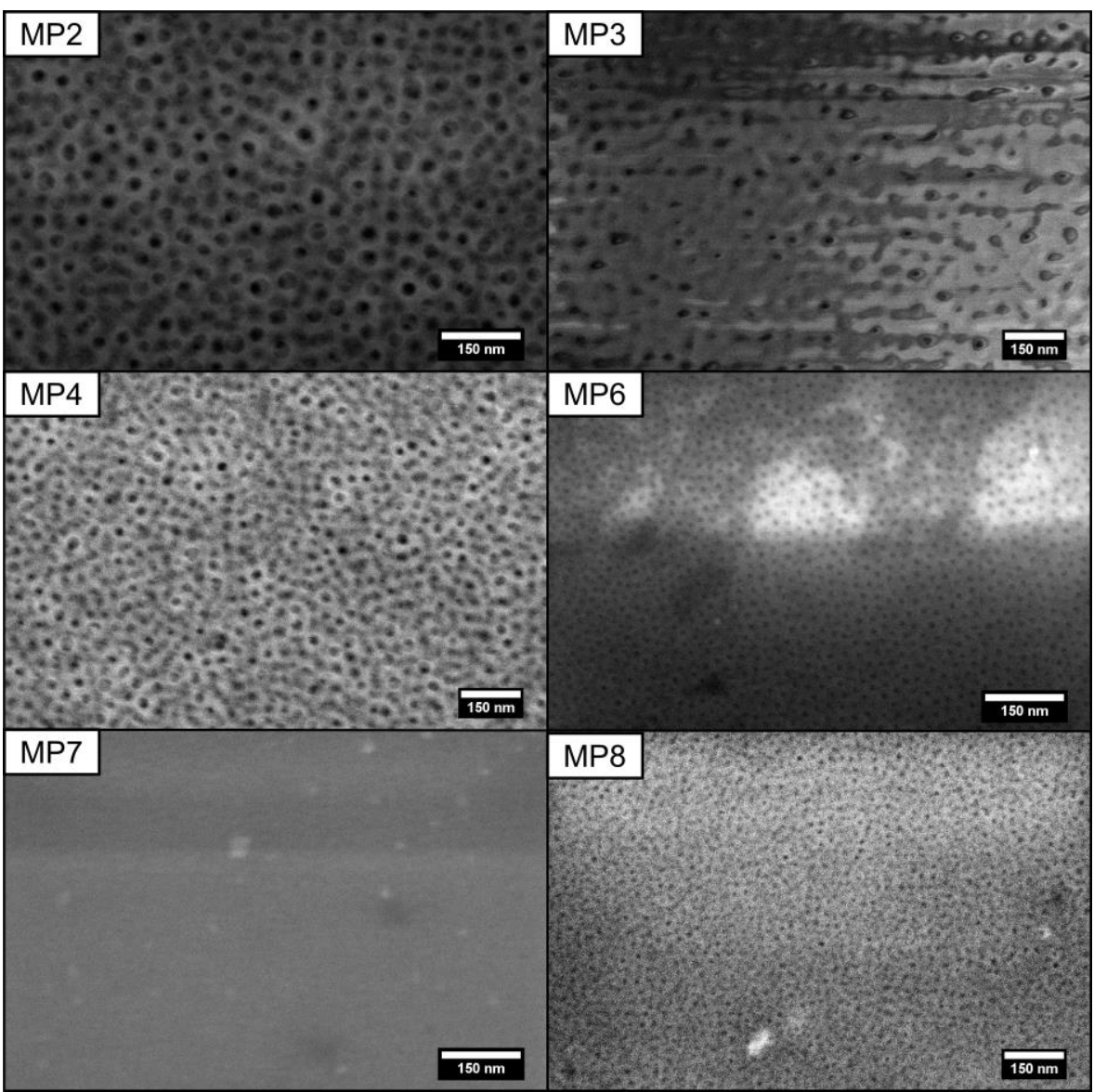

Figure 6.4. SEM images of mesoporous silica thin films using multiblock copolymers as organic template. 


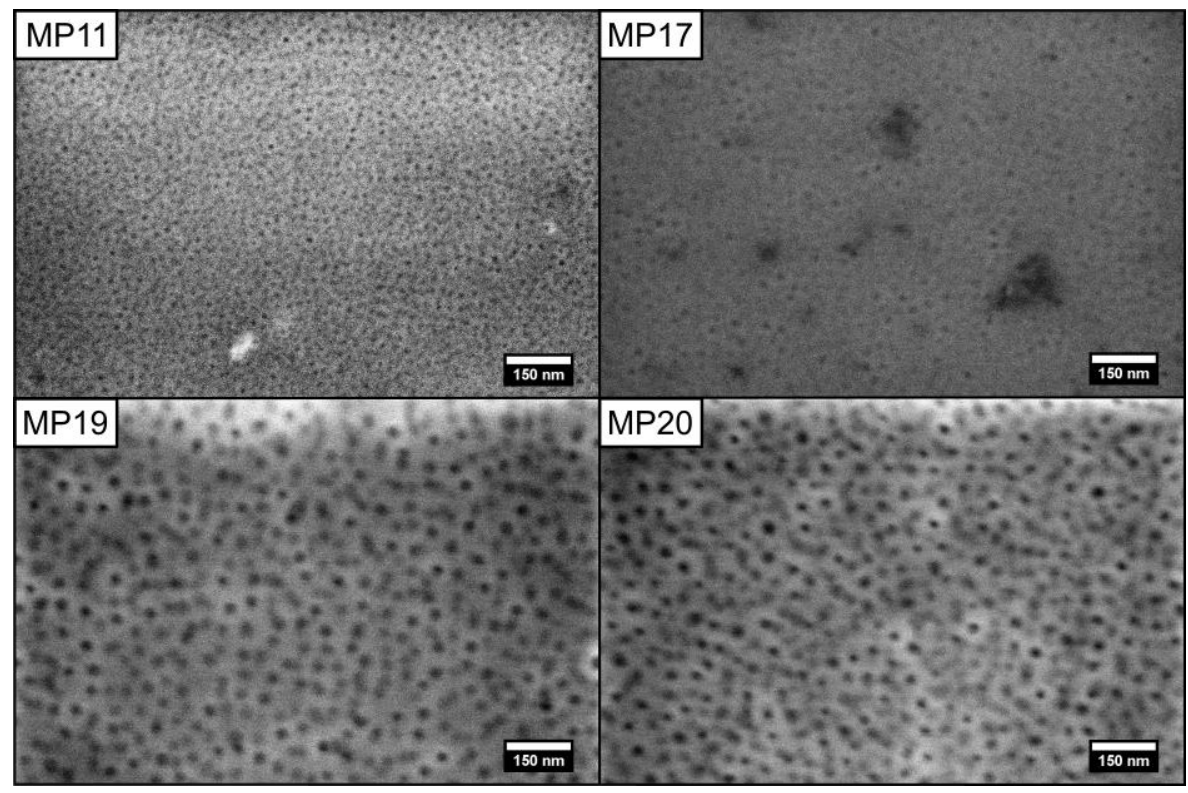

Figure 6.5. SEM images of mesoporous silica thin films using multiblock copolymers as organic template.

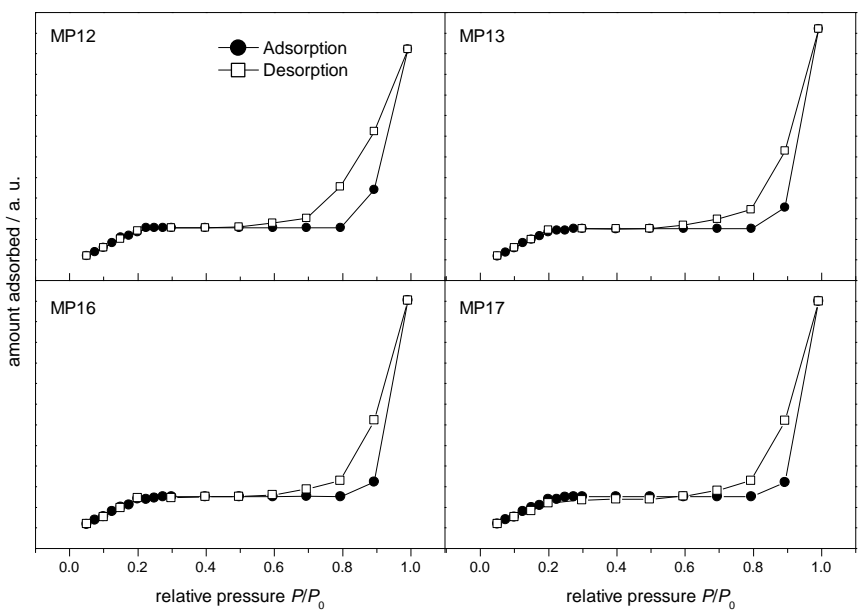

Figure 6.6. Nitrogen adsorption-desorption isotherms type IV with hysteresis loop H3. 


\section{Abbreviations}

\begin{tabular}{|c|c|}
\hline$\AA$ & Ångström $\left(10^{-10} \mathrm{~m}\right)$ \\
\hline AIBN & 2,2'-azobis(2-methylpropionitril) \\
\hline AFM & atomic force microscopy \\
\hline ATRP & atom-Transfer radical polymerization \\
\hline$b$ & block number \\
\hline BA & n-butyl acrylate \\
\hline BEMA & Brüggemann effective medium approximation \\
\hline $\mathrm{BT}^{3} \mathrm{PT}$ & benzyl(3-(trimethoxysilyl)propyl)trithiocarbonate \\
\hline${ }^{\circ} \mathrm{C}$ & degree Celsius \\
\hline$c$ & concentration \\
\hline $\mathrm{CD}_{2} \mathrm{Cl}_{2}$ & deuterated dichloromethane \\
\hline $\mathrm{CDCl}_{3}$ & deuterated chloroform \\
\hline $\mathrm{CV}$ & cyclic voltammetry \\
\hline 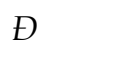 & dispersity $=\frac{\bar{M}_{\mathrm{w}}}{\bar{M}_{\mathrm{n}}}$ \\
\hline DSC & differential scanning calorimetry \\
\hline DLS & dynamic light scattering \\
\hline DMAc & $N, N$-dimethylacetamide \\
\hline $\mathrm{DMF}$ & $N, N$-dimethylformamide \\
\hline EEP & environmental ellipsometric porosimetry \\
\hline EISA & evaporation-induced self-assembly \\
\hline eq. & equivalent \\
\hline et al. & et alli \\
\hline$f_{\mathrm{A}}$ & volume fraction of monomer $\mathrm{A}$ \\
\hline$\phi_{\mathrm{A}}$ & comonomer composition of monomer A \\
\hline $\mathrm{g}$ & gram \\
\hline $\mathrm{h}$ & hours \\
\hline$I$ & integral \\
\hline ITO & indium tin oxide \\
\hline IUPAC & International Union of Pure and Applied Chemistry \\
\hline$r$ & surface energy \\
\hline K & Mark-Houwink-Parameter \\
\hline$k_{\mathrm{B}}$ & Boltzmann constant \\
\hline$l^{\prime}$ & Kuhn length \\
\hline
\end{tabular}




$\begin{array}{ll}L & \text { contour length } \\ \text { LCST } & \text { lower critical solution temperature } \\ \lambda & \text { wavelength } \\ m & \text { multiblock } \\ \bar{M}_{\mathrm{n}} & \text { number average molar mass } \\ \text { macroCTA } & \text { macromolecular controlling agent } \\ \text { MBC } & \text { multiblock copolymer } \\ \text { MS } & \text { mass spectroscopy } \\ \text { MeOH } & \text { methanol } \\ \text { min } & \text { minutes } \\ \text { MTF } & \text { mesoporous silica thin films } \\ \text { MSS } & \text { modulable steady state } \\ N & \text { degree of polymerization } \\ N \text { TTC } & \text { number of trithiocarbonate groups } \\ \text { NIPAM } & \text { N-isopropylacrylamide } \\ \text { NMP } & \text { nitroxide-mediated polymerization } \\ \text { NMR } & \text { nuclear magnetic resonance } \\ \text { ODT } & \text { order-disorder transition } \\ p & \text { porosity } \\ \text { PBA } & \text { poly(n-butyl acrylate) } \\ \text { PGMEA } & \text { propylene glycol methyl ether acetate } \\ \text { PNIPAM } & \text { poly(N-isopropylacrylamide) } \\ \text { polyRAFT } & \text { polyfunctional RAFT agent } \\ \text { ppm } & \text { parts per million } \\ \text { PSD } & \text { power spectral density function } \\ \text { PS } & \text { poly(styrene) } \\ \text { R } & \text { ideal gas constant } \\ \text { RAFT } & \text { reversible addition-fragmentation chain transfer } \\ \text { RDRP } & \text { reversible deactivation radical polymerization } \\ \text { RI } & \text { refractive index } \\ S & \text { segment number } \\ \delta & \text { solubility parameter } \\ \text { SAXS } & \text { small angle X-ray scattering } \\ \text { SEC } & \text { size-exclusion-chromatography } \\ \text { SEM } & \text { scanning electron microscopy } \\ \text { SVA } & \text { solvent (vapor) annealing } \\ T & \text { temperature } \\ T_{\mathrm{g}} & \text { glass transition temperature } \\ \text { TGA } & \text { thermogravimetric analysis } \\ \text { THF } & \text { tetrahydrofuran } \\ \text { Tol } & \text { toluene } \\ \text { TRP } & \text { Transient Receptor Potential } \\ & \end{array}$




$\begin{array}{ll}\text { TTC } & \text { trithiocarbonate } \\ \text { TTS } & \text { tunable steady state } \\ \text { UV } & \text { ultra violet } \\ \text { vis } & \text { visible } \\ \text { wt } \% & \text { weight percent } \\ X & \text { Flory-Huggins-interaction parameter } \\ \text { XPS } & \text { X-ray photoelectron spectroscopy }\end{array}$




\section{Bibliography}

[1] J. Livage, New J. Chem. 2001, 25, 1-1.

[2] A. E. Danks, S. R. Hall, Z. Schnepp, Mater. Horizons 2016, 3, 91-112.

[3] S. Kulprathipanja, Ed. , Zeolites in Industrial Separation and Catalysis, Wiley-VCH Verlag GmbH \& Co. KGaA, Weinheim, Germany, 2010.

[4] S. Förster, T. Plantenberg, Angew. Chem. Int. Ed. 2002.

[5] C. T. Kresge, M. E. Leonowicz, W. J. Roth, J. C. Vartuli, J. S. Beck, Nature 1992, 359, 710-712.

[6] J. S. Beck, J. C. Vartuli, W. J. Roth, M. E. Leonowicz, C. T. Kresge, K. D. Schmitt, C. T. W. Chu, D. H. Olson, E. W. Sheppard, S. B. McCullen, et al., J. Am. Chem. Soc. 1992, 114, 10834-10843.

[7] I. Ichinose, T. Kunitake, Chem. Rec. 2002, 2, 339-351.

[8] G. J. A. A. Soler-Illia, P. C. Angelomé, M. C. Fuertes, A. Calvo, A. Wolosiuk, A. Zelcer, M. G. Bellino, E. D. Martínez, J. Sol-Gel Sci. Technol. 2011, 57, 299-312.

[9] T. J, W. Y, E. W. McFarlandc, G. D. Stucky, Chem. Commun. 2004, 14, 1670-1671.

[10] L. Kavan, J. Rathouský, M. Grätzel, V. Shklover, A. Zukal, Microporous Mesoporous Mater. 2001, 44-45, 653-659.

[11] G. Wirnsberger, B. J. Scott, G. D. Stucky, Chem. Commun. 2001, 119120.

[12] M. D. Pérez, E. Otal, S. A. Bilmes, G. J. A. A. Soler-Illia, E. L. Crepaldi, D. Grosso, C. Sanchez, Langmuir 2004, 20, 6879-6886.

[13] T. A. García, C. A. Gervasi, M. J. Rodríguez Presa, J. I. Otamendi, S. E. Moya, O. Azzaroni, J. Phys. Chem. C 2012, 116, 13944-13953.

[14] S. Alberti, G. J. A. A. Soler-Illia, O. Azzaroni, Chem. Commun. 2015, 51, 6050-6075.

[15] B. Ebeling, P. Vana, Polymers 2011, 3, 719-739.

[16] C. Park, J. Yoon, E. L. Thomas, Polymer 2003, 44, 6725-6760.

[17] R. E. Richard, M. Schwarz, S. Ranade, A. K. Chan, K. Matyjaszewski, B. Sumerlin, Biomacromolecules 2005, 6, 3410-3418.

[18] P. Escalé, M. Save, A. Lapp, L. Rubatat, L. Billon, Soft Matter 2010, 6, 3202-3210.

[19] S. Ji, L. Wan, C. C. Liu, P. F. Nealey, Prog. Polym. Sci. 2016, 54-55, 76127. 
[20] C. Rossner, B. Ebeling, P. Vana, ACS Macro Lett. 2013, 2, 1073-1076.

[21] J. Zhang, R. Deubler, M. Hartlieb, L. Martin, J. Tanaka, E. Patyukova, P. D. Topham, F. H. Schacher, S. Perrier, Macromolecules 2017, 50, 7380-7387.

[22] M. T. Martello, D. K. Schneiderman, M. A. Hillmyer, ACS Sustain. Chem. Eng. 2014, 2, 2519-2526.

[23] T. R. Panthani, F. S. Bates, Macromolecules 2015, 48, 4529-4540.

[24] I. Lee, F. S. Bates, Macromolecules 2013, 46, 4529-4539.

[25] R. J. Spontak, S. D. Smith, J. Polym. Sci. Part B Polym. Phys. 2001, 39, 947-955.

[26] H. Benoit, G. Hadziioannou, Macromolecules 1988, 21, 1449-1464.

[27] T. A. Kavassalis, M. D. Whitmore, Macromolecules 1991, 24, 53405345.

[28] J. M. Zielinski, R. J. Spontak, Macromolecules 1992, 25, 653-662.

[29] M. W. Matsen, M. Schick, Macromolecules 1994, 27, 7157-7163.

[30] L. Wu, E. W. Cochran, T. P. Lodge, F. S. Bates, Macromolecules 2004, 37, 3360-3368.

[31] K. O. Rasmussen, E. M. Kober, T. Lookman, A. Saxena, J. Polym. Sci. Part B Polym. Phys. 2002, 41, 104-111.

[32] G. Gody, T. Maschmeyer, P. B. Zetterlund, S. Perrier, Macromolecules 2014, 47, 3451-3460.

[33] G. Gody, R. Barbey, M. Danial, S. Perrier, Polym. Chem. 2015, 6, 15021511.

[34] N. a. Hadjiantoniou, T. Krasia-Christoforou, E. Loizou, L. Porcar, C. S. Patrickios, Macromolecules 2010, 43, 2713-2720.

[35] S. Motokucho, A. Sudo, F. Sanda, T. Endo, Chem. Commun. 2002, 1946-1947.

[36] Y.-Z. You, C.-Y. Hong, C.-Y. Pan, Chem. Commun. 2002, 2800-2801.

[37] Y. You, C. Hong, C. Pan, Macromol. Rapid Commun. 2002, 23, 776780.

[38] B. Ebeling, M. Eggers, P. Vana, Macromolecules 2010, 43, 10283-10290.

[39] J. Chiefari, Y. K. B. Chong, F. Ercole, J. Krstina, J. Jeffery, T. P. T. Le, R. T. A. Mayadunne, G. F. Meijs, C. L. Moad, G. Moad, et al., Macromolecules 1998, 31, 5559-5562.

[40] G. Moad, A. E. Rizzardo, S. H. T. A, Aust. J. Chem. 2006, 59, 669-692.

[41] G. Moad, B. E. Rizzardo, S. H. T. A, Aust. J. Chem. 2009, 62, 14021472.

[42] G. Moad, E. Rizzardo, S. H. Thang, Aust. J. Chem. 2012, 65, 985-1076.

[43] M. Destarac, C. Brochon, J.-M. Catala, A. Wilczewska, S. Z. Zard, Macromol. Chem. Phys. 2002, 203, 2281-2289.

[44] M. Destarac, W. Bzducha, D. Taton, I. Gauthier-Gillaizeau, S. Z. Zard, Macromol. Rapid Commun. 2002, 23, 1049-1054. 
[45] M. A. Harvison, A. B. Lowe, Macromol. Rapid Commun. 2011, 32, 779800.

[46] M. N. Teerenstra, D. R. Suwier, B. Van Mele, L. Teuwen, M. Maassen, H. J. den Berg, C. E. Koning, J. Polym. Sci., Part A Polym. Chem. 2000, 38, 3550-3557.

[47] T. Yamaoka, Y. Takahashi, T. Ohta, M. Miyamoto, A. Murakami, Y. Kimura, J. Polym. Sci., Part A: Polym. Chem. 1999, 37, 1513-1521.

[48] F. Samperi, R. Mendichi, L. Sartore, M. Penco, C. Puglisi, Macromolecules 2006, 39, 9223-9233.

[49] D. Husken, J. Feijen, R. J. Gaymans, J. Polym. Sci., Part A: Polym. Chem. 2007, 45, 4522-4535.

[50] L. Song, B. Du, L. Chen, M. Deng, H. Sun, X. Pang, P. Zhang, X. Chen, J. Polym. Sci., Part A: Polym. Chem. 2013, 51, 4722-4731.

[51] M. Jikei, Y. Takeyama, Y. Yamadoi, N. Shinbo, K. Matsumoto, M. Motokawa, K. Ishibashi, F. Yamamoto, Polym. J. 2015, 47, 657-665.

[52] G. Wang, M. Jiang, Q. Zhang, R. Wang, G. Zhou, Polym. Degrad. Stab. 2017, 144, 121-127.

[53] X. Zeng, B. Wu, L. Wu, J. Hu, Z. Bu, B. Li, Ind. Eng. Chem. Res. 2014, $53,3550-3558$.

[54] S. Arimori, S. Ohashi, T. Matsuda, React. Funct. Polym. 2007, 67, 1346-1360.

[55] C. Wu, Z. Xie, G. Zhang, G. Zi, Y. Tu, Y. Yang, P. Cai, T. Nie, Chem. Commun. 2002, 0, 2898-2899.

[56] Y. Matsushita, Y. Mogi, H. Mukai, J. Watanabe, I. Noda, Polymer 1994, 35, 246-249.

[57] G. Gody, T. Maschmeyer, P. B. Zetterlund, S. Perrier, Nat. Commun. 2013, 4, 2505.

[58] G. Gody, T. Maschmeyer, P. B. Zetterlund, S. Perrier, Macromolecules 2014, 47, 639-649.

[59] B. Ebeling, P. Vana, Macromolecules 2013, 46, 4862-4871.

[60] M. Hendrich, L. Lewerdomski, P. Vana, J. Polym. Sci., Part A: Polym. Chem. 2015, 53, 2809-2819.

[61] Y. Liu, K. a. Cavicchi, Macromol. Chem. Phys. 2009, 210, 1647-1653.

[62] P. Lei, Q. Wang, J. Hong, Y. Li, J. Polym. Sci., Part A: Polym. Chem. 2006, 44, 6600-6606.

[63] J. Hong, Q. Wang, Y. Lin, Z. Fan, Macromolecules 2005, 38, 2691-2695.

[64] J. Hong, Q. Wang, Z. Fan, Macromol. Rapid Commun. 2006, 27, 57-62.

[65] L. Zhang, Q. Wang, P. Lei, X. Wang, C. Wang, L. Cai, J. Polym. Sci., Part A: Polym. Chem. 2007, 45, 2617-2623.

[66] Y. Wu, Q. Wang, J. Polym. Sci., Part A: Polym. Chem. 2010, 48, 24252429.

[67] R. Bussels, C. E. Koning, Tetrahedron 2005, 61, 1167-1174. 
[68] R. Bussels, C. Bergman-Göttgens, J. Meuldijk, C. Koning, Polymer 2005, 46, 8546-8554.

[69] R. Bussels, C. Bergman-Göttgens, J. Meuldijk, C. Koning, Macromolecules 2004, 37, 9299-9301.

[70] J. Jonikaitė-Švėgždienė, T. Krivorotova, T. Joskaudas, R. Makuška, Chemija 2015, 26, 60-68.

[71] Z. Jia, X. Xu, Q. Fu, J. Huang, J. Polym. Sci., Part A: Polym. Chem. 2006, 44, 6071-6082.

[72] Y. Zhou, K. Jiang, Q. Song, S. Liu, Langmuir 2007, 23, 13076-84.

[73] A.-L. Roy, C. Bui, I. Rau, F. Kajzar, B. Charleux, M. Save, D. Kreher, A.-J. Attias, Polymer 2014, 55, 782-787.

[74] J.-J. J. Yan, Z.-K. K. Wang, X.-S. S. Lin, C.-Y. Y. Hong, H.-J. J. Liang, C.-Y. Y. Pan, Y.-Z. Z. You, Adv. Mater. 2012, 24, 5617-5624.

[75] Q. Li, X. Hu, R. Bai, Macromol. Rapid Commun. 2015, 36, 1810-1815.

[76] M. Hendrich, P. Vana, Macromol. Mater. Eng. 2017, 302, 1700018.

[77] C. Rossner, O. Glatter, O. Saldanha, S. Köster, P. Vana, Langmuir 2015, 31, 10573-10582.

[78] C.-A. Fustin, A.-S. Duwez, J. Electron Spectros. Relat. Phenomena 2009, 172, 104-106.

[79] P. J. Flory, J. Am. Chem. Soc. 1936, 58, 1877-1885.

[80] F. S. Bates, G. H. Fredrickson, Phys. Today 1999, 52, 32.

[81] E. Helfand, Macromolecules 1975, 8, 552-556.

[82] E. Helfand, Z. R. Wasserman, Macromolecules 1976, 9, 879-888.

[83] E. Helfand, Z. R. Wasserman, Macromolecules 1978, 11, 960-966.

[84] E. Helfand, Z. Wasserman, Macromolecules 1980, 13, 994-998.

[85] L. Leibler, Macromolecules 1980, 13, 1602-1617.

[86] M. W. Matsen, F. S. Bates, Macromolecules 1996, 29, 1091-1098.

[87] M. W. Matsen, F. S. Bates, J. Chem. Phys. 1997, 106, 2436-2448.

[88] M. W. Matsen, M. Schick, Phys. Rev. Lett. 1994, 72, 2660-2663.

[89] D. J. Meier, J. Polym. Sci. Part C Polym. Symp. 1969, 26, 81-98.

[90] F. Bates, G. H. Fredrickson, Annu. Rev. Phys. Chem. 1990, 41, 525-557.

[91] Y. Mai, A. Eisenberg, Chem. Soc. Rev. 2012, 41, 5969.

[92] W. Zheng, Z.-G. Wang, Macromolecules 1995, 28, 7215-7223.

[93] V. Abetz, R. Stadler, Macromol. Symp. 1997, 113, 19-26.

[94] R. J. Spontak, S. D. Smith, A. Ashraf, Macromolecules 1993, 26, 51185124.

[95] M. W. Matsen, J. Chem. Phys. 1995, 102, 3884-3887.

[96] Q. Tang, J. Tang, M. Müller, Eur. Phys. J. Spec. Top. 2016, 225, 17851803.

[97] K. Tietz, S. Finkhäuser, K. Samwer, P. Vana, Macromol. Chem. Phys. 2014, 215, 1563-1572.

[98] R. A. Farrell, T. G. Fitzgerald, D. Borah, J. D. Holmes, M. A. Morris, 
Int. J. Mol. Sci. 2009, 10, 3671-3712.

[99] M. Cetintas, M. Kamperman, Polymer 2016, 107, 387-397.

[100] E. B. Gowd, M. Böhme, M. Stamm, IOP Conf. Ser. Mater. Sci. Eng. 2010, 14, 12015

[101] J. G. Son, K. W. Gotrik, C. A. Ross, ACS Macro Lett. 2012, 1, 12791284.

[102] K. Kim, S. Park, Y. Kim, J. Bang, C. Park, D. Y. Ryu, Macromolecules 2016, 49, 1722-1730.

[103] Z. Qiang, L. Zhang, G. E. Stein, K. A. Cavicchi, B. D. Vogt, Macromolecules 2014, 47, 1109-1116.

[104] W. I. Park, Y. J. Choi, J. M. Yun, S. W. Hong, Y. S. Jung, K. H. Kim, ACS Appl. Mater. Interfaces 2015, 7, 25843-25850.

[105] M. A. Chavis, D. M. Smilgies, U. B. Wiesner, C. K. Ober, Adv. Funct. Mater. 2015, 25, 3057-3065.

[106] S. S. Dinachali, W. Bai, K. Tu, H. K. Choi, J. Zhang, M. E. Kreider, L. Cheng, C. A. Ross, ACS Macro Lett. 2015, 4, 500-504.

[107] K. W. Gotrik, A. F. Hannon, J. G. Son, B. Keller, A. Alexander-Katz, C. A. Ross, ACS Nano 2012, 6, 8052-8059.

[108] J. K. Bosworth, M. Y. Paik, R. Ruiz, E. L. Schwartz, J. Q. Huang, A. W. Ko, D.-M. Smilgies, C. T. Black, C. K. Ober, ACS Nano 2008, 2, 1396-1402.

[109] K. Yamauchi, J. R. Lizotte, T. E. Long, Macromolecules 2003, 36, 10831088.

[110] G. J. A. A. Soler-Illia, O. Azzaroni, Chem. Soc. Rev. 2011, 40, 1107-50.

[111] A. M. Bivigou-Koumba, J. Kristen, A. Laschewsky, P. MüllerBuschbaum, C. M. Papadakis, Macromol. Chem. Phys. 2009, 210, 565578.

[112] J. Hentschel, A. M. Kushner, J. Ziller, Z. Guan, Angew. Chemie 2012, 124, 10713-10717.

[113] Y. Luo, X. Wang, Y. Zhu, B. G. Li, S. Zhu, Macromolecules 2010, 43, $7472-7481$.

[114] A. Nykänen, M. Nuopponen, A. Laukkanen, S.-P. Hirvonen, M. Rytelä, O. Turunen, H. Tenhu, R. Mezzenga, O. Ikkala, J. Ruokolainen, Macromolecules 2007, 40, 5827-5834.

[115] B. Y. K. Chong, T. P. T. Le, G. Moad, E. Rizzardo, S. H. Thang, Macromolecules 1999, 32, 2071-2074.

[116] R. T. A. Mayadunne, E. Rizzardo, J. Chiefari, J. Krstina, G. Moad, A. Postma, S. H. Thang, Macromolecules 2000, 33, 243-245.

[117] K. Skrabania, A. Miasnikova, A. M. Bivigou-Koumba, D. Zehm, A. Laschewsky, Polym. Chem. 2011, 2, 2074.

[118] G. Moad, E. Rizzardo, S. H. Thang, Polym. Int. 2011, 60, 9-25.

[119] W. Sriprom, M. James, S. Perrier, C. Neto, Macromolecules 2009, 42, 
3138-3146.

[120] T. Hashimoto, H. Tanaka, H. Hasegawa, Macromolecules 1990, 23, 4378-4386.

[121] T. G. Fox, P. J. Flory, J. Polym. Sci. 1954, 14, 315-319.

[122] T. G. Fox, P. J. Flory, J. Appl. Phys. 1950, 21, 581-591.

[123] H. A. Schneider, Polymer 2005, 46, 2230-2237.

[124] A. Rudin, D. Burgin, Polymer 1975, 16, 291-297.

[125] N. M. Ahmad, P. A. Lovell, S. M. Underwood, Polym. Int. 2001, 50, 625-634.

[126] A. Knoll, R. Magerle, G. Krausch, J. Chem. Phys. 2004, 120, 1105-1116.

[127] F. S. Bates, Science 1991, 251, 898-905.

[128] K. Tietz, Mikrophasenseparation von Photo-Vernetzbaren Blockcopolymeren in Dünnen Filmen, Georg-August-Universität Göttingen, 2014.

[129] S. B. Kaemmar, Bruker Appl. note AN133 2011, Rev. A0.

[130] B. Pittenger, N. Erina, C. Su, Bruker Appl. note AN 128 2012, Rev. B0.

[131] C. A. Rezende, L.-T. Lee, F. Galembeck, Langmuir 2009, 25, 9938 9946.

[132] E. A. G. J. Bandrup, E.H. Immergut, Ed. , Polymer Handbook, John Wiley \& Sons Inc, New York, 1999.

[133] Y. Yao, E. Metwalli, B. Su, V. Körstgens, D. Moseguí González, A. Miasnikova, A. Laschewsky, M. Opel, G. Santoro, S. V Roth, et al., ACS Appl. Mater. Interfaces 2015, 7, 13080-13091.

[134] V. S. Shinde, M. R. Girme, V. U. Pawar, Indian J. Chem. 2011, 50, 781787.

[135] B. Nandan, M. K. Vyas, M. Böhme, M. Stamm, Macromolecules 2010, $43,2463-2473$.

[136] C. Sinturel, M. Vayer, M. Morris, M. A. Hillmyer, Macromolecules 2013, 46, 5399-5415.

[137] S. O'Driscoll, G. Demirel, R. A. Farrell, T. G. Fitzgerald, C. O'Mahony, J. D. Holmes, M. A. Morris, Polym. Adv. Technol. 2011, 22, 915-923.

[138] A.-V. Ruzette, S. Tencé-Girault, L. Leibler, F. Chauvin, D. Bertin, O. Guerret, P. Gérard, Macromolecules 2006, 39, 5804-5814.

[139] P. J. Van Krevelen, D. W.; Hoftyzer, Properties of Polymers. Correlations with Chemical Structure, Elsevier, New York, 1972.

[140] D. R. Lide, Handbook of Organic Solvents, CRC Press, Boca Raton, FL, USA, 1995.

[141] M. Y. Paik, J. K. Bosworth, D.-M. Smilges, E. L. Schwartz, X. Andre, C. K. Ober, Macromolecules 2010, 43, 4253-4260.

[142] Y. Xuan, J. Peng, L. Cui, H. Wang, B. Li, Y. Han, Macromolecules 2004, 37, 7301-7307. 
[143] E. A. Jackson, Y. Lee, M. R. Radlauer, M. A. Hillmyer, ACS Appl. Mater. Interfaces 2015, 7, 27331-27339.

[144] U. Jeong, D. Y. Ryu, D. H. Kho, J. K. Kim, J. T. Goldbach, D. H. Kim, T. P. Russell, Adv. Mater. 2004, 16, 533-536.

[145] M. Shibayama, H. Hasegawa, T. Hashimoto, H. Kawai, Macromolecules 1982, 15, 274-280.

[146] C. T. Black, C. Forrey, K. G. Yager, Soft Matter 2017, 13, 3275-3283.

[147] K. A. Cavicchi, K. J. Berthiaume, T. P. Russell, Polymer 2005, 46, 11635-11639.

[148] I. W. Hamley, V. Castelletto, Prog. Polym. Sci. 2004, 29, 909-948.

[149] A. Tavakkoli K. G., S. M. Nicaise, K. R. Gadelrab, A. Alexander-Katz, C. A. Ross, K. K. Berggren, Nat. Commun. 2016, 7, 10518.

[150] M. Xing, W. Zhong, X. Xu, D. Thomson, Langmuir 2010, 26, 1180911814.

[151] V. P. Gilcreest, W. M. Carroll, Y. A. Rochev, I. Blute, K. A. Dawson, A. V. Gorelov, Langmuir 2004, 20, 10138-10145.

[152] H. Park, R. B. Thompson, N. Lanson, C. Tzoganakis, C. B. Park, P. Chen, J. Phys. Chem. B 2007, 111, 3859-3868.

[153] J. Zhang, R. Pelton, Colloids Surfaces A Physicochem. Eng. Asp. 1999, 156, 111-122.

[154] Z. Qian, V. S. Minnikanti, B. B. Sauer, G. T. Dee, W. G. Kampert, L. A. Archer, J. Polym. Sci. Part B Polym. Phys. 2009, 47, 1666-1685.

[155] G. J. A. A. Soler-Illia, C. Sanchez, B. Lebeau, J. Patarin, Chem. Rev. 2002, 102, 4093-4138.

[156] T. Yanagisawa, T. Shimizu, K. Kuroda, C. Kato, Bull. Chem. Soc. Jpn. 1990, 63, 1535-1537.

[157] A. Vinu, K. Z. Hossain, K. Ariga, J. Nanosci. Nanotechnol. 2005, 5, 347371.

[158] G. J. Galo, E. L. Crepaldi, D. Grosso, C. Sanchez, Curr. Opin. Colloid Interface Sci. 2003, 8, 109-126.

[159] E. L. Crepaldi, G. J. A. A. Soler-Illia, D. Grosso, F. Cagnol, F. Ribot, C. Sanchez, J. Am. Chem. Soc. 2003, 125, 9770-9786.

[160] M. K. Mayeda, J. Hayat, T. H. Epps, J. Lauterbach, J. Mater. Chem. A 2015, 3, 7822-7829.

[161] J. Rouquerol, D. Avnir, C. W. Fairbridge, D. H. Everett, J. M. Haynes, N. Pernicone, J. D. F. Ramsay, K. S. W. Sing, K. K. Unger, Pure Appl. Chem. 1994, 66, 1739-1758.

[162] J. R. and K. S. Françoise Rouquerol, in Adsorpt. by Powders Porous Solids, Elsevier, 1999.

[163] M. Thommes, K. Kaneko, A. V. Neimark, J. P. Olivier, F. RodriguezReinoso, J. Rouquerol, K. S. W. Sing, Pure Appl. Chem. 2015, 87, 10511069. 
[164] D. R. Ceratti, Viability of Nanoporous Films for Nanofluidic Applications, Université Pierre et Marie Curie, 2016.

[165] C. Brinker, G. Scherer, Sol-Gel Science: The Physics and Chemistry of Sol-Gel Processing, 1990.

[166] S. Polarz, M. Antonietti, Chem. Commun. 2002, 2593-2604.

[167] B. C. J. Brinker, Y. Lu, A. Sellinger, H. Fan, Adv. Mater. 1999, 11, 579585.

[168] H. Yang, A. Kuperman, N. Coombs, S. Mamiche-Afara, G. A. Ozin, Nature 1996, 379, 703-705.

[169] H. Yang, N. Coombs, I. Sokolov, G. A. Ozin, Nature 1996, 381, 589592.

[170] Y. Lu, H. Fan, A. Stump, T. L. Ward, T. Rieker, C. J. Brinker, Nature 1999, 398, 223-226.

[171] D. Grosso, F. Cagnol, G. J. A. A. Soler-Illia, E. L. Crepaldi, H. Amenitsch, A. Brunet-Bruneau, A. Bourgeois, C. Sanchez, Adv. Funct. Mater. 2004, 14, 309-322.

[172] L. Mahoney, R. Koodali, Materials 2014, 7, 2697-2746.

[173] D. Grosso, A. R. Balkenende, P. A. Albouy, A. Ayral, H. Amenitsch, F. Babonneau, Chem. Mater. 2001, 13, 1848-1856.

[174] I. Bischofberger, D. C. E. Calzolari, V. Trappe, Soft Matter 2014, 10, 8288-8295.

[175] D. R. Ceratti, M. Faustini, C. Sinturel, M. Vayer, V. Dahirel, M. Jardat, D. Grosso, Nanoscale 2015, 7, 5371-5382.

[176] K. Yu, A. J. Hurd, A. Eisenberg, C. J. Brinker, Langmuir 2001, 17, 7961-7965.

[177] A. Bouchara, G. Mosser, G. J. A. A. Soler-Illia, J.-Y. Chane-Ching, C. Sanchez, J. Mater. Chem. 2004, 14, 2347-2354.

[178] J. Polleux, M. Rasp, I. Louban, N. Plath, A. Feldhoff, J. P. Spatz, ACS Nano 2011, 5, 6355-6364.

[179] H.-G. Boyen, G. Kästle, K. Zürn, T. Herzog, F. Weigl, P. Ziemann, O. Mayer, C. Jerome, M. Möller, J. P. Spatz, et al., Adv. Funct. Mater. 2003, 13, 359-364.

[180] G. Kästle, H.-G. Boyen, F. Weigl, G. Lengl, T. Herzog, P. Ziemann, S. Riethmüller, O. Mayer, C. Hartmann, J. P. Spatz, et al., Adv. Funct. Mater. 2003, 13, 853-861.

[181] C. A. Schneider, W. S. Rasband, K. W. Eliceiri, Nat. Methods 2012, 9, 671-675.

[182] M. Haeri, M. Haeri, J. Open Res. Softw. 2015, 3, 2-5.

[183] X. Zhang, J. Wang, W. Wu, C. Liu, S. Qian, J. Sol-Gel Sci. Technol. 2007, 43, 305-311.

[184] D. Grosso, F. Babonneau, P. Albouy, H. Amenitsch, A. R. Balkenende, A. Brunet-Bruneau, J. Rivory, Chem. Mater. 2002, 14, 
931-939.

[185] K. S. W. Sing, D. H. Everett, R. A. W. Haul, L. Moscou, R. A. Pierotti, J. Rouquérol, T. Siemieniewska, Pure Appl. Chem. 1985, 57, 2201-2218.

[186] J. E. Spanier, I. P. Herman, Phys. Rev. B 2000, 61, 10437-10450.

[187] S. P. Naik, S. Yamakita, M. Ogura, T. Okubo, Microporous Mesoporous Mater. 2004, 75, 51-59.

[188] A. Halperin, Macromolecules 1991, 24, 1418-1419.

[189] B. Du, A. Mei, Y. Yang, Q. Zhang, Q. Wang, J. Xu, Z. Fan, Polymer 2010, 51, 3493-3502.

[190] C. Yu, J. Fan, B. Tian, G. D. Stucky, D. Zhao, J. Phys. Chem. B 2003, 107, 13368-13375.

[191] R. Lehner, X. Wang, M. Wolf, P. Hunziker, J. Control. Release 2012, 161, 307-316.

[192] B. V. V. S. P. Kumar, K. V. Rao, S. Sampath, S. J. George, M. Eswaramoorthy, Angew. Chemie Int. Ed. 2014, 53, 13073-13077.

[193] M. Ferrari, D. K. Martin, Nanobiotechnology of Biomimetic Membranes, Springer Science, New York, 2007.

[194] M. Tagliazucchi, Chemically Modified Nanopores and Nanochannels, Elsevier Inc., Oxford, 2016.

[195] Q. Ji, S. Acharya, J. P. Hill, A. Vinu, S. B. Yoon, J.-S. Yu, K. Sakamoto, K. Ariga, Adv. Funct. Mater. 2009, 19, 1792-1799.

[196] I. I. Slowing, B. G. Trewyn, V. S.-Y. Lin, The Supramolecular Chemistry of Organic-Inorganic Hybrid Materials, John Wiley \& Sons Inc, Hoboken, 2010.

[197] C. Sanchez, C. Boissiere, S. Cassaignon, C. Chaneac, O. Durupthy, M. Faustini, D. Grosso, C. Laberty-Robert, L. Nicole, D. Portehault, et al., Chem. Mater. 2014, 26, 221-238.

[198] P. Yang, S. Gai, J. Lin, Chem. Soc. Rev. 2012, 41, 3679.

[199] Q. He, J. Shi, J. Mater. Chem. 2011, 21, 5845.

[200] C. Coll, A. Bernardos, R. Martínez-Máñez, F. Sancenón, Acc. Chem. Res. 2013, 46, 339-349.

[201] D. Tarn, C. E. Ashley, M. Xue, E. C. Carnes, J. I. Zink, C. J. Brinker, Acc. Chem. Res. 2013, 46, 792-801.

[202] Z. Li, J. C. Barnes, A. Bosoy, J. F. Stoddart, J. I. Zink, Chem. Soc. Rev. 2012, 41, 2590.

[203] L. Minati, V. Antonini, M. Dalla Serra, G. Speranza, F. Enrichi, P. Riello, Microporous Mesoporous Mater. 2013, 180, 86-91.

[204] M. Yu, S. Jambhrunkar, P. Thorn, J. Chen, W. Gu, C. Yu, Nanoscale 2013, 5, 178-183.

[205] X. Wan, G. Zhang, S. Liu, Macromol. Rapid Commun. 2011, 32, 10821089.

[206] F. Tang, L. Li, D. Chen, Adv. Mater. 2012, 24, 1504-1534. 
[207] M. Hecht, E. Climent, M. Biyikal, F. Sancenón, R. Martínez-Máñez, K. Rurack, Coord. Chem. Rev. 2013, 257, 2589-2606.

[208] A. Brunsen, J. Cui, M. Ceolín, A. Del Campo, G. J. A. A. Soler-Illia, O. Azzaroni, Chem. Commun. 2012, 48, 1422-4.

[209] A. Calvo, B. Yameen, F. J. Williams, O. Azzaroni, G. J. A. A. SolerIllia, Chem. Commun. 2009, 2553-5.

[210] A. Brunsen, C. Díaz, L. I. Pietrasanta, B. Yameen, M. Ceolín, G. J. A. A. Soler-Illia, O. Azzaroni, Langmuir 2012, 28, 3583-3592.

[211] S. Angelos, E. Johansson, J. F. Stoddart, J. I. Zink, Adv. Funct. Mater. 2007, 17, 2261-2271.

[212] M. W. Ambrogio, C. R. Thomas, Y.-L. Zhao, J. I. Zink, J. F. Stoddart, Acc. Chem. Res. 2011, 44, 903-913.

[213] L. Du, S. Liao, H. A. Khatib, J. F. Stoddart, J. I. Zink, J. Am. Chem. Soc. 2009, 131, 15136-15142.

[214] X. Hou, H. Zhang, L. Jiang, Angew. Chemie Int. Ed. 2012, 51, 52965307.

[215] X. Hou, W. Guo, L. Jiang, Chem. Soc. Rev. 2011, 40, 2385.

[216] E. Aznar, C. Coll, M. D. Marcos, R. Martínez-Máñez, F. Sancenón, J. Soto, P. Amorós, J. Cano, E. Ruiz, Chem. - A Eur. J. 2009, 15, 68776888.

[217] A. Agostini, F. Sancenón, R. Martínez-Máñez, M. D. Marcos, J. Soto, P. Amorós, Chem. - A Eur. J. 2012, 18, 12218-12221.

[218] R. Mortera, J. Vivero-Escoto, I. I. Slowing, E. Garrone, B. Onida, V. S.-Y. Lin, Chem. Commun. 2009, 36, 3219.

[219] Y. Zhao, J. L. Vivero-Escoto, I. I. Slowing, B. G. Trewyn, V. S.-Y. Lin, Expert Opin. Drug Deliv. 2010, 7, 1013-1029.

[220] E. Climent, R. Martínez-Máñez, F. Sancenón, M. D. Marcos, J. Soto, A. Maquieira, P. Amorós, Angew. Chemie Int. Ed. 2010, 49, 7281-7283.

[221] E. Aznar, L. Mondragón, J. V. Ros-Lis, F. Sancenón, M. D. Marcos, R. Martínez-Máñez, J. Soto, E. Pérez-Payá, P. Amorós, Angew. Chemie Int. Ed. 2011, 50, 11172-11175.

[222] D. W. Urry, Angew. Chemie Int. Ed. 1993, 32, 819-841.

[223] H. Nakatsuji, T. Numata, N. Morone, S. Kaneko, Y. Mori, H. Imahori, T. Murakami, Angew. Chemie Int. Ed. 2015, 54, 11725-11729.

[224] B. Yameen, M. Ali, R. Neumann, W. Ensinger, W. Knoll, O. Azzaroni, Small 2009, 5, 1287-1291.

[225] W. Guo, H. Xia, F. Xia, X. Hou, L. Cao, L. Wang, J. Xue, G. Zhang, Y. Song, D. Zhu, et al., ChemPhysChem 2010, 11, 859-864.

[226] J. Huang, X. Zhang, P. A. McNaughton, Semin. Cell Dev. Biol. 2006, $17,638-645$.

[227] R. Latorre, S. Brauchi, G. Orta, C. Zaelzer, G. Vargas, Cell Calcium 2007, 42, 427-438. 
[228] H. Xu, I. S. Ramsey, S. A. Kotecha, M. M. Moran, J. A. Chong, D. Lawson, P. Ge, J. Lilly, I. Silos-Santiago, Y. Xie, et al., Nature 2002, 418, 181-186.

[229] M.-F. Tsai, C. Miller, Cell 2014, 158, 977-979.

[230] S. Chowdhury, B. W. Jarecki, B. Chanda, Cell 2014, 158, 1148-1158.

[231] K. Murakami, S. Watanabe, T. Kato, K. Sugawara, Colloids Surfaces A Physicochem. Eng. Asp. 2013, 419, 223-227.

[232] M. Bathfield, J. Reboul, T. Cacciaguerra, P. Lacroix-Desmazes, C. Gérardin, Chem. Mater. 2016, 28, 3374-3384.

[233] P.-W. Chung, R. Kumar, M. Pruski, V. S.-Y. Lin, Adv. Funct. Mater. 2008, 18, 1390-1398.

[234] A. de Sousa, D. A. Maria, R. G. de Sousa, E. M. B. de Sousa, J. Mater. Sci. 2010, 45, 1478-1486.

[235] J.-H. Park, Y.-H. Lee, S.-G. Oh, Macromol. Chem. Phys. 2007, 208, 2419-2427.

[236] M. M. Russell, L. Raboin, T. M. Guardado-Alvarez, J. I. Zink, J. SolGel Sci. Technol. 2014, 70, 278-285.

[237] H. G. Schild, Prog. Polym. Sci. 1992, 17, 163-249.

[238] A. Baeza, E. Guisasola, E. Ruiz-Hernández, M. Vallet-Regí, Chem. Mater. 2012, 24, 517-524.

[239] Q. Fu, G. V. R. Rao, L. K. Ista, Y. Wu, B. P. Andrzejewski, L. A. Sklar, T. L. Ward, G. P. López, Adv. Mater. 2003, 15, 1262-1266.

[240] Q. Fu, G. V Rama Rao, T. L. Ward, Y. Lu, G. P. Lopez, Langmuir 2007, 23, 170-174.

[241] Y. You, K. K. Kalebaila, S. L. Brock, D. Oupický, D. Qupický, Chem. Mater. 2008, 20, 3354-3359.

[242] M. D. Rowe-Konopacki, S. G. Boyes, Macromolecules 2007, 40, 879888.

[243] L. Lavanant, D. Paripovic, N. Schu, C. Sugnaux, S. Tugulu, H.-A. Klok, R. Barbey, L. Lavanant, D. Paripovic, N. Schüwer, et al., Chem. Rev. 2009, 109, 5437-527.

[244] D. Huebner, V. Koch, B. Ebeling, J. Mechau, J. E. Steinhoff, P. Vana, J. Polym. Sci., Part A: Polym. Chem. 2015, 53, 103-113.

[245] D. H. Nguyen, P. Vana, Polym. Adv. Technol. 2006, 17, 625-633.

[246] R. Rotzoll, D. H. Nguyen, P. Vana, Macromol. Symp. 2009, 275-276, 112.

[247] Y. Zhao, S. Perrier, Macromolecules 2006, 39, 8603-8608.

[248] V. G. Ngo, C. Bressy, C. Leroux, A. Margaillan, Polymer 2009, 50, 3095-3102.

[249] M. Semsarilar, S. Perrier, Nat. Chem. 2010, 2, 811-20.

[250] B. J. Lowes, A. G. Bohrer, T. Tran, D. A. Shipp, Polym. Bull. 2009, 62, 281-289. 
[251] M. Henze, D. Mädge, O. Prucker, J. Rühe, Macromolecules 2014, 47, 2929-2937.

[252] P. Brandani, P. Stroeve, Macromolecules 2004, 37, 6640-6643.

[253] S. Minko, S. Patil, V. Datsyuk, F. Simon, K. Eichhorn, M. Motornov, D. Usov, I. Tokarev, M. Stamm, Langmuir 2002, 18, 289-296.

[254] J. Elbert, F. Krohm, C. Rüttiger, S. Kienle, H. Didzoleit, B. N. Balzer, T. Hugel, B. Stühn, M. Gallei, A. Brunsen, Adv. Funct. Mater. 2014, 24, 1591-1601.

[255] B. Ebeling, F. Ehlers, P. Vana, Nachrichten aus der Chemie 2014, 62, 2428.

[256] M. Ma, S. Zheng, H. Chen, M. Yao, K. Zhang, X. Jia, J. Mou, H. Xu, R. Wu, J. Shi, J. Mater. Chem. B 2014, 2, 5828.

[257] G.-J. Wang, S.-Z. Huang, Y. Wang, L. Liu, J. Qiu, Y. Li, Polymer 2007, $48,728-733$.

[258] Y. Tsujii, M. Ejaz, K. Sato, A. Goto, T. Fukuda, Macromolecules 2001, 34, 8872-8878.

[259] A. Calvo, P. C. Angelomé, V. M. Sánchez, D. A. Scherlis, F. J. Williams, G. J. A. A. Soler-Illia, Chem. Mater. 2008, 20, 4661-4668.

[260] G. J. A. A. Soler-Illia, P. Innocenzi, Chem. - A Eur. J. 2006, 12, 44784494.

[261] Y. Zhao, S. Perrier, Macromolecules 2007, 40, 9116-9124.

[262] A. Chhabra, R. R. Kanapuram, T. J. Kim, J. Geng, A. K. da Silva, C. W. Bielawski, C. H. Hidrovo, Langmuir 2013, 130605123642004.

[263] C. Boissiere, D. Grosso, S. Lepoutre, L. Nicole, A. B. Bruneau, C. Sanchez, Langmuir 2005, 21, 12362-12371.

[264] M. Kruk, M. Jaroniec, Chem. Mater. 2003, 15, 2942-2949.

[265] S. Won, D. J. Phillips, M. Walker, M. I. Gibson, J. Mater. Chem. B 2016, 4, 5673-5682.

[266] J. Moraes, K. Ohno, T. Maschmeyer, S. Perrier, Chem. Commun. 2013, $49,9077$.

[267] A. Andrieu-Brunsen, S. Micoureau, M. Tagliazucchi, I. Szleifer, O. Azzaroni, G. J. A. A. Soler-Illia, Chem. Mater. 2015, 27, 808-821.

[268] M. Rafti, A. Brunsen, M. C. Fuertes, O. Azzaroni, G. J. A. A. SolerIllia, ACS Appl. Mater. Interfaces 2013, 5, 8833-8840.

[269] K. Ariga, M. Li, G. Richards, J. Hill, J. Nanosci. Nanotechnol. 2011, 11, 1-13.

[270] K. Ariga, Q. Ji, J. P. Hill, Y. Bando, M. Aono, NPG Asia Mater. 2012, 4, e17.

[271] K. Ariga, Q. Ji, W. Nakanishi, J. P. Hill, M. Aono, Mater, Horizons 2015, 2, 406-413.

[272] E. Aznar, R. Casasús, B. García-Acosta, M. D. Marcos, R. MartínezMáñez, F. Sancenón, J. Soto, P. Amorós, Adv. Mater. 2007, 19, 2228- 
2231.

[273] Y. Shiraishi, R. Miyamoto, T. Hirai, Org. Lett. 2009, 11, 1571-1574.

[274] G. N. Grover, J. Lee, N. M. Matsumoto, H. D. Maynard, Macromolecules 2012, 45, 4958-4965.

[275] S. Harrisson, K. L. Wooley, Chem. Commun. 2005, 3259.

[276] B. Tamami, A. R. Kiasat, J. Chem. Res. 1998, 454-455.

[277] P. C. Angelomé, G. J. A. A. Soler-Illia, J. Mater. Chem. 2005, 15, 3903. 
